Portland State University

PDXScholar

\title{
Simulation Design, Role Identification and Attitude Change in a High Technology Culture
}

Terry Robert Schumacher

Portland State University

Follow this and additional works at: https://pdxscholar.library.pdx.edu/open_access_etds Let us know how access to this document benefits you.

\section{Recommended Citation}

Schumacher, Terry Robert, "Simulation Design, Role Identification and Attitude Change in a High Technology Culture" (1992). Dissertations and Theses. Paper 1223.

https://doi.org/10.15760/etd.1222

This Dissertation is brought to you for free and open access. It has been accepted for inclusion in Dissertations and Theses by an authorized administrator of PDXScholar. Please contact us if we can make this document more accessible: pdxscholar@pdx.edu. 


\title{
SIMULATION DESIGN, ROLE IDENTIFICATION AND ATTITUDE CHANGE IN A HIGH TECHNOLOGY CULTURE
}

\author{
by \\ TERRY ROBERT SCHUMACHER
}

A dissertation submitted in partial fulfillment of the requirements for the degree of

\section{DOCTOR OF PHILOSOPHY \\ in \\ SYSTEMS SCIENCE}

Portland State University

(C) 1992 
TO THE OFFICE OF GRADUATE STUDIES:

The members of the Committee approve the dissertation of Terry Robert Schumacher presented June 11, 1992.

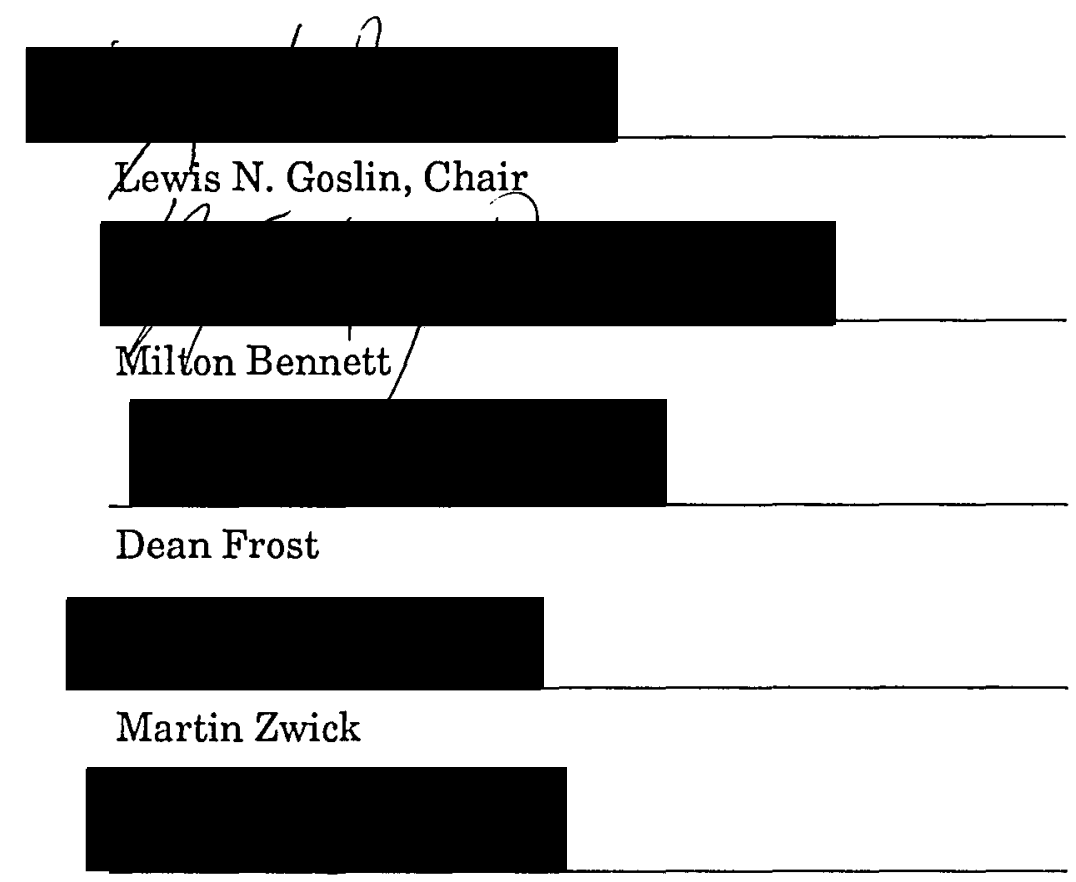

Sheldon Edner

APPROVED:

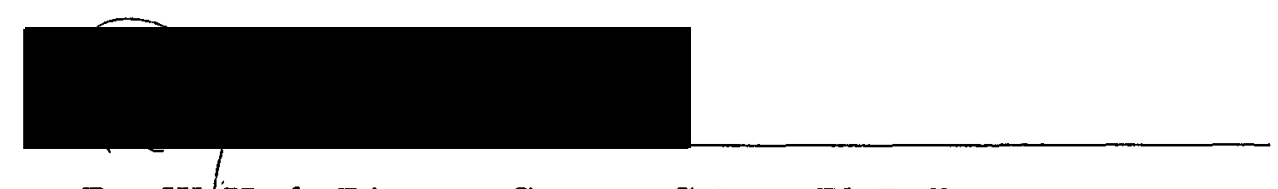

Roy W. Koch, Director, Systems Science Ph.D. Program

C. William Savery, Interim Vice Provost for Graduate Studies and Research 


\section{ACKNOWLEDGEMENTS}

The motivation to select corporate culture as a topic to be studied in this dissertation was born during seven years of employment as a strategic planner in the electric utility industry. It became clear to me that the decisions of an organization that considered itself "rational", were constrained and driven by the beliefs and values comprising the culture of that organization.

Looking at this literature I was disappointed to find few detailed empirical descriptions of organizational cultures. I believed that one thing this young field needed was an collection of ethnographies that sampled organizational cultures across industries, size, age and geography. Course work in intercultural communication had prepared me to do such an analysis. There I found a model for communication, Constructivism, which seemed to be an excellent theoretical perspective for approaching the analysis of culture. The dissertation I envisioned could provide one data point to begin to fill this need.

From the beginning this project was to go beyond qualitative description and address the question of culture change. The setting which had nurtured my interest in organizational culture had also provided strong motivation to consider directed change in the culture of an organization. The contact with simulation I received in my Systems Science course work and teaching, together with the theory and simulation experience in the intercultural communication courses, suggested to me that simulation could 
be one possible approach to culture change. This dissertation is my first attempt to explore simulation design effectiveness and changing organizational culture.

Certainly many people have made a contribution to this study. Hundreds of people at Mentor Graphics took time from their busy schedules to talk about their experiences in the Company. Many also filled out a long questionnaire, twice, so that I would have the quantitative data reported. More than one hundred also spent a day playing the WINNING AT DESIGN AUTOMATION simulation. Clearly this study would not have been possible without these contributions and I hope that those people feel that they benefit from this document and playing the simulation. I hope Mentor Graphics benefits from the use of the simulation and that they choose to maintain the 'small company atmosphere' for many years.

Dick Anderson, vice president of Human Resources at Mentor Graphics. Dick agreed to support the study after our initial meeting, waited patiently for results, and facilitated six of the eleven simulation sessions. Without this support the project could not have been completed. Peter Hoogerhuis offered me an experience of "Winning" at Mentor Graphics, an important contribution.

Dr. Steven Brenner certainly made a substantial contribution. During the early part of the work he demonstrated belief in the project and the student. That alone was very significant and a necessary condition for completion of this project. He pressured me to create a computer simulation when I favored a less technical format. Dr. Lew Goslin joined the committee late in the project and provided support and the guidance necessary to complete the writing of the thesis. I am grateful for his contribution. 
Harry Peterson-Nedry facilitated five of the eleven simulation sessions and expressed support for me and the simulation during the final phase of this project. That support was appreciated greatly. Bill Britton, in addition to acting as a technical reference as I learned HyperCard and ThunderScan, provided on-site technical support during the simulation sessions.

A number of people played the simulation during its development. Their feedback was extremely valuable in the evolution of the code and screen layouts. Dave Robison, Bob Block, Ron Reed, Peter Van Houton, Steve Brenner, Byrne Lovell, Don Wolfe, Barry Shane, Bill Britton, Wayne Wakeland, Sheldon Edner, Brian Willis, Dick Anderson, Harry PetersonNedry, and Mike Norman all made significant contributions as they struggled through bugs, crashes and the various interruptions that incomplete and partially tested computer code can generate.

I wish to extend a special thanks to Byrne Lovell who made many contributions throughout the study. He proof read the questionnaire and provided useful feedback. He endured late night discussions as the model of knowledge and frames of reference evolved. He not only played the simulation, but when my supply of virgin players was low he recruited others to play it. I hope my contributions to his dissertation are of as much value.

Finally I wish to dedicate this dissertation to my mother Patricia, who died while the manuscript was being completed, and to To-Chau, who came into my life during the project. 


\section{TABLE OF CONTENTS}

PAGE

ACKNOWLEDGEMENTS ............................................................................

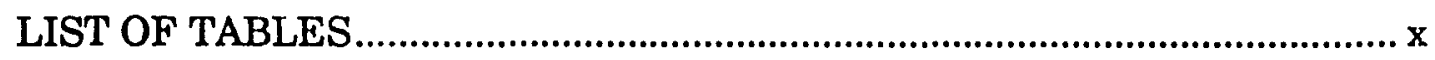

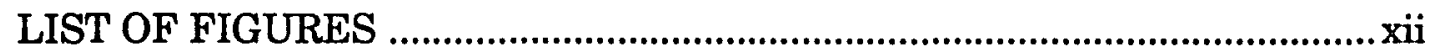

\section{CHAPTER}

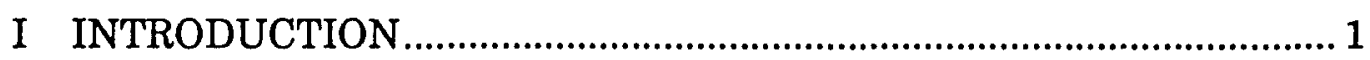

Simulation Design - Moving from Art toward Science.................... 1

Research Topics Addressed........................................................ 2

Evolution of the Causal Model.................................................... 4

Requirements and Structure of the Study ...................................... 6

Project Scope and Activities .....................................................

Summary of Findings ..................................................................... 9

Client Organization' - Mentor Graphics (MGC).......................... 11

II LITERATURE REVIEWED ............................................................ 13

Overview of Simulation and Attitude Change studies..................... 13

Methodological Concerns ........................................................ 14

The Need for Improved Theoretical Models.............................. 17

Simulation Structure Studies ........................................................... 19

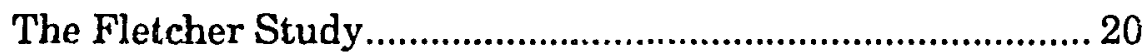

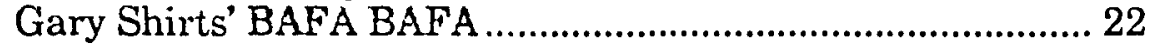

The Livingston and Kidder Study ............................................ 23

The Williams Studies......................................................... 26

Review of Simulation Structure Studies................................ 29

The Interpersonal Reactivity Index - Empathy ..................... 30 
Constructivist Models of Culture and Communication.

Overview of Constructivism .................................................. 32

Ethnoscience.................................................................... 34

Bennett's Empathy Model of Communication ...................... 36

Frame-of-Reference Switching, a "System-level" Model ........ 39

Summary of the Contribution of Constructivism ................... 42

Relevant Attitude Change Literature ........................................... 45

Historical Overview............................................................... 45

Attitude Definition ........................................................... 46

A Broader Context for Attitude Research ............................. 47

Schema Theories .................................................................. 49

Centrality of the Dependent Variables .................................. 52

The Measurement of Attitudes............................................... 53

III RESEARCH QUESTIONS AND HYPOTHESES ............................ 55

Definition of Variables ............................................................ 55

Statement of Research Questions.............................................. 57

Research Question 1 ....................................................... 57

Research Question 2 ......................................................... 58

Research Question 3 ...................................................... 58

Research Question 4 ..................................................... 59

Research Question 5 ...................................................... 59

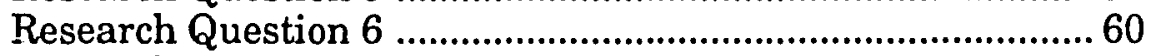

Research Question 7 ....................................................... 60

IV RESEARCH DESIGN AND METHODOLOGY..............................62

Research Design Overview......................................................6. 62

Qualitative Data Collection — Phase 1....................................... 63

Issue Selection Criteria..................................................... 63

Cover Story ..................................................................... 65

Primary Qualitative Data Source - Interviews .................... 65

Other Qualitative Data Sources ........................................... 69

Questionnaire Construction - Phase 2 ..................................... 71

Pilot Questionnaire and Reduction to Pre-Simulation

Questionnaire 
viii

PAGE

The "Quality Growth" Frame of Reference ...................................... 72

A New Frame of Reference - Quality Growth ....................... 74

Relationships among the Quality Growth Variables .............. 75

Measuring Movement Toward “Quality Growth" ...................... 77

Design of the Simulation Class - Phase 3 …………...................... 82

Simulation Class Features for the Alpha Game..................... 82

Class Features of the Beta Game............................................. 87

Class Features for the Other Treatment Groups .................... 87

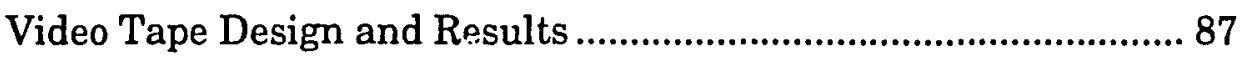

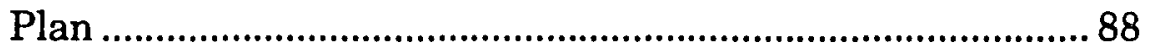

Execution .............................................................................. 88

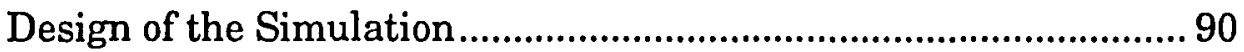

Computer Based .................................................................... 90

The Macro Level Structure ....................................................... 92

Role Identification Features of the WADA Simulation........... 93

Simulation Design Style ............................................................. 98

Suggesting a Preferred Cultural Role - Beta ........................ 99

Dynamics of Winning At Design Automation......................... 100

Experimental Procedures - Phase 4 ............................................... 101

Definition of Treatment Groups ............................................ 101

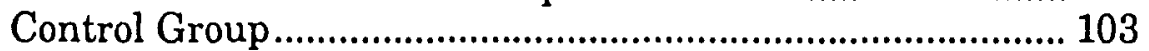

Assignment of Subjects to Treatment Groups ....................... 103

Simulation Class Sequence and Schedule .............................. 105

Facilitator Guidebook ........................................................ 108

Simulation Delivery ......................................................... 109

Post-Simulation Questionnaire ........................................... 110

Post-Simulation Interviews .................................................. 111

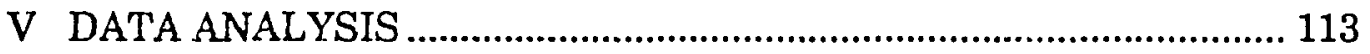

Questionnaire Structure And Reliability .......................................... 113

Expectations of Change for the Attitude Subscales .............. 115

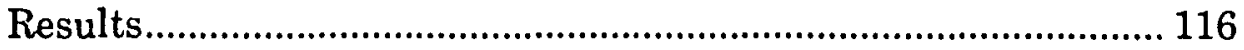

Research Question 1 ............................................................. 116

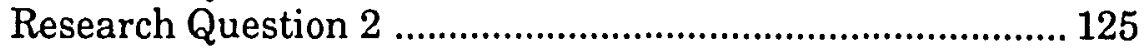


PAGE

Research Question 3 ........................................................ 129

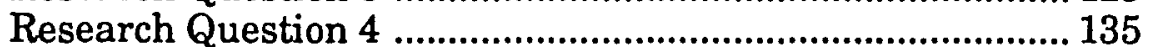

Research Question 5 ......................................................... 137

Research Question 6 .......................................................... 142

Research Question 7 ........................................................... 143

Other Questionnaire Items................................................ 151

VI SUMMARY AND DISCUSSION ................................................... 156

Summary ............................................................................ 156

Discussion of Results............................................................... 161

Primary Simulation Design Goal - Attitude Change ......... 161

Influence of Demographic Variables ................................... 164

Secondary Design Goal - Differential Role Identification ... 165

Role Identification and Attitude Change ............................. 166

Role-Culture Contrast and Attitude Change ....................... 167

Other Plausible Explanations for Observed Results............ 169

Qualitative Assessment of Simulation Effectiveness......... 173

Duration of Simulation Effect ............................................ 175

Cognitive Style ........................................................... 176

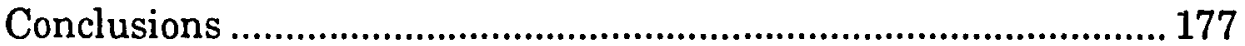

Suggestions for Future Research............................................ 181

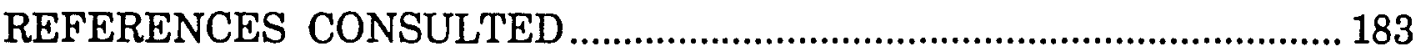

APPENDICES

A. CLIENT LETTER OF AGREEMENT ........................................... 193

B. MENTOR GRAPHICS CULTURAL TERMS.................................... 197

C. ETHNOGRAPHY OF MENTOR GRAPHICS ................................. 210

D. FINAL QUESTIONNAIRE ...................................................... 256

E. WINNING AT DESIGN AUTOMATION RULEBOOK..................... 267

F. FACILITATOR'S GUIDEBOOK .................................................. 283

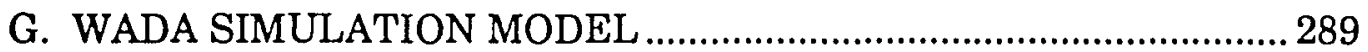




\section{LIST OF TABLES}

TABLE

PAGE

I Dependent Variable Correlation Matrix ...................................... 114

II Cronbach's Alpha for Dependent Variables.................................. 115

III Comparison of Sessions 1 and 2 ............................................ 118

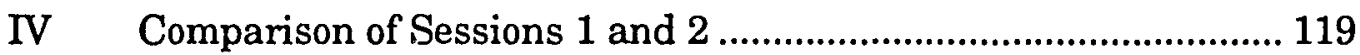

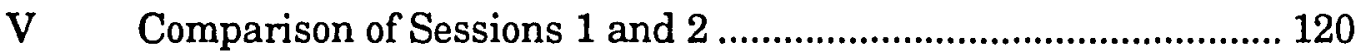

VI Anova Comparison of Attitude Change by Gender ...................... 120

VII Regression of Attitude Change on Age ........................................ 122

VIII Regression of Attitude Change on Employment Length............... 123

IX Control Group Scores (N=41) ..................................................... 126

X Simplified Simulation Group Scores (N=37) ............................... 127

XI Enhanced Simulation Group Scores $(\mathrm{N}=30)$.................................. 128

XII Video control Group Scores $(\mathrm{N}=28)$............................................ 128

XIII Mediating Variable Comparison .................................................... 133

XIV Mediating Variable Comparison ..................................................... 134

XV Perspective Taking Regression Models ........................................ 136

XVI Fantasy Regression Models ........................................................... 137

XVII Role Identification and Attitude Change ........................................ 139

XVIII Role Culture Contrast Regression Models ...................................... 140

XIX Role Culture Contrast and Attitude Change .................................. 141

XX Magnitude of Change Comparison (ES vs SS) ............................ 142

XXI Comparison of Capstone Variable Change ..................................... 144 
XXII Pre Simulation Dependent Variable Correlation Matrix.............. 146

XXIII Post Simulation Dependent Variable Correlation Matrix ........... 146

XXIV Average Dependent Variable Correlations ...................................... 147 


\section{LIST OF FIGURES}

$\begin{array}{lll}\text { FIGURE } & \text { PAGE }\end{array}$

1. Implicit causal model in previous simulation research....................... 4

2. Hypothesized causal model in the present study................................5

3. Diagram of research topic integration................................................... 7

4. Study phase, activities and outputs. .................................................... 9

5. Summary of Pierfy's methodological concerns.................................. 15

6. Comparison of causal models......................................................... 18

7. Thornton and Cleveland's simulation continuum............................ 20

8. Summary of suggested simulation attributes.................................... 29

9. Perspectives in social science, from Morgan and Smircich............. 33

10. Bennett's six-step model of empathy.................................................. 37

11. The concept of multiple stable frames of reference. .......................... 41

12. Mediating variables suggested by Constructivism............................ 44

13. Categorization of attitude theories by Palmerino et al. ................... 49

14. Study variables (boxes) and research questions (arrows)................ 56

15. Treatment group nomenclature......................................................... 57

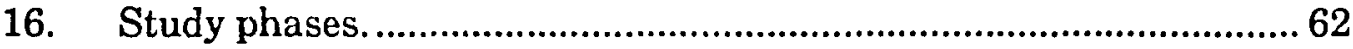

17. The Quality Growth graph — the Beta perspective........................ 75

18. Relationships among variables in quality growth............................ 76

19. Possible to Avoid Bureaucracy, dependent variable D1................... 77

20. Definition of Bureaucracy, dependent variable D2 ......................... 78

21. Opportunities, dependent variable D3 ............................................. 78 
22. Responsibility, dependent variable D4............................................... 79

23. Example Actions, dependent variable D5 .......................................... 79

24. Need for Change, dependent variable D6........................................... 80

25. Partnership, dependent variable D7 .................................................. 81

26. Coaching, dependent variable D8 ....................................................... 81

27. Schedule for the cultural-simulation classes..................................... 83

28. Final storyboard for MGC cultural video............................................8 89

29. Example quality graph at end of Beta game. ................................... 97

30. Simulation class attributes and treatment groups......................... 102

31. Simulation class schedule for each session. ..................................... 107

32. Layout of the simulation classroom................................................. 111

33. Treatment group gender distribution.............................................. 121

34. Subject length of employment and age. ......................................... 124

35. Subject length of employment by treatment group. ....................... 124

36. Mediating variable questions. .......................................................... 131

37. Magnitude of attitude change distributions. ................................... 148

38. Magnitude of attitude change distributions (continued)................ 149

39. Magnitude of attitude change distributions (concluded)................ 150

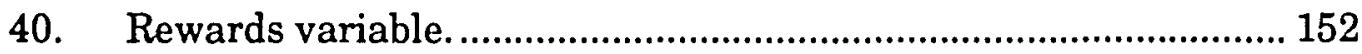

41. Organizational change questions. ................................................. 153

42. Bureaucracy support questions. ..................................................... 154

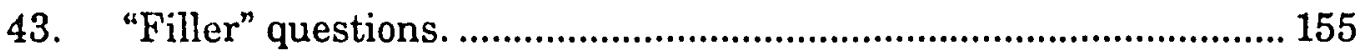

44. Modified causal model...................................................................... 156

45. Summary of Research Question conclusions. ................................ 157 
$\begin{array}{lc} & \text { xiv } \\ \text { FIGURE } & \text { PAGE }\end{array}$

46. Summary of refinements in experimental procedures. ............... 163

47. Range of subject's Perspective Taking scores. ........................... 177

48. Project milestones and activities............................................. 213

49. MGC Acquisition, Employment and Sales History....................... 214

50. Life cycle of the Compute Engine project..................................221

51. Competing values for the second generation products.................225

52. The Hierarchical Model of Knowledge in Ethnoscience............... 227

53. Cultural scenes observed at Mentor Graphics........................... 228

54. Contrasts between the MGC and Tektronix cultures................... 234

55. Attributes of 'bureaucracy'.................................................... 243

56. Attributes of a 'small company atmosphere' (SCA).................... 244

57. Mentor Graphics Statement of Values...................................... 246

58. Frames of Reference defined during the interviews.................... 248

59. The basic model of the WADA simulation...................................2290 
AN ABSTRACT OF THE DISSERTATION OF Terry Robert Schumacher for the Doctor of Philosophy in Systems Science presented June 11, 1992.

Title: Simulation Design, Role Identification and Attitude Change in a High Technology Culture.

APPROVED BY THE MEMBERS OF THE DISSERTATION COMMITTEE:

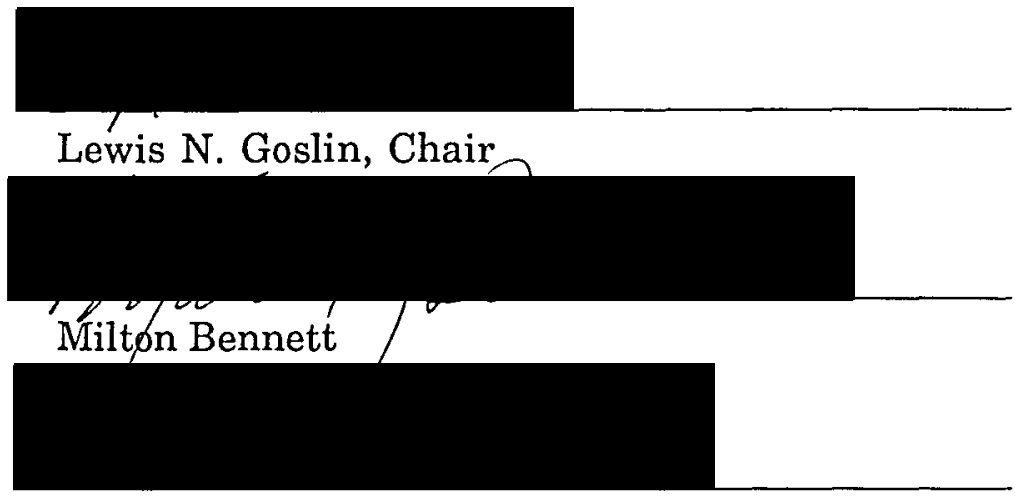

Dean Frost

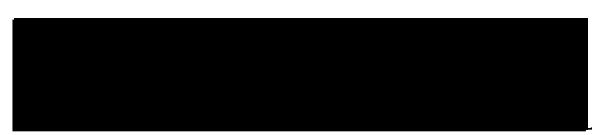

Martin Zwick

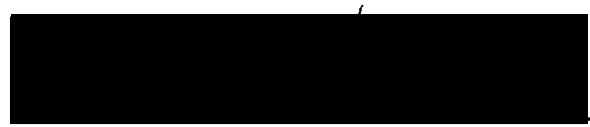

Sheldon Edner

Gaming-simulations (G-S) are those in which participants play roles, make decisions and receive feedback while interacting with a simulation 
model. This study used attitude change to measure of G-S effectiveness in exploring questions relating simulation design and effectiveness.

To define participant attitudes and the content for a simulation, the organizational culture of a software engineering firm was studied using an ethnographic approach. Inconsistencies between the existing culture and expressed ideals were measured using an 40 item attitude questionnaire drawn from statements made during interviews.

Simulation structure and participant cognitive style were factors hypothesized to influence identification with a simulation role. Role identification was hypothesized to influence attitude change. Two versions of the simulation were designed to produce differential role identification. Role identification was measured by having the simulation software ask players questions near the end of the eight hour simulation class. The Davis (1980) Empathy scale was used to measure cognitive style.

The WINNING AT DESIGN AUTOMATION gaming-simulation was created to induce attitude change toward the ideals. The simulation is written in HyperCard. Each participant managed a department, allocating their time to tasks that earned points as they competed in teams.

A control group of 42 employees, and 97 of the 122 who played the simulation, completed pre- and post-simulation questionnaires. There was significant attitude change for all treatment groups. The different versions of the simulation did generate stronger and weaker role identification as predicted. The Empathy scale did predict role identification. The treatment group with highest role identification did not have the greatest attitude change. Other factors influencing the linkage of role identification to attitude change are discussed. 


\title{
CHAPTER I
}

\section{INTRODUCTION}

\section{SIMULATION DESIGN — MOVING FROM ART TOWARD SCIENCE}

\author{
I hear and I forget \\ I see and I remember \\ I do and I understand
}

- Chinese proverb

Simulations offer a number of benefits as techniques for education and training and their use has grown substantially during the past two decades. They can provide participants with experiential learning, suggest alternate cognitive maps to them and expose them to new reward systems in psychologically reassuring environments. Despite the effort that has gone into designing and using simulations, the design of educational simulations remains less a science than an art. The term 'simulation' has different meanings in different contexts. This study addresses a subset of simulations, commonly referred to as 'gaming-simulations', in which participants make decisions and play roles during the simulation. Throughout this discussion the terms 'simulation' and 'gaming-simulation' are used interchangeably. ${ }^{1}$

Greenblat (1988) introduces her book on simulation design by noting how little in the way of published guidelines exist.

1 See Greenblat (1988, p15) or Greenblat and Duke (1981, Chapter 2) for a discussion of the problems of terminology surrounding games, simulations and role playing. See also Cruickshank and Mager (1976). 
The best way to learn about gaming-simulation design would be first to participate in and run a great many gaming-simulations, and second to work directly with an experienced designer on a concrete design problem. Many very capable designers have considerable difficulty giving abstract explanations of how they work; many of them can clearly explain what they are doing and why as they go through the process. (Greenblat 1988:11)

While this "learn by doing" approach is consistent with the beliefs of simulation proponents, certain simulation structures may be more effective than others and testing their effectiveness could result in guidelines for future designs. The question of which structures to test could also be approached in a "learn by doing" manner, or it could be theoretically driven. The approach in the present study is driven by a communication theory as discussed in the literature review.

Part of the difficulty in providing guidelines for simulation design is the multiplicity of effectiveness criteria. Greenblat (1988) lists: increasing motivation, teaching, skill development, attitude change and self-evaluation as objectives that have been considered. Another might be the development of interpersonal relationships among participants. Simulation effectiveness was assessed by measuring attitude change in the present study.

\section{Research Topics Addressed}

Relatively little research has focused on several important topics in simulation design:

1) The question of whether simulation experience leads to attitude change remains clouded, with various studies reporting conflicting findings.

2) Nearly all previous studies were conducted using students as subjects, leaving open to question how far the results could be generalized to non-student populations. 
3) Very few studies have compared different simulation structures for their relative effectiveness.

4) The measurement of subjects' individual differences (e.g., cognitive style, cognitive complexity) to determine whether these have an effect on their assimilation of material presented in a simulation format is an research area that has not been explored.

5) Little research testing theoretical models of attitude change in simulation research has been done. The need for better theory and for linking existing theory to simulation design is acknowledged in the literature.

The present study attempted to increase our understanding of simulation design by addressing each of the preceding topics with the following procedures:

1) A simulation was designed to produce attitude change in a specific target population. The experimental procedures adopted improve upon those of earlier studies.

2) Attitude change data was collected in a high technology corporation and the culture of the corporation was studied to define the social context of the attitudes targeted for change. The question of applicability of simulation research conclusions to nonstudent populations was thereby addressed.

3) This study compares the relative effectiveness of different versions of a simulation in causing attitude change. These versions were constructed so as to minimize differences in content in order to test the relative effectiveness of the different simulation structures.

4) Individual subject's cognitive styles were measured by including subscales of the Davis (1980) empathy instrument in the questionnaire. This allowed tests of hypotheses about the relationship between individual differences in subjects' cognitive style and role identification.

5) A communication theory is linked to the simulation design in the literature review, thus addressing the need to test theoretical models relating simulation design and attitude change. 
How this study addresses each of these research topics can best be understood by considering the evolution of the causal model adopted by simulation researchers. That awareness helps explain the variables measured in this study and expectations about relationships among those variables.

\section{Evolution of the Causal Model}

Publications exploring the relationships between simulations and attitude change reached a zenith in the 1970s and then declined in the 1980s. No clear conclusion emerged about the relationship between simulation and attitude change, and no theory emerged to guide further research toward a clearer understanding of that relationship. Attitude change has previously been attributed to an independent variable 'simulation' as suggested by Figure 1. Typical experimental designs included a treatment group that experienced a simulation and a control group that listened to a lecture on material similar to that in the simulation.

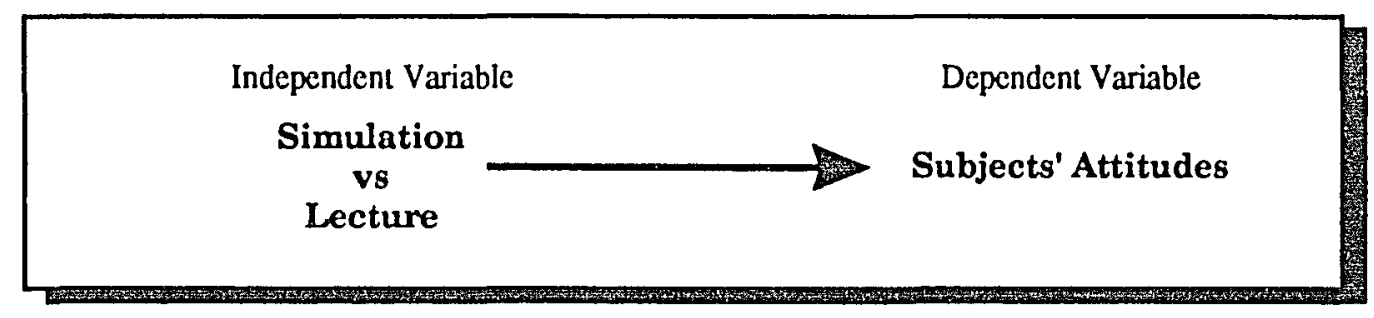

Figure 1. Implicit causal model in previous simulation research.

In the present study, 'simulation' as an independent variable has been separated into the two independent variables and a mediating variable. This conceptualization results in the more complex causal model shown in Figure 2. Each of these variables is described briefly. 


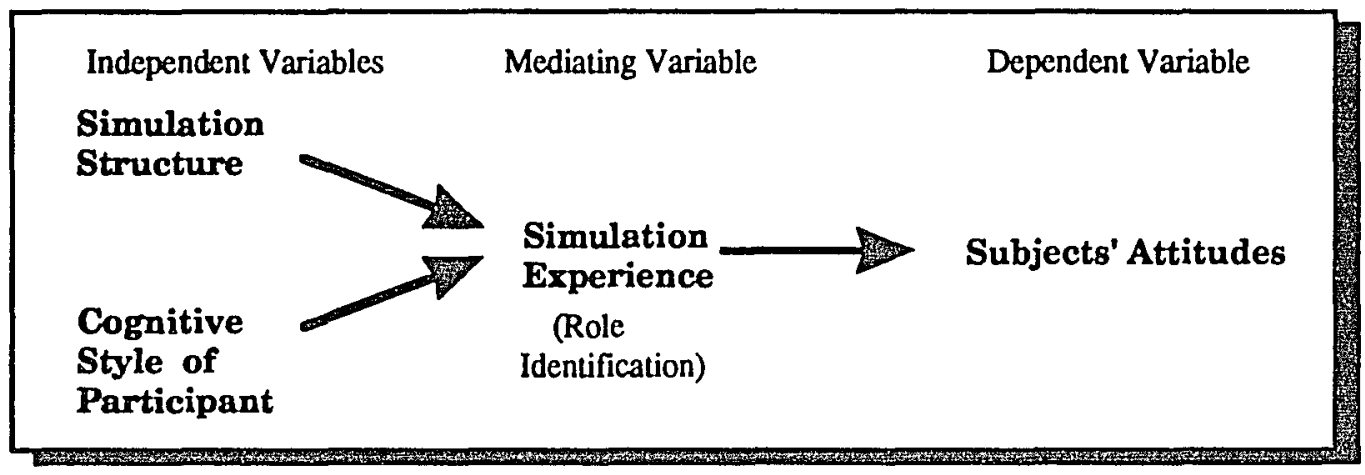

Eigure 2. Hypothesized causal model in the present study.

Simulation Structure. Three treatment groups that experienced different versions of the simulation were the conditions that defined different values for the independent variable 'Simulation Structure'. Certain simulation structures that were present in one version and absent in others were expected to produce more role identification among participants.

Cognitive Style of Participants. A subset of the Davis Empathy Scale (Davis 1983, 1980) was included in the pre-simulation questionnaire to measure the cognitive style of participants. It was expected that people who had higher scores on this cugnitive style measure would be more likely to identify with a role they played in a simulation.

Simulation Experience. That participants in the same simulation have different experiences has been discussed as a possible cause for the variability in results of simulation studies addressing attitude change (Bredemeier and Greenblat 19812). While such differences are obvious to those who have experienced gaming-simulations, previous research designs have not attempted to measure 'Simulation Experience'. Differences in

\footnotetext{
2 Their comment on this issue is quoted in the literature review.
} 
individuals' simulation experience could be measured along many dimensions. The Constructivist communication theory adopted in this study suggests several that could influence attitude change. Role identification is one of these dimensions and this study measured how different subjects identified more or less strongly with a role they played in a simulation.

Previous studies have also suggested that participants' role identification during a simulation is a factor influencing attitude change. Near the end of the simulation, participants answered several questions to measure their identification with the simulation role. Their perception of contrast between that role and the culture of the client organization was a second mediating variable collected to measure 'Simulation Experience'. Participants who had higher levels of role identification during the simulation were predicted to be more likely to demonstrate attitude change. Greater perceived role-culture contrast was also expected to lead to greater attitude change.

\section{REQUIREMENTS AND STRUCTURE OF THE STUDY}

A study testing the effectiveness of simulation structure and the relevance of participants' cognitive style entails the following requirements. Attitude change can be best measured by comparing participant attitudes before and after a simulation. Participants as well as an instrument to measure attitude change are therefore necessary. Also needed is a simulation that incorporates the structural attributes hypothesized to cause attitude change, and content that addresses the attitudes to be measured. The simulation should be designed so that these structural attributes can be 
presented differentially across different versions, allowing different treatment groups to experience different simulation structures.

One obvious approach to meeting these requirements was to find an organization willing to have a simulation developed for use in its internal training programs. While many different topics can be addressed with simulations in organizational development work, it was believed that there was some synergy in selecting the organization's culture as the topic for the simulation. The study design was to embed the simulation research within an assessment of an organization's culture as diagrammed in Figure 3.

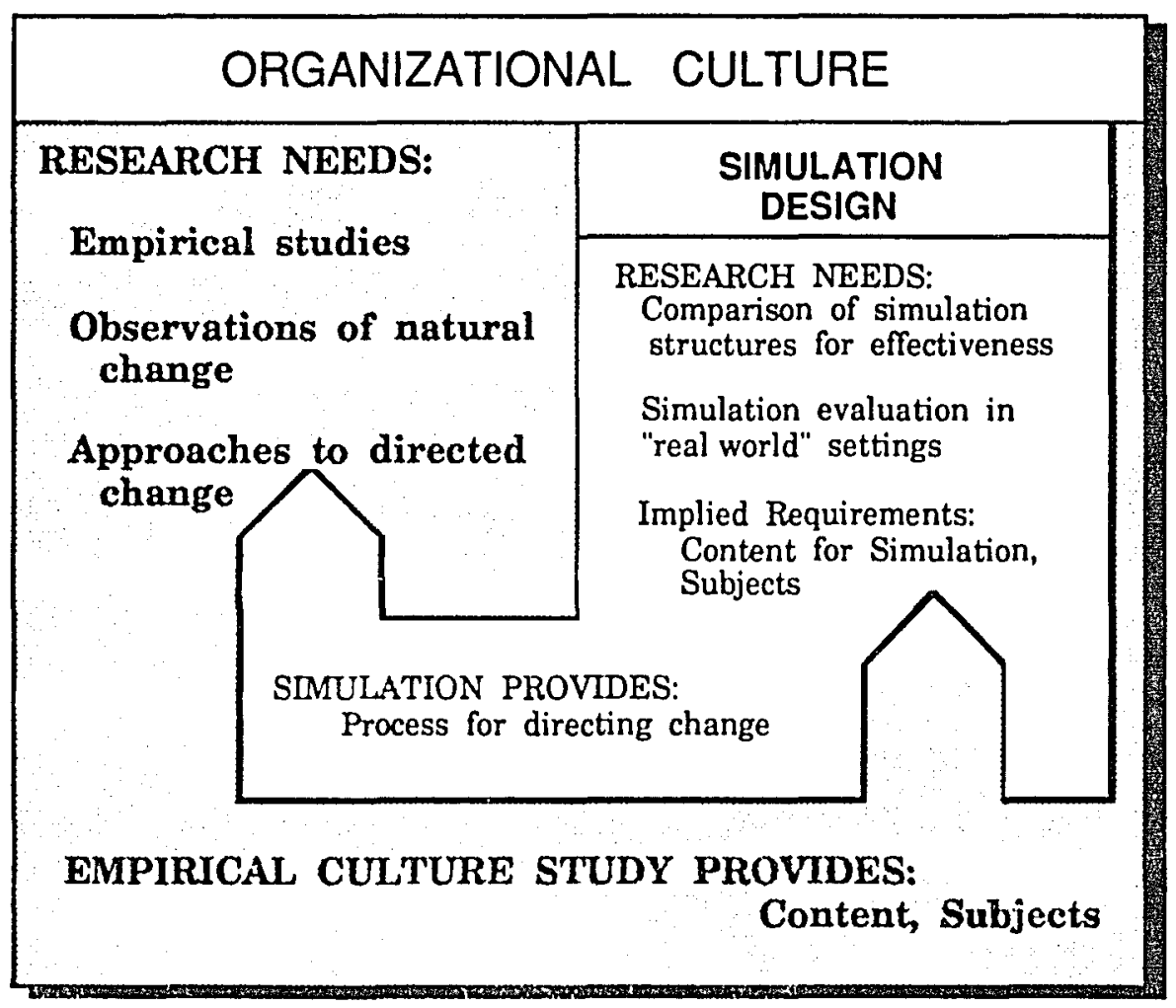

Figure 3. Diagram of research topic integration.

The study of organizational culture saw rapid growth during the 1980s. The requirements for building the simulation included gathering a significant 
amount of data about a topic. If that topic were an organization's culture then the data collected might represent a contribution to this literature.

\section{Project Scope and Activities}

Morey and Luthans (1985:223) recommend that research on organizations should have a two-stage approach:

In the initial stage of research, a cluster of methods that could be broadly termed ideographic/ qualitative/ insider become particularly useful for organizational studies. In the later stage, a seemingly opposite cluster of analytical approaches broadly labeled nomethetic/ quantitative/ outsider become the focus.

The present study adopted an approach consistent with this recommendation. A qualitative study of an organization's culture was made to define a context for a quantitative study of the effectiveness of simulation design. Figure 4 summarizes the project's major activities and outputs. The qualitative description of the culture (stage one in Morey and Luthans' approach), occupied the first phase of the study. Morey and Luthans' 'later stage" is divided into Phases 2, 3 and 4. The four phases in Figure 4 will be referred to throughout this thesis, and are used to structure the Methodology section (chapter IV).

Phase 1 focused on qualitative data collection and was composed of activities to immerse the investigator in the organizational environment. The output of this phase included many statements of attitude and belief made by members of the organization. These formed the basis of the questionnaire developed in Phase 2.

In contrast to Phase 1 , the focus of Phases 2 and 3 was design. Phase 2 structured the data from the interviews into frames of reference - systems of belief that were the conceptual structures that the simulation would convey 
to participants. Also included was the development of a questionnaire for measuring attitude change. Phase 3 translated the frames of reference into the concrete formats of a computer simulation, roles and a video tape.

\begin{tabular}{|c|c|}
\hline Activities & Outputs \\
\hline $\begin{array}{l}\text { Phase 1. Qualitative Data Collection. } \\
\text { Interviewing, Participant Observation, } \\
\text { Reading printedmaterials }\end{array}$ & $\begin{array}{l}\text { Content for simulation, Statements of } \\
\text { attitude \& belief }\end{array}$ \\
\hline \multicolumn{2}{|c|}{ Phase 2. Frame of Reference \& Questionnaire Design. } \\
\hline $\begin{array}{l}\text { Frame of Reference design, } \\
\text { Questionnaire development }\end{array}$ & $\begin{array}{l}\text { Frame of Reference "Tree" charts, } \\
\text { Questionnaire }\end{array}$ \\
\hline \multicolumn{2}{|c|}{$\begin{array}{l}\text { Phase 3. Simulation Class Design \& Production. } \\
\begin{array}{ll}\text { Simulation design, programming \& testing, } & \text { "Winning At Design Automation" } \\
\text { Video tape scripting \& production } & \text { simulation, Video tape }\end{array}\end{array}$} \\
\hline $\begin{array}{l}\text { Phase 4. Quantitative Data Collection. } \\
\text { Questionnaire administration, Treatment group } \\
\text { determination, Simulation classes }\end{array}$ & $\begin{array}{l}\text { Questionnaire data, Role identification } \\
\text { data, Simulation assessment }\end{array}$ \\
\hline
\end{tabular}

Figure 4. Study phase, activities and outputs.

Phase 4 included the use of the simulation and the collection of quantitative pre- and post-simulation data for analysis. In this phase the investigator returned to the organization and conducted the activities in a traditional experimental design to test the intervention's effectiveness.

\section{SUMMARY OF FINDINGS}

The findings of the study were that:

- In general the causal model in Figure 2 was confirmed, although a second mediating variable, 'Perceived Role-Culture Contrast' emerged as a separate measure of 'Simulation experience'. Different simulation versions did create different levels of the mediating variables, (Role identification and 
Role-Culture Contrast); one measure of cognitive style was a significant factor predicting role identification; role-culture contrast was a significant factor influencing attitude change.

- The causal model was not confirmed in that evidence did not support a relationship between Role Identification and attitude change. What effect this dimension of 'Simulation experience' has on attitude change remains unclear.

- The participants enjoyed the WINNING AT DESIGN AUTOMATION (WADA) simulation created in this study and recommended that others in the organization experience it. As one measure of simulation usefulness, the organization has budgeted for 300 employees to experience the simulation in 1992 , approximately $15 \%$ of the work force.

- Significant attitude change was observed for the "Quality Growth" dependent variable in all treatment groups indicating that the primary design goal of the simulation - causing attitude change - was achieved.

- No difference was observed in the amount of attitude change between the Enhanced Simulation and Video Control groups indicating that the video tape did not measurably influence change in the attitudes measured.

- Subject scores on the "Perspective Taking" subscale of the Davis Empathy Scale was a factor separate from simulation structure that predicted their identification with the simulation role.

- Of the demographic variables analyzed, only "length of employment in the client organization" had a significant relationship with attitude change. Employees who had been with the organization longer showed greater attitude change. 
- The Enhanced Simulation (also used by the Video Control group) caused significantly more role identification than the Simplified Simulation, as measured by subjects' self-reports. There was also greater perceived contrast between the simulation role and the culture of the client organization in the Enhanced Simulation group. Thus the secondary design goal of the simulation - creating versions that produced different 'Simulation Experience' for participants- was achieved.

- Contrary to the study hypotheses, the Simplified Simulation had more attitude change than the Enhanced Simulation and Video Control groups even though this group had lower role identification. The 'Role-Culture Contrast' variable is discussed as a possible explanation for this finding.

- Role-Culture contrast had a non-linear relationship with attitude change. Very low and very high perceived Role-Culture Contrast led to little attitude change while mid-range contrast led to significant change.

- There was evidence that some change in subjects' cognition about the topics addressed by the simulation occurred at a system-level, such as that described by the simulation role. The suggestion is that subjects learned the simulation role and incorporated it into their organizational cognition.

\section{CLIENT ORGANIZATION - MENTOR GRAPHICS (MGC)}

In the summer of 1986 the principal investigator approached several organizations in the Portland area to locate a client willing to participate in the study as outlined in Figure 4. Richard Anderson, then vice-president of Human Resources at Mentor Graphics, indicated that he would support such a study. In November a letter of agreement was signed (Appendix A). The 
project milestones and Mentor Graphics milestones during the course of the study are displayed in Appendix C.

Mentor Graphics was founded in April of 1981 and began operations with nine employees in that year. The Company designs, distributes and services engineering software products used for computer aided engineering (CAE) and computer aided design (CAD). By most measures Mentor Graphics was extremely successful from its founding through 1990 . The company emerged as the world leader in market share for electronic design automation (EDA) software in the late 1980s. A business profile of Mentor Graphics is provided in Appendix C.

At the time the interviewing process began in early 1987 the company had 900 employees. In the third quarter of 1990 , when the simulation classes were held, there were about 2700 employees. Cultural issues surrounding this rapid growth became the focus of the simulation. 


\section{CHAPTER II}

\section{LITERATURE REVIEWED}

The first section of this chapter provides an overview of simulation studies addressing attitude change to identify methodological issues in this field. The second section considers simulation structure studies to summarize the suggestions from previous work about effective designs. The third describes Constructivist models of culture and communication to support a 'system-level' model of frame-of-reference change that guided the simulation design. The final section offers a selective review of the attitudechange literature.

\section{OVERVIEW OF SIMULATION AND ATTITUDE CHANGE STUDIES}

Karl Groos is only one of the authors quoted in Bruner (1976:66) who support the idea that play is necessary for development.

Animals cannot be said to play because they are young and frolicsome, but rather they have a period of youth in order to play; for only by so doing can they supplement the insufficient hereditary endowment with individual experience ...The animals do not play because they are young, but they have their youth because they must play.

Gaming-simulations are one opportunity to incorporate play in education. The comments of Gary Shirts (1976:37), provide some historical perspective on simulation research. Shirts, who is known for having created the BAFA BAFA and STARPOWER simulations in the 1970s, indicates the expectations of early researchers as well as their failure: 
...when simulation games were still a promise, it was possible to argue that they would be so widely used that it would be important to find out if they were worth the effort. But simulation games have not ... overwhelmed the education establishment as many hoped and predicted.... One might expect that after 10 years of research into the effects of simulation games, there would be a satisfactory answer to most basic questions about their use ... Are there substantial differences in the effectiveness of different games? Are simulation games more effective with certain types of students?

Fifteen years have passed since these remarks and answers to those same questions have still not emerged. As Whiteley and Faria (1989:44) note, "Despite the proliferation and widespread use of business simulation games, a review of the literature reveals that the pedagogical value of such games still remains unclear."

Several studies demonstrate that simulation experience can cause attitude change. Pierfy (1977) reviewed 22 studies on the effects of simulations published between 1963 and 1975. Eleven studies reported on attitude change as a measure of simulation effectiveness, eight of which found that simulations were significantly more effective than "conventional instruction". Numerous other studies concluded that no attitude change followed simulation exposure (e.g., Cherryholmes 1966, Jackson 1979, Bredemeier et al. 1982) or that less change occurred than in a comparable lecture-only group (Gray and Walcott 1977). No clear understanding of these inconsistent findings has emerged.

\section{Methodological Concerns}

Pierfy concludes his review with a discussion of methodological deficiencies. These are summarized in Figure 5. He concludes that these concerns need to be addressed before additional studies can develop this field. 
The possibility of developer bias. - In eight of the 22 studies the simulation developer was facilitating a simulation in an experiment to evaluate the simulation he or she had developed.

Use of intact classroom groups as subjects. - All of the studies were conducted in classroom settings. Pierfy finds several problems inherent with this situation. In studies that compared simulations with "conventional instruction" different teachers usually conducted the simulation session and the control group lectures. Teacher ability is therefore an uncontrolled variable. Random assignment of subjects to treatments is also precluded.

Compressed Assessment / Treatment cycle. - This is related to the use of classroom groups as subjects. The pre-test, simulation, post-test research cycle in many of these studies was very compressed, often occurring within a 60 to 90 minute period. Pierfy believes this could lead to the pre-test interacting with the independent variable. Practice effect is also a concern.

Poor reporting of methods. - Some studies explicitly mention that a post game debriefing was conducted prior to administration of the instrument while others make no mention of a debriefing. While the simulations are usually well described, the descriptions of the control treatments have been vague.

Poor reporting of findings. - Virtually all of the studies involved investigator developed instruments but "...precious little information is reported concerning the genesis of the tests." Some studies do not report reliability data and for many of those that do, the reported coefficients are low.

Figure 5. Summary of Pierfy's methodological concerns.

The majority of simulation studies have used students as subjects, including those reviewed by Pierfy (half of which used high school students and half younger students). Besides the issues surrounding the classroom setting he mentions, there remains the question of the applicability of such studies' conclusions for other populations. Barling et al. (1991:725) observe that, "attitudes are most amenable to change during adolescence and early adulthood; susceptibility declines rapidly thereafter, remaining low throughout the remainder of the life cycle." The continued use of young students as subjects would result in research questions being answered for only the most susceptible audience. Although simulations are increasingly 
used in corporate training and development (Jacobs and Baum 1987, Faria 1987) few studies have reported their effectiveness with adult populations (Kaplan et al. 1985 is one exception). While concerns about students as experimental subjects will undoubtedy remain a perennial question in the social sciences, as Chaiken and Stangor (1987:616) note, "...more research on social influence in natural settings ... would be desirable complements to our laboratory-based knowledge".

Butler et al. (1988:4) provide a more recent review of the simulation research, and comment:

It is the authors' opinion that most attempts to show that learning is associated with simulations have fallen short of the mark. ... Two factors in particular seem to have impeded development of sound knowledge in this area: (1) inadequate attention to the design of research studies and (2) the lack of a paradigm to guide the investigation of learning outcomes.

In particular they criticize the use of intact classes to define treatment groups, a practice which they consider a "quasi-experimental" design. They offer criteria for a true experimental design ${ }^{3}$. After surveying articles in Simulation and Games and the Association for Business Simulation and Experiential Learning (ABSEL) proceedings over two four-year periods, they found that only $14 \%$ of the journal articles (total $N=170$ ) and less than $3 \%$ of the proceedings articles (total $\mathrm{N}=458$ ) met their definition of "experimental research designs". Incorporation of the methodological suggestions by Pierfy and Butler would appear to be necessary components of future research.

3 Butler reports that only about $40 \%$ of all journal articles and $20 \%$ of proceedings articles included any research methodology in the 1970s. The rest were theoretical, or either advocated or described simulations with no empirical evaluation. The proportion of journal articles that included research methodology declined to less than $25 \%$ in the $1980 \mathrm{~s}$. 


\section{The Need for Improved Theoretical Models}

This section addresses the inadequacy of theoretical models in previous simulation research, an additional concern of reviewers of this literature. Bredemeier and Greenblat (1981:327) fault previous work for not clearly specifying the causal model directing the research. Much like Butler et al.

\section{they want}

...to emphasize the pointlessness of comparative assessments without clearer specification of any rationale for the outcome... ... No methodological or technical refinements of data analysis ... are likely to substitute for the ... careful specification of the theoretical reason for expecting any effects at all.

Most studies addressing simulation and attitude change have not referenced theoretical models of attitude change, despite the extensive literature ${ }^{4}$. The rarity of theoretical models in empirical simulation studies and the absence of any debate about alternate models may indicate that those researching simulation effectiveness share an implicit model.

Many authors have discussed simulation theory (e.g., Stopp 1976, Duke 1974, 1980, 1981; Bowen 1987, Thatcher 1990) but these are not directly linked to specific simulation designs or empirical studies. The call for change made by Buttler et al. and Bredemeier and Greenblat is for the union of theory with design in studies that test simulation effectiveness.

Few discussions in the simulation literature consider whether different simulations can be viewed as a homogeneous treatment. This indicates that

4 The applicability of attitude-change models in social psychology to simulation studies will be considered in the final section of this chapter. If simulation designers do not reference this literature because they believe that other causal models are a better description of the processes encountered in simulation environments, then those alternative mudels become the ones to be discussed and tested. 
the assumed causal model is the "Previous Simulation Research" in Figure 6, where "Simulation" is an undifferentiated independent variable causing change in the dependent variable, "Attitudes". This model suggests the dominant experimental design - one treatment group experiences a simulation while another hears a lecture on the simulation topic. The primary research question in these studies addresses whether simulations are more effective than "conventional instruction".

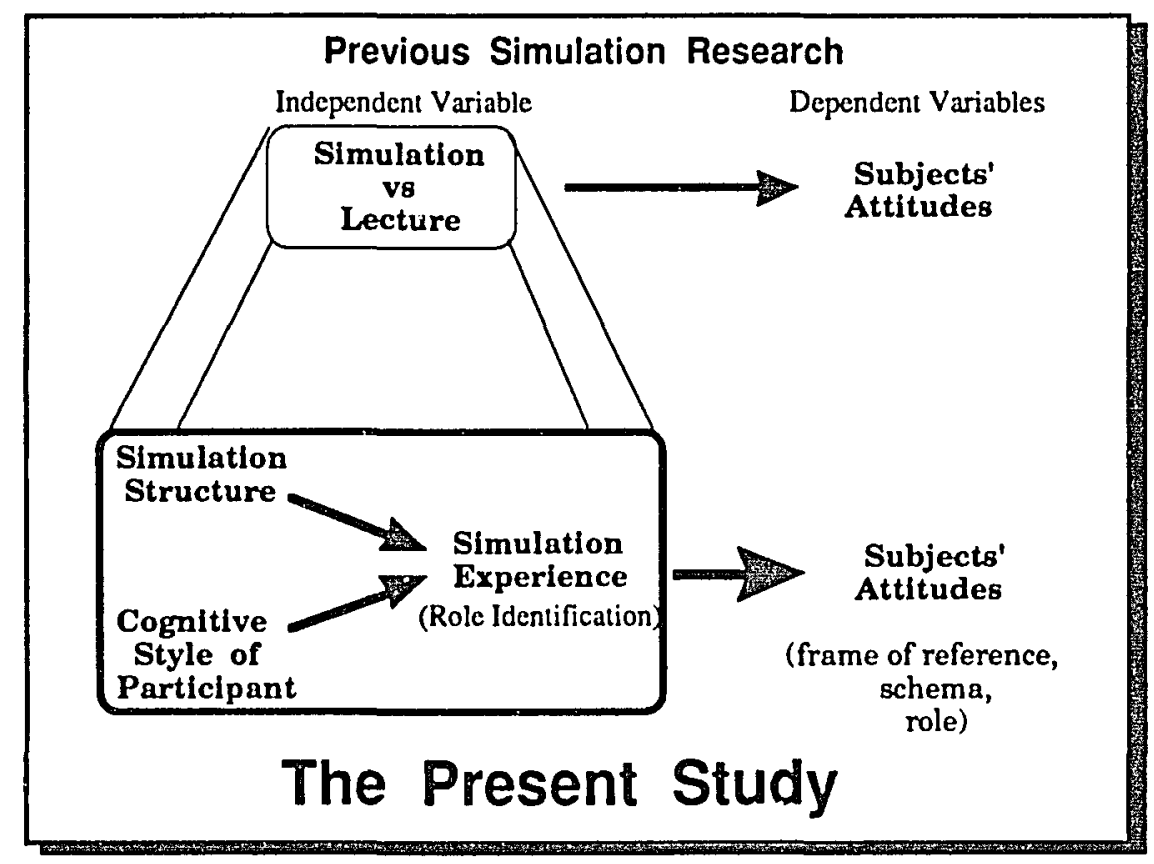

Figure 6. Comparison of causal models.

The need to rethink this causal model was suggested by Bredemeier and Greenblat in their review (1981:307-308).

The most important recent clarification of assessment efforts has been recognition of the many complex ways in which a given game is not the same experience for everyone and may not be the same experience for anyone... The tendency to think and write about the learning from the experience of $a$ game must give way... to recognition that what anyone learns from any experience depends on a host of circumstances... 
Chief among the circumstances Bredemeier and Greenblat list as fertile ground for research are "the nature of the persons", "internal game variables", and "the shape of the experience". They call for future studies to address these in assessing the effectiveness of simulations. The causal model adopted in the present study (Figure 6) has evolved from the previous one, and it hypotheses either an independent variable or a mediating variable for each of Bredemeier and Greenblat's three "circumstances". Under their heading "the nature of the persons" they discuss participant cognitive style. The variable 'simulation structure' is intended to address their "internal game variables", while the mediating variable 'simulation experience' is an attempt to measure what they call "the shape of the experience".

Comparative studies of simulation structures could demonstrate how different structures effect a simulation's effectiveness. As these relationships become better understood, more effective simulations can be built. Then researchers can more clearly assess whether simulations (with effective structures) are better than other teaching methods. The few studies that have reported work on the effectiveness of simulation structures are reviewed in the following section.

\section{SIMULATION STRUCTURE STUDIES}

Simulations discussed in the literature vary dramatically in content and structure. Little work has been done on developing a taxonomy of gamingsimulations. Lester and Stoil (1979) suggest a two-category system, "role specific" and "role generic" simulations. Thornton and Cleveland (1990) present the five-category continuum in Figure 7 though two of these "simulations" are variants of role playing and do not involve any simulation 
model external to the participants. Clemens (1976) lists four types of simulations used in teaching political science. None of these represents an accepted taxonomy of simulations. Bredemeier and Greenblat (1981:311) observe "...that some general taxonomy of games is needed to enable designers to relate game components to specific goals." This void has likely contributed to the lack of research on the relative effectiveness of different simulation structures. The previous work that suggested structural attributes which were included in the design of WINNING AT DESIGN AUTOMATION, the Mentor Graphics Culture simulation, are discussed next.

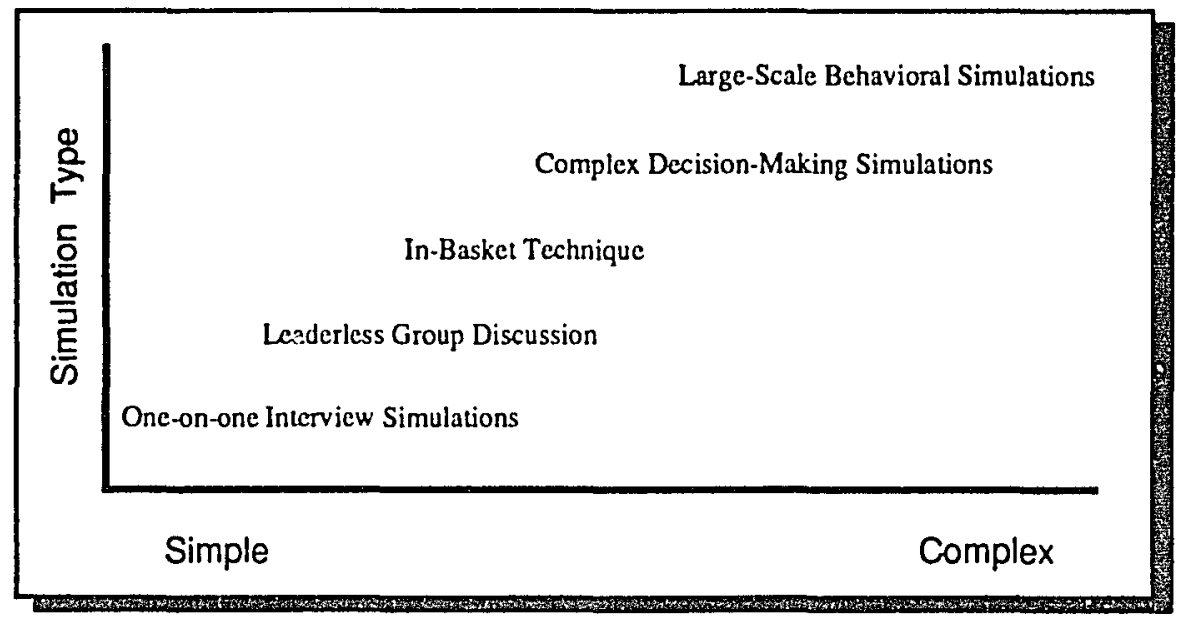

Figure 7 Thornton and Cleveland's simulation continuum.

\section{The Fletcher Study}

Fletcher (1971) conducted a study in which fifth-grade students played the CROSSING PLACE GAME, which teaches how Eskimos hunt caribou. Students played the game six or seven times as they tried to improve their scores. In this game, the participants do not adopt roles and the information 
the simulation is intended to convey is contained in the most successful strategy ${ }^{5}$. Fletcher's view that a simulation's successful strategy is the primary knowledge it will convey may seem an obvious approach to simulation design. However Schild (1968:144) noted that this has not been a widely adopted approach, an observation that remains accurate today.

It is somewhat surprising that studies of learning in games have not paid much particular attention to (strategy). The variables by which "learning" has been measured have typically been unrelated to behaviors needed for winning; rather, it has been expected that the players learn several other contents, even if these are not necessary for successful play.

The Fletcher study included two independent variables which he labeled "game design features". These were the presence (or absence) of record sheets displaying performance in previous games, and the presentation (or not) of the maximum possible score to the students before they played the game. Fletcher analyzed several different types of questions (factual knowledge, awareness of correct strategy, understanding of the structure of the game and attitudes) as well as game performance (points scored) as dependent variables. He concluded that including the record sheets and encouraging students to review their past performances and plan for the next game significantly increased their learning. Fletcher (1971:285) observed that few simulations incorporate this design feature and,

...it would appear that a substantial increase in (simulations) power as teaching devices could be brought about by providing a recording mechanism and providing for multiple plays.

5 Other ways in which simulations could deliver knowledge include embeding it in a role, or in the causal relationships that constitute the simulated environment which participants discover. Another possibility is that the knowledge can be structured so that it emerges during the interaction of roles as described for the BAFA BAFA simulation. 
Gary Shirts' BAFA BAFA

An example of this lack of focus on strategy is the popular BAFA BAFA simulation (1977). Participants are divided into two groups, each of which learns a cultural role (Alpha or Beta) to play as they interact. (The term "cultural role" is used here to indicate that a role has been created for many players to learn and adopt during the simulation. It is contrasted to a "role" which is specific to an individual player.)

Unlike the CROSSING PLACE GAME in Fletcher's study, there is no 'strategy' to play in BAFA BAFA, there is no score, and no winners or losers announced at the end of the simulation ${ }^{6}$. Discovery of the other culture and the experience of culture shock are the intended outcomes ${ }^{7}$. Jackson (1979) and Bredemeier et al. (1982) both failed to demonstrate attitude change using this simulation, though Noesjirwan (1979) was successful. Neither measured whether the participants learned the cultural roles, but instead looked for change in "dogmatism" and "ethnocentrism"; abstract concepts not directly addressed in the simulation. This expectation of change in abstract concepts that are not directly referenced in the simulation is common in simulation research. The lack of significant results in simulation studies may be due to poor specification of the intended results rather than ineffectiveness of the simulation structure.

6 Players in the Beta culture do score points in BAFA BAFA, but this occurs at a micro level and players in the Alpha culture do not score points. The difference between the cultures is how they relate to others. The focus of the simulation is not on teaching a strategy to learn or improve during the simulation. Players do not have a metric for judging their success at the end of this simulation.

7 One observation is that even with well-conducted debriefings, some players remain unaware of the cultural role they did not play in BAFA BAFA. This contributed to the decision to have sequential games, each with a different cultural role, in the WADA simulation. 
Because no external model provides feedback to participants in BAFA BAFA, some researchers would classify it as an elaborate role play and not a simulation-game. In contrast, Thornton and Cleveland (1990) include two types of role play at the low end of their simulation complexity continuum (see Figure 7) and BAFA BAFA is commonly mentioned as a good example of a simulation-game.

Regardless of BAFA BAFA's classification, the presentation of contrasting cultural roles it suggested was a structural attribute suggested by previous simulation research. Fletcher's idea of multiple plays where participants formed and evolved their own strategies required a long playing time. He reports participants' scores still improving after seven games. A modification of this design has the presentation of a non-optimal strategy as a role in an initial game and then the optimal strategy in a second (final) game. This allows the evolution of strategies to be dramatically compressed.

\section{The Livingston and Kidder Study}

Livingston and Kidder (1973) conducted what can be termed an 'ablation study' in which groups of high school students were exposed to complete or partial versions of the DEMOCRACY simulation. The authors' primary purpose was to determine whether "role identification" or "simulation structure" was responsible for the simulation-induced attitude change. To test this question, they removed what they considered the relevant game structure, the scoring system, from the simulation for one treatment group (labeled "simulation structure"). For another treatment group (labeled "role identification") they removed all references to politics and legislation. In addition, one group played the intact simulation and a "control treatment" group played a simulation on an unrelated topic. 
Simulation playing time was closely controlled (about 70 minutes) and no post-simulation debriefing occurred. Attitudes were assessed with twelve questions after the simulation. The mean scores were higher in the completesimulation group than in those in the other treatment groups and these in turn were higher than the scores of the "control treatment" group. The authors conclude that both role identification and simulation structure contribute to the game's effect. Livingston and Kidder offer no logical or theoretical explanation as to why the incomplete simulation versions would be less effective than the complete simulation, or how role identification influences attitude change. In their words:

The reasons for the effectiveness of these features of the game remain a matter for speculation. The effect of role identification may be the result of the player's tendency to empathize with his assigned role... (p. 142)

Controls: Simulation or Information Content. It is significant that, unlike other simulation researchers, Livingston and Kidder focus on the effect of different simulation structures and not on the relative effectiveness of one learning format (simulation) versus another (lecture). In comparing simulation structures, they imply a refinement of the independent variable 'simulation' to 'simulation structure', a step beyond previous research.

Consistent with this change in theoretical perspective they adopted a different methodology regarding control groups. They used a "control treatment" group which played an unrelated simulation. Apparently they believed that an appropriate control in this attitude-change study was the experience of a simulation and not the presentation of information contained in the treatment simulation in a non-simulation format. A few other studies 
have adopted this control structure ${ }^{8}$. The use of a control with simulation experience - albeit containing different information - could result from the researchers' concerns that something beyond the information in a simulation setting (e.g., group processes, active mental exploration, a change in daily classroom routine) could lead to attitude change. Such a control would then be needed. Unfortunately, these researchers did not elaborate on their reasons for choosing this design. There is always likely to be some blurring of content and structure of a simulation when researchers attempt to remove a structural element to test its effectiveness.

Shortcomings. Livingston and Kidder used students in classroom settings as subjects. A different teacher led each class as a treatment group and thus teacher ability is an uncontrolled variable. Also, they measured attitude change by comparing mean scores on a single post-simulation questionnaire, rather than a comparison of pre-simulation and postsimulation assessments. This fails to meet the criteria suggested by Butler et al. (1988) for a true experimental design.

Finally, the "role identification" variable was determined solely by the treatment condition (simulation version). No measurement of subjects' identification was made. This ignores any possible differences between subjects or whether "role identification" ever occurred. It also blurs the definition of the other independent variable "simulation structure". Their study design suggests that the reference to real-world roles hypothesized to

8 Jackson (1979) uses a similar "control simulation" group in her design, and refers to the group playing a simulation with unrelated content as a "Hawthorne Group". Lee and O'Leary (1971) also had a control group play another simulation. These studies represent an interesting departure from the more common use of a lecture on similar material as a control. 
produce "role identification" is only another attribute of simulation structure. If experiencing a modified simulation is the sole determinant of "role identification", perhaps a better label for this group would be "role exclusion". This would convey that the simulation structure was modified to eliminate the specific roles present in the complete DEMOCRACY simulation. The "simulation structure" variable could then be re-labeled "scoring system exclusion". From this perspective both of Livingston and Kidder's two independent variables fit within the category "simulation structure" and attributing an effect of the simulation to "role identification" seems unwarranted.

Both the Fletcher and Livingston and Kidder studies pioneered research into simulation effectiveness by comparing different simulation structures. Why so few others have continued this promising direction is unknown. Perhaps in the waning of studies evaluating simulations (footnote 4) it has merely been overlooked.

\section{The Williams Studies}

During the 1980s, Williams was the most active researcher examining the effect of simulations on attitude change (Williams 1980, Williams et al. 1986, Williams and Williams 1987). These studies are notable for testing explicit theoretical models of attitude change, some of which are borrowed from the social psychology literature.

In these studies, Williams worked with the NOBLES simulation that he developed. The subject matter concerns imaginary lords and knights negotiating over water rights, gold, land, wheat and other economic possessions. Williams measures the attitudes of subjects about the lords, 
before and after they play the role of one of the lords. Subjects in all three studies were college students enrolled in introductory psychology courses.

In the first study, Williams (1980) attempted to test whether Incentive or Cognitive Dissonance theory was the best model for simulation-induced attitude change with a two-treatment group design. Results were inconclusive. In post-simulation interviews Williams observed frequent reports of identification with the nobles and concluded that future research should explore the relationship of role identification to attitude change.

In the 1986 study, Williams et al. again use the NOBLES simulation. As they state,

The term "identification theory" has been coined to represent the belief that a subject's identification with a character is the most important requirement for changing attitudes in a simulation. Specifically, it is suggested that when players develop a powerful identification with a character, the player's self-image becomes intertwined with that of his or her role to the extent that he or she experiences a personal empathy with the character. (page 31)

The study design used four treatment groups and a control group. Two incentive and two dissonance treatment groups received either a "low directiveness" or "high directiveness" condition introduced by instructions from the instructor. For the high-directiveness groups, these instructions suggested that: 1) subjects should not simply play the game but should "see yourself as actually becoming the noble you represent", 2) they should call themselves by the name of their character during the game, and 3) "reminding players as they completed the post-game questionnaire that they had been the noble they were now rating." These instructions were omitted for the "low directiveness" groups. The percentage of subjects in each group reporting identification in post-simulation interviews was recorded. 
Each of the four treatment groups showed significant attitude change though no significant difference in attitude change for incentive vs dissonance groups or for high vs. low directiveness groups was recorded.

More recently, Williams and Williams (1987) had students play several different versions of the NOBLES simulation, which they hypothesized would differentially support role identification. The study design had three treatment groups and no control group; participants' role identification during the simulation was assessed in the next class meeting. Attitude change occurred in the group expected to have the greatest role identification, but not in the other two groups. In their conclusions the researchers raise the question of whether some threshold of identification exists beyond which substantial attitude change occurs and below which there is none.

Several methodological questions can be raised in reviewing the Williams' studies. The issues raised above concerning the use of students and classes as treatment groups apply. In addition, the assessment of role identification in interviews several days after the simulation leaves open questions about students' memory, the possibility of interviewers' cueing subjects about what is expected, inter-interviewer reliability, etc. His analysis of change scores and of the number of subjects reporting role identification at the group level fails to consider individual differences in identification that may exist. The possibility that some factor other that simulation structure could influence role identification has not been considered. A final concern is with the content of the attitudes (dependent variables). That concern will be addressed in the discussion of research on attitude change. 


\section{Review of Simulation Structure Studies}

Tremendous diversity exists in the structure of simulations developed during the past three decades. No accepted taxonomy of simulations has emerged, nor is there agreement on the criterion for effectiveness. Perhaps because of this, little work has sought to clarify the relative effectiveness of simulation structures. The studies reviewed in this section compared structural attributes of simulations for effectiveness in changing attitudes. They offer evidence that including the attributes in Figure 8 in a simulation make it more effective in producing attitude change.

\begin{tabular}{|ll|}
\hline Simulation Structural Attributes & Researcher \\
\hline Multiple plays, inclusion of recording mechanism & Fletcher (1971) \\
Encourage players to explore different strategies & Fletcher (1971) \\
Inclusion of specific roles & Livingston \& Kidder (1973) \\
Inclusion of a scoring system & Livingston \& Kidder (1973) \\
Experience contrasting rolcs & Shirts (1977) \\
Instructions to identify with role & Williams (1986) \\
Subject's use of the character's name & Williams (1986) \\
Freedom for subjects to act differently from the character & Williams (1987)
\end{tabular}

Figure 8. Summary of suggested simulation attributes.

The work summarized in Figure 8 suggests that identification with a role may be a factor leading to attitude change. Research on this model is in its infancy. To date there has been no attempt to measure individual participants' role identification or a demonstration that differential role 
identification between treatment groups has been produced, though two researchers have assumed the latter.

\section{The Interpersonal Reactivity Index - Empathy}

In previous work on simulations and attitude change, there has seldom been control for differences between individual subjects. Bredemeier and Greenblat (1981) suggest that one topic needing research is how subject's cognitive style, one element they discuss under the heading "the nature of persons" ${ }^{n}$, influences reactions to simulations. A logical next step in unfolding the question of the effect of simulation on attitude change is to include some measurement of participant cognitive style that relates to "role identification ability" or empathy or "perspective taking".

Davis $(1983,1980)$ used factor analysis to develop the Interpersonal Reactivity Index (IRI) from questionnaire items drawn from a review of previously published empathy instruments. Davis (1980) also refers to the IRI as the "Multi-Dimensional Empathy Scale". The IRI contains four separate subscales; "Perspective Taking" and "Fantasy" subscales considered primarily cognitive and the "Empathic Concern" and "Personal Distress" subscales affective in nature. Each subscale contains seven questionnaire items. Davis reports acceptable internal reliability measures, for "Perspective Taking" a standardized alpha of .71 and for "Fantasy" alpha of $.78(\mathrm{~N}=447)$. Test-retest correlations ranged from .61 (PT) to $.81(\mathrm{~F})$ with 60 to 75 days between tests $(\mathrm{N}=109)$.

While the IRI has not been previously used in simulation stucies it has been adopted in psychological research (e.g., Long and Andrews 1990). It appears to be a reasonable starting point for assessing individual differences in research on simulation-induced attitude change. The "Perspective Taking" 
subscale appears a priori to have potential use in exploring role identification.

William's "Identification Theory" is the only explicit causal model in the simulation literature relating simulation to attitude change. It is consistent with the evidence from the few other studies reporting on the effectiveness of structural attributes and with some reports on participant cognitive style. While it is an obvious point of departure for future research on simulation effectiveness, the model Williams suggests is not elaborate.

William's model has similarities to a model of communication discussed by Bennett (1979). The Bennett model views the basic process of communication as the "imaginative participation in a different world view". Bennett calls this "empathy". This corresponds to William's use of role identification in a simulation context. Linking Bennett's model to simulation design choices would provide a richer theoretical base to guide simulation research.

Bennett's work falls within the Constructivist philosophy of communication, as does the Ethnoscience approach that was used in studying the culture of Mentor Graphics. The following section provides an overview of Constructivism.

\section{CONSTRUCTIVIST MODELS OF CULTURE AND COMMUNICATION}

Man by his nature is metaphysical and proud. He has gone so far as to think that the idealistic creations of his mind, which correspond to his feelings, also represent reality. 


\section{Qverview of Constructivism}

Constructivism is the theoretical perspective adopted in Phases 1 and 3 of this study (see Figure 4, page 9). Its assumptions contrast with those adopted in Phase 4 where the Logical-positivist approach framed the quantitative data collection and hypothesis testing. The following discussion of Constructivism is included in order that the reader can better understand Ethnoscience, a school within Constructivism which served as a guide for collecting the qualitative data about Mentor Graphic's culture (Phase 1) and Bennett's model of communication which provided support for the "systemlevel" model of change used to guide simulation structure design choices (Phase 3).

Constructivism views reality as a social construction, and is one of several approaches used in social science research (see Figure 9 which is modified after Morgan and Smircich 1980). Morey and Luthans $(1984,1985)$ suggest using an initial quantitative analysis to bound quantitative studies in organizational settings and the Ethnoscience methods are consistent with their suggested initial analysis. It is also consistent with the call of Morgan and Smircich (1980), among others, for more qualitative research in the study of organizations.

The core assumption of a Constructivist approach is that the most relevant 'reality' in the study of social systems is the subjective one created by persons in those systems. A range of opinions exists within the Constructivist literature ${ }^{9}$ on the epistemology of the 'objective reality' which

9 Many writers have adopted the philosophical position of Constructivism, though they do not all refer to themselves as "Constructivists". Authors who have contributed to this - continued on next page - 
is the focus of the natural sciences (e.g., Watzlawick 1984). All

Constructivists agree on the primacy of the 'emic', or insiders' perspective over 'objective reality'. Reality as differently defined by individuals. People respond to their perceptions, interpretations and mental models of the world around them to such an extent that these perceptions actually become the 'reality' that is the basis for their behavior.

\begin{tabular}{|c|c|c|c|c|c|c|}
\hline \multirow[b]{3}{*}{$\begin{array}{l}\text { Core } \\
\text { Ontodogical } \\
\text { Assumptions }\end{array}$} & \multicolumn{2}{|c|}{$\begin{array}{l}\text { Subjectivist } \\
\text { Approaches to } \\
\text { Social Science }\end{array}$} & & & \multicolumn{2}{|c|}{$\begin{array}{l}\text { Objectivist } \\
\text { Approaches to } \\
\text { Social Science }\end{array}$} \\
\hline & & $\begin{array}{c}\text { Constructivism } \\
\text { Phase } 13\end{array}$ & & & & $\begin{array}{c}\text { Logical } \\
\text { Positivism } \\
\text { Phase } 4 \\
\end{array}$ \\
\hline & $\begin{array}{l}\text { reality as a } \\
\text { projection of } \\
\text { human } \\
\text { imagination }\end{array}$ & $\begin{array}{l}\text { reality as a } \\
\text { socia! } \\
\text { construction }\end{array}$ & $\begin{array}{l}\text { reality as a } \\
\text { realm of } \\
\text { symbolic } \\
\text { discourse }\end{array}$ & $\begin{array}{l}\text { reality as a } \\
\text { contexual field } \\
\text { of information }\end{array}$ & $\begin{array}{l}\text { reality as a } \\
\text { concrete } \\
\text { process }\end{array}$ & $\begin{array}{l}\text { reality as a } \\
\text { concrete } \\
\text { structure }\end{array}$ \\
\hline $\begin{array}{l}\text { Arsumpllons } \\
\text { About } \\
\text { Hurnan } \\
\text { Nature }\end{array}$ & $\begin{array}{l}\text { man as pure } \\
\text { spirit, being, } \\
\text { consciousness }\end{array}$ & $\begin{array}{l}\text { man as social } \\
\text { constructor. } \\
\text { the symbol } \\
\text { creator }\end{array}$ & $\begin{array}{l}\text { man as an } \\
\text { ector, the } \\
\text { symbol user }\end{array}$ & $\begin{array}{l}\operatorname{man} \text { as an } \\
\text { information } \\
\text { processor }\end{array}$ & $\begin{array}{l}\text { man as an } \\
\text { adaptor }\end{array}$ & $\begin{array}{l}\text { man as a } \\
\text { responder }\end{array}$ \\
\hline $\begin{array}{l}\text { Some } \\
\text { Favored } \\
\text { Metaphors }\end{array}$ & transcendental & $\begin{array}{l}\text { language } \\
\text { game, text, } \\
\text { accomplish- } \\
\text { ment } \\
\end{array}$ & $\begin{array}{l}\text { theater, } \\
\text { culwre }\end{array}$ & cybernetic & organism & machine \\
\hline $\begin{array}{l}\text { Basle Epls- } \\
\text { temological } \\
\text { Stence }\end{array}$ & $\begin{array}{l}\text { to oblain } \\
\text { phenomenolog } \\
\text {-ical insight. } \\
\text { revelation }\end{array}$ & $\begin{array}{l}\text { to understand } \\
\text { how social } \\
\text { reality is } \\
\text { created }\end{array}$ & $\begin{array}{l}\text { to understand } \\
\text { pattems of } \\
\text { symbolic } \\
\text { discourse }\end{array}$ & $\begin{array}{l}\text { to map } \\
\text { contexis }\end{array}$ & $\begin{array}{l}\text { to study } \\
\text { systems, } \\
\text { process, } \\
\text { change }\end{array}$ & $\begin{array}{l}\text { to construct } \\
\text { a positivist } \\
\text { science }\end{array}$ \\
\hline
\end{tabular}

Figure 9. Perspectives in social science, from Morgan and Smircich.

Another issue on which Constructivists differ is their assumption of the time stability of these created realities. Authors who describe cultures or social institutions (e.g., Spradley 1979, Berger and Luckman 1966) tend to

literature include Kelly (1955), Goffman (1959), Berger and Luckman (1966), Blumer (1969), Watzlawick (1984) and Maturana (1987). 
attribute extended stability to these realities, while those who focus on the individual level of analysis (Kelly 1955, Bennett 1977) stress their continual re-creation. The latter perspective obviously offers more optimism for the therapist or simulation designer. The work of two Constructivist authors as it contributed to this study follows.

\section{Ethnoscience}

In Anthropology the Ethnoscience school (Spradley 1979, 1980) is clearly within the Constructivist perspective. This school has its roots in the work of Benjamin Lee Whorf. The Whorf-Sapir Hypothesis, as discussed by Mandelbaum (1949:162) links the use of language to reality construction.

Language is not merely a more or less systematic inventory of the various items of experience which seem relevant to the individual, as is so often naively assumed, but is also a selfcontained, creative, symbolic organization, which not only refers to experience largely acquired without its help but actually defines experience for us by reason of its formal completeness and because of our unconscious projection of its implicit expectations into the field of experience.

Note the statement that language "actually defines experience for us". This is the core of the Constructivist position ${ }^{10}$. A similar statement is George Kelly's (1955) view of humans as constructing mental models that then structure how they perceive the world. For Kelly the basic unit of knowledge is the construct and he emphasizes the active role of the individual in this process.

10 This is in contrast, for example, to the "man the symbol user" or "man as an information processor" perspectives in Figure 9, where the assumption is that man uses symbols (including language) to represent an external reality. 
Man looks at his world through transparent patterns or templets which he creates and then attempts to fit over the realities of which the world is composed. (p. 9).

By construing we mean 'placing an interpretation' ... He erects a structure, within the framework of which the substance takes shape or assumes meaning. The substance which he construes does not produce the structure, the person does. (p. 50)

This fitting of language categories "over the realities" of experience is more active than the model of people 'selecting and filtering experience' often assumed in the information processing perspectives in the center of Figure 9. Constructivists see in the active creation of categories for experience, and the projection of existing categories onto experience, the creation of the individual's reality. Those realities are defined so that some relationships are not seen, and inconsistencies among others remain unexplored. Such creation may occur from habit, or may be outside the awareness of the individual - points that Constructivists acknowledge - but this does not detract from the implications of the core assumption. ${ }^{1 i}$

Ethnoscience places a heavy emphasis on language as the focus of created realities. Spradley and McCurdy (1972:60-61) frequently note that language reflects the constructed nature of social realities.

All knowledge depends on categorization; ... In contrast to popular opinion, categorization is not a discovery of the natural groupings of objects in the environment. It is, rather, an invention of ways to classify and organize experience.

To capture cultural knowledge with as little distortion as possible, Ethnoscience emphasizes recording statements verbatim to discover the informants' category system, their shared reality. Different groups using

11 The discussion of the Mindlessness-Mindfulness perspective below (Palmerino et. al., 1984) directly addresses the issue of an individual's awareness. 
different language are considered different cultures. Spradley's $(1979,1980)$ discussion of English speakers whose use of language is so specialized as to constitute different cultures includes: officers at a police station, cocktail waitresses in a college bar, tramps in the Seattle jail, and a crew on a tuna boat. For Spradley, as for other Constructivists, daily interactions therefore frequently involve encounters with other cultures, other definitions of reality. The goal in the cultural analysis of Mentor Graphics, the was to describe the social reality shared by the members of that organization. That is, to define the insiders' 'emic' perspective which is the Mentor Graphics culture.

The Ethnoscience emphasis on language suggests that in designing any organizational intervention, attention should be paid to the categories of experience that are shared by members of the culture. This implies that to have maximum impact, a simulation should be culturally unique.

Ethnoscience does not suggest a model of change, however. To define a model of change in socially constructed realities the work of Bennett is reviewed.

\section{Bennett's Empathy Model of Communication}

It is generally accepted that simulation experience is intended to convey alternate realities to participants (Greenblat and Duke 1981, Chapters 9 and 10). Previously there has not been a direct linkage of this idea to a theoretical model of: 1) how simulations cause change or 2) specific elements of simulation designs. This study built upon Bennett's model of empathy to define a "system-level" model of change which guided the design of the simulation. That model links 1) the conveyance of alternate realities to participants, 2) a theoretical model of how simulations cause change, and 3) specific elements of simulation design. 
Bennett (1986, 1979, 1977) defines empathy as "the imaginative intellectual and emotional participation" in an alternate reality. He describes it as the most appropriate communication strategy once the Constructivist assumption of multiple realities has been adopted. His six-step model of empathy is presented in Figure 10. Bennett describes this model as "switching frames of reference", a phrase that accentuates the active setting aside of one system of constructs and the adoption of a new one. He suggests that the model can help one person understand how another defines the 'same' situation differently.

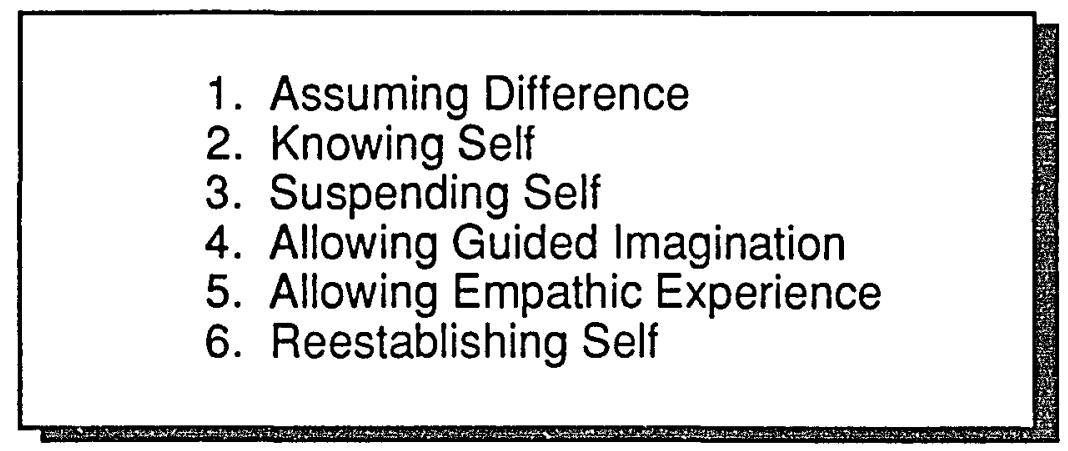

Figure 10. Bennett's six-step model of empathy.

In this model, the suspension of one's normal view of reality is followed by an attempt to experience an alternate reality, after which the initial reality is reestablished. Bennett compares the process to watching a movie in which the viewer imaginatively experiences the alternate reality that the movie conveys. Afterward, the events of daily life reassert themselves and the alternate reality experienced in the movie becomes less salient.

Bennett's model is consistent with the concept of role identification as used in the simulation studies mentioned above. The more detailed description of psychological processes presented by the model offers 
guidelines to simulation design that go beyond simply 'supporting role identification'. It also goes beyond the simple presentation of two contrasting realities that was central to the design of the BAFA BAFA simulation, discussed earlier (page 22).

Each of Bennett's six steps suggests elements for simulation design. The introduction to the simulation could prepare participants to discover differences between alternate realities (step 1 ). The simulation could contain contrasting roles with participants first experiencing a role similar to their normal frame of reference (step 2), being directed to put aside their normal reality (step 3), and then adopting a contrasting role (step 4). An obvious element in simulation is the experience of simulated reality. Bennett's model suggests designing a simulation that encourages participants to create new categories for the experience of the simulated reality (step 5). The simulation debriefing could address how the roles both resembled and differed from the participants' normal frame of reference (step 6). These design structures were incorporated into the simulation created in this study.

Empathy has been considered a mediating variable in attitude change studies. Shelton and Rogers (1981: 366-375) claimed “...there has been no published research on the effects of empathy appeals on attitude change" and used path analysis to confirm "...that empathy-arousing persuasive communications facilitate attitude change." High or low empathy treatments were obtained by reading, or omitting, instructions to "imagine... picture yourself... sympathize... think about ... trade places with" whales displayed in a short film. They concluded that, "...role-taking instructions that arouse empathy can elicit help for an endangered infrahuman species ...". These results offer some support for the causal model adopted in this study. 


\section{Erame-of-Reference Switching, a "System-level" Model}

Bennett and other authors use the term 'frame of reference' in explaining communication processes. It is similar to the concept of 'cultural scene' from Ethnoscience (discussed in the description of Mentor Graphics' culture in Appendix C). Walsh and Charalambides (1990:517) note that many labels are used to refer to such concepts.

The simplified mental representations employed by individuals to give information form and meaning have been variously called belief structures, schemata, implicit theories, cognitive maps, theories of action, world views and assumptions.

Walsh (1990) lists more than 60 authors who together used more than 50 terms to refer to these "simplified mental representations". In this discussion, 'frame of reference' is used to refer to these mental models. Authors clearly consider these mental models to be systems, attributing to them function, structure and resiliency. They are also systems in the sense that they contain identifiable elements and relationships among those elements. The elements include concepts at various levels of abstraction and the relationships between them may be beliefs, attitudes, or values. The literature seldom addresses the question of quantitatively measuring a frame of reference and the present study draws upon the assessment methods from social psychology to measure attitude change as dependent variables, an approach consistent with previous simulation studies. The concept of frame of reference - a system of cognitive elements - was used to guide simulation design.

Although researchers have hypothesized that role identification in simulations is linked to attitude change, no model has been suggested that explains how that identification leads to enduring change. Bennett's 
empathy model offers a detailed description of temporary changing frames of reference - i.e., identification with a role - but he does not discuss how empathy may lead to enduring participant change beyond saying that their increased understanding of the alternate reality can improve communication. He does comment that Step 2, Knowing Self, “...does not eliminate the possibility of change in ourselves as a result of empathizing. It merely makes such change a chosen option rather than an uncontrolable loss." (1979:30).

One possible model for lasting change is that once an alternate frame of reference has been experienced, it can remain latent in the participant's memory. Obvious examples are actors learning scripts or simulation participants playing roles which they can later recall. If the participant later employs a latent frame of reference learned in a simulation setting in other settings, lasting change has been demonstrated. It is suggested that gamingsimulations can be particularly effective in introducing lasting attitude change because of their ability to convey alternate frames of reference that support changed attitudes in that new cognitive system.

Figure 11 illustrates how temporarily experiencing an alternate frame of reference may lead to lasting change using a loose analogy to a thermodynamic model. In this example, two frames of reference organizing similar content are seen as stable, for instance, liberal and conservative political ideologies (dark circles $A$ and $B$ in Figure 11). The forces defining stability of the frames of reference include internal logical consistency, basic values shared by the society, etc., and are measured on the $\mathrm{Y}$ axis (representing free energy in the thermodynamic model).

Subjects are inhibited from moving between frames of reference (adopting perspective $A$ then abandoning it to adopt perspective $B$ ) because 
small changes, such as the piecemeal presentation of bits of information in daily life, do not move an individual over the threshold to a different stable state. (Conversions are rare events.) Change that might be expected from information that challenges a single attitude in a frame of reference is resisted by the related cognitive elements. A small force that moves an individual from $\mathrm{B}$ to $\mathrm{C}$ results in a quick reversal of any effect. Thus frame of reference switching is inhibited in most circumstances. The systemic nature of frames of reference is a force acting to increase the stability of individual cognition.

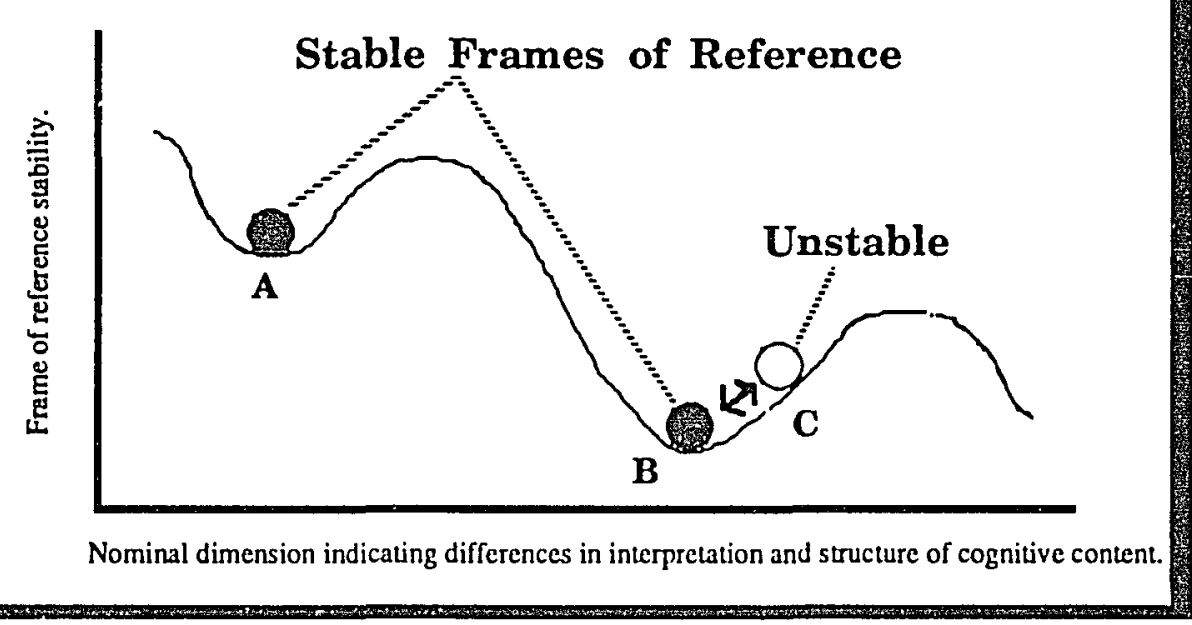

Figure 11. The concept of multiple stable frames of reference.

In this thermodynamic analogy, simulation acts as a catalyst that makes it easier to shift to an alternate frame of reference. An atmosphere of simulation or play suggests less evaluation than normal. Participants more readily adopt what is for them a new frame of reference and are more willing to make the necessary effort to build the connections that define their understanding of the new frame of reference. That is, the simulation 
encourages them to create an alternate reality that is based on the cues presented by the simulation. The simulation also temporarily holds the participant in the new frame of reference while supporting the creation of linkages from it to existing elements in the participant's larger cognitive map. Enduring change can be produced because the newly suggested frame of reference is learned (rather than immediately rejected) and then remains latent in memory, available to the participant as an option to adopt in the future. This explanation describes how the temporary experience of an alternate frame of reference in a simulation can lead to lasting change when the incremental presentation of the same information (elements of a frame of reference) in a non-simulation setting does not produce change.

This model is labeled a "system-level" model of change because it focuses on changing a system of cognitive elements in presenting a new frame of reference (i.e., simulation role). This is in contrast to much attitude change work that explores how exposure to information that varies in a single dimension - e.g., credibility of source, number of arguments, distractions in the environment - relates to change in a single attitude.

The simulation designer may create frames of reference that suggest entirely new realities to simulation participants. This method of inducing lasting change in participants' mental models does not rule out other possible change mechanisms, such as those described in attitude change research.

\section{Simmary of the Contribution of Constructivism}

The "system-level" model of change, which has its roots in Bennett's empathy model, is consistent with, but more elaborate than Williams' "Identification Theory". Each step in Bennett's model suggests structures for simulation design. At the macro level, it suggests the sequential experience 
of two contrasting realities, the first similar to 'self - the normal reality and the second portraying a contrasting 'other' reality.

The Constructivist theory, that individuals create their own realities, and that those realities are structured by language, also suggests that simulations could be more effective by incorporating the language of the participants. Through such incorporation of language, the simulation reality can be more easily integrated into the participants' existing mental models. Ethnoscience, which adopts the Constructivist philosophy, has evolved methods for describing cultures and defining a group's 'normal' reality. Ethnoscience methods were used in the present study to gather data about the client organization's culture for the simulation.

Figure 12 diagrams how adopting a Constructivist perspective embellishes the causal model suggested by Williams' "Identification Theory". In Williams' theory, simulation structure is thought to influence role identification, in turn causing attitude change. This causal model is represented by the three rectangles.

In the present study, role identification is considered to be one dimension of Bredemeier and Greenblat's (1981) more broadly defined "Simulation Experience". The Constructivist assumption of unique individual realities is consistent with their observation that participants vary in what they experience in a particular simulation. This suggests measuring individual's on this mediating variable, a methodological refinement beyond that used by Williams (1987) or Livingston and Kidder (1973). Those researchers implicitly assumed that all subjects in a treatment condition had a similar level of role identification. 


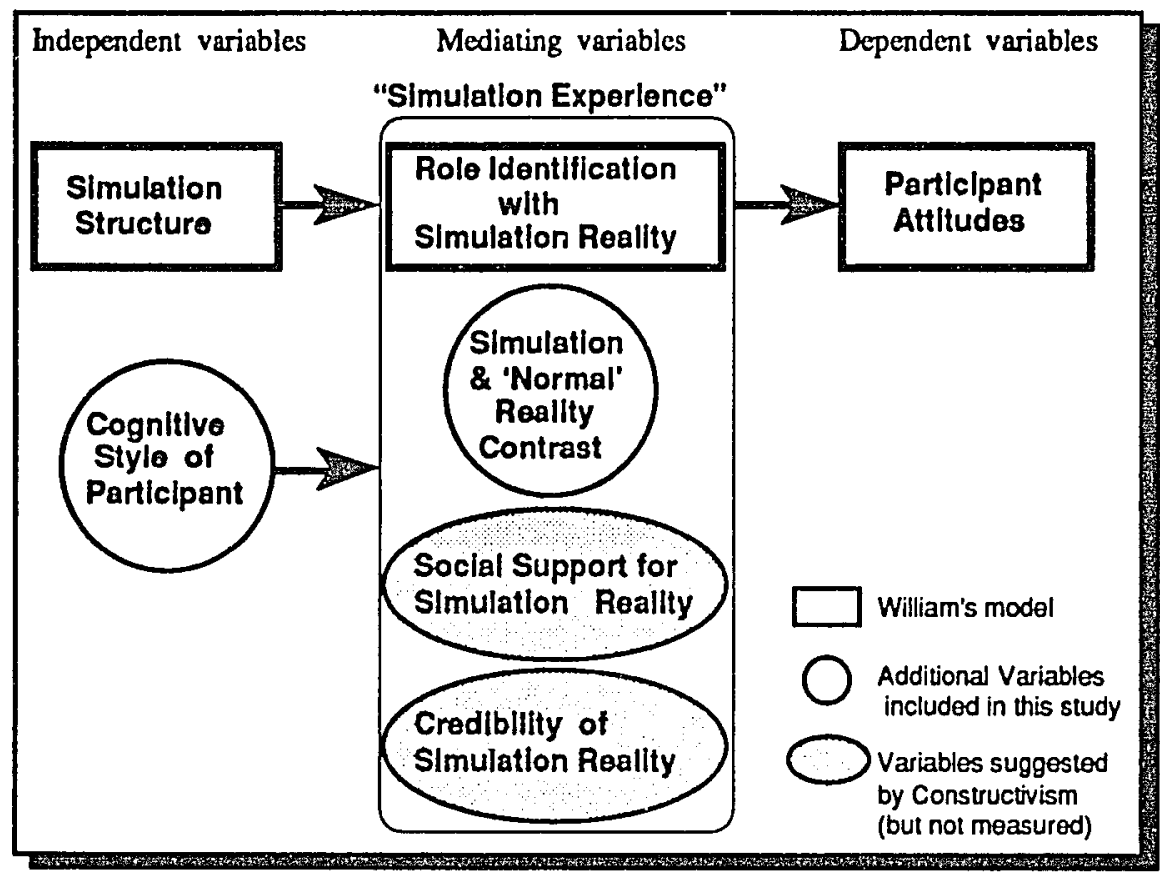

Figure 12. Mediating variables suggested by Constructivism.

Later, in attempting to operationalize the "role identification" variable, a second mediating variable was recorded that measured perceived contrast between the reality suggested by the simulation and that which subjects considered the 'normal' organizational reality. Also, the cognitive style of participants was assessed to test the influence of that independent variable on role identification. The particular measure of cognitive style selected was suggested by Bennett's empathy model. (These additional variables are indicated by circles.)

Two additional dimensions (ovals in Figure 12) likely to influence simulation-induced attitude change are suggested by the Constructivist perspective, the credibility of a reality experienced in a simulation and social support for that reality. These were not measured in the present study because they were expected to be constant across treatment groups. 


\section{RELEVANT ATTITUDE CHANGE LITERATURE}

The attitude change literature is relevant to the present research because attitudes constitute the study's dependent variables. In contrast to the rarity of causal models presented in the simulation literature, the social psychology literature on attitudes contains a copious supply. With the exception of Williams, simulation researchers have not attempted to incorporate this attitude research, though as noted they have eschewed explicit discussion of causal models. Basically these literatures exist in isolation as the simulation research reviewed above is not referenced in the Social Psychology literature on attitude change.

\section{Historical Overview}

Attitudes have been the central topic in social psychology since early in this century. In his review, McGuire (1985:236) describes three peaks in attitude research, each with a different focus. The first peak in the 1920s and 30 s was focused on attitude measurement. During the second peak in the 1950 s and 60s attitude change became the dominant topic. The emergence of a third peak in the 1980s and 90 s is expected to focus on "...the content, structure and functioning of attitude complexes and using the systems style of research...". McGuire estimates that more than 1000 articles and books are published each year on the topic - a single year's output is thus larger that the past 30 years of published research on gaming-simulations. Published reviews regularly document the evolving nature of this field (Chaiken and Stangor 1987, Cooper and Croyle 1984, Dawes and Smith 1985, McGuire 1985) with reviewers listing the research topics that they omitted to make their task manageable. 


\section{Attitude Definition}

Despite more than half a century of research the definition of attitude remains a topic of debate within the field. Dawes and Smith (1985:509) begin their review noting,

...there is little agreement about the definition of attitude and hence what aspects of attitudes are worth measuring. In fact, the typical article on attitude contains a discussion of various classical definitions of attitude, which then concludes with a statement of what the author himself or herself will mean by the term. How cumulative science can survive this Humpty Dumpty operationalism is not entirely clear.

Chaiken and Stangor (1987) frame the disagreements as being whether attitudes should be seen as unitary (and affective) in nature, or a binary construct (affective and cognitive components), or tripartite (affective, cognitive and behavioral components). They note the work by Breckler (1984) suggesting that "attitude dimensionality may vary as a function of domain studied". Beyond this, Edwards (1990) has recently suggested that there are affect-based and cognition-based attitudes in the same domain.

In addition to this debate on dimensionality, McGuire (1985:240) discusses seven other issues of contrast among conceptual definitions of attitudes and concludes, "Useful insights are so needed that each theorist should be allowed any surplus meaning that provides her or him with inspiration." There is broad agreement that: 1) attitudes are learned, 2) attitudes influence behavior 3) attitudes involve evaluation and 4) attitudes are relatively enduring (not a temporary mood). Researchers in areas that are subsets of the broadly defined 'Attitude literature' have reached additional agreements within these narrower topics. For example, reviewers 
of the persuasion literature (O'Keefe 1990:15, Reardon 1990) agree that the presence of subject choice is a feature common to that perspective.

The measurement of individuals' mental representations is difficult. In attempting objective measurement of mental models that remain in the province of individual minds, an "astonishing range of diverse views" (O'Keefe 1990) have been developed under the rubric 'attitudes'. The attitude concept has remained viable in part because of this lattitude and McGuire (1985:241) argues that the research benefits from avoiding too restrictive of a definition.

Clarification of attitudes is sometimes attempted by distinguishing them from other mediating dispositional variables such as knowledge, opinions, beliefs, values, habits, motives, traits, emotions, interests, cognitions, etc. ... The result is often confusion rather than clarification... Distinctions deserve to be made only insofar as they make a difference such that the distinguished variables relate differently to third variables of interest. ...Using attitudes as a contrast term to opinions, beliefs, etc., scores poorly on both these criteria.

\section{A Broader Context for Attitude Research}

Palmerino et al. (1984:179) suggest that a re-conceptualization of 'attitude' is necessary. They want research on attitudes and attitude change to be broadened by the inclusion of context.

What is notably lacking in present day conceptualizations of attitude is reference to ... the context surrounding the attitude. That is, we need not only speak about an attitude with respect to an object, but we need to locate this relationship, between the holder of the attitude and the object, within a specific context. The form of this relationship can be radically different given different contexts. ... To appreciate a contextual formulation one should consider gestalt and field theories.

This is a call for the 'systems style' of research that McGuire predicts and is consistent with the above mentioned recommendation by Morey and Luthans $(1984,1985)$ for studying organizations. The emphasis on context 
leads Palmerino et al. to conclude that attitude measurement in laboratory or classroom settings could be quite different from measurements made in other contexts. This supports the decision in the present study to measure attitudes in a field setting.

Palmerino et al. (1984:183) focus on one of the factors that determines context, the extent that subjects employ 'mindfulness' or 'mindlessness' information processing strategies. These are similar to Hofstadter's (1979) 'intelligent' and 'machine' modes. Of interest, their description of mindfulness approaches the Constructivist perspective of placing reality construction in the individual.

Mindlessness may be defined as a state of reduced cognitive activity in which the individual processes cues from the environment in a relatively automatic manner without paying attention to potentially novel aspects of those cues. Mindfulness, on the other hand, is a state in which the individual consciously processes environmental cues. Here the person is engaged in actively constructing his or her environment, forming new categories and drawing novel distinctions.

In considering how mindfulness-mindlessness effects context and attitude change they offer the categorization of theories in Figure 13. They conclude that "provoking mindfulness will often lead to attitude change" and suggest that that is accomplished, in general, by actively engaging the subject to create new categories for experience. This is the essence of step five in Bennett's empathy model and the design of the simulation in this study followed the mindful strategies they offer for inducing attitude change.

Discussion of the context for attitudes leads to one of the perennial controversies in this literature, the extent to which attitudes are organized into complex structures. McGuire (1985) distinguishes theories about 'complex ideological systems' and those addressing 'miniature knowledge 
structures'. He lists terms from the latter literature including schemata, frames of reference, implicit physics, implicit personality, scripts, modes, and frames to describe these systems of attitudes. The perspective of this study, focusing on the system-level constructs of simulation role and the frame-ofreference-shifting model of change, shares with the schemata research a perspective of individual's having structured cognitive systems.

\begin{tabular}{|ll|}
\hline \multicolumn{1}{|c|}{ Primarily Mindful } & \multicolumn{1}{c|}{ Primarily Mindless } \\
\hline & Attitude Theories \\
Dissonance theory & Classical conditioning theories \\
Balance theory & Operant conditioning theories \\
Expectancy-value theories & \\
Attribution theories & \\
Social judgement theory & \\
Strategiess for Inducing Attitude Change & \\
Implicit conclusions & Explicit conclusions \\
Two-sided arguments & One-sided arguments \\
Active audience role & Passive audience role \\
Innoculation-forewarning & Repetition \\
& Communicator effects \\
& Distraction effects \\
\end{tabular}

Figure 13. Categorization of attitude theories by Palmerino et al.

\section{Schema Theories}

Taylor and Crocker (1978:91) review the work of more than 150 researchers and ask if this work on the schema concept meets the criteria for a theory. Their answer is a qualified 'yes'. They offer the following definition of schema which is consistent with 'frame of reference' as used in this study. ...a cognitive structure that consists in part of the representation of some defined stimulus domain. The schema contains general 
knowledge about that domain, including specification of the relationships among its attributes, ...A schema can be thought of as a pyramidal structure, hierarchically organized with more abstract or general information at the top and categories of more specific information nested within the general categories. ...The schema is connected to other schemas through a rich web of associations, particularly at the lower levels of greater specificity.

These authors find considerable evidence supporting the schema concept and suggest topics that future research needs to explore. Chief among these is improved conceptual clarity and "methods of measuring schemas independent of their processing functions". Experimental designs have typically assessed how subjects judge, recall or classify information to measure the effects of schematic processing, rather than attempting a direct assessment of schemas. This literature adopts an information processing perspective and views schematic content as highly cognitive. Hence they are more likely to speak of 'belief change' than 'attitude change'.

The ontological assumptions of these researchers places them in the center of Figure 9 (page 33). They note "schemas do distort reality" and seek to understand when and how this occurs rather than making the Constructivist assumption that schemas are the individual's reality. Considerable research effort has been invested in exploring the consequences of using the 'wrong' schema and patterns of errors that result from schematic processing (Kahneman, Slovic and Tversky, 1982). This research is a major source of empirical support for the existence of schemas.

Questions about how schemas are created or changed received little discussion in Taylor and Crocker's review and they consider this an important topic for subsequent work. More recently Crocker, Fiske and Taylor (1984) discuss three models for schema change, the bookkeeping model, the conversion model and the subtyping model. The conversion model 
is one of extensive change in a sort time in response to a particular experience. It most closely matches the system-level model proposed in the present study.

Weber and Crocker (1983) sought to compare these three models and reported support for the subtyping model though whether this result is applicable to other conditions is open to question. Crocker et al. (1984:220) comment on the schema measurement problem though they offer no solution.

The schema literature provides a theoretically rich basis for predicting when schemas will and will not change, how they change, what features are likely to change, and what kind of data will prompt change. It is therefore somewhat surprising that little empirical work has actually examined these hypotheses. No doubt one problem that faces the researcher potentially interested in addressing schema change is the problem of how to measure a schema.

As these authors observe, much of the schema literature is theoretical, and the empirical work has not produced a widely accepted approach to measure schemas ${ }^{12}$. Recent work has reviewed (Walsh, 1990) and demonstrated (Walsh and Charalambides, 1990) approaches to measuring schemas. While the level of analysis adopted in the schema research is consistent with simulation roles and the frame-of-reference change model, the primary focus in the present study was attitude change as in previous simulation research.

The schema literature indicates that some of those investigating change in mental models consider fertile a systems approach that addresses

12 It should be noted that Taylor and Crocker express concern about attitude-schema compatibility because they find no need for an affective component in the hypothesized models. In addition, attitudes have often been measured along a 'good-bad' continuum while they believe schematic content may not necessarily be organized along polar dimensions. Others (e.g., Walsh 1990) are less concerned about these issues. 
knowledge structures and a context broader than single attitudes.

Substantial problems in measuring these mental systems remain, but as the research on schema change garners support, it represents a change in how mental models as dependent variables are conceptualized.

There is less evidence of a change toward a systems approach in thinking about the independent variables. Questions about what combinations of information delivered though what types and sequences of media to people with which characteristics remain beyond those asked by research in attitude change or the more narrowly defined schema research. Research on how simulations cause changes in subjects' mental models perhaps remains the most advanced work on system-level interventions.

\section{Centrality of the Dependent Variables}

Determining the centrality of attitudes is difficult, especially when subjects are not studied in depth or they are drawn from a heterogeneous population (such as students). Concern that the attitudes measured in the present study be well integrated in subjects' schemas led to the selection of a field rather than a classroom setting. While no claim is made that the attitudes measured in this study are the 'most central' for subjects, the investment in ethnographic activities in Phase 1 of this study supports the argument that they were not peripheral.

The centrality within subjects' cognitive structures of the attitudes expected to change is an issue in research on attitude change. Researchers accept that attitudes are resistant to change in part because they are embedded in a system of other attitudes and beliefs (Bem 1970). What can be labeled 'systemic resilience' is apparent in the advice to managers that Bowditch and Buono (1985:96) offer: 
Individual values and attitudes, especially those that are deeply held, are notoriously difficult to change directly because people's values tend to be part of an interrelated system in which each value is tied to and reinforced by other values. Thus, managers must realize that it is virtually impossible to change a particular value in isolation from an individual's other values.

The more central an attitude is in an individual's cognitive structure, the more resilient that attitude because of its support from related cognitive elements (Bem 1970, Kelly 1955). For example, a change in economists' attitudes about free trade would be more a substantial demonstration than, say, a change in college freshmens' attitudes about a hypothetical 'Lord South" of whom they had never heard prior to playing a simulation of his life. Without some assessment of the centrality of the attitudes targeted for change for the subjects, a claim that attitude change occurred during an intervention is difficult to evaluate.

As Palmerino et al. (1984) noted, most of the research on attitude change does not attempt to describe the broader context of subjects and their attitudes. Systemic resiliency and heterogeneity of subjects' cognitive models may therefore be undetected confounding factors that complicate the comparison of results from attitude change studies.

\section{The Measurement of Attitudes}

Given the great range of definitions of attitudes employed by researchers it is not surprising that a variety of methods have evolved to measure them. Attitude assessment remains a substantial area of research and Dawes and Smith (1985) provide a recent review of that work. Direct techniques, in which subjects are simply asked to respond to statements about an attitude object, are easy to construct but may be unreliable. Quasi-direct procedures, such as the Likert and Thurstone scales, require significantly more effort to 
construct but yield more reliable instruments. These latter procedures involve collecting numerous attitude-relevant statements and filtering these to produce the final instrument. They differ in how that filtering is accomplished, though they both include evaluating responses to the statements by test groups. 
CHAPTER III

\section{RESEARCH QUESTIONS AND HYPOTHESES}

\section{DEFINITION OF VARIABLES}

This study was designed to explore the relationships that underlie the causal model relating simulation to attitude change presented in Figure 6. These include whether simulation structure or cognitive style influence role identification and whether identification influences attitude change. There are seven research questions in this study that explore parts of that model. These are labeled R1 through R7 and diagramed in Figure 14. Three treatment groups represented the different values for the independent variable 'Simulation Structure' in Figure 14. These and a Control group are briefly described in Figure 15 and in greater detail in the methodology section (Figure 29).

The independent variable 'Cognitive Style' was defined using a subset of the Davis (1983) Interpersonal Reactivity Index. Two seven-item subscales, labeled "Perspective Taking" and "Fantasy" by Davis, were included in the pre-simulation questionnaire. These are the cognitive dimensions in the IRI

and separate scores for the two subscales were calculated to allow the testing of hypotheses with "Perspective Taking" and "Fantasy" as measures of the independent variable 'Cognitive Style'.

Participant 'Role Identification' was measured to operationalize the broader concept of 'Simulation Experience' - how subjects differ in their 
experience of the simulation. It was measured by having the simulation software present subjects with questions during the simulation class. The questions are listed and discussed in the Results section under Research Question 3 (Figure 36). Responses to these questions function as dependent variables for testing hypotheses in Research Questions R3 and R4, and function as independent variables in Research Question R5.

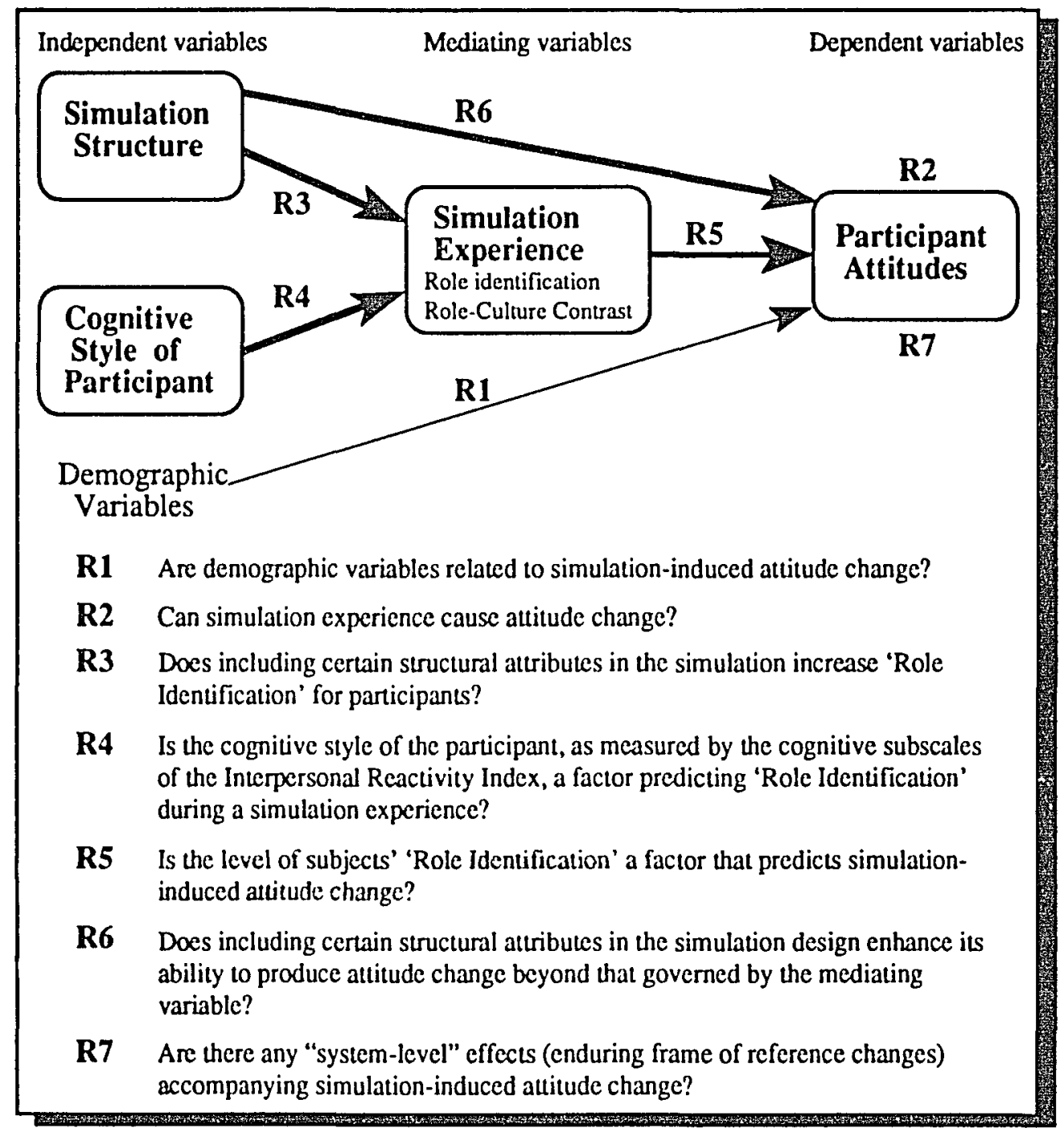

Figure 14, Study variables (boxes) and research questions (arrows). 
'Participant Attitudes' were was assessed by comparing pre-simulation and post-simulation questionnaires. A global "Quality Growth" variable was created by combining all forty questionnaire items that were measured for change. In addition, subsets of these questionnaire items were grouped into eight 'subscale' variables as discussed in the Methodology section.

\begin{tabular}{|ll|}
\hline \multicolumn{1}{|c|}{ Group } & \multicolumn{1}{c|}{ Attributes } \\
\hline Enhanced Simulation & (Enhanced Simulation and Video) \\
Video Control & (Enhanced Simulation, no Video) \\
Simplified Simulation & ("Enhanced" simulation features omitted; \\
Control Group & (Nideo included) \\
\hline
\end{tabular}

Figure 15. Treatment group nomenclature.

\section{STATEMENT OF RESEARCH QUESTIONS}

\section{Research Question 1}

Are demographic variables related to simulation-induced attitude change?

The causal model explored in the present study does not directly suggest hypotheses relating demographic variables to study variables. Still, tests were made for relationships between attitude change and subject sex, age and length of employment in the client organization. Because the simulation classes were conducted in two week-long sessions with different facilitators, a 'Simulation Delivery' variable indicating which session the subject had attended, was also tested.

A question of the form 'Is there a significant difference in participants' attitude change attributable to (demographic variable $\mathrm{x}$ )?' was tested for each 
combination of demographic variable and attitude measurement (dependent variable).

\section{Research Question 2}

Can simulation experience cause attitude change?

Numerous reports in the literature indicate that simulations have caused attitude change. The present concern addresses the more restricted question of whether the WINNING AT DESIGN AUTOMATION simulation caused attitude change in the population and experimental conditions of this study. For each treatment group the hypothesis is stated:

$\mathrm{H} 1$ : Is the post-simulation attitude score significantly greater than the pre-simulation score for (dependent variable $\mathrm{X}$ )?

Each of the dependent variables generates a separate hypothesis. Because the expected direction for change on each dependent variable is specified, a one-tailed test is appropriate.

Research Question 3

Decertain structural attributes in the simulation increase 'Role Identification' for participants?

This research question compares subjects in the different treatment groups on their scores for the mediating variable 'Role Identification'. Because the direction of difference is specified, a one-tailed test is appropriate.

H2: Do subjects in the Enhanced Simulation group report greater Role Identification scores than those in the Simplified Simulation? 
It is expected that there will be no difference between the Enhanced Simulation and the Video Control group for the mediating variable. A twotailed test is appropriate for this hypothesis.

H3: Is there a significant difference between the Role Identification scores of subjects in the Enhanced Simulation group and those in the Video Control group?

\section{Research Question 4}

Is the cognitive style of the participant, as measured by the cognitive subscales of the Interpersonal Reactivity Index, a factor predicting 'Role Identification' during a simulation experience?

Two measures of cognitive style were calculated from the IRI questionnaire items, "Perspective Taking" and "Fantasy". Each of these is paired with the mediating variable ('Role Identification' acting as a dependent variable). The direction of the relationship has been predicted and therefore one-tailed tests are appropriate. The hypotheses are:

H4: Do subjects who score higher on "Perspective Taking" report greater scores on 'Role Identification'?

H5: Do subjects who score higher on "Fantasy" report greater scores on 'Role Identification'?

\section{Research Question 5}

Is the level of subjects' 'Role Identification' a factor that predicts simulation-induced attitude change?

The causal model hypothesized in this study predicts that greater 'Role Identification' will cause greater attitude change. Because the direction for the relationship has been predicted, a one-tailed test is appropriate. 
Separate hypotheses involving the different measures of attitudes will have the form:

H6: Do subjects who report greater levels of 'Role Identification' have greater attitude change (dependent variable $\mathrm{X}$ )?

\section{Research Question 6}

Does including certain structural attributes in the simulation design enhance its ability to produce attitude change beyond that governed by the mediating variable?

This question seeks to determine if attitude change is influenced by the simulation structure beyond any influence of the mediating variable. Comparison of the magnitude of change for the Simplified and Enhanced Simulation groups for each dependent variable will provide evidence whether the Enhanced Simulation version is more effective than the Simplified. The form of the hypotheses is:

H7: Is the change for (dependent variable X) in the Enhanced Simulation group significantly greater than the change for the Simplified Simulation?

\section{Research Question 7}

Are there any "system-level" effects (enduring frame of reference changes) accompanying simulation-induced attitude change?

This question concerns whether experiencing the simulation led to lasting change at a level of analysis greater than that of a single attitude, for example something that might be labeled simulation role, schema or frame of reference. Four indicators of whether subjects adopted a more global "system-level" change were considered: 
1) whether attitude change occurred in the capstone variable of the frame of reference,

2) whether attitude change occurred uniformly for all variables in the frame of reference,

3) whether correlations between the dependent variables changed, and

4) the shape of the distribution of attitude change. 
CHAPTER IV

RESEARCH DESIGN AND METHODOLOGY

The discussion of methodology has five sections: a research design overview and a section corresponding to each of the four phases of the study shown in Figure 16.

1. Qualitative Data Collection.

2. Frame of Reference \& Questionnaire Design.

3. Simulation Class Design \& Production.

4. Quantitative Data Collection.

Figure 16. Study phases.

\section{RESEARCH DESIGN OVERVIEW}

A questionnaire and simulation class were developed from material gathered in Phase 1 of the project. Two months before the first class, the presimulation questionnaire and cover letters (Appendix D) were sent to a random sample of employees along with a stamped envelope addressed to the researcher. It included a subset of the Davis (1980) empathy instrument to measure cognitive style. Scores on this instrument were used in the assignment of subjects to groups to produce a blocked design. Three different versions of the simulation class were used to produce three treatment groups. 
There was also a control group with no simulation experience. Subjects assigned to the treatment groups were invited to attend the appropriate simulation class which was conducted by the client organization. Data for the mediating variables, 'role identification' and 'Role-Culture Contrast', was collected by the simulation software during each class. The same questionnaire was again sent to subjects fifteen days after their simulation class.

\section{QUALITATIVE DATA COLLECTION — PHASE 1}

Go to the practical people and learn from them; then synthesize their experience into principles and theories; and then return to the practical people and call upon them to put these principles and methods into practice so as to solve their problems and achieve freedom and happiness.

- Chinese saying

The primary goal of Phase 1 was to locate issues within the culture of the client organization suitable for the simulation. Several requirements of the client organization helped determine these issues.

\section{Issue Selection Criteria}

The project's sponsor indicated a strong preference for the issues in the simulation to apply to as many employees as possible. For example, issues relevant only to employees in the sales group would have had too limited an appeal. A broad appeal would make it easier to get a sufficient number of subjects, a problem that became greater than the original agreement with the client had indicated.

For research design purposes, suitable issues had to address some potential change. This implied defining a gap between the actual situation 
and an ideal one. The direction for change indicated by the simulation had to be consistent with management's views.

Initially the researcher looked for issues concerning the organization's external environment. The organization's competitive or technological issues were expected to be changing rapidly and therefore appropriate for the redefinition that could be presented in a simulation. Although employees were candid during interviews, it became obvious that to include such issues in the simulation, and to produce a public document describing them could encounter confidentiality problems. Instead, the decision was made to address internal issues only.

Interviews were conducted for five months before a tentative frame of reference, the 'Mentor Graphics Decision Making Style', (MGDMS) was offered to the project sponsor. MGDMS included attributes such as an 'open door' policy, focus on winning, encouragement of risk taking, and 'going the extra mile' for customers. It met with little enthusiasm. The sponsor indicated that, while this described Mentor Graphics, other excellent companies shared many of these same attributes. He stated his hope for something more distinctive to Mentor Graphics, and encouraged the researcher to continue the interviews. After three additional months of interviews, diagrams presenting three new frames of reference, 'Quality Growth', 'Coaching Winners' and 'Building Partnerships' were approved by the CEO, president and project sponsor as being significant issues and as setting out positions that the company would support. These frames of reference became the basis for the questionnaire, video tape and simulation. 


\section{Coyer Story}

One of the study's goals was to obtain responses from the participants that were as honest and unbiased as possible. In much previous simulation research, it was possible for the desired responses to be suggested to participants by a close association between the simulation experience and the measurement of the dependent variables. To avoid this, the simulation classes were separated in time and context from the other dissertation activities.

The researcher was expected to become well known in the organization over the course of the project. A front-page story and photo in the company's monthly newsletter announced the project and invited employees to arrange an interview with the researcher. A cover story maintained that the student was collecting information on corporate culture for his doctoral dissertation. That is, the early phases of the project were revealed entirely. Interviewing, participant observation, video tape production and questionnaire distribution were all conducted under the umbrella of this story.

Only a half-dozen employees knew about the creation and testing of the simulation. The simulation classes were announced and conducted by the Human Resources Department as part of normal company training, and the principal investigator was not present. Post-simulation interviews indicated that the cover story was effective and that subjects did not associate the simulation class with the questionnaires.

\section{Primary Qualitative Data Source - Interviews}

Four sources of data were used to learn about the culture and to determine issues for the simulation. These were personal interviews, participant observation, printed material about the organization and 
responses to the questionnaire. Each of these is discussed separately in this section. Two other minor sources, interactions during the video tape production and comments recorded during the simulation classes, also helped the researcher assess the culture of Mentor Graphics, and are documented in the discussions of those topics.

The largest source of data was personal interviews ${ }^{13}$. Over 200 formal interviews, ${ }^{14}$ with more than 125 people, were conducted between January 1987 and May 1988. These included interviews during four days in June 1987 in San Jose, the second largest Mentor Graphics site. Typically, interviews were conducted in the employees' offices and lasted 45 to 90 minutes.

An initial strategy was to select people who worked in engineering functions in order to understand the organization's core technology. This provided an awareness of product lines, technical terminology and market segments, and proved to be extremely valuable in subsequent interviews. Greater emphasis was later directed at other functions in the organization to ensure a more balanced sample. People to be interviewed were selected from names mentioned in previous interviews. A name of someone to interview was occasionally selected at random from the company phone list.

13 Because of the travel time and cost involved in visiting international sites and the North American sales offices, these groups were substantially under-represented in the study. Further, it is likely that substantial differences exist between the cultures of these groups and the primary population in Beaverton and San Jose. Discussion of and claims about "the Mentor Graphics culture" should be taken as exclusive of these groups.

14 The term "formal interview" is used to indicate that the interview was scheduled in advance, allowing informants time to prepare their thoughts. Written notes were almost always made during formal interviews. The term is contrasted with "informal interviews", which were spontaneous and usually included no note taking. 
As the months went by and the principal investigator met more and more people, he would often talk to someone in the hallway without having arranged an interview. Written records were not made for many of these informal interviews. The number of such meetings was greater than the 'formal' interviews reported above. In contrast to the formal interviews, these informal discussions came later in the project.

Only three persons refused to be interviewed. Most people interviewed were enthusiastic about having an opportunity to talk about the culture. Several sought out the researcher and arranged an interview to tell a story or make a comment.

Informants were advised of the nature of the study as revealed in the cover story, and were assured that their comments and any interview notes would remain confidential. They were also told that their identities would not be disclosed if any quotes were used. Many of those interviewed expressed no concern that their views might be made public and some even asked that their comments be repeated to senior managers, adding, "Tell him I said so". The atmosphere throughout the organization was very open during the interviews.

The format of the interviews followed the suggestions of Spradley (1979). The intent was to elicit description of the informant's social reality in the language he or she used in everyday organizational interaction. A list of terms collected during the interviews is presented in Appendix B. These would be described as 'cover terms for domains' by an ethnographer.

Statements taken from notes made during each interview became the basis for the items in the questionnaire. About 40 of the interviews were recorded on audio tape. 
Informant Problems. ${ }^{15}$ Many employees at Mentor Graphics expressed interest in the project and were sincere in wanting to help the researcher. Unfortunately for several reasons they were not particularly good informants. First, they are highly educated people whose training has developed their analytical skills. Many wanted to analyze their view of the culture, thus side stepping the ethnographer who was trying to collect descriptive statements, not analytical reflection.

Second, the people at Mentor Graphics are very bright. The ethnographer's approach of listening to descriptions of events and then reviewing those descriptions to repeatedly ask hypothetical questions quickly bores them. Consider Spradley's (1979:28) description of the differences between ethnographic interviews and the normal conversations between friends:

"A friend does not ask the same question over and over; an ethnographer does. A friend does not ask for endless clarification; an ethnographer does."

After a few statements about a topic, informants wanted to move on. "We already talked about that... what other topics should we discuss?" was a frequently heard response.

Another problem that interrupted the typical ethnographic approach to data collection was that many of the informants were interviewed only once. This was dictated by their very busy schedules and the type of contribution they wanted to make to the project. Many had a story to tell or wanted to

15 In Ethnoscience, persons interviewed are referred to as "Informants". Spradley (1979, pp 29-32), in keeping with the Constructivists' careful attention to language subtleties, clearly distinguishes the different nature of the relationship between researchers and "Informants", "Respondents" and "Subjects". 
talk about some issue. Once they had done so, their interest in further discussion was low. A related issue was the breadth of cultural knowledge covered by the interviews. Engineers talked about engineering problems, sales people about customers, lawyers about contracts and people in shipping about testing Apollo nodes. This narrowness of content, coupled with the relatively few repeat interviews, meant that only a fraction of each interview related to the cultural themes and issues that later became the focus of the simulation.

\section{Other Qualitative Data Sources}

Participant Observation. The methods used in these activities are described by Spradley (1980). A common opportunity for participant observation at Mentor Graphics was the 'mixers', the term used at the company to refer to a large-scale, informal social event. Mixers might be called a 'party' in other groups. Food was served and often there was live music and an activity such as, balloon toss, team volleyball, dancing, or skiing. About one mixer a month was held during the 15 months of intensive interviewing. These were typically held on a Thursday or Friday afternoon beginning at 3:00 or 4:00 pm and lasting three to six hours. These were an excellent way for the principal investigator to meet many employees and frequently such meetings lead to interviews.

In addition to the mixers, the following events were included in the participant observation: end-of-quarter update meetings; departmental strategic off-site meetings; Mentor Users Group (MUG) annual meetings; customer demos; benchmarks; staff meetings; employee birthday parties; annual sales kickoff; trade shows and conferences; Mentor Graphics annual suppliers conference; annual corporate strategic off-site; technical and 
managerial training classes. These are all cultural scenes at Mentor Graphics. The principal investigator attended each of these events at least twice.

A number of these events included people who were not employees of Mentor Graphics such as suppliers, customers, and competitors. Talking with these people and watching them interact with Mentor Graphics employees was helpful in confirming impressions from the interviews and in seeing how outsiders viewed Mentor Graphics.

Printed Material. A substantial body of printed material was collected and reviewed throughout the project. Annual reports were an obvious starting point, as was the company newspaper, "Voices", which is printed monthly and contains many articles written by senior management. In addition the company distributes "Visions" a quarterly newsletter for customers. Product brochures and advertisements, the new employee folder, the 'Mentor Graphics Story' (a 25 page story describing the early days of the company), materials from internal training classes, slides prepared for public presentations, articles about Mentor Graphics in the trade press and a wide variety of internal memos completed the list of printed materials. Mentor Graphics is an organization that conducts a great deal of its internal business informally. Printed materials were still a useful source of information about the organization.

Questionnaires. The primary purpose of the questionnaires was to collect the quantitative data to be used in the hypotheses testing phase of this study. In addition, some of the questionnaire data supplements the above sources in describing the culture. This data was collected after most of the interviews had been completed. 


\section{QUESTIONNAIRE CONSTRUCTION — PHASE 2}

The questionnaire co-evolved with the three frames of reference during Phase 2 of the project. The interviews had captured hundreds of attitude statements by members of the Mentor Graphics culture. Those which fit into the frames of reference were used unaltered in the questionnaire. These statements comprised about 75 percent of the initial questionnaire. The remaining questionnaire items were created by the principal investigator. These latter items still used the informants' language, but addressed relationships that had not been expressed during the interviews.

Three issues served as the starting point for building the frames of reference. These were: 1 ) a wide spread concern that the company was becoming more bureaucratic, 2) an expressed need by employees for more coaching and 3) the stated management desire for more "partnerships". (See discussion in Appendix C.) The content and structure of these frames of reference evolved gradually over a period of about two months as the principal investigator iterated between modifying the frames of reference, assessing their internal logic, and reviewing the interview statements for their support for or conflict with the frames of reference.

A draft of the questionnaire was given to the dissertation committee members and five employees of Mentor Graphics. Their feedback was incorporated into the pilot questionnaire.

Pilot Questionnaire and Reduction to Pre-Simulation Questionnaire

In May of 1988158 pilot questionnaires were sent out to managers of Mentor Graphics. A short cover memo from the CEO asked employees to comply. A separate memo from the researcher assured anonymity and 
provided instructions. Eventually 134 completed questionnaires (85\%) were returned. This response rate was outstanding given the length of the questionnaire (219 total items) and the very busy schedules of the employees. It was originally intended that data from this pilot questionnaire would provide the pre-simulation measurement. However the extended delays in making the video tape and scheduling the simulation classes, as well as the need to include more employees, lead to the distribution of a second presimulation questionnaire.

The second (pre-simulation) questionnaire was about half the size (94 items) of the pilot questionnaire for several reasons. First, it followed the completion of the simulation. The 'Coaching Winners' and 'Building Partnerships' frames of reference were de-emphasized during the development of the simulation to reduce its complexity and playing time. Many questionnaire items were dropped because after this refinement in focus, they were not addressed by the simulation.

In addition, examination of the pilot questionnaire data indicated that some questionnaire items had mean scores near the end of the scale, thereby making it difficult to demonstrate change. Several items were dropped for this reason. Finally the responses to the Rokeach Dogmatism scale on the pilot questionnaire had been so aberrant that this instrument was dropped from the second questionnaire. This left only the Davis Empathy scale as a measure of cognitive style. Appendix $D$ includes a copy of the questionnaire.

\section{THE “QUALITY GROWTH” FKAME OF REFERENCE}

One anomaly in the MGC culture was the attitudes surrounding bureaucracy. In all other domains respondents indicated confidence that they 
as individuals and Mentor Graphics as an organization would overcome considerable obstacles. However, they repeatedly commented that the "small company atmospheren which they enjoyed was slipping away as the company grew. They also stated that MGC was becoming more bureaucratic, a condition which they considered very unfavorable. Employees expressed a uniform view that there was little opportunity to avert these trends. They were resigned to an increasingly unpleasant future regarding the negative influence of organizational growth on the culture ${ }^{16}$.

A primary attribute of a "small company atmosphere" is that most of the individuals in an organization have an internal locus of control. They see themselves as being "in control of our own destiny". The definition of "bureaucracy" used at MGC had at its core an external locus of control. Respondents stated than in a bureaucracy people often felt like "cogs in a machine". During the interviews no respondent ever stated that a "small company atmosphere" was the opposite of a "bureaucracy", indeed fow talked about both concepts. But stripped to this abstract core these concepts can be construed as opposite poles of a dimension along which the company's culture was evolving. Guy Kawasaki (1990) has provided an entertaining description of the culture of Apple Computer which had many similarities to the "small company atmosphere" at Mentor Graphics.

CEO Tom Bruggere summarized what many others had indicated, "We like our culture, we don't want it to change. Its an important part of what makes this company successful and a fun place to work." This desire to

16 See Peter Block, The Empewered Manager, (1987) or John Sculley's Odussey (1987) for accounts that describe a similar dimension of organizational culture. 
retain the existing culture and the contrast between the widespread confidence and the external locus of control expressed regarding the changing culture, suggested the theme for a new frame of reference. The idea was to introduce an internal locus of control on the growth-culture issue.

A new frame of reference was created which was intended to be conveyed by the simulation. "Quality Growth" suggested that "small company atmosphere" and "bureaucracy" were mutually exclusive paths for organizational growth and that each individual had opportunities that would maintain the small company atmosphere and thus avoid bureaucracy. The "Quality Growth" frame of reference suggests it is possible to indefinitely thwart the trend toward bureaucracy. ${ }^{17}$

\section{A New Frame of Reference - Quality Growth}

The essence of the 'Quality Growth' frame of reference is represented in Figure 17 which appeared in the Beta cultural role description and was a graph available during the Beta play of the simulation. The " $\mathrm{X}$ " superimposed on this figure marks the starting point of the simulation. Growth along the $\mathrm{X}$-axis is determined by the simulation software and is consistent for all players in all games. Player decisions determine the 'Quality' component on the Y-axis.

Organizational growth in the "Quality Growth" frame of reference is construed as having two dimensions. One is the common quantitative 'Size'

17 Whether it is in fact possible for an organization to avoid "bureaucracy"is coextensive with the definition of "bureaucracy" that one adopts. The attributes of a "small company atmosphere" included in the "Quality Growth" frame of reference make it possible for a very large company also to have this type of culture. The adoption of the "Quality Growth" frame of reference was seen as the empowerment of employees regarding the growth-culture issue. 
measured by number of employees. The second dimension is 'Quality' which is defined in the simulation as a function of the strength of the vision, the strength of partnerships among employees, the level of coaching, the amount of rewards individuals receive, the amount of risk taking and the amount of pushing back in the organization. In short, 'Quality' is here seen as a function of the various actions which simulation participants can take to maintain the small company atmosphere and inhibit bureaucracy. The measure of 'Quality' in the WINNING AT DESIGN AUTOMATION simulation is a function of these six variables.

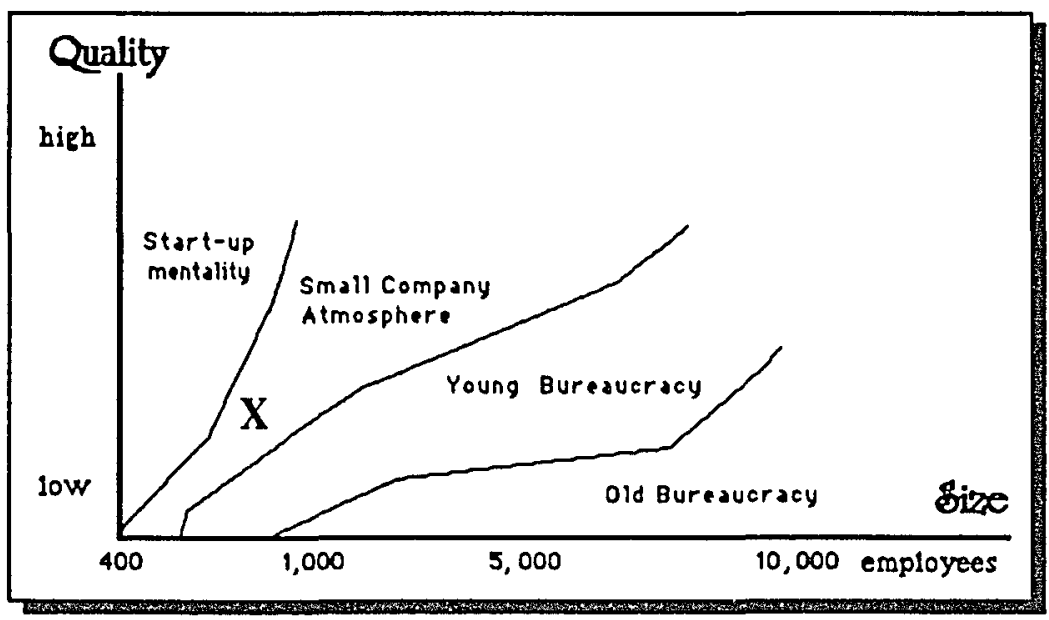

Eigure 17. The Quality Growth graph - the Beta perspective.

As Figure 17 shows, the ratio of size and quality determines the type of culture in a company. The attributes used to describe these concepts in the simulation are consistent with their definition in the Mentor Graphics culture.

Relationshios among the Quality Growth Variables

The Quality Growth frame of reference was designed to have the structure displayed in Figure 18 reflecting the linkages between dependent 
variables $D_{n}$ ). The most concrete questionnaire items - Example Actions that inhibit bureaucracy and the Need to Change the frequency of those same actions (D5 and D6)- are at the bottom of the diagram. There is a specific dependent variable designed to measure aspects of two of those concrete actions, Building Partnerships (D7) and Coaching Winners (D8).

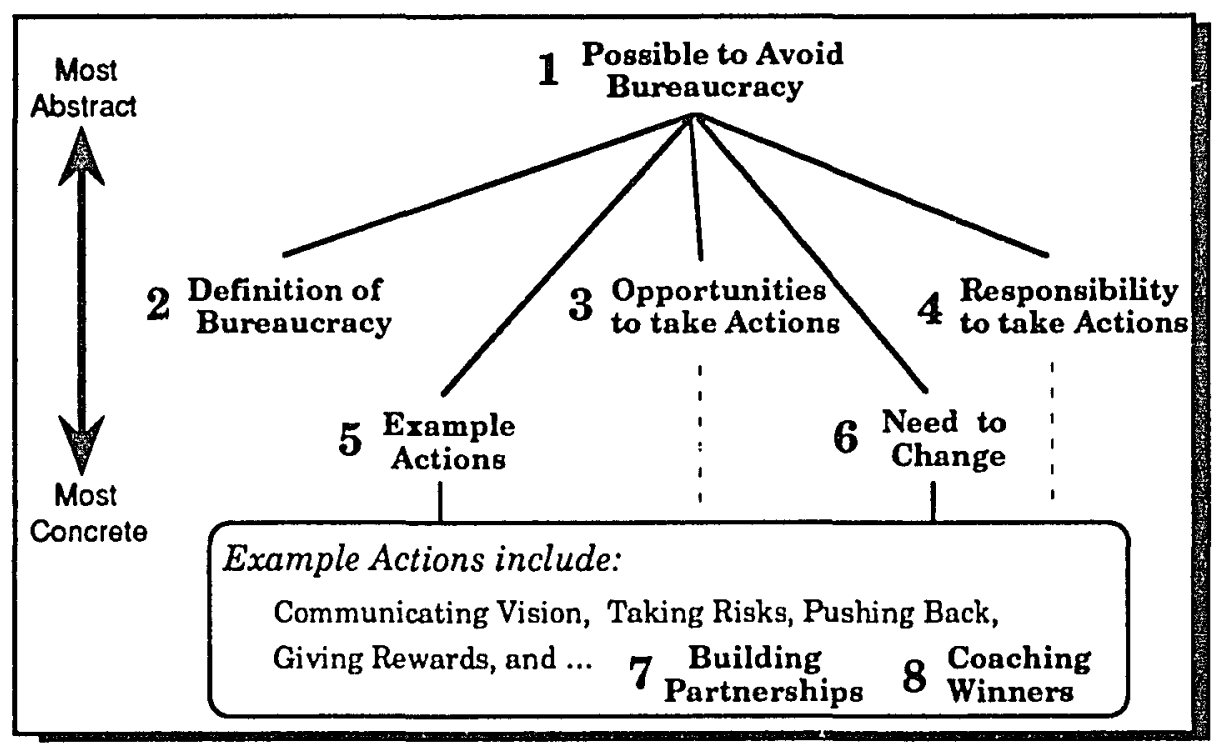

Figure 18. Relationships among variables in quality growth.

At a more abstract level, dependent variable D2 addresses the definitions of "bureaucracy" and "small company atmosphere". Opportunities (D3) and Responsibilities (D4), which were here measured more abstractly than the specific 'Example Actions', are also in the middle of the figure. Variable D3 measures whether participants believe they have opportunities to take the actions to avoid bureaucracy in D5 - Example Actions. Participants' perception of their Responsibilities to take such actions is assessed by $\mathrm{D} 4$. These mid-level variables are in turn less abstract than the capstone variable D1. 
The Beta Cultural Role presented the MGC cultural ideals in the simulation. The logic of this frame of reference is suggestive rather than compelling. Given that certain actions maintain the "small company atmosphere" and thwart "bureaucracy", and also that there are opportunities (and even responsibilities) to take those actions, then it is possible to avoid bureaucracy. The Beta Cultural role suggests, and participants in the simulation experience, that the "small company atmosphere" can be maintained indefinitely.

\section{Measuring Movement Toward "Quality Growth"}

The questionnaire items used to measure elements of the Quality Growth frame of reference were grouped during the design of the frame of reference. These eight groups became the subscale variables analyzed in Chapter V. Five questionnaire items measure the capstone of the Quality Growth frame of reference - whether a company must become bureaucratic as it grows or whether it is possible to maintain the small company atmosphere. These items formed dependent variable D1, Possible to Avoid Bureaucracy, and are displayed in Figure 19.

21. It is possible for Mentor Graphics to become very large while still retaining its "small company" atmosphere.

30. As Mentor Graphics becomes a larger organization it will become more bureaucratic.

35. As organizations grow it is inevitable that they become more bureaucratic.

50. When the growth in an organization has sufficient quality, the organization will not become a bureaucracy.

71. As Mentor Graphics becomes a larger organization it will lose its small company atmosphere. (note: items $30.35 \& 71$ reverse scored) Cronbach's Alpha $=.655$

Figure 19 Possible to Avoid Bureaucracy, dependent variable D1. 
Five questionnaire items were included to measure attributes of bureaucracy or relate it to "small company atmosphere". Together these items were labeled Definition of Bureaucracy and are listed in Figure 20. This variable measures whether the simulation experience has suggested changes in how the subjects relate "small company atmosphere" to "bureaucracy", as well as selected attributes of bureaucracy.

9. A 'small company atmosphere' is nearly the opposite of a bureaucratic culture.

12. One of the characteristics of bureaucratic companies is that employces in those companies take very few risks.

33. People in a bureaucracy often have the feeling that they are just another 'cog in the machine'.

55. An organization feels more bureaucratic to individuals who do not have a vision of how they fit into the future of that organization.

62. When an organization becomes bureaucratic, this almost always implies the loss of the small company atmosphere.

Cronbach's Alpha $=.738$

Figure 20 Definition of Bureaucracy, dependent variable D2.

The four questionnaire items in Figure 21 measure general attitudes about the Opportunities (D3) for employees to avoid bureaucracy. The four items in Figure 22 are related to individuals' Responsibility (D4).

6. There are considerable opportunities for each employee to reduce or inhibit bureaucracy at Mentor Graphics.

45. There is little that most individuals can do to prevent the spread of bureaucracy as an organization becomes large.

57. There are opportunities for each individual at Mentor Graphics to make certain that the growth in the company is quality growth.

58. Each employee at Mentor Graphics has opportunities to push back.

(Note: item 45 is reverse scored.) $\quad$ Cronbach's Alpha $=.710$

Figure 21. Opportunities, dependent variable D3. 
26. A major factor that can lead to an organization becoming bureaucratic is when the people don't bother to work against the growth of bureaucracy.

39. Managers have a special responsibility in achieving quality growth through their coaching of other employees.

48. Mentor Graphics employees have a responsibility to prevent bureaucracy in the company.

95. Managers at Mentor Graphics have a responsibility to communicate a vision of the future to the employees in their group.

Cronbach's Alpha $=.681$

\section{Figure 22 Responsibility, dependent variable D4.}

The seven questionnaire items addressing specific examples of actions

that individuals can take to prevent bureaucracy are shown in Figure 23.

Together these seven items comprise the Example Actions (D5) variable.

10. When individuals make the effort to express their opinions it helps the company avoid bureaucratic growth.

47. When managers at Mentor Graphics coach employees it helps the company to achieve quality growth.

59. Helping others at Mentor Graphics see how they are having an impact on the company will reduce the feeling of being in a bureaucracy.

63. If each person at Mentor Graphics takes more risks, there will be less bureaucracy in the company.

64. Employees at Mentor Graphics can help reduce bureaucracy by forming partnerships with people in other parts of the company.

72. When managers communicate the vision of the company to employees, they help pravent bureaucracy.

78. When employees push back they can inhibit the growth of bureaucracy at Mentor Graphics.

Cronbach's Alpha $=.804$

Figure 23. Example Actions, dependent variable D5.

The six items used to assess attitudes about the Need for Change at

Mentor Graphics are shown in Figure 24. Dependent variable D6 was

designed to measure if subjects concluded that there was a need for change in 
those behaviors that were linked to the avoidance of bureaucracy in the Example Actions dependent variable (D5).

14. Managers at Mentor Graphics need to do a better job of communicating to employees how the employee's tasks fit into a vision of the company's future.

20. As a company grows larger, building partnerships with others in the company becomes more important.

28. In the future we will need to do a better job of forming partnerships at Mentor Graphics.

34. To maintain our small company atmosphere at Mentor Graphics, we will need to put greater efforts into 'pushing back' in the future.

73. There is a need for individuals at Mentor Graphics to take more risks if we wish to ensure that the company's growth will be quality growth.

75. For Mentor Graphics to continue to be a leader in Design Automation we need to improve our performance in coaching our people.

Cronbach's Alpha $=.739$

Figure 24, Need for Change, dependent variable D6.

The cultural roles in the simulation were presented as fictitious and the simulation therefore did not directly suggest a need for change at Mentor Graphics. However it was expected that participants would see the Beta culture - the Quality Growth frame of reference - as an ideal different from Mentor Graphics' culture and therefore draw the conclusion that change was needed at MGC.

Two of the dependent variables represent the vestiges of the "Building Partnerships" and "Coaching Winners" frames of reference. These were created along with the "Quality Growth" frame of reference at the same time that the pilot questionnaire was being developed. Later they were deemphasized during simulation development due to constraints on class length. Only a small portion of the content in those frames of reference was addressed by the simulation. This change, along with the desire to reduce the 
size of the questionnaire, resulted in only a few of the items measuring these frames of reference being carried into the final instrument. "Coaching Winners" and "Building Partnerships" were reduced to actions - with more conceptual complexity than the other actions of D5 - that employees could take to maintain the small company atmosphere and avoid bureaucracy. Figure 25 displays the four questionnaire items used to create a dependent variable labeled Partnership (D7). There are five questionnaire items in the dependent variable Coaching and these are shown in Figure 26.

22. The essence of a partnership is for each person to respect the viewpoint of the other.

52. The term "partnership" is sometimes used at Mentor Graphics to refer to relationships between people within the company.

56. It is important to take the time to build partnerships with others at Mentor Graphics.

65. Each member in a partnership has a responsibility to express their viewpoint to the other members of the partnership.

Cronbach's Alpha $=.619$

Figure 25. Partnership, dependent variable D7.

18. The key to coaching is to give employees a sense that they are in control of their own destiny.

36. People feel like they have less control over their destiny when they have no vision of how they fit into the future.

60. Employees who feel they have control of their own destiny are more likely to be top performers.

69. One important part of coaching is to convey to the coached person how their tasks fit into the vision of the company's future.

70. When a person is successful in 'pushing back' and can see the impact they have had on the organization, they feel more in control of their own destiny.

Cronbach's Alpha $=.735$

Figure 26. Coaching, dependent variable D8. 


\section{DESIGN OF THE SIMULATION CLASS - PHASE 3}

The primary class activity was playing the WADA simulation and the class goals were to have participants experience and identify with the cultural roles and experience the outcome associated with each role interacting with the simulation model. The Beta role presented the Quality Growth frame of reference while the Alpha role portrayed a frame of reference similar to the existing MGC culture. The class incorporated a number of features to support identification with the cultural roles.

\section{Simulation Class Features for the Alpha Game}

This discussion of features follows the order of class events as shown in Figure 27. It describes the Enhanced Simulation class with differences between treatment groups identified at the end of the section.

Pre-class Preparation. Participants were sent the WADA rules booklet (Appendix E) several days prior to the class. A note indicated that there would be a busy class schedule with little class time to review the rules. The rule booklet does provide clues about the relationships in the simulation model that the class is intended to convey and it was hoped that participants would take the time to read this before the class.

'Ice breaker'. The first activity in each class was an exercise to insure that all participants knew the others in their class. This exercise was also to set the tone of the class which included: having fun, exploring the question "what is our culture at Mentor Graphics and what should it be?", collecting feedback from the participants about the culture and asking whether this simulation class should be offered to other employees. In addition, the ice 
breaker allowed for any late arrivals to occur prior to the beginning of the simulation.

\begin{tabular}{|c|c|c|}
\hline stant & duration & activity \\
\hline 8:am & $20 \mathrm{~min}$ & icebreaker exercise \\
\hline $8: 20$ & $40 \mathrm{~min}$ & $\begin{array}{l}\text { introduction to game } \\
\text { (read Alpha culture, discuss in teams; walk through) }\end{array}$ \\
\hline 9:00 & $90 \mathrm{~min}$ & Play the Alpha game \\
\hline $10: 30$ & $15 \mathrm{~min}$ & break \\
\hline $10: 45$ & $30 \mathrm{~min}$ & debrief Alpha game \\
\hline $11: 15$ & $30 \mathrm{~min}$ & discuss WADA model (overhead slide presentation.) \\
\hline $11: 45$ & $30 \mathrm{~min}$ & read Beta culture (discuss in teams) \\
\hline $12: 15$ & $60 \mathrm{~min}$ & $\begin{array}{l}\text { lunch / watch } 4 \text { video segments } \\
\text { (Stedman, Kiernan, Langhorst, Larson) }\end{array}$ \\
\hline $1: 15$ & $60 \mathrm{~min}$ & discuss video / $M G$ culture as related to WADA \\
\hline $2: 15$ & $30 \mathrm{~min}$ & preparation for Beta play (overhead walk through) \\
\hline $2: 45$ & $15 \mathrm{~min}$ & break \\
\hline 3:00 & $90 \mathrm{~min}$ & $\begin{array}{l}\text { Bold items eliminated } \\
\text { in Simplified Simulation }\end{array}$ \\
\hline $\begin{array}{l}4: 30 \\
5 \mathrm{pm}\end{array}$ & $30 \mathrm{~min}$ & $\begin{array}{l}\text { Italic items eliminated } \\
\text { in Video Control }\end{array}$ \\
\hline
\end{tabular}

Figure 27. Schedule for the cultural-simulation classes.

Team Assignment. Participants were assigned to teams randomly. Teams had four members, one playing the manager of each department in the simulation, Engineering/Marketing, Sales/Service, Operations/Finance and Support Groups. Some efforts during class enrollment had been made to create class sizes with multiples of four. On days that the number of participants was not, evenly divisible by four the following adjustments occurred: missing one person to fill the final team - either the facilitator or 
the technical support person played to complete the team; missing two persons to fill a team - both the facilitator and the technical support person played; missing three persons to fill a team (i.e., one extra person) - the extra person was paired with another participant to make a five person team, and two participants shared one Macintosh.

Create a Team Name. Each team had their own table and were asked to choose a team name. This was intended to get them to accept the goal of winning the simulation and to allow for sharing of discoveries and observations during the simulation.

Color-coded Hats. The groups were given hats marked with the cultural role (Greek letters Alpha or Beta) and were asked to wear these.

Instructions to Adopt the Role. Players were given instructions to set aside their normal frame of reference and adopt the cultural role. These instructions were printed in the cultural role booklets and read aloud by the facilitator.

Presentation of Role Summary. The teams were given copies of the cultural role they would play, about four pages of single spaced text. Participants were told that their knowledge of the cultural role would be tested with multiple choice questions during the simulation and that they would not have access to the role descriptions while being asked these questions. They were given 20 minutes to read the cultural role and then discuss it among their team. Each team then gave a 3 minute presentation to the entire class summarizing the role using a flip chart.

Facilitator "Walk Through". The participants then moved to their individual Macintosh computers and the facilitator conducted a 15 minute "walk thorough" using his Macintosh to drive an overhead projector. 
Basically the facilitator played the first round of the simulation and called attention to a list of key features in the Facilitator Guidebook (Appendix F) while answering any questions. Participants were asked to follow along entering their own decisions, and thus played the first round of the simulation during this time. This presentation was intended to make certain all participants quickly became familiar with the software interface. This also provided an overview of the rules as a reminder for participants - or to inform any who had not read their rule booklet prior to class. At the end of this "walk thorough" participants moved on to the next round of the simulation and played without input from the facilitator.

Public Score Keeping. At the end of each round the facilitator gathered the cumulative score for each team and wrote these on a flip chart which tracked the scores of all teams. This score was the total points each team had earned by completing simulation tasks. It represented "success" in the simulated business environment. This comparison was placed where all participants could see it and the facilitator called attention to it as he entered the scores. This discussion was intended to convey a sense of competition and get them to focus on 'Winning' as defined by the culture role (the slightly different definitions of winning for the two roles are discussed below). All teams displayed a considerable amount of competitive spirit, a reflection of their world view rather than the effectiveness of this class feature.

"Blender". At the end of rounds two and five the facilitator announced a "blender" and participants left their computers, returned to the team tables, and were given five minutes to discuss with their team members their progress, strategies, observations, etc. ("Blender" is a pun for the "mixers" common at Mentor Graphics. See Appendix C for a discussion of the MGC 
culture.) This was intended to allow participants to review the cultural roles, which they were required to leave at the team tables, and for a cross fertilization of ideas and sharing of participants' discoveries.

Debriefing. There then was a 30 minute period for discussion. The facilitator had a list of questions to ask to ensure that certain topics were addressed (These questions are listed in the Facilitator Guidebook, Appendix F). The questions focused on causal relationships in the simulation and comparison of the cultural roles to subjects' experience at Mentor Graphics.

Cognitive Map Presentation. The presentation of a cognitive map summarizing the simulation lessons has been suggested by several authors (Bowen 1987, Thatcher 1990). A video tape of leaders in the organization taking about the topics and relationships in the simulation was considered a good vehicle to present such a map and simultaneously establish its credibility. This was attempted, however, the video tape that was created did not present a cognitive map that the researcher judged to be detailed enough or explicit enough to convey the model in the simulation. The project sponsor was not willing to omit the video. Therefore four segments of the video were shown. In addition the facilitator presented a series of overhead transparencies which described the simulation model (Appendix G contains an exhibit that summarizes this model). The video segments totaled nearly 30 minutes and were shown while participants ate lunch at the team tables. The overhead presentation was about 20 minutes in length. The video tape, the overhead presentation, and the cultural roles use different media to present a cognitive map of the simulation model to participants. The video tape and overhead presentation immediately preceded the play of the Beta cultural role. 


\section{Class Features of the Beta Game}

The features of the Beta game were very similar to that of the Alpha role as described above: reading the role, discussion in teams, overhead walk through, blenders, and post-simulation discussion. The same teams and team names were used in the Beta game. The differences were that aspects of the simulation unique to the Beta game were described in the "walk through" and whereas in the Alpha game the teams' task point scores were recorded, in the Beta game both task point scores and quality scores were recorded. The quality score is a concept unique to the Beta culture. For Betans, maintaining a minimum quality score (avoiding bureaucracy) is a necessary pre-condition for 'winning'. The questions used to guide the postgame discussions were different because the two roles have different beliefs about 'winning' and about the causal mechanisms in the simulation model.

\section{Class Features for the Other Treatment Groups}

For the Simplified Simulation group, the primary difference was that the Alpha game was omitted and the Beta role was adopted for the first and only game (see Figure 30). The team hats and instructions to identify with the cultural role were also omitted for the Simplified Simulation group. The Video Control group was identical to the Enhanced Simulation group but the video tape was not played during lunch.

\section{VIDEO TAPE DESIGN AND RESULTS}

The creation of a video tape was an option that was discussed with the project sponsor at the start of the project, but the questions of cost and content were not well defined. During the interviews many individuals expressed substantial respect for and confidence in "Tom and Gerry", the 
CEO and president. Statements such as "You couldn't find a better qualified person to do that jcb than Gerry Langeler", or "Tom is really on top of the issues facing the company" were voiced frequently by people throughout the organization during this time period (1987-88).

\section{Plan}

Simulation researchers have commented on the need to present participants with a cognitive map of the model they experience during the simulation. A video tape of the leaders talking about the ideals of the organization's culture was planned with the intent that it could be an effective media for conveying the simulation model. The CEO, President and Executive Vice President, agreed to be actors in the video tape when they approved the frames of reference to be the content for the simulation. Eleven other employees were asked by the researcher to be actors because comments they had made during the interviews supported the frames of reference. These employees were informed only of the cover story.

The video tape was created prior to the start of simulation design and the subsequent de-emphasis of the 'Coaching' and 'Partnership' frames of reference and topics related to all three frames of reference. The format of the video was to have a series of one-on-one conversations in which one of the three leaders discussed a topic with an employee. Figure 28 displays the video storyboard.

\section{Execution}

The project sponsor wanted to minimize the cost of the video tape so the researcher wrote the scripts, operated a camera and did all of the editing. It was expected that the taping could be done in two or three sessions of two to 
three hours length and that these sessions could be completed within 30 days. The initial taping sessions were scheduled for August 1988 but a series of cancelations, postponements and re-schedulings by the leaders resulted in the final taping session occurring in January of 1989. Editing was completed in February.

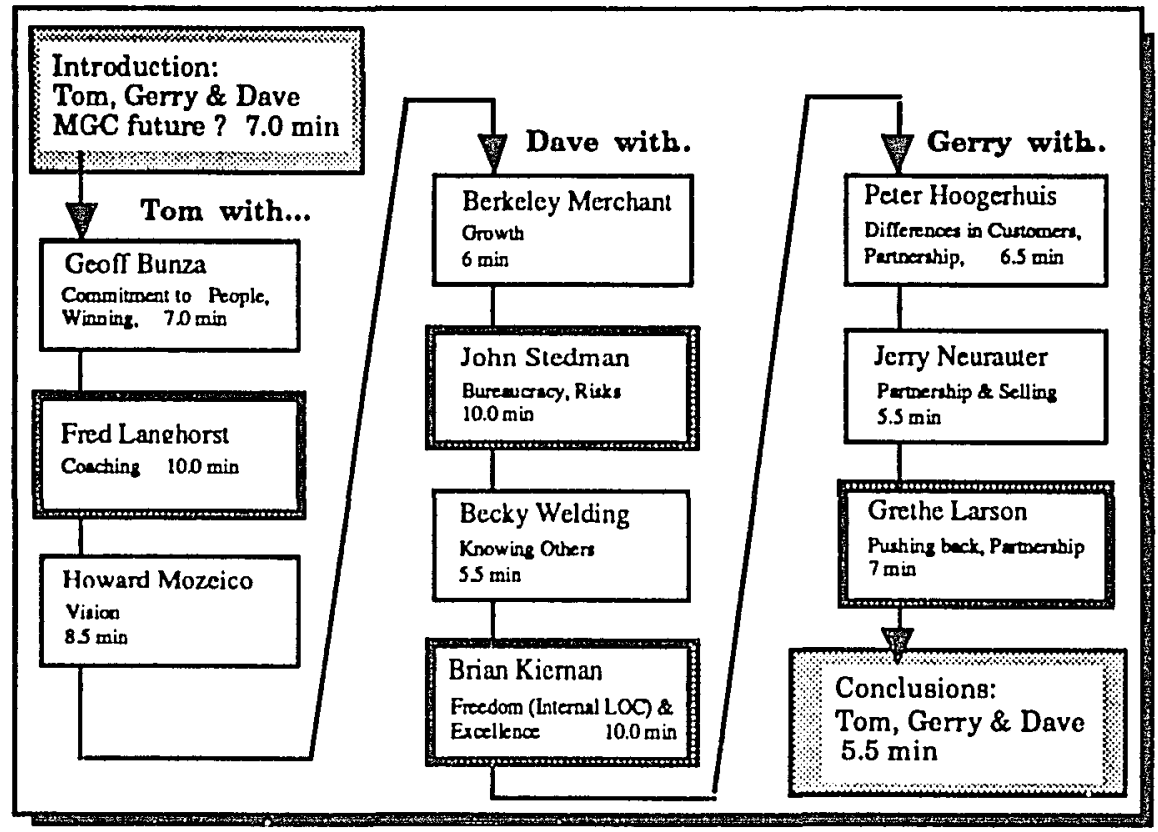

Figure 28. Final storyboard for MGC cultural video.

The Human Resources department created a video shortly after the cultural video for use in new employee orientation. They also waited through several months of postponements in an attempt to get all three leaders, individually, on tape. They settled for only two of three and this seems to confirm both the very busy work schedules at Mentor Graphics and the low priority the leaders placed on making videos to be used in MGC classes.

In addition to the long delay in scheduling the taping sessions the content of the video was disappointing. A total of nearly 10 hours of raw 
footage was recorded and the final video was 90 minutes long. Four segments were shown during the simulation class: Langhorst, Stedman, Kiernan and Larson. These were selected because of the topics discussed, the quality of the acting and the close relationship between the video and the simulation model.

Despite the disappointment in delays and final video content, making the video provided additional opportunity to observe the MGC culture. Only one of the 11 employee actors was scheduled to talk about "pushing back", yet in four of the 11 raw footage segments the actors made considerable reference to the importance of this practice. This triggered a rethinking of this construct that led to its inclusion in the simulation. Also of interest, employees who had made strong statements about the need for change during the interviews - a major criteria for their selection as actors - made statements on those same topics with far less commitment and far less vigor when facing senior management in front of the cameras. This raised questions about how the "open door" policy was practiced at Mentor Graphics.

\section{DESIGN OF THE SIMULATION}

An overview of the simulation is provided by the rule booklet (Appendix E). Several design issues faced during the creation of the simulation, but that were not varied across treatment groups to test their effectiveness, are discussed in this section.

\section{Computer Based}

The decision to make WINNING AT DESIGN AUTOMATION (WADA) a computer based simulation was driven by a strong preference of Dr. Steven Brenner. The client also preferred this option and it was compatible with the 
skills base of the intended participants. In opposition to the computer based approach was a concern of the researcher that the man-computer interaction would dominate the game at the expense of the qualitatively different interactions between persons. ${ }^{18}$ Additional concerns about a computer simulation included the problems of computer availability, the possibility of power outages and the need for technical support. The researcher's intended emphasis on interpersonal interaction, such as is found in the BAFA BAFA simulation, was gradually eroded throughout simulation development.

Once the decision was made to build a computer simulation the selection of Macintosh ${ }^{\mathrm{TM}}$ and HyperCard ${ }^{\mathrm{TM}}$ were the obvious options because of the researcher's familiarity with the former and the promise of the latter. The simulation was written in HyperCard ${ }^{\mathrm{TM}}$ version 1.2.2 on a MacPlus. The slow performance of the simulation was a problem during the development of the software and perhaps $20 \%$ of the programming effort went into rewriting functioning code to improve performance. During simulation development the Macintosh SE 30 became available and the client agreed to rent these machines for the classes. The performance improvement offered by this hardware was somewhat greater than what had been earlier obtained with a great deal of programming effort. Together satisfactory performance, defined as a maximum delay between participant input and final machine response of less than one second, was obtained.

HyperCard is an exceptional simulation development environment. The evolution of the simulation was rapid once programming began and this was

18 The concern about qualitatively different interactions between persons and computers remains a research question in simulation design, one that is not addressed by this study. 
due in a large measure to the object oriented design nature of HyperCard. It permitted playing the simulation and getting feedback from volunteers even though the software was incomplete. The programming to support networking the computers was far more difficult than the initial assessment indicated and one problem was never resolved resulting in using an on-site technical support person for each of the simulation classes. Version 2.1 of HyperCard corrects this problem.

The computer-based simulation format structured class delivery. Once participants were given their instructions, the facilitator had relatively little discretion until the debriefing discussion. A significantly greater preparation effort would have been required from the facilitators had a non-computer format been adopted. In addition, it allowed participants to play as fast as they could assimilate information and make decisions. This is in contrast to some other simulations in which participants must wait while facilitators manually update simulation status ${ }^{19}$. Beyond the reduced facilitator preparation requirement and quick response, adopting a computer based format is believed to have had little impact on simulation effectiveness.

\section{The Macro Level Structure}

The primary design feature of WADA is having participants experience the two contrasting cultural roles, Alpha and Beta. This feature has its theoretical basis in Bennett's model of empathy (see Figure 10, p 37) and empirical precedence can be found in both the BAFA BAFA simulation (1977) and the suggestions by Fletcher (1971). The contrasting cultural roles are

19 See for example ZAN-TEC by Richard Powers or POWERPLAY by Jeff Wright. 
experienced sequentially in two plays of the simulation. Participants who adopt these roles in the successive plays of the simulation learn how the roles interact with the simulation model and test these roles as alternate strategies for winning. The simulation model produces different outcomes from patterns of participant decisions that are consistent with the different cultural roles.

The Alpha culture (first play) is similar to the existing Mentor Graphics culture in that there is a passive acceptance of growing bureaucracy. Playing this cultural role results in an outcome also consistent with the beliefs of the existing MGC culture - the simulated company becomes "bureaucratic", an unpleasant condition. In the Beta culture, participants actively work to maintain the small company atmosphere. They see how certain actions they take cause their department to avoid becoming "bureaucratic". A higher total score results when following the Beta role because the resource drag of bureaucracy is avoided. The Beta role was designed to be both a dominant strategy (more points could be earned than with the Alpha strategy) and a more enjoyable role (the Alpha strategy leads to bureaucratic frustration). However Beta is not an obvious strategy for the novice player and the role, simulation identification features and facilitator guide participants to it.

\section{Role Identification Features of the WADA Simulation}

At a level of simulation structure more detailed than the two contrasting role/strategies, many of the design features are intended to support participants' learning, practicing and identifying with the cultural roles. The class begins with reading the cultural role descriptions, discussing these in teams and presenting a summary to other teams. This is the start of the 
multiple presentations of the cognitive map of the simulation model including the cultural roles, the overhead slide presentation, and the video tape.

To encourage reference to the cultural roles during play, the simulation asks participants multiple choice questions about the cultural roles ${ }^{20}$. Correct responses lower the cost to participants of getting points. WADA also monitors player inputs on key decisions (for example the amount of resource devoted to "building the vision"). If a player's inputs are outside a predetermined 'normal' range for the cultural role, WADA provides immediate feedback reminding the player of the role.

Color-coded hats labeled with the name of the cultural role are presented to players along with instructions to adopt that role. These props are to support the participants in setting aside their normal frames of reference and adopting the cultural roles. The "identify" instructions were created following Williams (1987).

The cultural roles are carefully constructed to contrast along the attitudes and beliefs where change is intended. For example, both cultural roles state a strong belief that the vision of the company is important and this is consistent with the existing MGC culture. However the Alpha culture relies on senior management to provide most of that vision, while the Beta role has individuals accept responsibility for, and make greater contributions to, the vision.

The Beta cultural role (presenting the frame of reference the simulation attempted to convey) is constructed to be consistent with attitudes and beliefs of the existing MGC culture that were not targeted for change. For example,

20 This multiple choice feature was suggested by Plumber (1982). 
"bureaucracy" has a very negative connotation in the MGC culture and this connotation is not a target for change. This dislike of bureaucracy is central to the Beta cultural role. Parallels between existing attitudes at Mentor Graphics and the frame of reference suggested by the simulation, such as the dislike of bureaucracy and the importance of vision, make it easier for player's to identify with both roles and to incorporate the Beta role into their organizational cognition. A general design strategy was to balance the contrast between roles with consistency with the existing culture.

The content expressed in the Alpha and Beta culture descriptions are internally consistent, self-reinforcing, systems of beliefs. (The Beta role is a prose description of the "Quality Growth" frame of reference described earlier, p 75-77, and is presented in Figure 18). It is believed that the presentation of a more fragmented belief structure would reduce the likelihood that players will adopt it. In addition, Alpha and Beta are designed to be consistent with many pre-existing participant attitudes in order that they both be perceived as legitimate cognitive systems. The close observation of the corporate culture in Phase 1 of the study provided the raw material to support the incorporation of these attitudes in the roles and the simulation model.

Graphs of the simulation model's state variables are available to participants during the simulation. These include the level of vision, partnerships, risk taking, rewards, coaching and pushing back. The Alpha and Beta cultural roles have different perspectives on these variables and this is represented by two different graph formats. During the Alpha play only Alpha graphs are available. During the Beta play both the Alpha formats and the Beta formats are available to participants. Having already 
learned the Alpha role in the first game, they can refer to that view while learning the Beta role in game two. The presentation of two graph formats enhanced participants' awareness of the contrasts between the roles.

Each participant's performance during the Alpha play is recorded and plotted on the Beta graphs so that participants see later how the pattern of decisions they made playing the Alpha role would be perceived in the Beta culture. This is an elaboration of Fletcher's (1971) recommendation of providing a recording mechanism and multiple plays, here intended to strengthen the the player's awareness of the contrast between roles. Plotting the Alpha behavior on the Beta graphs was also expected to create a dissonance condition in those treatment groups who played both cultural roles. Figure 29 shows an example of a participant's Alpha and Beta history plotted on a Beta graph. Note the substantially different behavior for the two roles. Participants see that the pattern of decisions which they made in the Alpha play resulted in a adverse condition - their department became "bureaucratic". It was expected that this dissonance would result in participants realizing the need to change their behavior and then changing their attitudes relating to these behaviors.

The goals of the Alpha and Beta cultures are slightly different and this is an additional example of defining alternate realities with these frames of reference. In the Alpha play the goal is only to obtain the most points. In the Beta play, participants strive to obtain the most points with the additional constraint that if they lose their small company atmosphere - as measured in the Betan's Quality graph of Figure 29 - there is a severe penalty assessed to their point totals. 


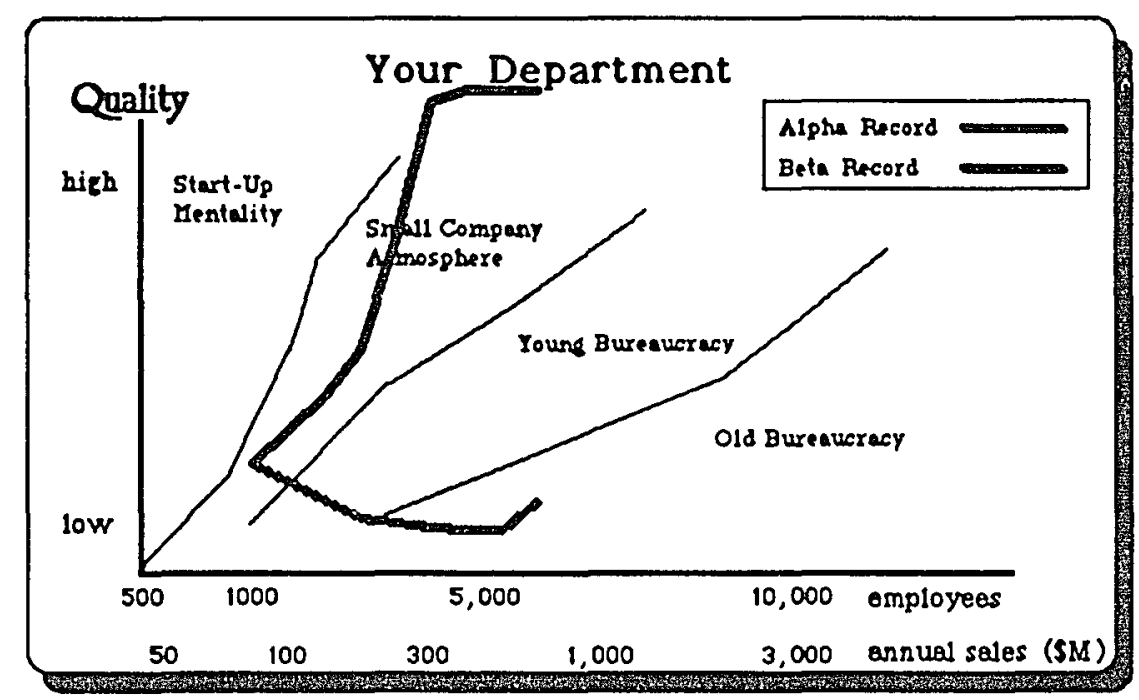

Figure 29. Example quality graph at end of Beta game.

The design of the model that drives the behavior of the WADA simulation, as well as much of the content of the simulation, is based upon information gathered in the ethnographic interviews in Phase 1 of the study. Terms incorporated in the simulation, e.g., vision, bureaucracy, small company atmosphere, pushing back, were drawn from the interviews. The primary resource in the simulation is 'time' - and not money as in most business simulations - because of the importance of time in the organizational culture. Another example is the size of the simulated company. Initially there are 900 employees - compared to about 2,500 at MGC - and at the end of the simulation there are nearly 7,000. Participants can maintain the small company atmosphere during this simulated growth and it was intended that they would incorporate this experience into the mental model they use is assessing whether MGC can maintain its small company atmosphere. The many instances of incorporating the ethnographic 
knowledge into the simulation might be considered a "simulation design stylen.

\section{Simulation Design Style}

This simulation design style is consistent with the goal suggested by Constructivism, which is the creation of a rich set of stimuli to support parícipants as they learn an alternate reality. Elaborate props and stages along the lines of a theatrical metaphor (Goffman 1959) are consistent with this design perspective. ${ }^{21}$ It stands in contrast to what was offered as the preferred design style at the 1988 NASAGA conference (Goodman 1988). There it was repeatedly suggested that "Simulations should be designed so that you can get all the necessary parts in a hardware store for less than $\$ 50$. . . This could be termed the "minimal resource" simulation design style. The goals of this approach include simplicity in physical representations of game content, low cost and ease of game element replacement. This last point was discussed at the conference with references to gaming sessions where some critical part was lost and the session was jeopardized by difficulty of replacement. This approach is driven by pragmatic considerations related to running simulations.

The alternate to this "minimal resource" style suggested in this study is based on a theoretical perspective. The video tape, networked Macintosh computers, and elaborate HyperCard program, all incorporating materials from the ethnographic interviews, are design choices in direct contrast to the

21 It is not the purpose of this study to answer the question of which design style is 'better'. Instead the formulation of this question, the suggestion of an alternative to the "minimal resource simulation" design is a question raised in this study. 
"minimal resource" style. The WADA design style might be labeled

"elaborate simulation" and the goal is presentation of rich, intense realities.

Suggesting a Preferred Cultural Role - Beta

The WADA simulation is designed not only to convey the contrasting Alpha and Beta frames of reference, but also to convey that one frame of reference - Beta - is preferred to the other. Without design features to suggest this, it would be logical to expect that the simulation might have an effect that was the inverse of its design goal and move participants toward the Alpha frame of reference. The goal of the WADA simulation was to move participants toward the Beta (Quality Growth) frame of reference.

There are four features used to convey the preferability of the Beta cultural role. First, the leaders of Mentor Graphics are shown conveying portions of this frame of reference. The two treatment groups that saw the video therefore saw role models suggesting these are the ideals of the organization as suggested by the executive group and the other actors in the video. Second, the simulation model is structured so that the Beta cultural role will out perform Alpha. On average participants will score $20 \%$ more points when adopting the Beta role/strategy than they did in Alpha. Third, the outcome of playing the Alpha cultural role is unpleasant, the simulated company becomes bureaucratic and this is frustrating to participants. Adopting the Beta role avoids this and thus is a more enjoyable role. Finally, the design of the Beta frame of reference is consistent with many facets of the existing Mentor Graphics culture. The trend toward bureaucracy, which was always evaluated negatively in the interviews, is overcome when adopting the Beta culture. Participants who choose to adopt the Beta role are empowered 
to maintain their small company atmosphere and an internal locus of control regarding organizational growth.

\section{Dynamics of Winning At Design Automation}

Participants in both the Alpha and Beta roles attempt to maximize their points, thereby beating the competing teams and winning. Each participant must discover what strategy will result in the best score and the cultural roles guide them into experiencing two different strategies.

The simulation evolves into one of two different behavioral states depending on the pattern of the participant's choices. The two cultural roles channel participants into experiencing these two states. None of this is obvious to participants. While the cultural roles provide some guidance for making decisions about resource expenditure, participants perceive that they are selecting the best strategy. Only as the model is discovered and the strategy contained in the Beta role revealed does Beta appear as a viable, and eventually even a superior, option.

Playing the Alpha role/strategy results in the company gradually becoming bureaucratic. This is represented in the simulation by an increasing amount of "rules and procedures" required to accomplish tasks, a series of messages conveying the feeling of bureaucracy, and forcing participants to more and more frequently search the "bureaucratic maze" for information. The maze in particular is designed to give participants the experience of an external locus of control, i.e., "bureaucracy" as defined in the MGC culture. Participants also observe some of their resources being drained off by bureaucracy.

The Beta cultural role includes a greater emphasis on maintaining the small company atmosphere. Greater amounts of time are invested in vision, 
coaching, risk taking, partnerships, pushing back and rewards. The result is that bureaucracy and the maze are avoided. A higher point total results than when playing the Alpha role because the organization is functioning at a higher level of effectiveness. In the debriefing the facilitator asks questions to suggest that the important comparison for participants to make is not their score against that of other teams, the obvious one during play, but the difference between their own Alpha and Beta scores.

\section{EXPERIMENTAL PROCEDURES - PHASE 4}

\section{Definition of Treatment Groups}

The WADA simulation contains two cultural roles, Alpha and Beta. When the simulation is played in its full form (Enhanced Simulation and Video Control treatment groups), players adopt the Alpha cultural role in their first play of the simulation and then adopt the Beta cultural role in a second play of the simulation. Giving the players the contrasting views of these two roles was a design feature suggested by the communication theory used in this study. Briefly, the content of the Alpha cultural role is very similar to the existing Mentor Graphics culture while the content of the Beta cultural role presents some of the ideals of the Mentor Culture. The intent of the simulation is to cause attitude change in the direction of becoming more like the Beta cultural role.

Originally there was to be two treatment groups, an Enhanced Simulation and a Simplified Simulation, the former expected to cause the greatest change. The video tape was a possible feature to be included in the Enhanced Simulation with the expectation that it would be paired with an audio or printed equivalent in the Simplified Simulation. The client agreed 
to fund the video tape but considered the idea of using the "less impact" audio or print options to appear contrived. It was decided that the video would be used for all treatment groups and that other simulation attributes would be varied to create enhanced and simplified simulation classes. It then became obvious that if change did occur, it could be questioned whether the change was due to the simulation or, perhaps, only the video tape. The client was willing to solivit additional employees for a third treatment group, one that would receive identical conditions to the Enhanced Simulation group with the exception that the video tape would be omitted. Figure 30 lists the attributes of the simulation class that distinguish the treatment groups.

\begin{tabular}{|c|c|c|c|c|}
\hline Altributes & $\begin{array}{l}\text { Enhanced } \\
\text { Simulation }\end{array}$ & $\begin{array}{l}\text { Video } \\
\text { Control }\end{array}$ & $\begin{array}{l}\text { Simplified } \\
\text { Simulation }\end{array}$ & Control \\
\hline Video Tape & Yes & No & Yes & No \\
\hline $\begin{array}{l}\text { Contrasting Alpha } \\
\text { Culture Role }\end{array}$ & Yes & Yes & No & No \\
\hline Culture Hats & Yes & Yes & No & No \\
\hline $\begin{array}{l}\text { "Empathize" } \\
\text { instructions }\end{array}$ & Yes & Yes & No & No \\
\hline $\begin{array}{l}\text { Beta Culture Role } \\
\& \text { all other } \\
\text { Simulation } \\
\text { Attributes }\end{array}$ & Yes & Yes & Yes & No \\
\hline
\end{tabular}

Figure 30. Simulation class attributes and treatment groups.

The Simplified Simulation group did see the video but differed from the other treatment groups along three attributes. The primary difference was that this group did not play the Alpha cultural role in an initial play of the simulation and therefore was not presented with the contrast of two cultural roles. Instead they experienced only the Beta culture comprising the 
attitudes of the organizations' ideals. This meant that they did not experience any dissonance from seeing their behavior in the Alpha game as "Betans". The absence of the contrasting roles was expected to diminish role identification in the Simplified Simulation group. In addition the Simplified Simulation group did not receive Culture Hats, a prop intended to create greater role identification. They also did not receive the instructions to set aside their normal world view and empathize with the cultural role.

\section{Control Group}

The Control group was included to see if some event at Mentor Graphics caused change in participant attitudes, or if simply filling out the questionnaire caused them to change their responses. Filling out the preand post-simulation questionnaires was the only involvement for this group. Post-simulation questionnaires were sent to this group at the same time as the last treatment group and, along with that group, they had the longest period between questionnaires (five months).

\section{Assignment of Subjects to Treatment Groups}

The subjects in the experiment were employees of Mentor Graphics who worked in the Beaverton headquarters. Pre-simulation questionnaires were sent to employees whose names were randomly drawn from the payroll list. A total of 354 questionnaire packets were sent via company mail in late May of 1990 . Included in the packet was a cover memo from the CEO requesting cooperation with the study and one from the principal investigator providing instructions and assuring confidentiality (Appendix D). Also included in the packet was a stamped envelope addressed to the home residence of the 
principal investigator. By the middle of June, 216 useable questionnaires (61\%) had been returned.

An average score for each subject was calculated for the 14 items in the Davis empathy scale. Subjects were ranked using this average score and were assigned to one of four groups to create a block design with the empathy scores the blocked variable. This was done to ensure that the distribution of empathy scores was roughly uniform. These four groups were then randomly assigned to be one of the three treatment groups or the control group.

The lists of names in the three treatment groups were presented to the project sponsor in early July. All invitation activities were conducted by the Human Resources department of Mentor Graphics because of the goal of having subjects remain unaware of the principal investigator's association with the simulation. Each person in the treatment groups was sent a one page memo in the company mail from the project sponsor inviting them to a one day workshop on corporate culture. That memo indicated that because Mentor Graphics was approaching its tenth birthday it was appropriate to "revisit our organizational culture".

Each person in the treatment groups was contacted by phone about eight days before the simulation class to determine if they would attend. Subjects were offered a choice of attending on either of the days for their treatment group. Subjects were not aware that the simulation class on some days would be different from others. Those who agreed to attend a simulation class were sent a packet four days before their class containing a cover letter from the project sponsor, the simulation rules booklet (Appendix F) and a map to the Valley Conference Center where all the classes were held. The rental of the 
valley conference center, the 21 Macintosh computers, and lunch arrangements were organized and paid for by Mentor Graphics.

\section{Simulation Class Sequence and Schedule}

A full day was scheduled for each simulation class. It was expected that there would be approximately 20 subjects in each class and scheduling two classes for each treatment group was expected to yield nearly 40 subjects in each treatment condition. Six classes were planned (2 classes x 3 treatments).

The sequence for the treatment group classes was determined by several considerations. First, because the simulation is complex, it would require considerable attention from the facilitator. The differences between the treatment group classes would add an additional level of facilitation complexity. To minimize this, it was decided to have consecutive pairs of days with the same treatment conditions. This conclusion ran counter to a purely scientific approach of mixing treatment conditions to balance any practice effect for the facilitator. However it was judged to be the best approach given the minimal time the facilitator would devote to preparation for the classes.

Some of the treatment groups would be easier to facilitate than others. (Figure 27 displayed the schedule for the fullest day.) Other days had fewer activities (Simplified Simulation had no Alpha game and Video Control skipped the video and its discussion). The very full day schedule suggested scheduling the easier classes first to best allow for learning this simulation.

Another consideration was the possibility of subjects who completed the class influencing others who had not yet attended. (At the end of each class the facilitator made a statement to all participants asking them not to 
disclose the Alpha and Beta roles to others who had not yet played because it would diminish their learning experience. The cultural roles descriptions were also collected in order to reduce the chance that other subjects would learn about them.) This concern had two dimensions. The greatest concern was that people who had played the Beta role would convey this role to others who would then adopt the Beta role, a superior strategy, when they were asked to play Alpha. A second concern was that subjects in the Simplified Simulation treatment condition could be aware they were receiving a different treatment if they had talked with other subjects and guess they were subjects in an experiment. This could influence their responses to the questionnaire.

These considerations were best handled by presenting the Simplified Simulation treatment last. The "Beta conversion" concern was deflected as the subjects in the last classes (who had more time to interact with subjects in previous classes) would not be asked to play the Alpha role. Regarding the concern about modified class content. the facilitator could respond, "After running the simulation several times it seems that most of the learning occurs in the Beta role. Thus I can save you all several hours by having you play only the most effective part of the simulation." This would be consistent with the time pressure at Mentor Graphics, and the cover story that the simulation was supplied by an "external vendor" with the Human Resources department expected to modify it based on experience. Together these considerations lead to a class sequence in shown in Figure 31.

Only about half the number of subjects attended the August classes as had been expected. In addition a technical problem resulted in the early termination of the first class and no data was gathered from these subjects. 
A second session of classes was scheduled for October to offer the simulation to more subjects.

The October date was selected as this was the next week where the project sponsor's schedule allowed him to conduct another week of classes. It was considered an important factor to have the same facilitator for all sessions and thus the two month delay was accepted to keep this factor constant. However a week before the October classes the project sponsor announced that he would travel to Singapore during October and offered that either another person would substitute as the facilitator or the classes could be re-scheduled for December.

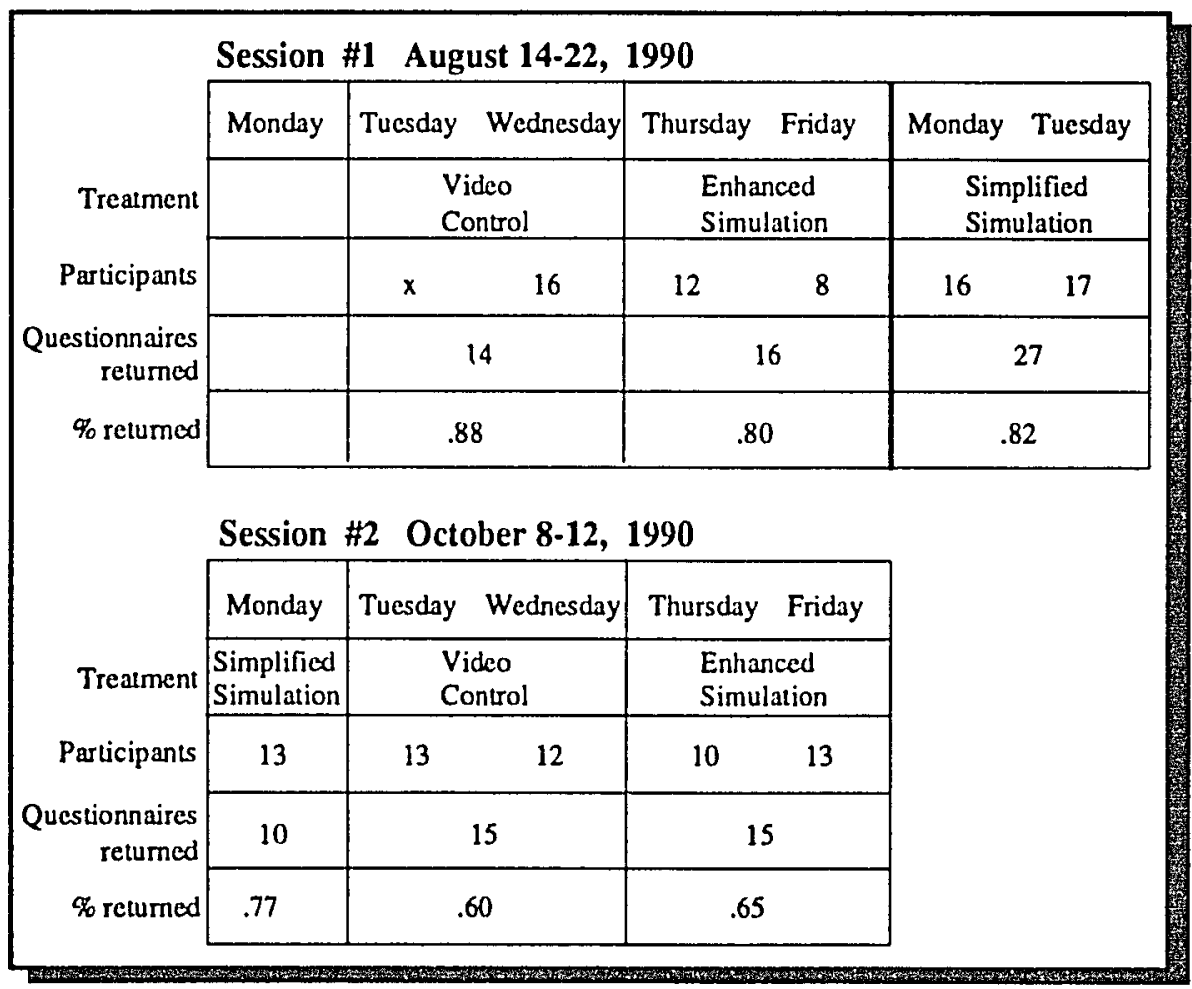

Figure 31. Simulation class schedule for each session. 
This raised the issue of facilitator effectiveness as a confounding variable even though some subjects in each treatment group could be scheduled for both facilitators. In addition the impact of facilitator learning effect - made obvious during the August session - would be magnified. After already endured one two-month delay and considering the possibility of yet another delay in December, the substitute facilitator was accepted.

The distribution of subjects by treatment group in the August session indicated the need to schedule more Enhanced Simulation and Video Control classes with less need for additional Simplified Simulation classes. The performance of the subjects in the August session substantially reduced the fear that players would avoid the Alpha role or "convert" to a Beta role in searching for a better strategy. (No individuals in the August session attempted a Beta strategy while playing the Alpha role.) This led to the October class sequence shown in Figure 31.

\section{Facilitator Guidebook}

A guidebook was prepared for the facilitators (Appendix F). The purpose was to remind the facilitators of the day schedules and in particular the differences between the treatment group classes. The guidebook lists discussion questions for each of the discussion periods in an attempt to standardize the topics addressed across the classes. It also lists important activities (hand out hats, collect cultural roles, ask participants to answer multiple choice questions, etc.)

The complexity of the simulation and the desire to limit playing time to 90 minutes for each game led to the decision that the facilitator lead the class in a "walk through" of the first quarter of play on an overhead projector. The guidebook contains a list of features to be emphasized during this "walk 
through" and it helped participants move quickly down the learning curve. It took about 20 minutes to conduct the "walk through" and play the first quarter. Players took about 10 minutes for the second quarter and five to eight minutes to play each subsequent quarter indicating a sharp learning curve.

\section{Simulation Delivery}

Neither facilitator had conducted the WINNING AT DESIGN AUTOMATION simulation, or any other computer simulation, prior to the class sessions. Each had played the simulation in an individual setting once and played it once in a four-person practice session that doubled as a test of the network software. Their busy schedules caused the cancellation of practice facilitation sessions. The principal investigator expressed the opinion that a considerably greater effort should go into facilitator preparation for these classes but that effort was not forthcoming.

One pattern that was common for each facilitator was that in the first few classes they were unable to move the participants through the intended eight quarters of play. These classes played the simulation for only six or seven quarters. By the third class, and in all subsequent classes, each facilitator was able to have the class play all eight quarters. This represented facilitator learning about how to deliver this simulation.

Participants did play faster in the Beta role. This likely reflected the added difficulty in managing a department as it became bureaucratic. This did contribute to one problem during the October session. For three days participants played only six or seven quarters (of the intended eight) in the Alpha role but then played the entire eight quarters in the Beta role. The software was designed to compare results for the last quarter played in the 
Alpha and Beta games - it had been assumed that these would always be the same number of quarters. When different numbers of quarters were played, participants can not easily compare their results across games. The purpose of the comparison was to establish in participants' minds the superiority of the Beta strategy over that of Alpha. This was considered a critical point during simulation design - that subjects knew they played against exactly the same simulation events in both games and that the superior results in the Beta game were due to the superiority of the Beta strategy. The facilitator in the second session did not share the belief that this comparison was important. Despite feedback for the principal investigator, he continued to play different numbers of quarters in the Alpha and Beta roles until he was able to move the class through all eight quarters in both roles.

Players alternated between team activities (reading cultural roles, discussions, lunch, viewing video tape) at the team tables and working alone at their computers. The layout dispersed team members (one in each of the four departments) around the room so that players sat next to competitors. Teams displayed a great deal of competitive spirit and players often coached their teammates. The layout of the class room is displayed in Figure 32.

\section{Post-Simulation Questionnaire}

Fifteen days after each class, the post-simulation questionnaires for the participants in that class were placed in the company mail. All of the control group post-simulation questionnaires were sent out with the last class in the October session to ensure that any external factors that might influence any of the treatment group participants would also have the opportunity to influence the control group. As with the pre-simulation questionnaires, cover 
letters from the $\mathrm{CEO}$ and the principal investigator were included (Appendix D). Participants were asked to return the completed questionnaire within 10 days. A stamped envelope addressed to the home of the principal investigator was included to further imply that the responses would be confidential. The average time for return of the questionnaires was about ten days.

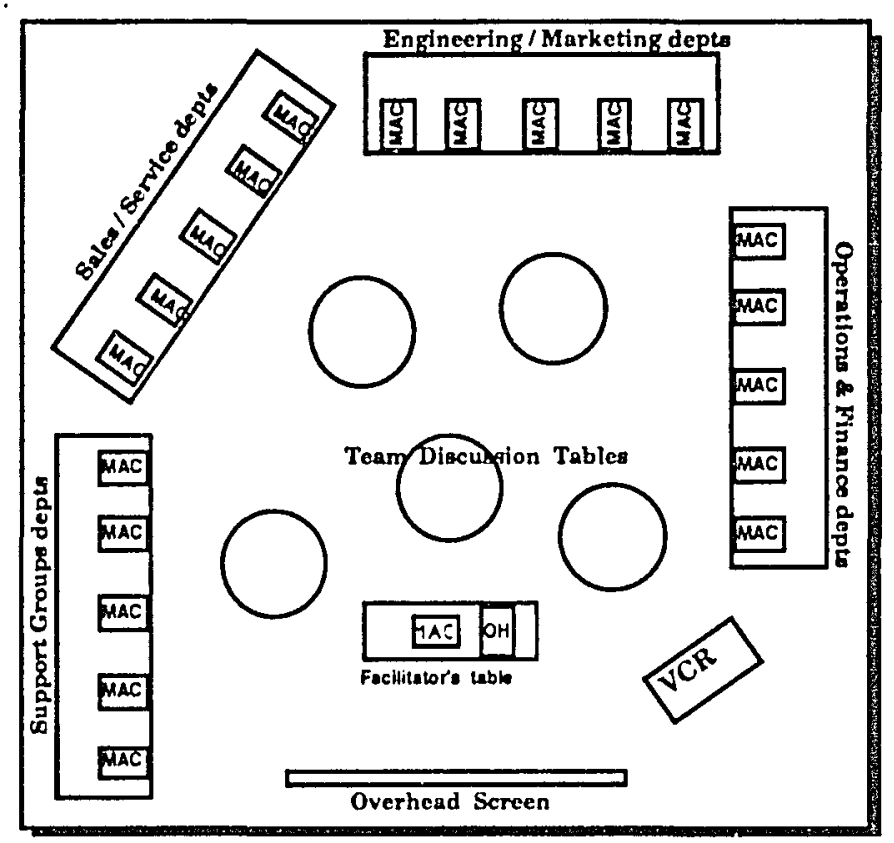

Figure 32, Layout of the simulation classroom.

\section{Post-Simulation Interviews}

Fifteen participants were contacted after all the questionnaires had been returned to elicit comments about the simulation. Of particular interest was whether participants made any connection between the simulation class and the questionnaires. None of those contacted were aware that the simulation class was a part of the student's dissertation study. The usual reaction was complete surprise - even disbelief - followed by congratulatory comments on a job well done. Several of the participants offered that they thought 
everyone in the entire company should play the simulation. A few said it was the best training class they had ever taken at Mentor Graphics. These comments were consistent with the evaluations collected at the end of the class (reported below) and comments during the debriefings.

About a dozen participants returned their post-simulation questionnaires blank along with a note stating that they must have received it by mistake - they had filled out this questionnaire a few months earlier. This further supports the conclusion that participants were unaware of being in a pre-post experimental design. 
CHAPTER V

DATA ANALYSIS

\section{QUESTIONNAIRE STRUCTURE AND RELIABILITY}

The questionnaire was designed to measure change toward the Quality Growth frame of reference. Forty questionnaire items were grouped into eight attitude scales during the creation of that frame of reference as described in the Methodology section (Figures 18 to 26). These eight scales became the primary guide for selection of simulation content. The analysis of those forty items can be approached at several levels. The most global approach is to combine all 40 items into a single measure. A more detailed approach is to examine the eight attitude scales that were defined during the design of the Quality Growth frame of reference. The other extreme is to examine each questionnaire item individually.

Table I displays the correlation matrix for the eight attitude scales and Cronbach's Alpha. The equation to calculate Alpha $=\mathrm{N} \Omega /[1+\Omega) \mathrm{N}-1)]$ where $N$ is the number of items and $\Omega$ is equal to the mean inter item correlation (Carmines and Zeller 1979). Alpha represents a lower bound on the reliability of the questionnaire items and is the most commonly used measure of internal consistency. The average correlation between questionnaire items and the number of items in a scale determine its Cronbach's Alpha value. The rule of thumb is that Alpha should be above 0.6 
for a new instrument and above 0.7 for a previously developed instrument which has been modified to improve its reliability.

The Alpha calculated in Table $I$ is used as an empirical measure of the independence of the eight scales. A low Alpha would suggest that the eight scales be analyzed separately. The value of .75 is high enough to indicate that a global 40-item scale, labeled "Quality Growth", be used as the primary measure of subjects' attitude in the analysis.

TABLE I

DEPENDENT VARIABLE CORRELATION MATRIX

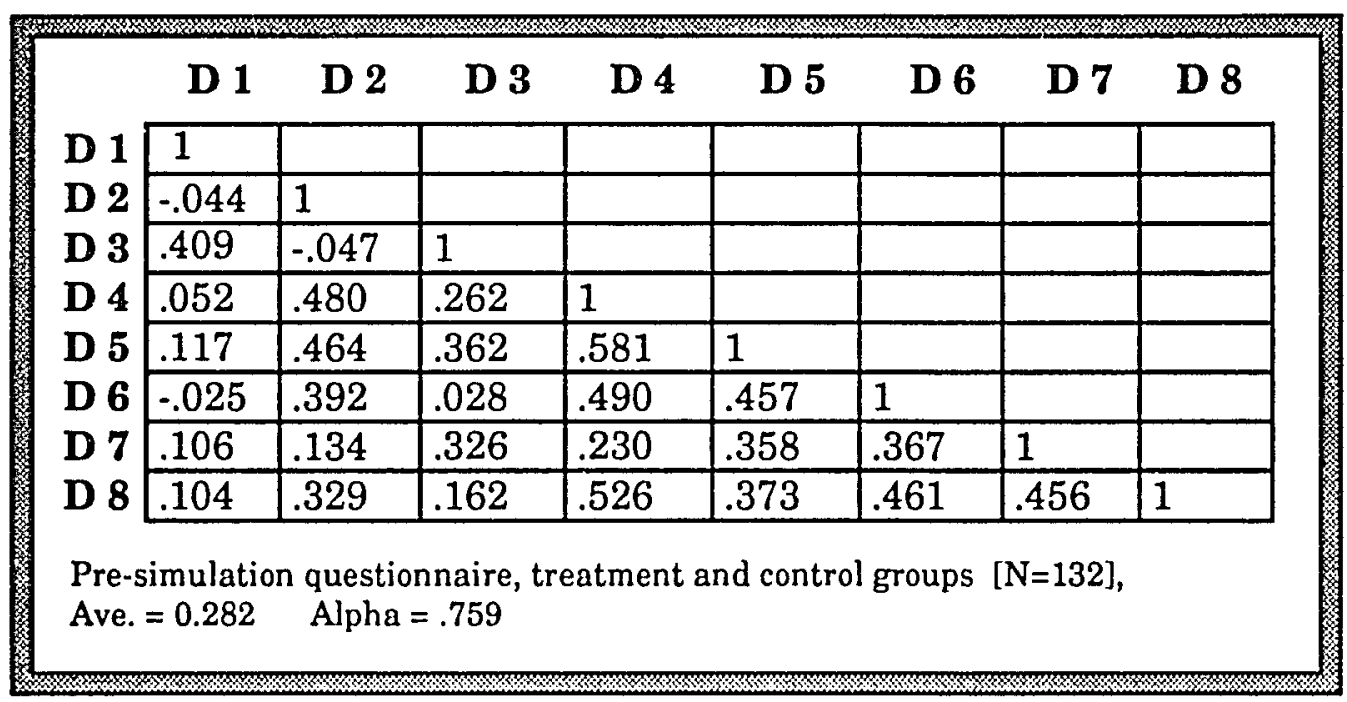

A finer grained analysis may provide additional insight into some questions. Cronbach's Alpha was also calculated for the eight attitude subscales and the results are presented in Table II. All items have Alpha scores that indicate acceptable reliability for an instruments initial use. 
Expectations of Change for the Attitude Subscales

The research questions and hypotheses in Chapter 3 were written prior to collecting any data about the client organization or the design of the simulation. It was anticipated that there would be multiple measures of subjects' attitudes but it was impossible to define whether there would be any differential change expectations associated with these.

TABLE II

CRONBACH'S ALPHA FOR DEPENDENT VARIABLES

\begin{tabular}{|lcc|}
\hline Dependent Variable & Items & Alpha* \\
Quality Growth & 40 & .759 \\
1 Possible to Avoid Bureaucracy & 5 & .655 \\
2 Bureaucracy Definition & 5 & .738 \\
3 Opportunities to Act & 4 & .710 \\
4 Responsibility to Act & 4 & .681 \\
5 Example Actions & 7 & .804 \\
6 Need for Change & 6 & .739 \\
7 Partnerships & 4 & .619 \\
8 Coaching & 5 & .735 \\
* Cronbach's Alpha calculated using scores for all subjects ( $\mathrm{N}=135)$
\end{tabular}

After the simulation was complete a more detailed assessment was made. "Partnerships" and "Coaching" received reduced emphasis during simulation construction as discussed in the Methodology section. The expectations for change in these subscales was therefore low. The capstone variable, asking whether it was "Possible to avoid bureaucracy", was also considered less likely to change. This was due to the broad claim of these items, which made them more open to refutation from non-simulation experiences. 


\section{RESULTS}

\section{Research Question 1}

Are demegraphic variables related to simulation-induced attitude change?

Summary of Findings for Research Question 1. Differences in 'Simulation Delivery' between the August and October sessions had little measurable impact on attitude change. The Simplified Simulation group had the greatest irregularities, attributed to facilitator learning, but there was no significant differences in the Quality Growth dependent variable between sessions for the treatment groups.

Females showed slightly more change than males for most dependent variables though only two of the subscales had significant differences. The one variable for which females had significantly less change was "Opportunities to Act". Gender was not uniformly distributed across treatment groups. The Simplified Simulation group was 50\% female, a considerably higher proportion than the other groups.

Subject age was a not a significant factor predicting attitude change for the Quality Growth variable, though two of the subscale variables measured significant differences. Older employees demonstrated less change. For the "Example Actions" subscale, age accounted for nearly $13 \%$ of the variance. Age was uniformly distributed across treatment groups.

Length of employment in the client organization was a significant factor predicting attitude change for the Quality Growth variable. The amount of variance it accounted for was small, about six percent. Employees who had been with the organization longer tended to have greater attitude change. 
Overall, the demographic factors are unlikely to introduce large distortions in subsequent data analysis. The effectiveness of the WADA simulation appears to be relatively stable in this population even with substantial differences in 'Simulation Delivery' variables.

General Discussion of Findings for Research Question 1. Subject sex, age and length of employment in the client organization were analyzed to search for any unexpected influence. In addition, a 'Simulation Delivery' variable indicating whether subjects were in the August or October session was analyzed to discover if some difference in the delivery of the simulation influenced subjects' attitudes. There are no hypotheses associated with this research question.

Simulation Delivery. The need to compare results from the August and October sessions is suggested by a set of factors that were labeled 'simulation delivery'. Included are possible training effects for the facilitators, nonfacilitator improvements in simulation delivery and differences between the facilitators. A facilitator training effect might be an important factor influencing other analysis reported below because a large portion of the subjects in the Simplified Simulation group (27/37 $=73 \%$, Figure 31) were in the August session. These were classes number five and six for the first facilitator and so the facilitator had had one week of practice delivering the class. In contrast, most of the Video Control group took the first or second class from one of the facilitators, a time when they had no experience delivering the simulation. The facilitators acknowledged that their delivery improved throughout their week of leading these classes.

There were also problems with the network software during the first two classes in the August session. These caused delay as some data had to be 
manually transferred by the technical support person. This lowered the enthusiasm of participants. Improved technical support eliminated this problem by the third class. Finally, the facilitators differed in style, credibility and organizational status.

Table III presents an ANOVA comparison of mean change scores by session for the Simplified Simulation group. There was no significant difference for the Quality Growth variable, the most global measure of subjects' attitudes. The mean change scores are uneven for the two Simplified Simulation sessions, on the eight subscale variables. However the differences are significant beyond the .05 level for only one of these.

TABLE III

COMPARISON OF SESSIONS 1 AND 2: SIMPLIFIED SIMULATION

\begin{tabular}{|c|c|c|c|c|c|}
\hline Dependent Variables & $\begin{array}{l}\text { Aug. } \\
\mathrm{N}=27\end{array}$ & $\begin{array}{c}\text { Oct. } \\
\mathrm{N}=10\end{array}$ & $\begin{array}{l}\text { Aug. } \\
\text { - Oct }\end{array}$ & $\mathrm{F}$ & $\mathrm{P}$ \\
\hline Quality Growth & .516 & .455 & .061 & .04 & .847 \\
\hline 1 Possible to Avoid Bureau. & .500 & .244 & .256 & .55 & .463 \\
\hline 2 Bureaucracy Definition & .208 & .956 & -.748 & 4.59 & $.039^{\bullet}$ \\
\hline 3 Opportunities to Act & .417 & -.250 & .458 & 2.70 & .109 \\
\hline 4 Responsibility to Act & .590 & .475 & .115 & .24 & .625 \\
\hline 5 Example Actions & .803 & .730 & .073 & .06 & .800 \\
\hline 6 Need for Change & .616 & .834 & -.218 & 1.00 & .325 \\
\hline 7 Partnerships & .410 & .139 & .271 & 1.26 & .270 \\
\hline 8 Coaching & .185 & .280 & -.095 & .39 & .538 \\
\hline$\cdot p<.05$ & & & & & \\
\hline
\end{tabular}

Table IV shows that the change scores for the Enhanced Simulation group are more uniform across sessions, though this leaves open the question of whether the delivery was uniformly good or uniformly poor. Again there is 
no significant difference between sessions for the Quality Growth variable. Differences measured by the subscales are also insignificant.

\section{TABLE IV}

COMPARISON OF SESSIONS 1 AND 2: ENHANCED SIMULATION

\begin{tabular}{|c|c|c|c|c|c|}
\hline Dependent Variables & $\begin{array}{c}\text { Aug. } \\
N=16\end{array}$ & $\begin{array}{c}\text { Oct. } \\
N=14\end{array}$ & $\begin{array}{l}\text { Aug. } \\
\text { - Oct }\end{array}$ & $\mathbf{F}$ & $\mathbf{P}$ \\
\hline Quality Growth & .278 & .291 & -.013 & .00 & .930 \\
\hline 1 Possible to Avoid Bureau. & .488 & .500 & -.012 & .00 & .970 \\
\hline 2 Bureaucracy Definition & .250 & .229 & .021 & .01 & .940 \\
\hline 3 Opportunities to Act & .156 & .179 & -.023 & .00 & .956 \\
\hline 4 Responsibility to Act & .188 & .250 & -.062 & .08 & .775 \\
\hline 5 Example Actions & .362 & .328 & .034 & .02 & .886 \\
\hline 6 Need for Change & .208 & .358 & -.150 & .45 & .509 \\
\hline 7 Partnerships & .328 & .321 & .007 & .00 & .971 \\
\hline 8 Coaching & .225 & .143 & .082 & .15 & .705 \\
\hline
\end{tabular}

Table V shows the comparison of attitude change scores for the two sessions for the Video Control group. Although there was greater irregularity than in the Enhanced group, none of the differences were significant. Whatever differences existed in the "simulation delivery" between the August and October sessions apparently had little measurable effect on the dependent variables.

Subiect Gender. Table VI presents the results of ANOVA analysis of the mean change scores for subjects in all three treatment groups. There was no significant difference between males and females for the Quality Growth variable. Females showed greater change than males for most of the subscale variables $(1,2,4,5,6 \& 8)$ though only one of these reached significance. In light of reports that females have measured higher on some empathy scales (Stotland et al., 1978), the tendency toward greater attitude change for 
females led to speculation about the distribution of empathy scores. The mean score on the Davis "Perspective Taking" scale for females was 5.34, for males $5.29(\mathrm{~F}=.053)$.

TABLE V

COMPARISON OF SESSIONS 1 AND 2: VIDEO CONTROL

\begin{tabular}{|c|c|c|c|c|c|}
\hline Dependent Variables & $\begin{array}{l}\text { Aug. } \\
\mathrm{N}=14\end{array}$ & $\begin{array}{c}\text { Oct. } \\
\mathrm{N}=15\end{array}$ & $\begin{array}{l}\text { Aug. } \\
\text { - Oct }\end{array}$ & $\mathbf{F}$ & $\mathrm{P}$ \\
\hline Quality Growth & .327 & .330 & -.003 & .00 & .987 \\
\hline 1 Possible to Avoid Bureau. & .329 & .520 & -.191 & .17 & .684 \\
\hline 2 Bureaucracy Definition & .371 & .133 & .238 & 1.01 & .305 \\
\hline 3 Opportunities to Act & .268 & .417 & -.183 & .10 & .755 \\
\hline 4 Responsibility to Act & .446 & .083 & .363 & 1.66 & .208 \\
\hline 5 Example Actions & .459 & .353 & .106 & .10 & .752 \\
\hline 6 Need for Change & .499 & .389 & .110 & .22 & .645 \\
\hline 7 Partnerships & .089 & .500 & -.411 & 2.26 & .144 \\
\hline 8 Coaching & .029 & .227 & -.198 & 1.17 & .288 \\
\hline
\end{tabular}

TABLE VI

ANOVA COMPARISON OF ATTITUDE CHANGE BY GENDER

\begin{tabular}{|c|c|c|c|c|c|}
\hline Dependent Variables & $\begin{array}{l}\text { Male } \\
\mathrm{N}=64\end{array}$ & $\begin{array}{c}\text { Female } \\
\mathrm{N}=28 \\
\end{array}$ & $\mathrm{~F}-\mathrm{M}$ & $\mathrm{F}$ & $\underline{P}$ \\
\hline Quality Growth & .350 & .434 & .084 & 67 & .415 \\
\hline 1 Possible to Avoid Bureau. & .422 & .536 & .114 & .25 & .620 \\
\hline 2 Bureaucracy Definition & .247 & .393 & 146 & 67 & .416 \\
\hline 3 Opportunities to Act & .398 & -.125 & -.523 & 4.27 & $.041^{\circ}$ \\
\hline 4 Responsibility to Act & .242 & .574 & .332 & 5.31 & $.024^{\circ}$ \\
\hline 5 Example Actions & .456 & .704 & .248 & 2.02 & .159 \\
\hline 6 Need for Change & .406 & 631 & .225 & 2.61 & .110 \\
\hline 7 Partnerships & .332 & .278 & -.054 & .14 & .708 \\
\hline 8 Coaching & .115 & .300 & .185 & 2.91 & .092 \\
\hline - $p<$ & & & & & \\
\hline
\end{tabular}


Of interest is the one variable for which males had significantly greater change, "Opportunities to Act". A finding that it is more difficult to convince females in this organization that they have opportunities in a domain where males demonstrate significant change could lead to speculation about the role of women in the organizational environment. The pattern observed is consistent with an interpretation that some event in the organization caused a decline in females perception of "Opportunities" and that the simulation counter-acted that event.

Figure 33 indicates that subject gender was not uniformly distributed across the four groups. The Simplified Simulation group had a high proportion of females $(\mathrm{N}=18,50 \%)$ while the other groups had male-female ratios more representative of the organization $($ Control $=10 / 31=24 \%$; ES $=$ $7 / 23=30 \% ; \mathrm{VC}=4 / 25=16 \%$ ). Subject gender is a factor to consider in treatment group comparisons.

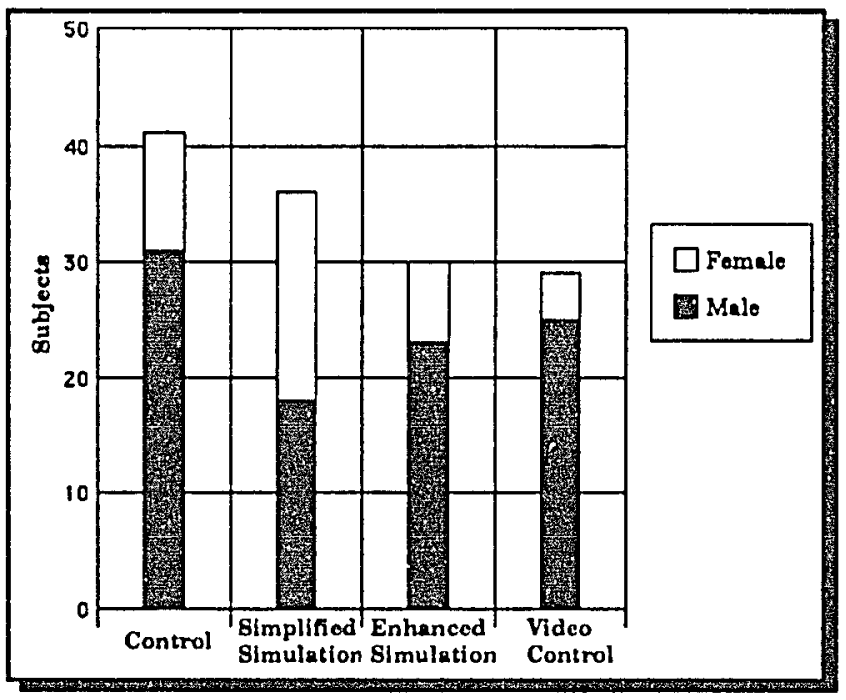

Figure 33. Treatment group gender distribution. 
Subject Age. Subject age in this study ranged from 20 to 58 (mean 37.2, SD 6.9) and was much less of a concern. Simple regression analysis produced the results in Table VII. It was not expected that age would be a significant factor in predicting attitude change, and that was the case for the Quality Growth variable. For two of the subscale variables, older subjects showed less change. Age was distributed evenly across treatment groups, means of 36.4, 39.3 and 36.2 were recorded for the Simplified Simulation, Enhanced Simulation and Video Control groups respectively. A simple regression indicated no relationship between subject age and length of employment, $\left(\mathrm{r}^{2}\right.$ $.005, \mathrm{~F}=.26)$

TABLE VII REGRESSION OF ATTITUDE CHANGE ON AGE

\begin{tabular}{|lcrc|}
\hline \multicolumn{1}{|c}{ Dependent Variables } & R-squared & $\mathrm{F}$ & $\mathrm{P}$ \\
\hline Quality Growth & .050 & 3.79 & .055 \\
1 Possible to Avoid Bureau. & .002 & .14 & .713 \\
2 Bureaucracy Definition & .024 & 1.79 & .185 \\
3 Opportunities to Act & .003 & .25 & .617 \\
4 Responsibility to Act & .030 & 2.23 & .140 \\
5 Example Actions & .127 & 10.47 & $.001 \bullet$ \\
6 Need for Change & .068 & 5.33 & $.024 \bullet$ \\
7 Partnerships & .028 & 2.04 & .157 \\
8 Coaching & .001 & .02 & .902 \\
$-p<.05$ & & & \\
\hline
\end{tabular}

Length of Employment. The length of employment in the client organization was considered a factor that could possibly influence attitude change. Table VIII shows that length of employment was a significant 
predictor of attitude change for the Quality Growth variable though the amount of variance it accounts for is small.

Conflicting hypotheses about the nature of this relationship can be generated. An obvious view is that subjects with shorter employment periods could be more actively adjusting to the organization and therefore be more open to change. Consistent with this, those with longer employment periods could be more set in their perception of this organization and less likely to change. A contrasting hypothesis would be that because the simulation calls for employees to embrace ideals more vivid in the organization's earlier days, (as argued in the ethnographic description), employees with longer employment may show greater change because the simulation appeals to their greater relevant experience. The Iatier explanation is supported as subjects with longer employment showed greater change. This supports the claim that the Quality Growth frame of reference portrays ideals common in the organization at the time of the interviews.

TABLE VIII

REGRESSION OF ATTITUDE CHANGE ON EMPLOYMENT LENGTH

\begin{tabular}{|lccl|}
\hline \multicolumn{1}{|c}{ Dependent Variables } & R-squared & $\mathrm{F}$ & $\mathrm{P}$ \\
\hline Quality Growth & .057 & 5.40 & $.022 \bullet$ \\
1 Possible to Avoid Bureau. & .039 & 3.74 & .056 \\
2 Bureaucracy Definition & .003 & .25 & .618 \\
3 Opportunities to Act & .055 & 5.33 & $.023 \bullet$ \\
4 Responsibility to Act & .002 & .17 & .675 \\
5 Example Actions & .044 & 4.06 & $.047 \bullet$ \\
6 Need for Change & .000 & .01 & .899 \\
7 Partnerships & .013 & 1.16 & .284 \\
8 Coaching & .030 & 2.87 & .094 \\
$-p<.05$ & & & \\
\hline
\end{tabular}


The mean length of employment for the three treatment groups was 44.1 months ( $\mathrm{SD}=25.3 ; \mathrm{N}=94$ ). Figure 34 shows two clusters of employment length at about 24 months and 65 months reflecting past growth spurts.

Figure 35 shows that the distribution of length of employment across the treatment groups, though not as skewed as gender, is not equal. The difference between the Simplified and Enhanced simulation groups narrowly misses significance $(p=.06)$

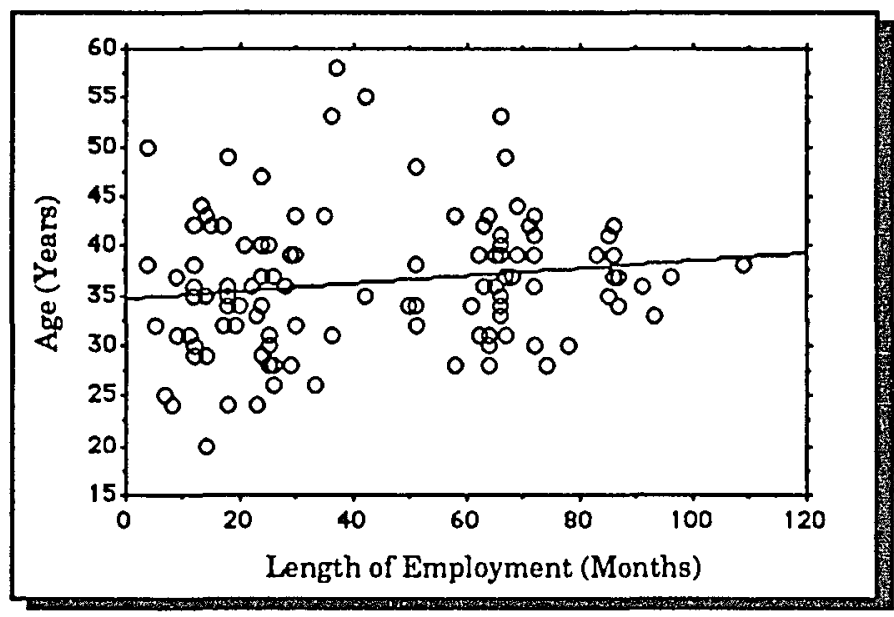

Figure 34. Subject length of employment and age.

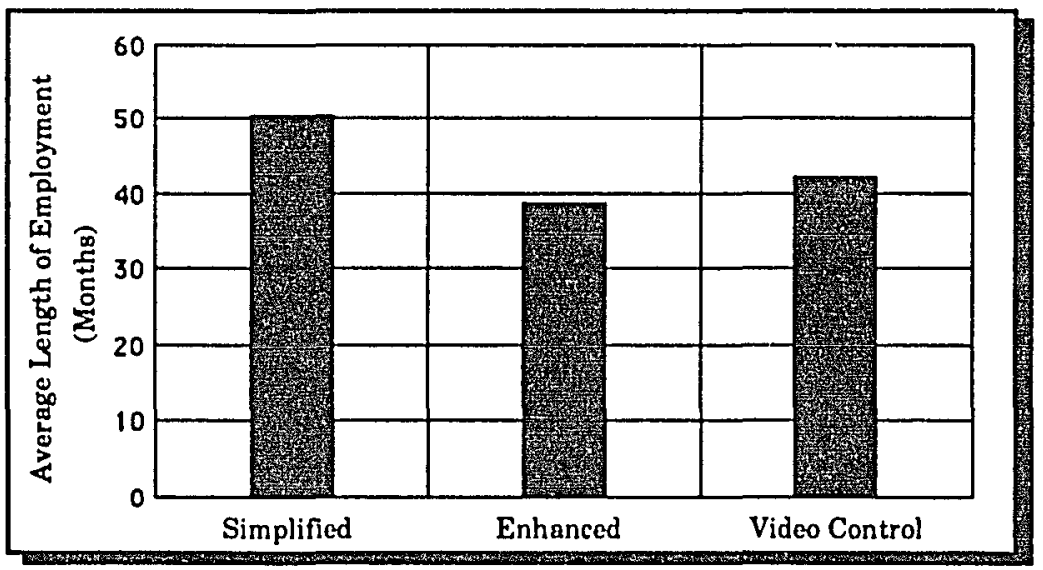

Figure 35. Subject length of employment by treatment group. 


\section{Research Question 2}

Can simulation experience cause attitude change?

H1: Is the post-simulation attitude score significantly greater than the pre-simulation score for (dependent variable $\mathrm{X}$ )?

Summary of Findings for Research Question 2. All treatment groups had significant attitude change for the Quality Growth variable while the Control group had almost none. The conclusion is that the WADA simulation caused attitude change. Graphic representations of attitude change for combined treatment and Control groups are found in Figures 37, 38, and 39 which are discussed under Research Question 7.

General Discussion of Findings for Research Question 2. This Research Question compares subjects within treatment groups on their attitudes as measured with the pre and post-simulation questionnaire. Tables IX to XII display data on change for the attitude measurements for the Control, Simplified Simulation, Enhanced Simulation and Video Control groups.

Control Group Measurement. Tables I and II indicate acceptable levels of reliability for the Quality Growth variable and the eight subscales. The data in Table IX shows that there was essentially no change for the Control Group in the Quality Growth measure. For subscale variables D6 and D7 there was change beyond the .05 level. The content of dependent variable D6, "Need for Change" makes it susceptible to many types of organizational events.

Two possible sources for Control group change have been identified. Several participants who were contacted after the study indicated that they had discussed their simulation experience in staff meetings because of its perceived value. One had taken the simulation booklets to distribute to 
While these events may or may not reflect an impact that the use of the simulation had on the organization, their measurable effect during data collection for this study seems at most to have influenced attitudes about "Need for Change" (D6) and "Partnerships" (D7). Considering that five months elapsed between pre and post-simulation measurements for the Control group, this represents minor change.

Table X displays the Simplified Simulation treatment group data and indicates there was change well beyond the .01 level. Subscale variable 'D3' was the only one without significant change. This was the variable for which females had significantly less change than males (Table VI). The Simplified Simulation group was $50 \%$ female (Figure 33 ).

TABLE X

SIMPLIFIED SIMULATION GROUP SCORES (N=37)

\begin{tabular}{|lccccc|}
\hline \multicolumn{1}{|c}{ Dependent Variables } & Pre & Post & Change & $\mathrm{t}^{*}$ & $\mathrm{P}$ \\
\hline Quality Growth & 5.00 & 5.52 & .514 & 3.154 & $.0016^{\bullet}$ \\
1 Possible to Avoid Bureau. & 3.62 & 4.06 & .434 & 2.909 & $.0031 \bullet$ \\
2 Bureaucracy Definition & 5.27 & 5.73 & .400 & 2.493 & $.0088 \bullet$ \\
3 Opportunities to Act & 4.62 & 4.79 & .176 & 0.877 & .1932 \\
4 Responsibility to Act & 5.59 & 6.18 & .557 & 5.354 & $.0001 \bullet$ \\
5 Example Actions & 4.86 & 5.61 & .783 & 6.376 & $.0001 \bullet$ \\
6 Need for Change & 5.49 & 6.16 & .673 & 7.030 & $.0001 \bullet$ \\
7 Partnerships & 5.82 & 6.15 & .338 & 3.159 & $.0017 \bullet$ \\
8 Coaching & 5.94 & 6.26 & .324 & 2.460 & $.0093 \bullet$ \\
P & & & & & \\
P One tailed paired t-Test. & & & & & \\
\hline
\end{tabular}

Tables XI and XII indicate that there was significant change in the Quality Growth variable for the Enhanced Simulation and the Video Control groups. For all dependent variables, the mean scores in all treatment groups 
did change in the direction predicted, whereas in the Control group three of the eight subscales measured change in the opposite direction.

TABLE XI

ENHANCED SIMULATION GROUP SCORES (N=30).

\begin{tabular}{|c|c|c|c|c|c|}
\hline Dependent Variables & Pre & Post & Change & $t^{*}$ & $\mathbf{P}$ \\
\hline Quality Growth & 5.37 & 5.66 & .288 & 3.182 & $.0018^{\circ}$ \\
\hline $\begin{array}{l}1 \text { Possible to Avoid Bureau. } \\
2 \text { Bureaucracy Definition } \\
3 \text { Opportunities to Act } \\
4 \text { Responsibility to Act } \\
5 \text { Example Actions } \\
6 \text { Need for Change } \\
7 \text { Partnerships } \\
8 \text { Coaching }\end{array}$ & $\begin{array}{l}3.80 \\
5.65 \\
4.94 \\
5.93 \\
5.39 \\
5.55 \\
5.74 \\
5.91\end{array}$ & $\begin{array}{l}4.30 \\
5.89 \\
5.11 \\
6.15 \\
5.77 \\
5.78 \\
6.07 \\
6.06\end{array}$ & $\begin{array}{l}.493 \\
.240 \\
.167 \\
.217 \\
.347 \\
.278 \\
.325 \\
.147\end{array}$ & $\begin{array}{l}3.044 \\
1.746 \\
0.874 \\
2.037 \\
3.050 \\
2.513 \\
3.651 \\
1.302\end{array}$ & $\begin{array}{l}.0025^{\circ} \\
.0456 \\
.1948^{\circ} \\
.0254 \\
.0026^{\bullet} \\
.0089^{\bullet} \\
.0005^{\bullet} \\
.1016^{\circ}\end{array}$ \\
\hline
\end{tabular}

TABLE XII

VIDEO CONTROL GROUP SCORES ( $\mathrm{N}=28)$

\begin{tabular}{|lccccc|}
\hline \multicolumn{1}{|c}{ Dependent Variables } & Pre & Post & Change & t* & P \\
\hline Quality Growth & 5.33 & 5.66 & .328 & 3.485 & $.0008 \bullet$ \\
1 Possible to Avoid Bureau. & 3.85 & 4.28 & .429 & 1.807 & .0410 \\
2 Bureaucracy Definition & 5.59 & 5.84 & .243 & 2.060 & .0246 \\
3 Opportunities to Act & 4.76 & 5.10 & .345 & 1.485 & .0744 \\
4 Responsibility to Act & 5.84 & 6.10 & .259 & 1.818 & .0399 \\
5 Example Actions & 5.17 & 5.58 & .424 & 2.507 & $.0093 \bullet$ \\
6 Need for Change & 5.71 & 6.15 & .446 & 3.698 & $.0005 \bullet$ \\
7 Partnerships & 5.63 & 5.94 & .321 & 2.246 & .0166 \\
8 Coaching & 6.10 & 6.23 & .131 & 1.430 & .0819 \\
P P .01 & & & & & \\
One tailed paired t-Test. & & & & & \\
\end{tabular}




\section{Research Question 3}

Do certain structural attributes in the simulation increase 'Role

\section{Identification' for participants?22}

H2: Do subjects in the Enhanced Simulation group report greater Role Identification scores than those in the Simplified Simulation?

H3: Is there a significant difference between the Role Identification scores of subjects in the Enhanced Simulation group and those in the Video Control group?

Summary of Findings for Research Question 3. The different simiulation structures that defined the Enhanced Simulation group - primarily the presentation of one role versus two roles (but not the video tape) - produced significantly greater levels of role identification than found in the Simplified Simulation group. Consistent with predictions, there was no difference in levels of role identification between the Enhanced Simulation and Video Control groups.

Subjects in the Enhanced Simulation and Video Control groups perceived the Beta role as significantly more different from the Mentor Graphics' culture than did subjects in the Simplified Simulation. Research Question 3 is therefore answered affirmatively. Additional evidence to support this conclusion is presented under Research Question 4.

22 It should be noted that answering Research Question 3 did not require participants to return questionnaires as the two variables, "simulation structure" and "simulation experience", were determined during the classes. This is noteworthy because data is available for a larger number of subjects, $(N=118$ - essentially all who played the simulation) rather than just the 86 subjects reported in Tables XIII and XIV who completed pre- and post-simulation questionnaires. The smaller subset is reported here to insure a uniform subject pool with the remaining research questions. 
General Discussion of Findings for Research Question 3. This Research Question compares treatment groups on two measures of the mediating variable. The structural attributes that defined treatment groups were listed in Figure 29.

Role Identification. The mediating variable was intended to assess differences between subjects in what Bredemeier and Greenblat (1981) label "simulation experience". This research question explores whether subjects who experienced different simulation structures report differences in "simulation experience". Previous research suggested that identification with a simulation role was one dimension of simulation experience that may be important and that variable was identified in the hypotheses of this study ${ }^{23}$.

To measure subjects' identification with the Beta simulation role, the simulation software presented questions to them at the end of the Beta game. These are listed in Figure 36 along with the response options. Questions 1 through 4 assess participants' role identification, asking them the extent that they were aware of adopting the Beta frame of reference. The responses of each subject to these questions were averaged to produce a "Role ID" value. Cronbach's alpha was calculated for the combined treatment group data (Alpha $=0.755, \mathrm{~N}=86$ ). Question 4 correlated poorly with the other "Role ID" questions (all <.1) and measured little difference between the groups.

Role-Culture Contrast. The broader purpose of the mediating variable was to assess differences in participants' "simulation experience". Question 5 (Figure 36) asked participants to compare the Beta role to the Mentor

23 Another dimension, the extent to which subjects enjoyed playing the simulation, was also recorded and results are presented in Chapter IV, Discussion. 
Graphics culture, something which all subjects had experienced (mean employment was 44 months, see Figures 34 and 35). Responses to this question were used as a variable labeled "Role-Culture Contrast" (R-CC). Although only a single question was used to measure this variable, the Mentor Graphics culture was seen as a standard within the organizational reality of subjects that would provide a good basis for assessing their perception of the Beta role. That role was identical for all treatment groups and therefore different perceptions of that role reflect differences in subjects' "simulation experience".

\section{Role Identification: "Role ID"}

1. During the simulation, I felt that I was seeing the events of the simulation as a Betan:

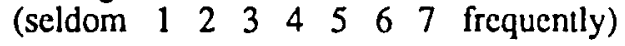

2. I believe my decisions in the Beta play of the simulation were consistent with the Beta culture: (never $1120 \begin{array}{lllllll} & 2 & 4 & 5 & 6 & 7 & \text { always) }\end{array}$

3. While playing WADA as a Betan, I considered the cultural description when making

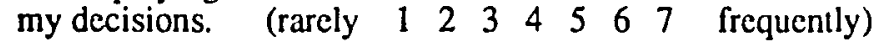

4. While playing Winning At Design Automation, I imagined that I was a Betan manager working in the Beta company: (rarely $1 \begin{array}{lllllllll} & 2 & 3 & 4 & 5 & 7 & \text { frequently) }\end{array}$

\section{Role-Culture Contrast: "R-CC"}

5. In comparing the Beta culture to the culture of the organization where I work, I would

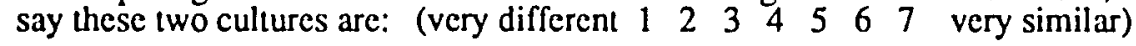

6. In comparing the Alpha culture to the culture of the organization where I work, I would

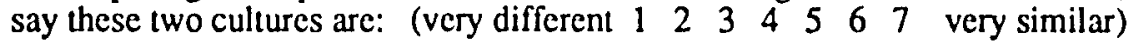

Figure 36. Mediating variable questions.

In some ways the R-CC measure is more 'objective' than the Role ID variable, as the latter is defined more completely within the realm of each subject's cognition. The Role-Culture Contrast question references 
somathing that exists external to each individual. Obviously the culture of any organization is complex and what elements of the MGC culture various subjects had experienced, or which they referenced in making their assessment of contrast, is not known. Balancing this uncertainty, the context of being asked to gauge this contrast after several hours of playing a simulation role would suggest that the aspects of the culture considered for comparison would be those represented in the role.

Although questions remain about interpretation of the Role-Culture Contrast variable, it assessed whether the different simulation versions conveyed different "simulation experience" to subjects. R-CC was not considered during the writing of the study proposal and is not included in those hypotheses. It is therefore considered only within the broader discussion of exploring this research question.

Role ID and R-CC relationship. WADA was designed with the expectation that the Enhanced Simulation group would experience both greater identification with the Beta role and a greater perception of difference between that role and the Mentor Graphics culture. The R-CC and Role ID variables were expected to have a strong negative correlation (the R-CC was reverse scored). Data collected during the simulation classes about the relationship between these variables was inconsistent for the different treatment groups. For the Simplified Simulation group only, the correlation was $.307\left(\mathrm{R}^{2}=.094, \mathrm{~N}=35\right)$ indicating that as identification with the Beta role increased, perceived contrast between that role and the MGC culture decreased (contrary to predictions). For the Enhanced Simulation, the correlation was $-.032\left(\mathrm{R}^{2}=.001, \mathrm{~N}=28\right)$. 
Role ID and R-CC were not strongly correlated (contrary to expectations). This is some support for the claim that they do indeed represent separate aspects of subjects' simulation experience. Question 6 (Figure 36) was asked at the end of the Alpha game as a measure of how well that cultural role matched the culture of the client organization. (It was not used in calculating the mediating variable because there was no measure of it for the Simplified Simulation group who played only the Beta role.) The average for the Enhanced Simulation group was 5.79 and for the Video Control group 6.07. Comparing this to the results of Question 5 in Table XIII indicates that subjects did perceive the roles as intended - the Alpha role similar to Mentor Graphics' culture, Beta different.

TABLE XIII

MEDIATING VARIABLE COMPARISON: ENHANCED-SIMPLIFIED

\begin{tabular}{|c|c|c|c|c|c|}
\hline Question & $\begin{array}{l}\text {-Group } \mathrm{Me} \\
\text { Enhanced } \\
\text { Simulation } \\
(\mathrm{N}=28)\end{array}$ & $\begin{array}{l}\text { Scores- } \\
\text { Simplified } \\
\text { Simulation } \\
(N=35)\end{array}$ & $\begin{array}{l}\text { Enhanced- } \\
\text { Simplified }\end{array}$ & or respon & options) \\
\hline $\begin{array}{l}1 \\
2 \\
3 \\
4\end{array}$ & $\begin{array}{l}6.11 \\
6.04 \\
6.64 \\
6.07\end{array}$ & $\begin{array}{l}5.74 \\
5.54 \\
6.34 \\
6.03\end{array}$ & $\begin{array}{l}.37 \\
.50 \\
.29 \\
.04\end{array}$ & $\begin{array}{r}1.69 \\
2.79 \\
1.59 \\
.03\end{array}$ & $\begin{array}{l}.0468^{\circ} \\
.0036^{\bullet} \\
.0599 \\
.4319\end{array}$ \\
\hline Role ID & 6.19 & 5.91 & .28 & 1.72 & $.0454^{\bullet}$ \\
\hline $\begin{array}{l}\text { R-CC } 5 \\
* \text { One Tailed, } \\
\cdot \mathrm{p}<.05\end{array}$ & $\begin{array}{c}2.75 \\
\text { unpaired } t \text {-tes }\end{array}$ & 3.57 & -.82 & -2.27 & $.0132 \bullet$ \\
\hline
\end{tabular}

Treatment Group Comparisons. Table XIII displays the mean scores and t-Test results for the Enhanced Simulation and Simplified Simulation 
groups. There was a significant difference between these groups for two of the four role identification questions, and the composite variable. The results for Question 5 indicate that subjects who experienced the Enhanced Simulation consider the Beta role to be more different from the Mentor Graphics culture than do subjects in the Simplified Simulation group.

Table XIV reports t-Test analysis indicating there is no significant difference between the responses of the Enhanced Simulation and Video Control groups. The video did not have a significant effect on subjects' reports of role identification or their comparison of that role to the MGC culture as predicted. Subjects in the Video Control group perceived the Beta role to be even more different than the MGC culture than those who saw the video of Mentor Graphics leaders taking about the culture. Although this difference was not significant, the video could have had an impact on subjects' assessment of the MGC culture.

TABLE XIV

MEDIATING VARIABLE COMPARISON: ENHANCED-VIDEO CONTROL

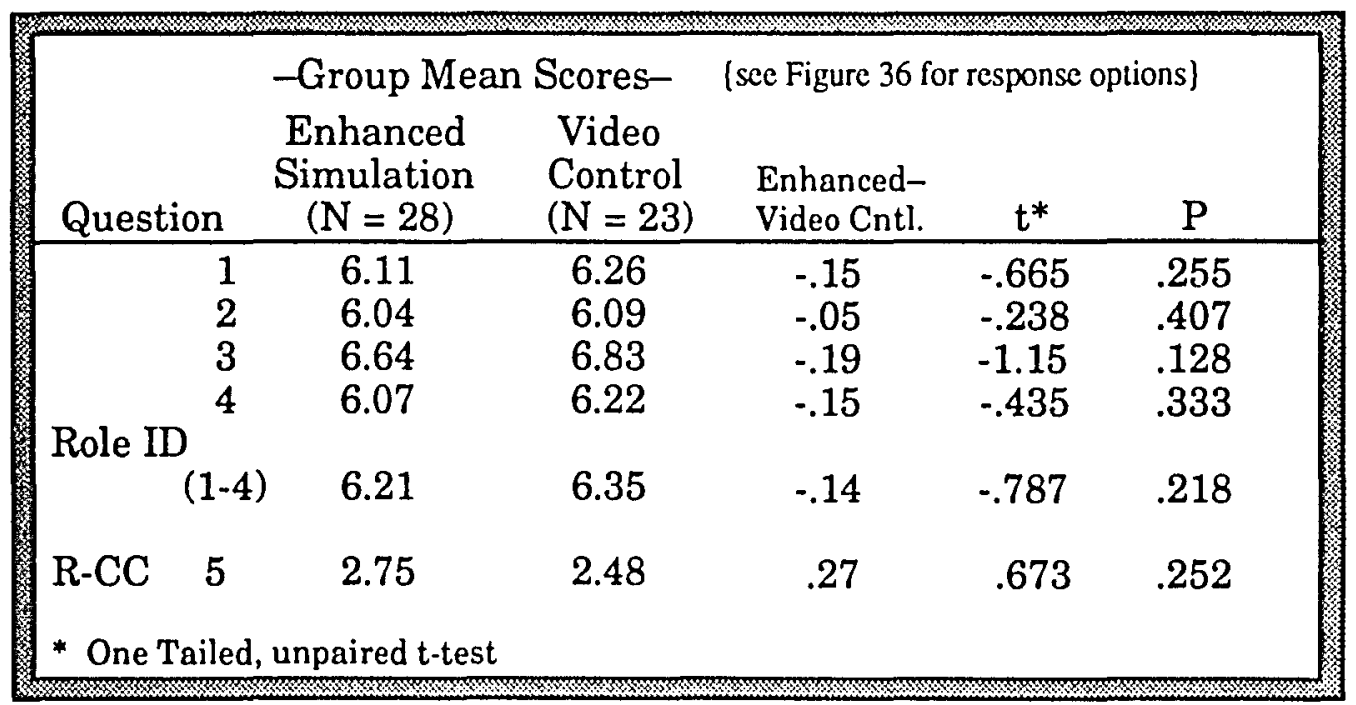




\section{Research Question 4}

Is the cognitive style of the participant, as measured by the cognitive subscales of the Interpersonal Reactivity Index, a factor predicting 'Role Identification' during a simulation experience?

H4: Do subjects who score higher on 'Perspective Taking' report greater scores on 'Role Identification'?

H5: Do subjects who score higher on 'Fantasy' report greater scores on 'Role Identification'?

Summary of Findings for Research Question 4. The "Perspective Taking" subscale of the IRI is a significant predictor of subjects' reports of role identification. The "Fantasy" subscale is not. Neither is significant as a factor predicting subjects' assessment of the difference between the Beta role and the MGC culture (Role-Culture Contrast). Research Question 4 is affirmed with the narrower conclusion that only Hypothesis $\mathrm{H} 4$ is supported. This is the hypothesis with the most theoretical support.

Consistent with the conclusions of the previous research question, treatment group is again seen to be a significant factor determining both subjects' role identification and their assessment of the contrast between the Beta-role and MGC culture.

General Discussion of Findings for Research Question 4. The two sevenitem cognitive subscales of the IRI, "Perspective Taking" and "Fantasy" were included in the questionnaire as measures of cognitive style. Subjects' average scores for these subscales serve as independent variables in answering Research Question 4. Similar to Research Question 3, the mediating variables "Role Identification" and "Role-Culture Contrast" (Figure 36) act as dependent variables. Regression models were constructed because 
the Cognitive Style, "Role Identification" and "Role-Culture Contrast" variables are continuous. Data from all three treatment groups was combined for this analysis and G1 and G2 represent dummy variables for treatment group. Table XV presents the results of the "Perspective Taking" regression models. The $\mathrm{F}$ value of 10.05 indicates that this subscale is a significant factor predicting subjects' self report of identification with the simulation role. "Perspective Taking" was not a predictor of the "RoleCulture Contrast" variable, a finding that reflects the independence of the latter from "Role ID".

\section{TABLE XV}

PERSPECTIVE TAKING REGRESSION MODELS

\begin{tabular}{|lccrc|}
\hline Model Variables* & $\mathrm{R}$ & $\mathrm{R}^{2}$ & $\mathrm{~F}$ & $\mathrm{P}$ \\
\hline Independent: & .427 & .183 & 6.11 & .0008 \\
Perspective Taking & & & 10.05 & .0021 \\
G1 (group dummy) & & & 6.48 & .0128 \\
G2 (group dummy) & & & .79 & .3757 \\
Dependent: & & & & \\
Role Identification & & & & \\
\hline Independent: & .322 & .104 & 3.16 & .0290 \\
Perspective Taking & & & .07 & .7880 \\
G1 (group dummy) & & & .92 & .0061 \\
G2 (group dummy) & & & .46 & .4995 \\
Dependent: & & & \\
Role-Culture Contrast & & & \\
* Three treatment groups, $\mathrm{N}=86$ & & & \\
\hline
\end{tabular}

Table XVI presents the results of regression models using the "Fantasy" subscale as an independent variable. The "Fantasy" subscale is not a 
significant factor predicting either subjects' "Role Identification" with the simulation role or their perception of "Role-Culture Contrast".

TABLE XVI

FANTASY REGRESSION MODELS

\begin{tabular}{|lcccc|}
\hline Model Variables* & $\mathrm{R}$ & $\mathrm{R}^{2}$ & $\mathrm{~F}$ & $\mathrm{P}$ \\
\hline Independent: & .288 & .083 & 2.47 & .0680 \\
Fantasy & & & 0.02 & .8982 \\
G1 (group dummy) & & & 6.50 & .0126 \\
G2 (group dummy) & & & .57 & .4519 \\
Dependent: & & & & \\
Role Identification & & & & \\
& .338 & .114 & 3.53 & .0180 \\
Independent: & & & 1.08 & .3029 \\
Fantasy & & & .9 .93 & .0037 \\
G1 (group dummy) & & & .4947 \\
G2 (group dummy) & & & .49 \\
Dependent: & & & \\
Role-Culture Contrast & & & \\
* Three treatment groups, $\mathrm{N}=86$ & &
\end{tabular}

\section{Research Question 5}

Is the level of subjects' 'Role Identification' a factor that predicts attitude change caused by simulation experience?

H6: Do subjects who report greater levels of 'Role Identification' have greater attitude change (for dependent variable $\mathrm{Y}$ )?

Summary of Findings for Research Question 5. The data do not support a conclusion that greater role identification leads to greater attitude change. Hypothesis $\mathrm{H} 6$ was not affirmed. 
In seeking an explanation for this finding, relationships between "RoleCulture Contrast" and attitude change were explored although the analysis and conclusions involving "Role-Culture Contrast" are speculative. R-CC had a non-linear relationship to attitude change. Subjects with mid-range RCC scores had significantly greater attitude change than those with high or low scores, a finding for which an explanatory model was offered.

General Discussion of Findings for Research Question 5. The analysis for this research question considers the relationship of the two mediating variables and attitude change. Data from the Enhanced Simulation and Simplified Simulation treatment groups was pooled in this analysis.

Role Identification. The group mean was calculated for the mediating variable "Role $\mathrm{ID}^{\text {" }}$ and subjects with scores above the mean were assigned to a "high" identification group. Subjects with scores below the mean were assigned to a "low" identification group. Table XVII shows the results of tTest analysis indicating there were no significant differences in attitude change between these groups. Consistent with predictions, for the Quality Growth variable and six of the eight subscale variables, the "high" identification group change scores were greater than those of the "low" group.

This absence of any significant relationship between role identification and attitude change is counter to the conclusions of previous researchers and the causal model adopted in this study. It was decided to examine relationships involving the Role-Culture Contrast variable in a search for an explanation of this unexpected finding. It is noted that the analysis in the following section is considered very tentative because the "Role-Culture Contrast" variable does not have either as strong a theoretical backing or as robust an empirical measurement as the "Role ID" variable. 
Role-Culture Contrast. It was the intent of the simulation design that the Enhanced Simulation would create a perception of greater contrast between the fictional Beta culture and the Mentor Graphics culture at the same time it created greater role identification with that role. The previous Research Question reported the success in achieving this goal. It was expected that the relationship of " $\mathrm{R}-\mathrm{CC}^{n}$ to attitude change would be linear and positive, greater perceived contrast leading to greater change.

TABLE XVII

ROLE IDENTIFICATION AND ATTITUDE CHANGE

\begin{tabular}{|c|c|c|c|c|c|}
\hline Dependent Variables & $\begin{array}{c}\text { RoleIden } \\
\text { "high" } \\
\text { N=28 }\end{array}$ & $\begin{array}{l}\text { ification } \\
\text { "low" } \\
\mathrm{N}=33\end{array}$ & $\mathrm{H}-\mathrm{L}$ & $t^{*}$ & $\mathbf{P}$ \\
\hline Quality Growth & .444 & .388 & .056 & .517 & .304 \\
\hline $\begin{array}{l}1 \text { Possible to Avoid Bureau. } \\
2 \text { Bureaucracy Definition } \\
3 \text { Opportunities to Act } \\
4 \text { Responsibility to Act } \\
5 \text { Example Actions } \\
6 \text { Need for Change } \\
7 \text { Partnerships } \\
8 \text { Coaching }\end{array}$ & $\begin{array}{l}.450 \\
.471 \\
0.000 \\
.457 \\
.709 \\
.608 \\
.375 \\
.214\end{array}$ & $\begin{array}{l}.521 \\
.218 \\
.382 \\
.328 \\
.530 \\
.445 \\
.318 \\
.200\end{array}$ & $\begin{array}{r}. .071 \\
.253 \\
-.382 \\
.129 \\
.179 \\
.163 \\
.057 \\
.014\end{array}$ & $\begin{array}{r}-.306 \\
1.159 \\
-1.384 \\
.819 \\
.961 \\
1.028 \\
.387 \\
.110\end{array}$ & $\begin{array}{l}.380 \\
.126 \\
.086 \\
.208 \\
.170 \\
.154 \\
.350 \\
.456\end{array}$ \\
\hline \multicolumn{6}{|c|}{$\begin{array}{l}\text { - Combined treatment groups (N=61) } \\
\text { * One tailed unpaired t-Test }\end{array}$} \\
\hline
\end{tabular}

In contrast to these expectations, exploratory analysis indicated that the relationship of "Role-Culture Contrast" to attitude change was non-linear. Table XVIII displays the results of second degree polynomial regression models with $\mathrm{R}-\mathrm{CC}$ the independent variable. This data suggests that the initial expectation was too simplistic - there was little attitude change with both very low and very high levels of perceived Role-Culture contrast. 
TABLE XVIII

ROLE CULTURE CONTRAST REGRESSION MODELS

\begin{tabular}{|lcccc|}
\hline Dependent Variables* & $\mathrm{R}$ & $\mathrm{R}^{2}$ & $\mathrm{~F}$ & $\mathrm{P}$ \\
\hline Quality Growth & .472 & .222 & 8.30 & .0007 \\
1 Possible to Avoid Bureau. & .360 & .129 & 4.31 & .0180 \\
2 Bureaucracy Definition & .349 & .122 & 4.03 & .0229 \\
3 Opportunities to Act & .233 & .054 & 1.72 & .1874 \\
4 Responsibility to Act & .467 & .218 & 8.10 & .0008 \\
5 Example Actions & .487 & .237 & 8.70 & .0005 \\
6 Need for Change & .341 & .117 & 3.83 & .0275 \\
7 Partnerships & .109 & .012 & 0.35 & .7091 \\
8 Coaching & .091 & .008 & 0.25 & .7990 \\
* Second order polynomial regression, independent variable: $\mathrm{R}-\mathrm{CC}$ & \\
Enhanced and Simplified treatment groups, (N =61) & &
\end{tabular}

A theoretical explanation for a non-linear model of $\mathrm{R}-\mathrm{CC}$ effect exists. For subjects who perceive the simulation ideal to be similar to the existing MGC culture there is little motivation for them to change their attitudes when they are presented with that ideal - they perceive the MGC culture as already similar to the Beta role. At greater levels of perceived contrast there is greater dissonance - and greater motivation for subjects to change - when they attempt to assimilate the ideal role. At more extreme levels of contrast, the dissonance is too great for easy assimilation - the Beta ideal begins to be rejected as irrelevant, as not credible, as being beyond reach, etc. Beyond that point subjects no longer attempt to assimilate the contrasting ideal and motivation to change declines. For subjects who perceive the contrast to be this extreme, the Beta cultural role is outside the bounds of acceptance.

The initial expectation of a linear increase in attitude change with increasing perceived Role-Culture Contrast carried the implicit assumption 
that the careful design of the Beta role would keep it within the lattitude of acceptance for all subjects in all treatment conditions. Table XVIII indicates that this assumption was wrong.

The non-linear relationship suggested by Table XVIII indicated that a test for significant differences should divide subjects into "high", "medium" and "low" groups based on their R-CC scores. The R-CC was recorded on a seven point scale and the mode was 3 (mean of 3.2). The "medium" group was defined as subjects with the mode score and those one point above and below the mode. The "high" and "low" groups were combined to perform t-Test analysis. Table XIX reports the mean change scores for these groups and indicates that the "medium" group did have significantly greater change for the Quality Growth variable, the primary indicator of attitudes.

\section{TABLE XIX}

ROLE CULTURE CONTRAST AND ATTITUDE CHANGE

\begin{tabular}{|c|c|c|c|c|c|}
\hline Dependent Variables & $\begin{array}{l}\text { Role-C } \\
\text { "Med." } \\
\mathrm{N}=38\end{array}$ & $\begin{array}{l}\text { lture } \mathrm{Cc} \\
\text { "H+L" } \\
\mathrm{N}=23\end{array}$ & $\begin{array}{l}\text { ntrast } \\
\text { Med. } \\
-\mathrm{H}+\mathrm{L}\end{array}$ & $t^{*}$ & $P$ \\
\hline Quality Growth & .540 & .206 & .334 & 3.27 & $.0018^{\circ}$ \\
\hline $\begin{array}{l}1 \text { Possible to Avoid Bureau. } \\
2 \text { Bureaucracy Definition } \\
3 \text { Opportunities to Act } \\
4 \text { Responsibility to Act } \\
5 \text { Example Actions } \\
6 \text { Need for Change } \\
7 \text { Partnerships } \\
8 \text { Coaching }\end{array}$ & $\begin{array}{l}.732 \\
.484 \\
.316 \\
.474 \\
.821 \\
.568 \\
.355 \\
.242\end{array}$ & $\begin{array}{l}.087 \\
.087 \\
.040 \\
.250 \\
.292 \\
.445 \\
.326 \\
.152\end{array}$ & $\begin{array}{l}.645 \\
.397 \\
.276 \\
.224 \\
.529 \\
.123 \\
.029 \\
.090\end{array}$ & $\begin{array}{r}2.88 \\
1.80 \\
.97 \\
1.40 \\
2.96 \\
.76 \\
.19 \\
.71\end{array}$ & $\begin{array}{l}.0056^{\bullet} \\
.0778 \\
.3349 \\
.1681 \\
.0044^{\bullet} \\
.4507 \\
.8474 \\
.4807\end{array}$ \\
\hline \multicolumn{6}{|c|}{$\begin{array}{l}\text { - Combined treatment groups }(\mathrm{N}=61) \text {. See text for discussion of "Med.", " } \mathrm{H}+\mathrm{L}^{\text {" }} \\
\text { * Two tailed unpaired } \mathrm{t}-\mathrm{Test} \\
\text { - } \mathrm{p}<.01\end{array}$} \\
\hline
\end{tabular}




\section{Research Question 6}

Does including certain structural attributes in the simulation design enhance its ability produce attitude change beyond that governed by the mediating variables?

H7: Is the change for (dependent variable X) in the Enhanced Simulation group significantly greater than the change for the Simplified Simulation?

Summary of Findings for Research Question 6. Table XX shows that Simplified Simulation group had greater attitude change than the Enhanced Simulation group for Quality Growth and seven of the eight subscale measures. Research question six is therefore answered "No", the Enhanced Simulation did not create greater attitude change.

TABLE XX

MAGNITUDE OF CHANGE COMPARISON (ES VS SS)

\begin{tabular}{|lccc|}
\hline Dependent Variables & Simplified & Enhanced & S/E ratio \\
\hline Quality Growth & .514 & .288 & 1.78 \\
1 Possible to Avoid Bureau. & .434 & .493 & 0.88 \\
2 Bureaucracy Definition & .400 & .240 & 1.67 \\
3 Opportunities to Act & .236 & .167 & 1.41 \\
4 Responsibility to Act & .557 & .217 & 2.57 \\
5 Example Actions & .784 & .346 & 2.26 \\
6 Need for Change & .672 & .278 & 2.42 \\
7 Partnerships & .338 & .325 & 1.04 \\
8 Coaching & .211 & .187 & 1.13 \\
\hline
\end{tabular}

General Discussion of Findings for Research Question 6. This research question asks whether the additional simulation structures which defined the Enhanced Simulation treatment condition produced greater change in 
subjects' attitudes than did the Simplified Simulation. The intent was to uncover any differential effect for the simulation that was separate from the mediating variables. Table XX presents the mean change scores for each variable from tables $\mathrm{X}$ and $\mathrm{XI}$. The magnitude of change in the Simplified Simulation group was greater than in the Enhanced Simulation group.

\section{Research Question 7}

Are there any "system-level" effects (enduring frame of reference change) accompanying simulation-induced attitude change?

Summary of Findings for Research Question 7. Together the evidence of the indicators support the conclusion that some system-level effect occurred. Subjects, at least to some limited degree, integrated the Beta cultural role into their organizational realities. They learned a system of attitudes and beliefs that could be labeled a schema or frame of reference. Research question seven is answered affirmatively.

General Discussion of Findings for Research Question 7. The "systemlevel" model of change discussed within the Constructivist literature suggested that simulation experience may reduce the "systemic resilience" to attitude change by inducing change at a more global level, by supporting participants in adopting new frames of reference. Research Question 7 was not intended to prove the existence of "system-level" change, but only to determine if there was any evidence to support this idea. Four indicators of "system-level" change in subjects' organizational cognition were identified at the beginning of the study. These are analyzed separately. There were no hypotheses associated with Research Question 7.

Capstene Variable Change. The study proposal assumed that the new frame of reference to be designed in this study would have a hierarchical 
structure, consistent with widely held beliefs in the schema literature. The apex of that structure, later termed the "capstone variable", would be more abstract and broader in scope than lower levels of the hierarchy. Because it measured change in the broadest domain addressed by the simulation, the demonstration of change for this variable could indicate that the Beta frame of reference had been incorporated into subjects' mental maps. Had there been no change in this variable, it would have been a very strong claim for no such "system-level" change.

Table XXI reproduces the t-Test data from previous tables on the capstone variable "Possible to Avoid Bureaucracy". The magnitude of change was similar for each treatment group and this was significant beyond the .01 level for two of the three groups. The mean score for each treatment group moved across the scale mid-line (4.0), from "slightly disagree" to "slightly agree". The capstone variable indicator of change supports the claim that some "system-level" change occurred.

TABLE XXI

\section{COMPARISON OF CAPSTONE VARIABLE CHANGE}

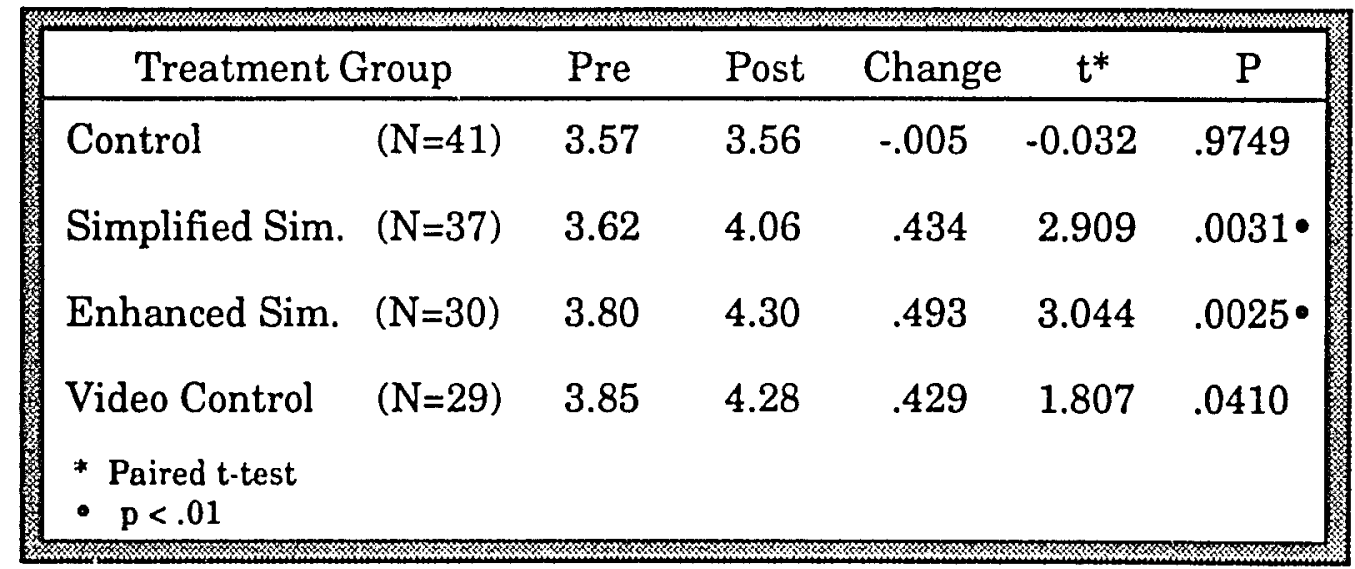


Uniformity of Attitude Change. This indicator is weaker than the first because its validity is closely linked to assumptions about relationships between the subscale variables. The study developed little in the way of measurements to assess those relationships

If there were attitude scales for which change was expected but no change occurred, or if some scales changed in the direction opposite to predictions, then claims that that there was "system-level" change would be weakened. (Differential expectations of change were discussed in the first section of this chapter.)

Table $\mathrm{X}$ indicates that the Simplified Simulation version created significant change for "Quality Growth" and seven of the eight subscales. The variable for which there was no significant change within the treatment groups (D3 "Opportunities"), was seen to be related to subject gender. There were no variables for which the group mean changed in the direction opposite than predicted in the treatment groups, as did occur in the Control group. The breadth of change observed in the Simplified Simulation group supports the claim that some "system-level" change occurred.

Subscale Variable Correlation Change. It was reasoned that if subjects learned a system of cognitive elements, they would then consider the elements of that system to be more correlated. Tables XXII and XXIII display the pre and post-simulation correlation matrices of the eight dependent variables for the combined treatment groups. Table XXIV shows the average correlations for the Control and combined treatment groups.

There was a slight change toward greater correlation for the Control group while a much larger change toward greater correlation was observed 
for the treatment groups. This indicator supports the claim that some

"system-level" change occurred.

TABLE XXII

PRE SIMULATION DEPENDENT VARIABLE CORRELATION MATRIX

\begin{tabular}{|c|c|c|c|c|c|c|c|c|}
\hline & D 1 & D 2 & D 3 & D 4 & D 5 & D 6 & D 7 & D 8 \\
\hline \multicolumn{9}{|c|}{ D $1 \longdiv { 1 }$} \\
\hline D 2 & -.017 & 1 & & & & & & \\
\hline D 3 & .424 & -.012 & 1 & & & & & \\
\hline D 4 & .177 & .476 & .292 & 1 & & & & \\
\hline D 5 & .176 & .542 & .384 & .553 & 1 & & & \\
\hline D 6 & .027 & .356 & .047 & .428 & .390 & 1 & & \\
\hline D 7 & .115 & .067 & .270 & .136 & .257 & .285 & 1 & \\
\hline D 8 & \begin{tabular}{|l|}
.275 \\
\end{tabular} & .320 & .235 & .463 & .280 & .343 & .476 & 1 \\
\hline \multicolumn{9}{|c|}{ Average $=.277$} \\
\hline
\end{tabular}

TABLE XXIII

POST SIMULATION DEPENDENT VARIABLE CORRELATION MATRIX

\begin{tabular}{|c|c|c|c|c|c|c|c|c|}
\hline & D 1 & D 2 & D 3 & D 4 & D 5 & D 6 & D 7 & D 8 \\
\hline \multicolumn{9}{|c|}{ D $1 \longdiv { 1 }$} \\
\hline D 2 & .039 & 1 & & & & & & \\
\hline D 3 & .334 & .099 & 1 & & & & & \\
\hline$D_{4}$ & .393 & .493 & .357 & 1 & & & & \\
\hline D 5 & .317 & .598 & .455 & .728 & 1 & & & \\
\hline D 6 & .384 & .467 & .245 & .634 & .658 & 1 & & \\
\hline D 7 & .218 & .174 & .357 & .425 & .531 & .405 & 1 & \\
\hline D 8 & .269 & .369 & .196 & .510 & .504 & .590 & .462 & 1 \\
\hline \multicolumn{9}{|c|}{ Average $=.400 \quad$ Alpha $=.842$} \\
\hline
\end{tabular}


TABLE XXIV

AVERAGE DEPENDENT VARIABLE CORRELATIONS

\begin{tabular}{|lcc|}
\hline Group & Pre-Simulation & Post-simulation \\
\hline Control (N=41) & 0.272 & 0.314 \\
Combined Treatment $(\mathrm{N}=92)$ & 0.277 & 0.400 \\
\hline
\end{tabular}

Bi-modal Change Pattern. Williams (1987) has speculated that a "threshold effect" may be associated with role identification. By this he meant that up to some critical level of identification there is little effect and above that level a substantial effect. If this were true, then in a population of subjects, it may be possible to observe some subjects with substantial attitude change and others with little or no change. A bi-modal pattern of change could indicate that some "system level" effected had occurred.

Williams' speculation on a threshold could be incorrect or it may be unrelated to a phenomena such as "system-level" change. Subjects' role identification with the Beta culture could all (or none) be above the "threshold", or the difference in effect magnitude may be small relative to the response range attributed to the attitude scales in this study thereby blurring any tendency toward a bi-modal distribution. Accordingly, this indicator is considered the weakest of the four.

Figures 37, 38 and 39 display the distribution of subjects' change scores by attitude scale for all treatment groups. None of the distributions is clearly bi-modal. The claim that some "system-level" change occurred is not supported. 


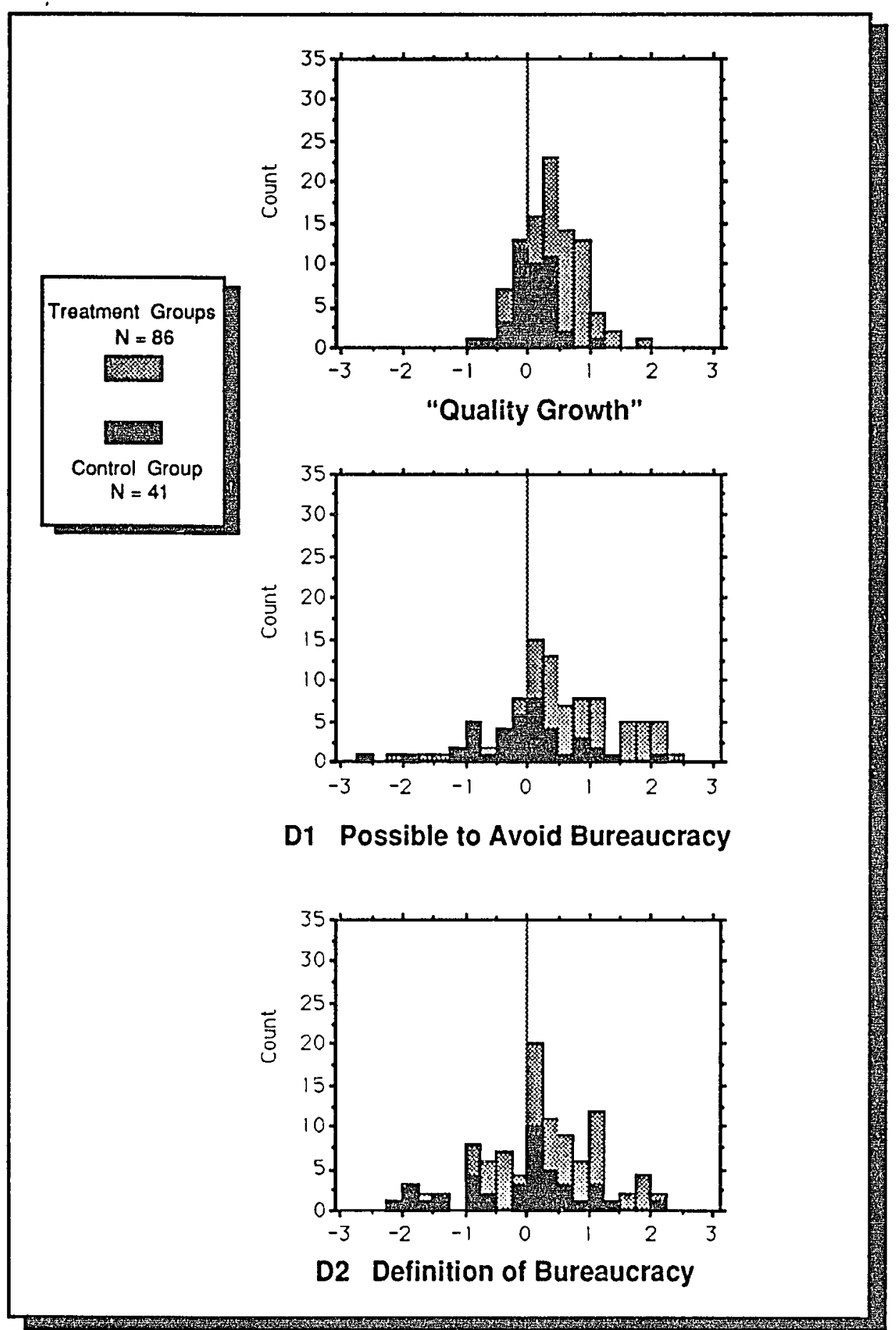

Figure 37 Magnitude of attitude change distributions. 


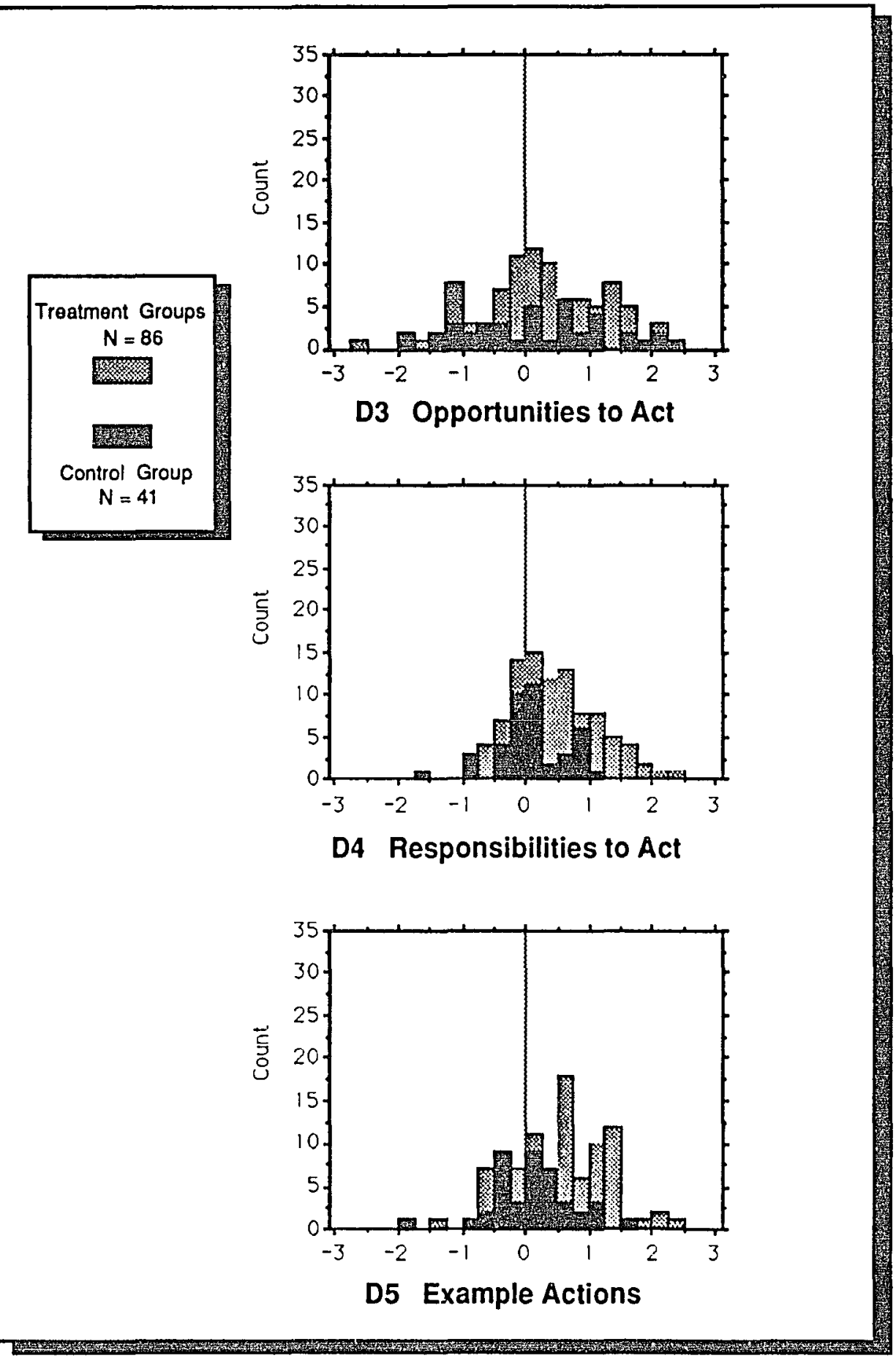

Figure 38. Magnitude of attitude change distributions (continued). 


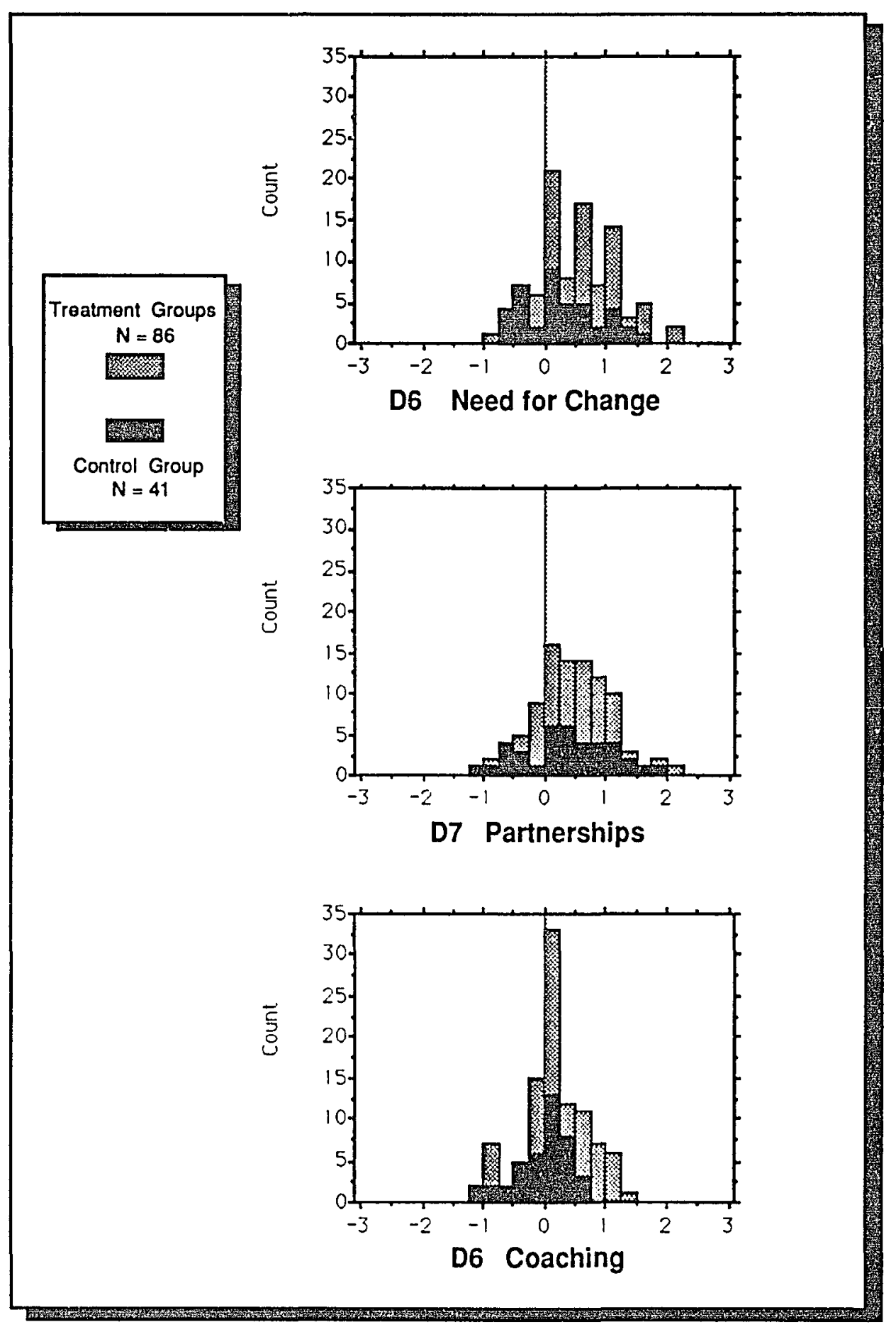

Figure 39. Magnitude of attitude change distributions (concluded). 
Participant Response. Finally, comments by participants during the classes also support the conclusion that participants' integrated the frame of reference in the simulation role with their world view. For example, many participants wanted to take their hats with them at the end of the class and one commented during the debriefing that, "We should all wear these hats every Friday to remind us of the importance of the Beta culture". People who experienced the simulation have been observed to make statements such as, "We need to be more like Beta" during meetings in the organization months after the simulation classes were held. Statements such as this, while not meeting the standards associated with scientific findings, do provide evidence that participants related to the simulation experience as a gestalt level (corresponding to the simulation role).

\section{Other Questionnaire Items}

The above analysis focused on the 40 questionnaire items that comprised the dependent variables. An additional 14 items were from the Davis Empathy scale and were used to calculate the Cognitive Style indicators used in Research Question 4. The remaining questionnaire items were included 1) to gather information for the ethnography, 2) to buttress claims about the Quality Growth frame of reference, or 3) as 'filler' items. For the items in these latter groups, no change was expected. Instead it was the raw scores that were of interest. All items were scored on a seven point scale from "strongly disagree" (1) through "neither agree or disagree" (4) to "strongly agree" (7). The following figures include responses from all groups $(N=135)$.

Four items were combined into a "rewards" variable that was not targeted for change. It was recorded only to present the raw scores to Mentor Graphics' management as an indication of the perception of organizational 
Graphics' management as an indication of the perception of organizational support for the behaviors the simulation suggests require change. The items are shown in Figure 40 along with descriptive statistics of the pre-simulation scores for all groups.

2. Mentor Graphics rewards managers who do a good job of coaching.

4. Mentor Graphics rewards individuals who take risks.

61. People at Mentor Graphics are rewarded for making the effort to see the viewpoint of others.

68. At Mentor Graphics, people who 'push back' are rewarded.

\begin{tabular}{|cccccc|}
\hline Alpha & Mean & Std. Dev. & Count & Min. & Max. \\
\hline .783 & 3.92 & 1.07 & 135 & 1.5 & 6.5 \\
\hline
\end{tabular}

Figure 40, Rewards variable.

The scores on these items are low (below 4.0, i.e., subjects "slightly disagree") indicating that some of the behaviors that are being suggested by the simulation are ones that the subjects believe are not rewarded in the organization. This is seen as an example of participants' non-simulation experience that would be expected to work to inhibit the attitude change observed in the dependent variables.

The questionnaire items in Figure 41 were included to provide some assessment of employees' perception of changes in the organization. These items address "vision", "partnerships" and other concepts related to the Quality Growth frame of reference. The simulation model suggests that without extensive effort, Mentor Graphics will gradually become more "bureaucratic". While these are only a crude measurement - they were made at one point in time and ask for judgements about change, rather than 
actually making measurements at different times - the responses are consistent with the statement that MGC was gradually becoming more bureaucratic during the two years prior to the May 1990 questionnaire. The response to item 15 is indicative of change. During the interviews in 1987 employees consistently commented that the company had a strong vision that was guiding it. In May of 1990, 135 employees "slightly agree".

The items in Figure 42 were included as background support for the discussion of "bureaucracy" and "small company atmosphere". The response to item 1 indicates the strong preference of employees for the latter. The Beta role incorporated the value of resisting bureaucracy to maintain the small company atmosphere and this role element was tentatively selected based on the researchers' perceptions during the interviews. Those perceptions are supported by responses to item 1 .

\begin{tabular}{|c|c|c|c|c|}
\hline Questionnaire Items & Mean & Std. Dev. & Min. & Max. \\
\hline $\begin{array}{l}\text { 15. At Mentor Graphics there is a long term vision of } \\
\text { where the company is going. }\end{array}$ & 5.35 & 1.61 & 1 & 7 \\
\hline $\begin{array}{l}\text { 24. There is a greater effort put into building } \\
\text { partnerships at Mentor Graphics today than there } \\
\text { was two years ago. }\end{array}$ & 3.79 & 1.36 & 1 & 7 \\
\hline $\begin{array}{l}\text { 27. The shared vision in the department where I work } \\
\text { is as strong or stronger now than it was two years } \\
\text { ago. }\end{array}$ & 3.96 & 1.89 & 1 & 7 \\
\hline $\begin{array}{l}\text { 67. During the last } 2 \text { years, Mentor Graphics has lost } \\
\text { some of its small company atmosphere. }\end{array}$ & 5.74 & 1.23 & 1 & 7 \\
\hline $\begin{array}{l}\text { 74. On average, the people in the group where I work } \\
\text { feel more in control of their own destiny today } \\
\text { than they did two years ago. }\end{array}$ & 3.50 & 1.71 & 1 & 7 \\
\hline
\end{tabular}

Figure 41. Organizational change questions. 


\begin{tabular}{|c|c|c|c|c|}
\hline Questionnaire Items & Mean & Std. Dev. & Min. & Max. \\
\hline $\begin{array}{l}\text { 1. I prefer working in an organization with a 'small } \\
\text { company atmosphere' to working in a bureaucratic } \\
\text { organization. }\end{array}$ & 6.39 & 1.02 & 1 & 7 \\
\hline $\begin{array}{l}\text { 77. Compared to other companies of similar age and } \\
\text { size, Mentor Graphics has a minimal amount of } \\
\text { bureaucracy. }\end{array}$ & 4.47 & 1.32 & 1 & 7 \\
\hline $\begin{array}{l}\text { 93. Today the biggest threat to the success of Mentor } \\
\text { Graphics is the problems that result from growth } \\
\text { within the company. }\end{array}$ & 4.50 & 1.86 & 1 & 7 \\
\hline $\begin{array}{l}\text { 8. Mentor Graphics is the best company I've ever } \\
\text { worked for. }\end{array}$ & 5.84 & 160 & 1 & 7 \\
\hline
\end{tabular}

Figure 42, Bureaucracy support questions.

The items in Figure 43 are typical of the filler items. These were taken from statements made by employees during the interviews but the content of these items did not find its way into the frames of reference and they were therefore not addressed by the simulation or considered targets for change. 


\begin{tabular}{|c|c|c|c|c|}
\hline Questionnaire Items & Mean & Std. Dev. & Min. & Max. \\
\hline $\begin{array}{l}\text { 5. For the most part, upper management at Mentor } \\
\text { Graphics does not use the "management by } \\
\text { walking around" philosophy. }\end{array}$ & 5.81 & 1.34 & 1 & 7 \\
\hline $\begin{array}{l}\text { 11. At Mentor Graphics the top management is } \\
\text { available and willing to listen to the ideas of any } \\
\text { of the employees. }\end{array}$ & 4.47 & 1.68 & 1 & 7 \\
\hline $\begin{array}{l}\text { 17. The actions of managers at Mentor Graphics are } \\
\text { frequently driven by short term pressures, such as } \\
\text { the next release or next quarter's financial } \\
\text { performance, to the detriment of longer term } \\
\text { corporate goals. }\end{array}$ & 5.85 & 1.38 & 2 & 7 \\
\hline $\begin{array}{l}\text { 23. Mentor Graphics under-invests in training for } \\
\text { employees. }\end{array}$ & 4.72 & 1.84 & 1 & 7 \\
\hline $\begin{array}{l}\text { 25. Most of the people at Mentor Graphics expect that } \\
\text { their job responsibilities will change completely } \\
\text { over the next } 2 \text { to } 3 \text { years. }\end{array}$ & 4.71 & 1.56 & 1 & 7 \\
\hline $\begin{array}{l}\text { 38. There are many times that form or appearance is } \\
\text { given a higher priority than function at Mentor } \\
\text { Graphics. }\end{array}$ & 4.85 & 1.65 & 1 & 7 \\
\hline $\begin{array}{l}\text { 40. We sometimes show little patience with customers } \\
\text { who are not as technically sophisticated as we are. }\end{array}$ & 4.02 & 1.42 & 1 & 7 \\
\hline $\begin{array}{l}\text { 43. It is very expensive to go to the market with a } \\
\text { new product and then have to do major rework to } \\
\text { get it right. }\end{array}$ & 6.54 & 0.98 & 1 & 7 \\
\hline $\begin{array}{l}\text { 46. At Mentor Graphics, decisions are usually pushed } \\
\text { down to the lowest appropriate level. }\end{array}$ & 3.10 & 1.85 & 1 & 7 \\
\hline $\begin{array}{l}\text { 49. People at Mentor Graphics work more closely as a } \\
\text { team than do people in most other high tech } \\
\text { companies. }\end{array}$ & 4.32 & 1.39 & 1 & 7 \\
\hline $\begin{array}{l}\text { 53. If the business environment were poor, Mentor } \\
\text { Graphics would do almost anything rather than lay } \\
\text { off people. }\end{array}$ & 4.96 & 1.69 & 1 & 7 \\
\hline
\end{tabular}

Figure 43, "Filler" questions. 


\section{CHAPTER VI}

\section{SUMMARY AND DISCUSSION}

\section{SUMMARY}

This first section of this chapter provides an 'executive summary' of the study findings. The following section is a more elaborate discussion of results, observations and speculations about what was learned in conducting the research. Figure 44 presents a causal model which is slightly different than the one presented in the statement of Research Questions (Chapter 3). The modifications reflect the inclusion of the "Role-Culture Contrast" variable which was not in the study proposal as well as the conclusions to the research questions which are indicated by arrow type. Figure 45 provides a summary of the conclusions for each research question.

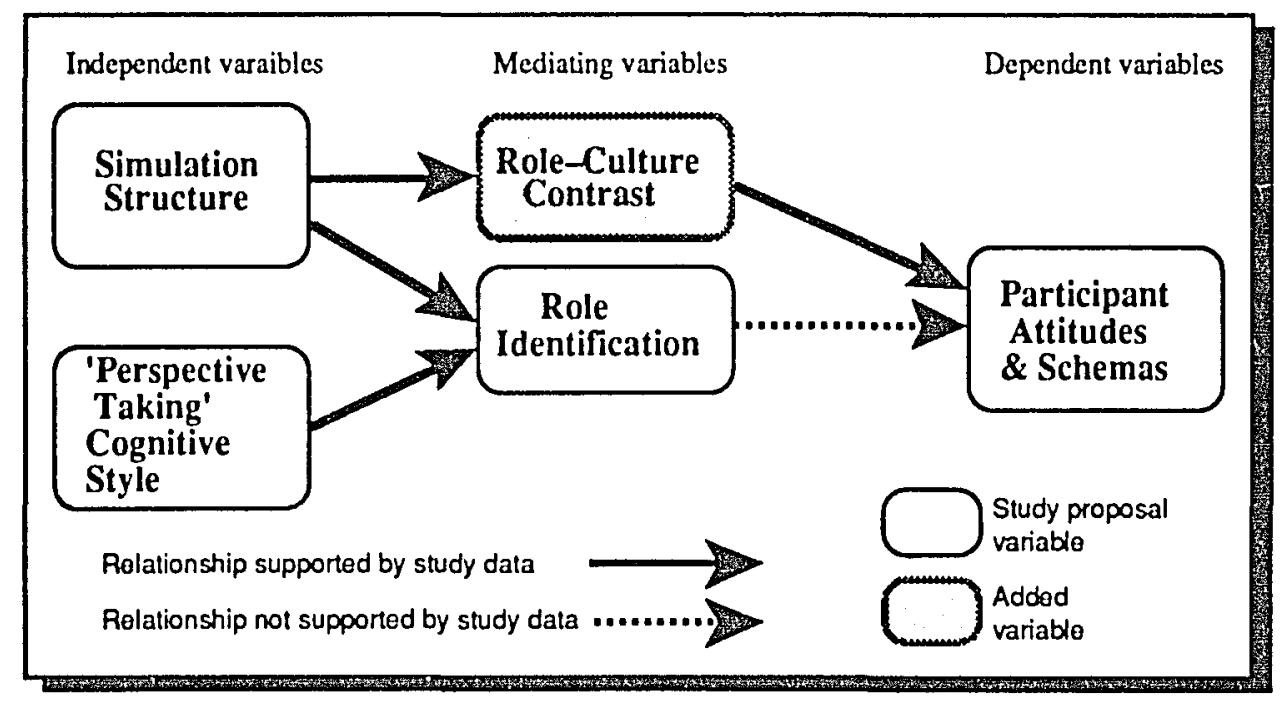

Figure 44, Modified causal model. 
Research Question 1 examined three demographic variables, and a 'simulation delivery' variable to determine if they were related to change in subject attitudes. There were no hypotheses associated with this research question because there were no a priori beliefs about whether these variables were related to attitude change (and it is therefore not represented in Figure 44). These variables were included as controls. The intent was that if any relationships were uncovered, subsequent analysis could either include these variables to correct for their influence or modify the interpretation of the results accordingly.

\begin{tabular}{|c|c|c|}
\hline Research Question & Conclusion & Table \\
\hline $\begin{array}{l}\text { 1. Are demographic variables related to } \\
\text { simulation-induced attitude change? }\end{array}$ & $\begin{array}{l}\text { Small effects, no major } \\
\text { biases detected in data. }\end{array}$ & $\begin{array}{l}\text { III-VIII } \\
\text { p } 118-123\end{array}$ \\
\hline $\begin{array}{l}\text { 2. Can simulation experience (specifically the } \\
\text { WADA simulation) cause attitude chan'se? }\end{array}$ & $\begin{array}{l}\text { Yes for all treatment groups, } \\
\text { no change for control group. }\end{array}$ & $\begin{array}{l}\text { IX-XII } \\
\text { p } 126-128\end{array}$ \\
\hline $\begin{array}{l}\text { 3. Does including certain structural attributes in } \\
\text { the simulation design increase 'Role } \\
\text { Identification' (and R-CC) for participants? }\end{array}$ & $\begin{array}{l}\text { Yes, significant differences for } \\
\text { Role Identification . Greater } \\
\text { difference observed for R-CC. }\end{array}$ & $\begin{array}{l}\text { XIII } \\
\text { p } 133\end{array}$ \\
\hline $\begin{array}{l}\text { 4. Is the cognitive style of the participant, as } \\
\text { measured by the cognitive subscales of the } \\
\text { Interpersonal Reactivity Index, a factor } \\
\text { predicting 'Role Identification' during a } \\
\text { simulation experience? }\end{array}$ & $\begin{array}{l}\text { Yes for the IRI Perspective } \\
\text { Taking' scale, no for the } \\
\text { 'Fantasy' scale. }\end{array}$ & $\begin{array}{l}\text { XV-XVI } \\
p 136-137\end{array}$ \\
\hline $\begin{array}{l}\text { 5. Is the level of subjects' Role Identification' } \\
\text { a factor that predicts attitude change caused by } \\
\text { simulation experience? }\end{array}$ & $\begin{array}{l}\text { No support for 'Role } \\
\text { Identification'. Significant } \\
\text { R-CC effect observed. }\end{array}$ & $\begin{array}{l}\text { XVII-XIX } \\
\text { p } 139-141\end{array}$ \\
\hline $\begin{array}{l}\text { 6. Does including certain structural attributes in } \\
\text { the simulation design enhance its ability to } \\
\text { produce attitude change beyond that governed by } \\
\text { the mediating variable? }\end{array}$ & $\begin{array}{l}\text { No, there was greater change } \\
\text { for the Simplified Simulation } \\
\text { group. }\end{array}$ & $\begin{array}{l}X X \\
\text { p } 142\end{array}$ \\
\hline $\begin{array}{l}\text { 7. Are there any "system-level" effects (enduring } \\
\text { frame of reference changes) accompanying } \\
\text { simulation-induced attitude change? }\end{array}$ & $\begin{array}{l}\text { There was some evidence for } \\
\text { a "system-level" effect. }\end{array}$ & $\begin{array}{l}\text { XXI-XXIV } \\
\text { p } 144-147\end{array}$ \\
\hline
\end{tabular}

Figure 45. Summary of Research Question conclusions. 
There were no significant differences in attitude change related to 'simulation delivery' although there were considerable differences between the facilitators. This suggests that the effect of the WADA simulation was stable, and data from the two sessions can be combined in further analysis.

Subject age and gender had no significant influence on the amount of change in the global attitude variable "Quality Growth". In each case there was significant change in two subscale variables related to these demographic variables. Length of employment was significantly correlated with change in the "Quality Growth" scale though the amount of variance it accounted for was small.

Overall, the demographic variables had little influence on attitude change. This was fortunate because the project did not achieve the original intent of a true field experiment. The delay between the pre-simulation questionnaire and the initial August session, and the need for and delay prior to the October session, reduced the quality of the data collected. This resulted in the demographic data not being incorporated in subsequent statistical analysis.

Research Question 2 compared pre and post-simulation attitude measurements within groups (Tables IX to XII). For the Control group, who had the greatest time period between questionnaires, there was essentially no change. This was encouraging because the five months between questionnaires for this group is considerably longer than the norm for Control group test-retest measurements.

Each of the three treatment groups did have significant change for the "Quality Growth" dependent variable. The Simplified Simulation appeared to have the greatest change when magnitude of change and number of subscales 
indicating significant change are considered. However, this group also had the greatest proportion of female subjects and the longest average length of employment, factors which correlated positively with greater attitude change. The conclusion that subjects in the treatment groups changed their attitudes, while those in the Control group did not, is well supported. This finding confirms the results of other simulation studies under more rigorous experimental conditions. The Methodology Chapter documents in more detail the simulation structures believed to account for this effectiveness.

Research Question 3 compares data across treatment groups to see if the simulation versions resulted in different levels for the mediating variables that operationalized 'Simulation Experience'. There was significantly greater Role Identification and perceived Role-Culture Contrast for subjects in the Enhanced Simulation than in the Simplified Simulation as predicted. Also consistent with predictions, there was no difference between subjects in the Enhanced Simulation and those in the Video Control group (Table XIV), indicating that viewing the video was not responsible for these differences.

These findings confirm the general hypothesis that different simulation structures can cause different simulation experiences for subjects. The more specific conclusion that the different simulation structures used in this study can induce differential levels of subject role identification is important for future research addressing that dimension of simulation experience.

Research Question 4 asks if the participants' cognitive style is a factor predicting identification with a simulation role. The Perspective Taking subscale of the IRI was a significant factor predicting identification with the Beta role as shown in Table XV. (It was also a significant predictor of subject reports of identification with the Alpha role). It was not related to the Role- 
Culture Contrast variable. The Fantasy subscale of the IRI was not a significant factor predicting either role identification or role-culture contrast.

This finding is perhaps the best documentation to date that differences between individual subjects can produce differences in their perceptions of the simulation experience, and more specifically, that they can perceive simulation roles differently. This is hardly a surprising finding but previous researchers have not recorded it with a quantitative measurement. Although the function of role identification in attitude change remains in doubt (see Research Question 5) it will likely remain a topic of interest in simulation research. The inclusion of some measure of subject cognitive style as a control in future simulation research on role identification now appears warranted.

Research Question 5 explored the final link in the causal model (Figure 44) by testing if either mediating variable relates to attitude change. The conservative t-Test in Table XVII indicated no significant difference in attitude change between those subjects reporting "high" and "low" role identification. However the study's conclusion is that this needs further research. The strength and nature of the findings in this study are insufficient to suggest that the role identification concept be abandoned.

The Role-Culture Contrast variable had a non-linear relationship to attitude change (Table XVIII). Subjects with very high and very low R-CC scores had little change while subjects while mid-range scores had significantly greater attitude change (Table XIX). The conclusions about Role-Culture Contrast are speculative and further research is required to verify these observations. Still, this finding indicates an important new topic 
for simulation research. A possible explanation for the lack of a significant relationship for Role Identification is that it was masked by R-CC.

Research Question 6 was written with the expectation that subjects in the Enhanced Simulation group would have greater attitude change than those in the Simplified Simulation. Table XX indicates that this did not occur. The relative effectiveness of the different simulation versions are considered in detail in the Discussion section.

Research Question 7 examined several indicators to see if there was any evidence that there was some change at a more global level that a single attitude. That more global level of analysis might be labeled "schema change" or "frame of reference change" or "role incorporation". Better measurements of subjects' cognitive maps would be required to assess proof of such change. The conclusion here is that there was some evidence to support the idea that subjects learned the Beta role and incorporated it into their organizational schemas.

\section{DISCUSSION OF RESULTS}

\section{Primary Simulation Design Goal - Attitude Change}

Tables IX through XII show that the WADA simulation was effective in producing attitude change, the primary design goal. These results were obtained under experimental conditions, outlined in Figure 46, more rigorous than those of most previous simulation studies addressing attitude change. While one view is that this finding only confirms previous work, the use of adults in a work setting addressing attitudes that senior management judged important to the success of the organization, supports a stronger claim for simulation effectiveness than that made in previous studies. In addition it 
suggests that those interested in directing organizational change may want to consider simulation as an intervention tool.

There was significant change in the global "Quality Growth" variable for each treatment group. Examination of changes in the subscale variables contributes to additional understanding of the simulation's effectiveness. Figure 18 (page 80 ) displays the hierarchical structure of the frame of reference.

There was also significant attitude change for the capstone variable in the Quality Growth frame of reference that was attributable to simulation experience (Table XXI). Change in this attitude about whether it is "possible to avoid bureaucracy" was away from a position that was commonly expressed during the interviews and toward greater empowerment of employees. The content addressed by this variable made it the most open to refutation from participants' non-simulation experience.

There was significant change concerning subjects' "Responsibility to Act", especially among females. There was little change for the "Opportunities to Act" dependent variable. Here males had significantly more change than females. These two variables at the mid-level of the Quality Growth hierarchy did not receive explicit emphasis in the simulation. Instead they were conclusions subjects were expected to draw from their simulation experience. Relatively small modifications could give them greater emphasis in future simulation use but an important question for the organization is whether aspects of the organizational reality hinder change.

The remaining mid-level variable, "Definition of Bureaucracy" was directly and repeatedly emphasized in the simulation classes. It is somewhat surprising that the change was so small and uneven. 
1. The questionnaire items were drawn from comments by members of the Mentor Graphics culture. This process increased the likelihood that subjects understood and related well to the constructs it contained.

2. Subjects names were randomly drawn from the payroll list and randomly assigned (in a blocked design) to treatment groups, thus addressing the concern of Butler et al. (1988) about assigning existing groups to treatments.

3. Questionnaire reliability was measured and found acceptable for all dependent variables. It is presented in Appendix D.

4. The simulation designer was not present during the classes. Invitation to classes, facilitation and debriefing was conducted by the client organization, thus eliminating the Pierfy's concern of developer bias.

5. Attitude measurement was separate from the simulation experience in time and context. Subjects contacted in post-study interviews had not made a conscious connection and none were aware that they had been subjects in an experiment. This removed Pierfy's concerns that the pre-test could influence the simulation experience, or that a demand effect influence the pest-test responses.

6. Post-simulation measurement indicated the presence of a strong effect three weeks after the treatment. It is often assumed that the impact of organizational interventions decays quickly. This duration of effect shows that the measured change was not a transient response.

7. Subjects were employees of an organization and regularly encountered the issues of the simulation and expressed attitudes that were targeted for change. Because of both subjects' involvement with the content, and their being adults, it is believed that they were more committed to their existing attitudes, and therefore more resistant to change, than were the students who were subjects in previously published research.

Figure 46. Summary of refinements in experimental procedures.

The largest change occurred in employee beliefs about whether certain employee actions can help maintain the "small company atmosphere" and reduce "bureaucracy" (dependent variable 5). This is a clear example of a change toward more empowerment for employees in this domain. A relatively large change was also measured for "Need for Change" (dependent variable 6). These two variables are at the lowest (most concrete) level of the Quality Growth frame of reference. This places them closest to actions the 
employees might take in their daily affairs. It was also true that the content they address is less refutable with their experience. Indeed, the "Need for Change" is consistent with a more general 'continuous improvement' theme which receives support within the organization.

The other variables at this bottom level were "Partnership" and "Coaching". These were residues of much larger frames of reference that were created while analyzing the ethnographic data and constructing the pilot questionnaire. Constraints encountered in building the simulation led to a dramatic de-emphasis of this content as well as the loss of the measurement of syllogistic structure among elements of these subscales. It was therefore not surprising that little change was recorded for these variables.

\section{Influence of Demographic Variables}

The finding that change in the "Opportunities to Act" and

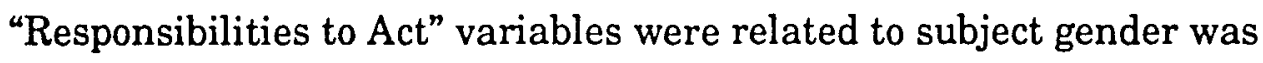
surprising. It probably indicates issues specific to this organization and is an example of how the organizational context can influence the effectiveness of interventions. There was not evidence in this study that would lead to a conclusion that females are more or less likely to be influenced by simulation experience.

Also of interest was the relationship of length of employment in the client organization to attitude change. Employees who had been with the firm longer were more likely to show greater attitude change (Table VIII). This result is best explained by noting that the organizational culture was observed to change during the " 8.0 " project (described in Appendix $\mathrm{C}$ ) and that subjects employed less than two years would not have had as much 
exposure to the "small company atmosphere" that was the core of the ideal Beta culture presented by the simulation.

\section{Secondary Design Geal - Differential Role Identification}

The second simulation design goal was producing differential role identification between the Simplified Simulation and Enhanced Simulation treatment groups. The analysis was shown in Table XIII. Questions may remain about the accuracy or reliability of the self report used to measure role identification, however this study goes beyond that of previous work where there was no attempt to quantify the extent of identification with a simulation role. The questions used to measure role identification in the simulation were similar to those in the "Perspective Taking" subscale of Davis' IRI, an instrument whose reliability has been independently demonstrated (Davis 1980). This similarity, together with the internal reliability of the "Role-ID" variable, supports the conclusion that different levels of role identification were created by the different simulation structures. The results of Tables XIII and XIV give some indication of how the questions used to measure identification could be modified to produce a better instrument.

The question measuring perceived contrast between the simulation role and the MGC culture (R-CC) demonstrated that the treatment conditions produced different perceptions of the Beta cultural role. During simulation design it was assumed that greater role identification and greater roleculture contrast would act together to induce greater attitude change. The finding of a non-linear relationship between role-culture contrast and attitude change is central to the conclusions of this study. 


\section{Role Identification and Attitude Change}

The hypothesized relationship of greater role identification producing greater attitude change was not affirmed with the t-Test comparison in Table XVII. This finding is in conflict with the suggestions of earlier work (Livingston and Kidder 1973; Williams 1987) and the "system-level" model of change hypothesized in this study.

Exploratory multiple regression analysis found a significant relationship for the Simplified Simulation group only when subject age and lengt's of employment were included in the models. Given that there are no competing causal models relating simulation to attitude change, and considering the explanation below of the impact of the role-culture contrast variable, a conclusion that role identification is not a factor that mediates attitude change would go beyond the strength of this study's findings. Instead the weak conclusion that "more research is needed" is what this study offers.

In conflict with a priori predictions, the Enhanced Simulation group which had greater role identification, had significantly less attitude change than the Simplified Simulation Group. The a priori expectation was that if role identification had no effect, then the same amount of change would be seen in these groups. The result instead suggests that some other factor influenced the subjects and, as the Simplified Simulation group received a subset of the enhanced treatment, it is the latter group that must have been influenced by this unknown factor. Perceived Role-Culture Contrast, a second mediating variable, is one factor that can explain the differences in attitude change between treatment groups. 


\section{Role-Culture Contrast and Attitude Change}

Treatment group (simulation structure) was found to be a significant factor predicting Role-Culture Contrast (Table XIII), with the Enhanced Simulation group reporting significantly greater Role-Culture Contrast. Perceived contrast between the Beta simulation role and the culture of the client organization was a significant factor predicting attitude change (Tables XVIII and XIX), though this relationship was non-linear. That model of the relationship between Role-Culture Contrast and attitude change is theoretically plausible as discussed in the Results chapter under Research Question 5. Thus it appears that excessive Role-Culture Contrast was created for some subjects receiving the Enhanced Simulation and Video Control treatments and that this limited the amount of attitude change for those subjects. One possible conclusion would be that the strong RoleCulture Contrast effect for these subjects eclipsed the effect of Role Identification.

This possible conclusion is supported by observations that were made prior to data analysis. In discussions with the facilitators after the classes they quickly pointed out that the majority of subjects in the Enhanced Simulation and Video Control groups "threw everything into culture development" while playing the Beta culture in their second game and did not focus on competing for task points with the other teams. That is, these subjects adopted a goal of maximizing their quality scores, a course of action that precluded maximizing their task scores. This behavior represents a gross misunderstanding of the definition of winning in the Beta cultural role. The Betan goal is to maximize task scores while maintaining the minimum quality score necessary to avoid "bureaucracy". The required minimum does 
represent much greater investment in culture development than in the Alpha game, but not "everything". Whether this outcome represented a lack of understanding on the part of the facilitators about the Beta culture, or about how to deliver the simulation, or perhaps poor calibration of the simulation variables is not known. Post study use of a modified WADA simulation in the client organization saw participants correctly pursue the Beta goal of maximizing task points while avoiding bureaucracy.

A probable result was that the credibility of Beta role as a model of the real world was downgraded for subjects in the Enhanced Simulation and Video Control groups. In experiencing the Beta culture they no longer competed against other teams to earn "task points" which they were told represented "external markers of organizational success" including earnings, market share, stock price, and product innovation. They likely perceived it as a flawed model, one that could not be applied to their organizational reality. This is consistent with several facilitator comments during the debriefings, and under such conditions it would be expected that experiencing the Beta role would have a reduced impact on attitude change.

Subjects in the Simplified Simulation, playing the Beta role in their first and only game, did not adopt this unexpected goal. Instead, not having experienced "bureaucracy" in an Alpha game, they were reluctant to commit resources to building the culture (consistent with the existing attitudes of the MGC culture) and were as concerned with competing for the most task points as were subjects in the other treatment groups during their first (Alpha) play of the simulation. The facilitators commented that they had to put considerable effort into pressing Simplified Simulation subjects to maintain 
the small company atmosphere. (This effort became one uncontrolled factor that distinguished the facilitation of the treatment groups.)

The Constructivist perspective suggested that the credibility of the simulation role, as well as identification with that role, would be factors that infiluence whether subjects ultimately integrate the role into their cognitive schemas (Figure 12). A loss of credibility for the Beta role in the Enhanced Simulation and Video Control groups would explain the results observed. It could mask the effect of differences in Role Identification because identifying with a role that is not credible would not be expected to result in subjects incorporating that role into their organizational schemas. Less attitude change would be predicted in this situation. Because it was believed a priori that subjects in each treatment condition would ascribe the same credibility to the Beta role, there was no attempt to assess the credibility of the role for subjects. Obviously this is a consideration for future research.

\section{Other Plausible Explanations for Observed Results}

A number of plausible explanations exist for the unexpected results of greater role identification and coincidental less attitude change in the Enhanced Simulation. One is a possible interaction with the content of the attitudes targeted for change. It would be consistent for subjects who favor an external locus of control approach to be predisposed to identify with simulation roles. These could be people who more often look to others for role models and therefore would report high levels of identification. The Beta cultural role suggests a change to an internal locus of control, something which these subjects may find less appealing. This would result in lessened attitude change (toward becoming internal) for those who tended toward greater role identification. Said another way, the high identifiers could have 
had greater preference for the Alpha role. Because no locus of control assessment was made for the subjects this remains a speculative but plausible explanation.

A second explanation is that the two-role simulation structure may have suggested a bi-stable schema (including Alpha and Beta roles) rather than a single ideal schema as intended. This could have influenced the incorporation of the simulation experience into subjects' organizational realities and lessened attitude change.

Other explanations are possible. Although there was significant differences in role identification between treatment groups, this is the first simulation study to quantify those differences. It is unknown whether the identification levels achieved were "high" or "low" in comparison to that produced with other gaming-simulations. It is possible that the levels of identification observed in this study were uniformly so high that all subjects experienced its maximum effect and therefore the test for differences in indentification-related attitude change was dominated entirely by other factors. It is also possible, though considered far less likely, that the levels of identification measured in this study were so low that they failed to create any effect though one would have been observed if higher levels of role identification were obtained.

It is possible that some other structural feature of the simulation moderated attitude change for the Enhanced Simulation and Video Control groups. McGuire(1985:246) notes "Attitudes on target issues become more resistant to persuasion if, before they are attacked, their connections to attitudes on related issues are made more salient, especially when the attacking communication argues in an inconsistency-increasing direction." 
This suggests the possibility that something in the content of the Alpha role could have caused an anchoring effect, reducing the change induced by playing the Beta role. If McGuire's observation were to hold true in simulation settings, then a primary structural attribute of the enhanced version of WADA - the sequential comparison of contrasting roles in the order suggested by Bennett's empathy model - is self defeating.

The system-level model of change hypothesized in this study offers a contrasting position to McGuire's observation - that by shifting to a new frame of reference in an atmosphere of play, individual attitudes are never "attacked", systemic resilience is side-stepped rather than assailed. Playing the WINNING AT DESIGN AUTOMATION simulation with the order of roles reversed, (Beta game then Alpha game) could allow a test of the relevance of McGuire's observation.

Other explanations for the puzzling results of higher role identification and lower attitude change in the Enhanced Simulation group exist that do not repudiate the hypothesized causal model. For example, players in the Enhanced Simulation and Video Control groups may have felt "they had been had" after they played the Beta role and realized that the Alpha role was a suboptimal strategy. A feeling of being tricked could cause them to denigrate the usefulness of the experience and therefore produce less change. While no evidence suggests that this response occurred, it is one that subjects might not share publicly. Yet another explanation is that the longer classes led to fatigue for the Enhanced Simulation and Video Control groups, thereby blurring the simulations' effectiveness. (Simplified Simulation classes lasted about six hours while the other treatment classes averaged nearly eight.) 
Again there was no evidence that subjects were fatigued but class length and discussion time were uncontrolled variables.

Another possible explanation is that any role identification effect may be related to attitude content. The strongest link between role identification and attitude change observed in the exploratory regression analysis was for the "Need to Change" variable. (This was a multiple regression with Role ID, employment length and age as independent variables, $R^{2}=.60, F$ for Role ID $=31.8, \mathrm{~N}=37$.) Models for other subscales were far less significant. Those questionnaire items (Figure 24) measured whether subjects had transferred lessons learned in the simulation about the fictitious Beta culture to the client organization were they worked. While many factors might influence which subjects learn what lessons during a simulation, perhaps only subjects with greater role identification make the transfer of that learning to the nonsimulation domains where attitude measurement was made in this study. Measurement of the significance of role identification would then need to carefully discriminate what content was learned in a simulation (perhaps unrelated to role identification) and what content was transferred to knowledge domains outside the simulation. The context of attitude measurement would be very important, consistent with the comments by Palmerino et al. (1984).

A final explanation offered is that the facilitation of the simulation may have introduced a systematic bias into the results. Specifically, the delivery of the Enhanced Simulation may have been inadequate. The Enhanced Simulation was more complex and therefore more difficult to deliver. An adequate debriefing in particular requires special care as the facilitator must address the two cultural roles, the different awareness each has of the 
simulation model as well as some resolution of their juxtaposition. Review of the taped class discussions showed that the facilitators occasionally described the causal relationships in the simulation model incorrectly. It was observed in the discussion of 'Simulation Delivery' (Methodology section) that there was a steep learning curve for the facilitators during their week of classes and that they did not correctly incorporate all of the simulation features. There is not a great deal of evidence to support this explanation but it cannot be ruled out. Said another way, the only indicator for quality of delivery was the attitude change data. If one version did not have the change predicted, it cannot be determined if the hypothesized model or the delivery of the simulation was flawed.

\section{Qualitative Assessment of Simulation Effectiveness}

Magnitude of attitude change was the basis selected for assessing the effectiveness of the WADA simulation in this study. The greater change recorded for the Simplified Simulation, together with its shorter class time, could imply that this version is 'superior'. The vice president and trainer who facilitated the classes reached a different conclusion. Their comment was that those subjects in the Simplified Simulation "really got the short end of the stick". They strongly believed that the Enhanced Simulation provided a superior training experience. Even after learning of greater change in the Simplified Simulation group, they recommended that Mentor Graphics rent the Enhanced Simulation during 1992. They believed the experience of contrasting roles in the Enhanced Simulation was a superior training experience.

Magnitude of change is not the only possible evaluation criteria. Duration of effect, not measured in this study, is another important 
consideration. It is possible that the contrast of cultural roles in the Enhanced Simulation led to a greater duration of effect.

Participant enjoyment is another possible criteria for assessment of a training simulation. Lee and O'Leary (1971:344) concluded "Our findings clearly imply that student enioyment is absolutely necessary to achieve the more profound kinds of learning objectives addressed by simulation games." Making WADA an enjoyable experience was a goal throughout the design process. One of the questions asked by the software at the end of the simulation was, "I found the experience of playing the Beta culture in WADA

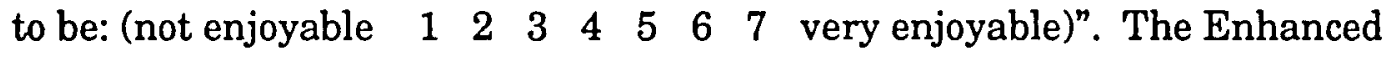
Simulation was rated slightly higher (average $6.41, \mathrm{~N}=41$ ) than the Simplified Simulation (6.15, $N=44$ ) when comparing data from all class participants. Nearly identical results were recorded for the subset of participants who completed questionnaires. The Video Control group fell between the other treatment groups $(6.32, \mathrm{~N}=37)$.

This measure of participant enjoyment had a positive relationship to reported identification with the simulation role. Greater identification was coincident with greater enjoyment. A simple regression of "Role $\mathrm{ID}^{\text {" and the }}$ "Enjoyment" question produced an $\mathrm{R}^{2}$ of $.12(\mathrm{~F}=11.5, \mathrm{p}=.0011, \mathrm{~N}=86)$. Enjoyment was also positively related to perceived role-culture contrast though the strength of this relationship and the amount of variance it explained was small $\left(\mathrm{R}^{2} .05, \mathrm{~F}=4.2, \mathrm{p}=.042\right)$. This relationship does support a conclusion that the reduced attitude change associated with large role-contrast was not caused by of a dislike of the Beta role.

Subjects were asked to evaluate the simulation in another question, "I think that playing WADA is a worthwhile experience for learning about the 
culture in the company where I work (not worthwhile $\begin{array}{lllllll}1 & 2 & 3 & 4 & 5 & 6 & 7\end{array}$ very worthwhile)". Again the Enhanced Simulation group gave the highest rating (6.29) though the Simplified Simulation was almost imperceptibly lower (6.25). The Video Control group average was 6.19.

At the end of each class the facilitator asked participants to rank the culture of Mentor Graphics on a dimension bounded by the Alpha and Beta cultures. (In the case of the Simplified Simulation, the MGC culture and the Beta culture role were used.) When asked if there had been any changes in the last two or three years there was nearly unanimous agreement that Mentor Graphics' culture had moved closer to Alpha. When asked "where would you like the Mentor Graphics culture to be" all indicated that they wanted the culture to be closer to that of Beta.

\section{Duration of Simulation Effect}

The question of duration of effect is often raised. The extensive change reported here was measured about three weeks after the simulation classes. It was therefore more than a transient response measured while the class experience was still fresh in the minds of the participants - a challenge that much of the previous simulation research cannot dismiss. This duration of effect allows substantial opportunity for the organization to reward and reinforce the change that the simulation introduced. The Constructivist perspective of social reality as a continual re-creation process suggests that in the longer term it will be organizational support for the changed attitudes, and not "duration of simulation effect", that determines the survival of those changes. The duration was demonstrated to be "long enough" and strong enough to justify using simulations to introduce attitude change, and new schemas, in organizational settings. If other facets of the organizational 
reality provide continued support those changes, if the new simulationsuggested reality is continually recreated, then it will endure. Clever simulation designers would create novel frames of reference that find support in existing aspects of the culture that are not targeted for change. Managers who want the simulation induced changes to endure should establish support mechanisms.

\section{Cognitive Style}

The "Perspective Taking" subscale of the IRI, one of two cognitive subscales in the Davis empathy measure, was a significant factor, independent of simulation structure, that predicted subject reports of role identification (Table XV). This finding is neither surprising nor enlightening given the similarity between the role identification questions (Figure 36) and the Perspective Taking scale of the IRI (questions 86 - 92 of the questionnaire, Appendix D). It says only that subjects who indicate on a questionnaire that they adopt the perspectives of others will also report they adopted a role they were asked to play in a simulation. It is reassuring to know that subjects respond consistently to questions, and this conveys some added support for the reliability of these instruments. But whether they did in fact "identify" with that role more than those who answered differently, and what exactly "identify" means remains elusive.

Given the condition that those who design or use simulations cannot control the cognitive style in the populations they address means that this knowledge may be of little pragmatic value. Further, the results of this study leave uncertain the effect of role identification. It is likely that other mediating variables can dominate the role identification effect thereby reducing its importance. 
Interestingly, the "Perspective Taking" scores for treatment group subjects in this study were confined to the upper half of the seven point range (Figure 47). In populations with a broader range of responses to this instrument, it is possible that a greater range of influence on role identification would be measured.

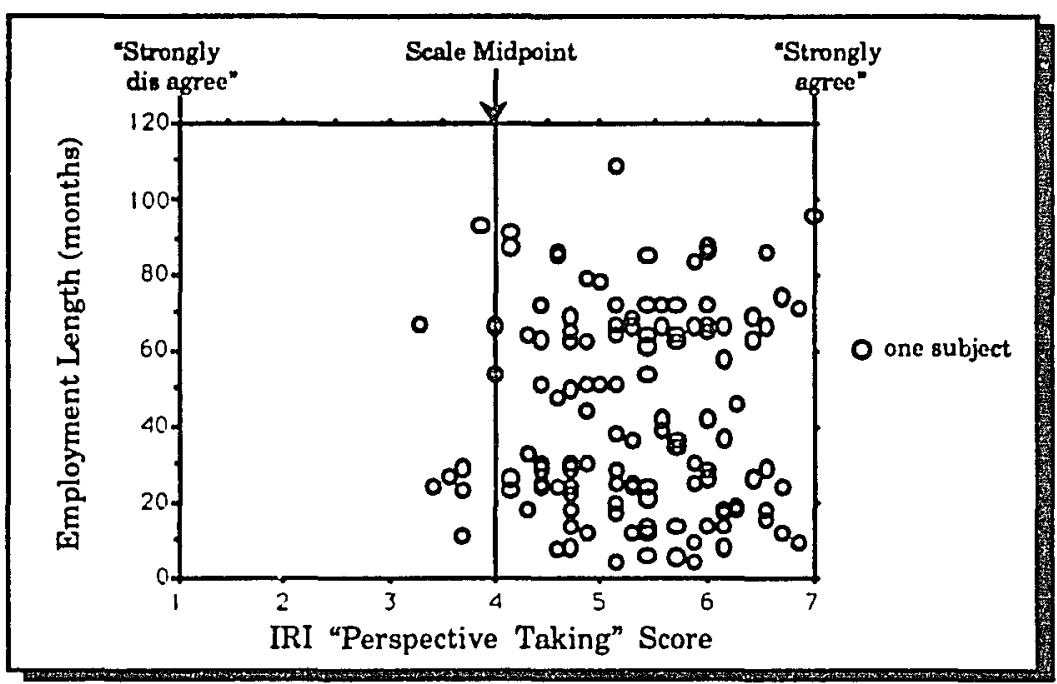

Figure 47. Range of subject's Perspective Taking scores.

The finding of a relationship between cognitive style and role identification is consistent with the hypothesized causal model involving the linkage of simulation structure and role identification to attitude change. The selection of this instrument and prediction of a significant effect were derived from that theoretical model.

\section{CONCLUSIONS}

Substantial attitude change was demonstrated with a simulation designed to support role identification and to suggest contrast between a simulation role and the organizational schemas observed during Phase 1 of 
the project. The attitudes that changed were well integrated in the participants' views of organizational reality. This study therefore provides considerable evidence about the effectiveness of simulations to produce change.

Several measures of change indicated that the frame of reference suggested in the simulation role was incorporated into participants' views of organizational reality. This suggests that in addition to attitude change, a more global change in participants' cognitive models - change in a system of attitudes which was here termed a "frame of reference" - may be feasible with simulation interventions.

The evidence gathered in this study supports the modified causal model shown in Figure 44. Simulation structure was found to significantly influence both Role Identification, and perception of Role-Culture Contrast. The design of the simulation structure which produced these results was guided by a communication theory. The elements of the design which are believed to have created the role identification and role-culture contrast have been described.

Greater role identification did not correspond to significantly greater attitude change, contrary to study hypotheses. High levels of Role-Culture Contrast may have overshadowed the effect of role identification and other uncertainties remain. This study leaves unanswered the question of whether role identification plays a role in simulation-induced attitude change.

The effect of Role-Culture Contrast, in particular its non-linear relationship to attitude change, is of considerable importance to simulation designers. While this effect is consistent with predictions implied by Constructivism, during simulation design it was believed that the contrast 
offered by the simulation role could not be so great as to cause reduced attitude change. None of the simulation research in the literature review mentioned the concept of contrast magnitude or the possibility of it being excessive, although the subject of dissonance is arguably the largest single topic in the attitude change literature. Perhaps simulation designers have naively assumed that in an atmosphere of play and fictionalized roles participants would discount any mis-scaling of role dissonance. Or perhaps as Palmerino et al. (1984) observe about attitude change researchers, they have simply failed to study broadly enough the context of their interventions and thus have not observed the phenomena.

"Role-Culture Contrast" was given this label because it represented the perceived difference between the simulation role and the culture of the client organization. "Role-Schema Contrast" is considered to be a more generic term, applicable for other research where the topic of the simulation is likely to be something other than the culture of a specific organization. In that case, the contrast that would be important would be that between the simulation role and the participant schemas activated by the simulation experience. Previous simulation research has not undertaken the extended effort in Phase 1 of this study to ascertain the mental maps that participants have about the domains addressed by the simulation. The effect attributed to "Role-Schema Contrast" in this study strongly suggests that future research on simulation effectiveness should measure and control for subject assessments of role credibility, role relevance and perceived social support for roles as suggested by Figure 12 .

The seven-item "Perspective Taking” subscale of Davis' Interpersonal Reactivity Index was a significant predictor of identification with the 
simulation role. This finding indicates that some individuals are predisposed to more strongly identify with the roles in a simulation and therefore may be more likely to change their attitudes as a result of simulation experience. A related conclusion that could be drawn from this finding is that those choosing among intervention options could temper their expectations for simulation-induced change according to the perspective taking style of the participants.

Constructivism was a useful theory for guiding simulation development. The use of ethnographic methods to collect qualitative data as a base from which the simulation was constructed is judged to be a very worthwhile effort. Bennett's model of empathy, while not subject to a quantitative test of effectiveness in this study, was found to be useful in suggesting a number of simulation features. Better explanation of result variability would have been likely had all of the mediating variables suggested by Constructivism been measured (Figure 12).

Several structural attributes of simulation design were incorporated in the WINNING AT DESIGN AUTOMATION simulation created in this study (Figure 8). The primary simulation structure of role and strategy blend in a multi-game simulation was considered to be very effective. HyperCard was found to be an excellent simulation development and delivery tool.

The evidence reported here indicates that simulations can be effective instruments to introduce attitude change in organizations. Attention to the socially constructed organizational realities - including incorporation of culturally relevant language and concepts into the simulation and roles - as well as careful design of the simulation experience are considered the keys to successful system-level interventions. 


\section{SUGGESTIONS FOR FUTURE RESEARCH}

- The relationship of identification with simulation roles to attitude change remains unclear. A few previous studies have suggested a causal relationship. This study was the first to quantitatively measure role identification and test for a relationship with attitude change. The analysis did not uncover such a relationship, but the findings were not strong enough to eliminate that possibility. Additional work with different simulations and better identification assessment instruments is needed to clarify the variables and relationships involved.

- The effect reported for "Role-Schema Contrast" strongly suggests there is a need in future simulation research to record participant assessment of the simulation roles. One useful contribution would be some generic role assessment instrument that could be used in many simulation settings. This could allow comparisons across studies and simulations. The social psychology literature should be reviewed to determine if such an instrument exists.

- Future research should control for inter-subject variability. Given the small cost of administering a seven item scale, the Perspective Taking subscale of the IRI is a good starting point for future research examining the relationship between simulation structure and role identification.

- One line of future research suggested by these findings is the possibility for some pre-simulation perspective-taking exercise to enhance simulation effectiveness. "Pre-briefing" as well as "debriefing" simulation experiences may create greater impact. Such an exercise can be seen as an extension of the instructions to identify with the simulation role used in the 
Enhanced Simulation in this study and by Williams (1987). Some of the work on creativity may be a useful starting point (Adams 1974).

- Training facilitators in the use of a particular simulation is important. Simulations such as WADA must be viewed as a complex technology requiring considerable prerequisite preparation. Playing the simulation two or three times is not sufficient to be a qualified facilitator, though that is what was accepted in this study. Given the limited preparation that many facilitators will make, future researchers should make secondary the concerns about experimenter bias. Especially in working with new simulations, they should facilitate simulation sessions themselves when unprepared or poorly trained facilitators are the option. Once some effectiveness has been demonstrated for a simulation, the experimenter bias issue can be addressed in additional work.

- The simulation structures used to support role identification are a possible basis for a taxonomy of simulations. There is a need for such a taxonomy to direct research into the relative effectiveness of simulation structures. 


\section{REFERENCES CONSULTED}

Adams, James 1974. Conceptual Blockbusting: A Guide to Better Ideas, W. H. Freeman and Company.

Aldrich, Howard, and Mueller, Susan 1982. "The Evolution of Organizational Forms: Technology, Coordination and Control", Research in Organizational Behavior, 4:33-87.

BAFA BAFA. Simile II, 1977. 218 Twelfth Street, P.O. Box 910, Del Mar, CA 92014.

Barley, Stephen R., Meyer, Gordon, and Gash, Debra, 1988. "Cultures of Culture: Academics, Practitioners and the Pragmatics of Normative Control", Administrative Science Quarterly, 33:24-60.

Barling, Julian, Kelloway, Kevin and Bremermann, Eric 1991. "Preemployment Predictors of Union Attitudes: The Role of Family Socialization and Work Beliefs", The Journal of Applied Psychology, 76:725-731.

Bem, Daryl J. 1970. Beliefs, Attitudes and Human Affairs, Wadsworth Publishing Company.

Bennett, Milton 1986. “Towards Ethnorelativism: A Developmental Model of Intercultural Sensitivity", in Cross Cultural Orientation: New Copnceptualizations and Applications, Paige, Michael (ed.), University Press of America, 27-69.

Bennett, Milton 1979. "Overcoming the Golden Rule: Sympathy and Empathy", Communication Yearbook 3, Nimo, D.(ed).

Bennett, Milton 1977. "The Forming / Feeling Model”, Doctoral dissertation, University of Minnesota.

Berger, Peter and Luckman, Thomas 1966. The Social Construction of Reality, Doubleday \& Company Inc.

Block, Peter 1987. The Empowered Manager, Jossey-Bass Inc.

Blumer, Herbert 1969. Symbolic Interactionism: Perspective and Method, Englewood Cliffs, NJ: Prentice Hall. 
Bowditch, James L. and Buono, Anthony F. 1990. A Primer on Organizational Behavior, second edition, John Wiley \& Sons.

Bowen, Donald D. 1987. "Developing a Personal Theory of Experiential Learning: A Dispatch from the Trenches" ${ }^{n}$, Simulation and Games: An International Journal of Theory, Design and Research, 18:192-206.

Bredemeier, Mary E., Bernstein, George and Oxman, Wendy 1982. "BAFA BAFA and Dogmatism / Ethnocentrism: A study of Attitude Change Through Simulation-Gaming", Simulation and Games: An International Journal of Theory, Design and Research, 13:413-436.

Bredemeier, Mary E. and Greenblat, Cathy Stein 1981. "The Educational Effectiveness of Simulation Games: A Synthesis of Findings" , Simulation and Games: An International Journal of Theory, Design and Research, 12:307-332.

Bredemeier, Mary E. 1978. "Providing Referents for Sociological Concepts", Teaching Sociology, 5:409-422.

Bruner, Jerome, Jolly, Allison and Sylva, Kathy, 1976. Play - Its Role in Development and Evolution, Basic Books Inc.

Burrows, Peter 1992. "Mentor Graphics recovers from its mighty fall", Electronic Business, June 84-90.

Butler, Richard, Markulis, Peter and Strang, Daniel 1988. "Where are We? An Analysis of the Methods and Focus of the Research on Simulation Gaming", Simulation and Games: An International Journal of Theory, Design and Research, 19:3-26

Calder, Bobby and Schurr, Paul 1981. "Attitudinal Processes in Organizations", Research in Organizational Behavior, 3:283-302.

Carmines, Edward G. and Zeller, Richard A. 1979. "Reliability and Validity Assessment", Quantitative Applications in the Social Sciences, Sage Publications.

Cherryholmes, C. H. 1966. "Some Current Research on the Effectiveness of Educational Simulations: Implications for Alternative Strategies", American Behavioral Scientist, 10:4-7.

Chaiken, Shelly and Stangor, Charles 1987. "Attitudes and Attitude Change", Annual Review of Psychology, 38:575-630.

Clemens, Walter C. 1976. "Games Sovietologists Play", Teaching Political Science, 3:140-159. 
Cooper, Joel and Croyle, Robert, 1984. "Attitudes and Attitude Change", Annual Review of Psychology, 35:395-426.

Corbeil, Pierre, 1988. "Teaching History Though Simulation Games: A Piagetian Perspectiven, paper presented at the annual North American Simulation and Gaming Association (NASAGA) confernce, Ashville, NC.

Crocker, Jennifer, Fiske, Susan and Taylor, Shelley 1984. "Schematic Bases of Belief Change", Chapter 10 Attitudinal Judgement, Eiser, J. Richard (ed), Springer-Verlag.

Cruickshank, Donald R. and Mager, Gerald M. 1976. "Toward Theory Building in the Field of Instructional Games and Simulations", Programed Learning and Education Technology, 13: 5-9.

Davis, Mark H. 1983. "Measuring Individual Differences in Empathy: Evidence for a Multidimensional Approach", Journal of Personalilty and Social Psychology, Vol. 44 No. 1 113-126.

Davis, Mark H. 1980. "A Multidimensional Approach to Individual Differences in Empathy", abstracted in the JSAS Catalogue of Selected Documents in Psychology, 10 (4).85.

Dawes Robyn M. and Smith, Tom L. 1985. "Attitude and Opinion Measurement" (Chapter 10) Lindzey, G., and Aronson, E. (eds) Handbook of Social Psychology, New York: Random House, 3rd edition.

Deal, Terrence E. and Kennedy, Allen 1982. Corporate Cultures, AddisonWesley Publishing Company, Inc.

Duke, Richard D. 1982. "Format for the Game--Logic or Intuition?", Simulation and Games: An International Journal of Theory, Design and Research, 11:27-34.

Duke, Richard D. 1981. "A Paradigm for Game Design", Chapter 7 in Greenblat, Cathy Stein and Duke, Richard D. (eds) Principles and Practices of Gaming-Simulation, Sage Publications.

Duke, Richard D. 1974. "Toward a General Theory of Gaming", Simulation and Games: An International Journal of Theory, Design and Research, 5:131-146.

Edwards, Kari 1990. "The Interplay of Affect and Cognition in Attitude Formation and Change", Journal of Personality and Social Psychology, 59:202-216. 
Edwards, K. L. 1971. "Student Evaluations of a Business Game as a Learning Experience”, Bethesda, MD: ERIC Document Reproduction Service 058142 .

Faria, A. J. 1987. "A Survey of the Use of Business Games in Academia and Business", Simulation and Games: An International Journal of Theory, Design and Research, 18:207-224.

Fine, Gary Alan 1983. Shared Fantasy: Role Playing Games as Social Worlds, University of Chicago Press.

Fletcher, Jerry L. 1971. "Evaluation of Learning in Two Social Studies Simulation Games", Simulation and Games: An International Journal of Theory, Design and Research, 2:259-286.

Fowler, Floyd J. Jr. 1984. "Survey Research Methods", Applied Social Reasearch Methods Series, Volume 1, Sage Publications.

Goffman, Erving 1959. Presentation of Self in Everyday Life, Doubleday Anchor Books.

Goodman, Fred 1988. Session on simulation design, annual North American Simulation and Gaming Association (NASAGA) conference, Ashville, N.C.

Gray, Virgina and Walcott, Charles 1977. "Simulation, Learning and Student Attitudes", Teaching Political Science, 4:295-305.

Greenblat, Cathy Stein 1988. Designing Games and Simulations, Sage Publications.

Greenblat, Cathy Stein and Duke, Richard D. 1981. Principles and Practices of Gaming-Simulation, Sage Publications.

Greenblat, Cathy Stein 1981. 'Further Exploxations on the 'Multiple Reality' Game", (Chapter 10) Greenblat, Cathy Stein and Duke, Richard D. Principles and Practices of Gaming-Simulation, Sage Publications.

Greenblat, Cathy Stein 1980. "Group Dynamics and Game Design", Simulation and Games: An International Journal of Theory, Design and Research, 11:35-57.

Hall, Eleanor G. 1979. "Simulation Gaming - A Device for Altering Attitudes about Sex Roles", The Gifted Child Quarterly, 23:356-360.

Hamid, P.N., and Flay, B.R. 1974. "Changes in locus of control as a function of value modification", British Journal of Social and Clinical Psychology, 13:143-150. 
Hickey, Joseph V., Thompson, William E., and Foster, Donald L. 1988. "Becoming the Easter Bunny, Socialization into a Fantasy Role", Journal of Contemporary Ethnography, 17:67-95.

Hofstadter, Douglas 1979. Gödel, Escher, Bach,: An Eiernal Golden Braid, Basic Books.

Jackson, M. W. 1979. "An Antipodean Evaluation of Simulation in Teaching", Simulation and Games: An International Journal of Theory, Design and Research, 10:99-138.

Jacobs, Ronald and Baum, Maryanne 1987. "Simulation and Games in Training and Development" ${ }^{n}$, Simulation and Games: An International Journal of Theory, Design and Research, 18:385-394.

Kahneman, Daniel, Slovic, Paul and Tversky, Amos 1982. Judgement under Uncertainty: Heuristics and Biases, Cambridge University Press.

Kaplan, Robert, Lombardo, Michael and Mazique, Mignon 1985. "A Mirror for Managers: Using Simulation to Develop Management Teams", The Journal of Applied Behavioral Science, 21:241-253.

Kawaski, Guy 1990. The Macintosh Way, Scott Foresman and Company.

Kelly, George 1955. A Theory of Personality: the Psychology of Personal Constructs, W. W. Norton and Co.

Kenny, Vincent (ed) 1988. "Radical Constructivism, Autopoiesis \& Psychotherapy", The Irish Journal of Psychology, Special Issue, Vol. 9, No. 1.

Kidder, Tracy 1981. The Soul of a New Machine, Avon Books.

Kroeber, Alfred L., and Kluckholm Clyde, 1952. Culture: A Critical Review of Concepts and Definitions, Cambridge: The Museum.

Kuhn, Thomas 1970. The Structure of Scientific Revolutions, Second Edition, The University of Chicago Press.

Langeler, Gerard H. 1992. "The Vision Trap", Harvard Business Review, 2:46-55.

Lee, Robert S., and O'Leary, Arlene 1971. "Attitude and Personality Effects of a Three-day Simulation", Simulation and Games: An International Journal of Theory, Design and Research, 2:309-347.

Leftcourt, Herbert M. 1982. Locus of Control: Current Trends in Theory and Research, Lawrence Erlbaum Associates. 
Lestor, J. P. and Stoil, M. J. 1979. "Evaluating a Role-specific Simulation", Simulation and Games: An International Journal of Theory, Design and Research, 10:167-188.

Linder, Darwyn, Cooper, Joel, and Jones, Edward 1967. "Decision Freedom as a Determinate of the Role of Incentive Magnitude in Attitude Change", Journal of Personality and Social Psychology, 6:245-254.

Linstone, Harold A. 1984. Multiple Perspectives for Decision Making, Elsevier Science Publishing Co.

Livingston, Samuel, and Kidder, Steven 1973. "Role Identification and Game Structure: Effects on Political Attitudes", Simulation and Games: An International Journal of Theory, Design and Research, 4:131-144.

Long, Edgar C. and Andrews, David W. 1990. "Perspective Taking as a Predictor of Marital Adjustment", Journal of Personality and Social Psychology, 59:126-131.

Louis, Meryl Reis 1985. "An Investigator's Guide to Workplace Culture", Organizational Culture, Frost, Peter et al., (eds), Sage Publications Inc.

Mandelbaum, David (ed.) 1949. Selected Writings of Edgar Sapir, xx.

Maturana, Humberto R. 1988. "Reality: The Search for Objectivity or the Quest for a Compelling Argument", The Irish Journal of Psychology, 9:25-82.

McGuire, William J. 1985. "Attitude and Attitude Change" (Chapter 19) Lindzey, G., and Aronson, E., (eds.) Handbook of Social Psychology, New York: Random House, 3rd edition.

Morey, Nancy and Luthans, Fred 1985. "Refining the Displacement of Culture and the Use of Scenes and Themes in Organizational Studies", Academy of Management Review, 10:219-229.

Morey, Nancy and Luthans, Fred 1984. "An Emic Perspective and Ethnoscience Methods for Organizational Research", Academy of Management Review, 9:27-36.

Morgan, Gareth and Smircich, Linda 1980. "The Case for Qualitative Research", Academy of Management Review, 5:491-500.

Noesjirwan, Jennifer and Freestone, Colin 1979. "The Culture Game: A Simulation of Culture Shock", Simulation and Games: An International Journal of Theory, Design and Research, 10:199-207.

Ouchi, William G., and Wilkins, Alan L. 1985. "Organizational Culture”, Annual Review of Sociology, 11:457-83. 
Orbach, Eliezer 1979. "Simulation Games and Motivation for Learning", Simulation and Games: An International Journal of Theory, Design and Research, 10:3-21.

O'Keefe, Daniel J. 1990, Persuasion: Theory and Research, Sage Publications.

Palmerino, Mark Langer, Ellen and McGillis Daniel 1984. "Attitudes and Attitude Change: Mindlessness-Mindfulness Perspective”, (Chapter 9) Attitudinal Judgement, Eiser,J. Richard (ed), Springer-Verlag.

Peters, Tom and Waterman, Robert, In Search of Excellence, Harper and Row, 1982.

Petty, Richard, Ostrom, Thomas M. and Brock, Timothy C. 1981. Cognitive Response in Persuasion, Lawrence Erlbaum Associates.

Pierfy, David 1977. "Comparative Simulation Game Research: Stumbling Blocks and Stepping Stones", Simulation and Games: An International Journal of Theory, Design and Research, 8:255-268.

Plummer, Charles M. 1982. "Future School Simulation / Game: Changing Attitudes with Counterattitudinal Role Playing", Simulation and Games: An International Journal of Theory, Design and Research, 13:92119.

Pondy, Louis R., and Mitroff, Ian I. 1979. “Beyond Open Systems Models of Organization", Research in Organizational Behavior, 1:3-39.

Powers, Richard B. 1989. “ZAN-TEC's Game”, P.O. Box 307, Oceanside, OR 97134.

Pratt, Linda and Uhl, Norman 1977. "Evaluation of Games as a Function of Personality Type", Simulation and Games: An International Journal of Theory, Design and Research, 8:336-346.

Preston, N., Hudson, B., and Thios, S. 1976. "Educational Cognitive Style as a Factor in Learning through Simulation", Paper presented at the Fifteen Annual Conference of the North American Simulation and Gaming Society, Raleigh.

Reardon, Kathleen K. 1991. Persuasion in Practice, Sage Publications.

Reimanis, G. 1971. "Effects of experimental IE modification techniques and home environment variables on $\mathrm{IE}^{n}$, presented at the American

Psychological Association Convention.

Rokeach, Milton 1979. Understanding Human Values, The Free Press, Macmillan Publishing Co. 
Ross, Lee and Anderson, Craig, 1982. "Shortcomings in the attribution process", in Kahneman, Daniel, Slovic, Paul, and Tversky, Amos, (eds) Judgement under Uncertainty: Heuristics and Biases, Cambridge University Press.

Sashkin, Marshall, and Burke, W. Warner, 1987. "Organization Development in the 1980s", Journal of Management, 13:393-417.

Sashkin, Marshall, and Fulmer, Robert 1985. "Measuring Organizational Excellence Culture (with a validated questionnaire)", Paper presented at the Academy of Management Organization Development Division, San Diego California.

Schein, Edgar 1985. Organizational Culture and Leadership, Jossey-Bass Publishers.

Schild, E. O. 1968. "The Shaping of Strategies", in Simulation and Games in Learning, Boocock and Schild (eds) Sage Publications.

Schwartz, Howard and Davis, Stanley 1981. "Matching Corporate Culture and Business Strategy", Organizational Dynamics.

Sculley, John, with Byrne, John Odyssey, Harper and Row, 1987.

Shelton, Mary Lou and Rogers, Ronald W. 1981. "Fear-Arousing and Empathy-Arousing Appeals to Help: The Pathos of Persuasion", in Journal of Applied Social Psychology, 11:366-378.

Sherif, M., Sherif, C. W., and Nebergall, R. E. 1965. Attitude and Attitude Change. Philidelphia: Saunders.

Shirts, Gary R. 1976. "Simulation Games: an Analysis of the Last Decade", in Programed Learning and Education Technology, 13:37-41.

Spradley, James P. 1980. Participant Observation, Holt, Rinehart and Winston.

Spradley, James P. 1979. The Ethnographic Interview, Holt, Rinehart and Winston.

Spradley, James P., and McCurdy, David W. 1972. The Cultural Experience, Science Research Associates Inc.

Stein, Barry A. 1980. "Experiencing Organizational Structure", Advances in Experiential Social Processes, Alderfer, C. P. and Cooper, C. L. (eds) John Wiley and Sons 2:245-266. 
Stopp, Peter, 1976. "Understanding, and Evaluating the use of simulations and games in schools", Programed Learning and Education Technology, 13:29-36.

Stotland, Ezra, Mathews, Kenneth E., Sherman, Stanley E., Hansson, Robert O., and Richardson, Barbara Z. 1978. Empathy, Fantasy and Helping, Sage Publications Inc.

Taylor, Shelley E., and Crocker, Jennifer, 1978. "Schematic Bases of Social Information Processing", (Chapter 3) Social Cognition: The Ontario Symposium, Higgins, E. T., Herman, C. P., and Zanna, M. P. (eds), Volume 1.

Thatcher, Donald C. 1990. "Promoting Learning Through Games and Simulations", Simulation and Games: An International Journal of Theory, Design and Research, 21:262-273.

Thornton III, George and Cleveland, Jeanette 1990. "Developing Managerial Talent Through Simulation", American Psychologist, 45:190-199.

Walsh, James P. and Charalambides, Leonidas C. 1990. "Individual and Social Origins of Belief Structure Change", The Journal of Social Psychology, 130:517-532.

Walsh, James P. 1990. "Knowledge Structures and the management of organizations", Unpublished manuscript, Dartmouth College, Hanover, $\mathrm{NH}$, Amos Tuck School of Business Administration.

Watzlawick, Paul (ed), 1984. The Invented Reality (Contributions to Constructivism), W.W. Norton and Co.

Watzlawick, Paul, Beavin, Janet and Jackson, Don 1967. Pragmatics of Human Communication, W.W. Norton and Co.

Weber, Renee and Crocker, Jennifer 1983. "Cognitive Processes in the Revision of Stereotpic Beliefs", Journal of Personality and Social Psychology, 45:961-977.

Weick, Karl 1985. "The Significance of Corporate Culture", Organizational Culture, Frost, Peter', et al., (eds), Sage Publications Inc.

Whiteley, T. R. and Faria, A. J. 1989. "A Study of the Relationship between Student Final Exam Performance and Simulation Game Participation", Simulation and Games: An International Journal of Theory, Design and Research, 20:44-64. 
Williams, Robert and Williams, Sharon Ann 1987. "Level of Identification as a Predictor of Attitude Change", Simulation and Games: An International Journal of Theory, Design and Research, 18:471-487.

Williams, Robert H. 1986. "Changing Attitudes with 'Identification Theory" Simulation and Games: An International Journal of Theory, Design and Research, 17:25-44.

Williams, Robert H. 1980. "Attitude Change and Simulation Games", Simulation and Games: An International Journal of Theory, Design and Research, 11:177-196.

Wright, Jeffrey R. 1977. "Powerplay: A Simulation Game for thi€ Analysis of Complex Regional Energy Issues", Masters Thesis, Department of Civil Engineering, University of Washington.

Young, R. A. 1979. "The effects of value confrontation and reinforcement counseling on the career planning attitudes and behavior of adolescent males". Journal of Vocational Behavior, 15:1-11.

Zaleznik, Abraham 1977. "Managers and Leaders: Are They Different?" Harvard Business Review, May-June. 
APPENDIX A

CLIENT LETTER OF AGREEMENT 


\section{Graphip}

Mentor Craphics Corporation

as00 S.W. Creekside Place

Beaverton, O\& $97005-7191$

(503) $626-7000$

November 3,1986

MI. Terry Schumacher

7907 S.E. Grant

Portland, Oregon 97215

Dear Terry:

The purpose of this letter is to create a written understanding that we have reached concerning the Corporate Culture Simulation project which we have been discussing during our recent meetings. This project will benefit both of us as you will use much of the information in your doctoral dissertation and we will have the benefit of a simulation exercise which we will use in employee and management training related to our corporate values.

PROJECT GOAL

The basic goal of this project is the creation of a simulation exercise which we believe will be effective in teaching individuals the critical attitudes and frame of reference identified as our Company's values and or culture. The exercise will require approximately two to four hours for participants to complete and will be conducted in groups of 10 to 20 individuals. In order to accomplish this goal there are several major activities which need to be completed. These are:

1. Conducting interviews and observing individuals operating inside Mentor Graphics to produce an ethnographic description of our culture. We agree to identify at least 30 individuals to participate in this process in order that you may complete the ethnography. We understand that this may involve approximately 7 hours per person to complete but that the time you spend with any one individual will be spread out over several meetings.

2. Creating and testing for reliability an attitude survey which will measure attitudes along dimensions identified in the ethnography. We are to ensure that a sufficient number of managers (approx. 120) will complete the attitude survey and a later post simulation survey. It will be administered by the HR department in such a manner that it appears unconnected to the ethnographic interviews or the simulation exercise. 
3. Developing materials for use in the simulation exercise such as video tapes, stage props and printed materials. The cost. of producing these support materials will be born by Mentor Graphics while the content, format and medium will be determined and developed by you.

4. Administering the simulation exercise to at least 80 managers at the completion of steps 1-3 listed above. This is to be accomplished within four months of the satisfactory completion of the simulation exercise.

$\operatorname{cosT}$

Terry, you assume full responsibility for the development and testing of the simulation exercise. It 18 our role to supply you with the necessary support (i.e. access to our facilities, and people we identify to participate during the development and the initial use of the simulation exercise as well as the cost of support materials). We agree further to have a minimum of 80 employees complete the simulation exercise at a cost of $\$ 125$ per participant assuming the exercise itself is administered by a representative of Mentor Graphics. The cost for the first 80 participants will be $\$ 175$ per person if we request you to administer the exercise.

Upon completion of the initial phase of Implementation (involves the 80 participants) we will renegotiate the cost and ownership of the simulation exercise you developed at Mentor Graphics.

\section{ACCEPTANCE}

It is your responsibility to produce a quality simulation exercise which meets with our approval before administering the exercise to the initial 80 participants. Upon our acceptance, we will then agree to proceed with the initial implementation. We do not intend to arbitrarily withhold acceptance and will work with you in good faith to correct any deficiencies. Further utilization beyond the initial 80 participants will be at our choice. Should we decide to continue using the exercise we will negotiate a price for outright purchase or a cost per copy of the materials we use.

\section{CONEIDENTIALITY}

We understand that the results of your work and the data gathered in the initial implementation of the simulation exercise will be used in your doctoral dissertation. You will supply us with a copy of the dissertation upon its completion.

Because it is important not to divulge the total scope of your project or its specific purpose, we agree to limit this information to a small select group of individuals. We will work with you to develop a "cover story" announcing your role that will be used as you carry out your work. 
Hopefully, Terry, this letter contains the essential ingredients of our agreement. You recognize, however, that there may be special circumstances unforeseen and beyond our control that would force us to terminate our relationship before the project is completed. This could include, but not limit to, such things as serious economic constraints affecting our Company's financial performance resulting in curtailment of expenses, acquisition by another company, reorganization of the Human Resource function and the like.

It is our understanding that you will receive guidance from your doctoral committee at Portland State University. He will do everything we can to provide your committee with feedback on your project or other information they might request.

To acknowledge our comnon understanding of the project scope and the terms and conditions, it is appropriate that all parties

- involved sign this letter.

Sincerely,

Dick Anderson

Vice President - Human Resources

$D A: d m$

cc: Grethe Larson

$1 /$ errez

Terry Echumacher
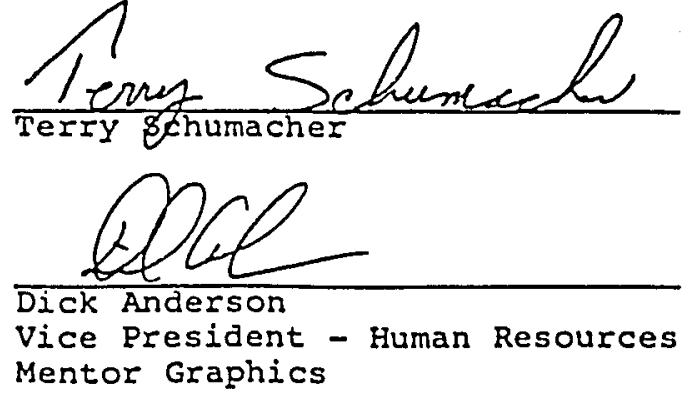

$\frac{11-8-86}{\text { Date }}$

$\frac{11-10-86}{\text { Date }}$

$\frac{\text { Sulgenne }}{\text { Dr. Steven Brenner }} \frac{11-7-86}{\text { Date }}$

Associate Dean for Graduate Programs

Portland State University 
APPENDIX B

MENTOR GRAPHICS CULTURAL TERMS 
This appendix presents a partial list of terms encountered during the interviews of Mentor Graphics employees in Phase 1 of this study. Some of these were integrated into the WINNING AT DESIGN AUTOMATION simulation. The Constructivist perspective that was adopted in Phase 1 places great emphasis on capturing the category system, the "emic" viewpoint, of participants in a social reality to understand that reality. Reading a portion of this list can help convey how people at Mentor Graphics saw that reality as these terms were used frequently in their conversations.

"AA" - Administrative Assistant. A position title that an observer might think is synonymous with "secretary". More responsibility and empowerment are associated with this position at MGC.

Aegis $^{\text {TM }}$ - Apollo proprietary operating system which supports their extensive networking capabilities. (See also Domain, UNIX).

Apollo - Company that manufactures the workstations on which Mentor Graphics sells its software. Apollo headquarters is in Massachusetts, they are primarily a hardware company. Apollo is considered to be the technical leader in network computing. Apollo was acquired by Hewlett-Packard in the spring of 1989.

ALD (Automated Layout Division) - One of Mentor Graphics product divisions. This group develops tools to do physical layout (CAD) the downstream end of Mentor's product line. At the present time ALD is mostly located in San Jose with a small group in Beaverton. (see CADI) There are two major product lines for layout .- the IC layout tools for microscopic scale and PCB tools for macroscopic scale devices.

APD (Advanced Product Division) - A division of Mentor Graphics created in October 1987. This group has responsibility for development of the "core" products (database, libraries, human interface, plotter/printer drivers) which all the applications software use. Prior to the creation of this division this function was located with DAD.

ASIC (Application Specific Integrated Circuit) - During the 1970 s and early 80 s a relatively small number of general purpose ICs were designed and build by companies such as Intel, TI and Motorolla. Most companies bought these ICs and used them as "off the shelf" products. The cost of IC development was spread over thousands or millions of units. With the diffusion of electronic CAE technology in the mid.80s many more companies have begun to design ICs for exclusive use in specific products - hence the name ASIC (a term used less often, though it is more descriptive for people outside the industry, is "custom IC"). These chips are not intended for sale to other companies and production runs are far far smaller than for, say the Intel 8088 or the Motorolla 68000 . This is one of the fastest growing markets for electronic CAE. 
Beat up (as in "I need to beat up Pat this afternoon") - A phase that refers to giving feedback to someone, usually in a one on one setting. The intention is to make the other person understand your point of view on some issue so that it can be resolved or, so that in the future, the person will weight your perspective more heavily in making decisions. The setting is usually not one of conflict or adversity, nor is it necessarily emotional. More than one person was heard to brag "I really got beat up over such and

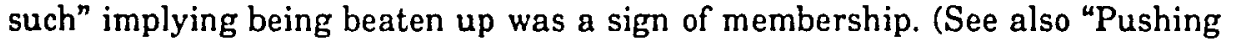
Back".)

Benchmark - An empirical test of a product (or products) in which a customer presents one or more designs and watches as the product seller attempts to complete the design in a "test driven mode. Because CAE tools are complex there can be a long learning curve before users performance is optimal. Benchmarks allow shoppers to see experts put the tools through the paces. Usually difficult (sometimes impossible) designs are selected and performance measures recorded. A benchmark can last as long as a week and involve 20 people.

Beta release - An early release of software to selected customers to elicit feedback about the products features or performance. Beta sites (those customers who agree to test such software) have an early look at and greater influence on new products. The downside is Beta releases are prone to crash and often have limited documentation or support. Their performance can be much slower than the final release.

BLM - Behavioral Level Model. A higher level simulation model in which individual gates or transistors are represented by more abstract components. This abstraction results in some loss of accuracy of results but is necessary to simulate the entire chip in complex designs. See also Gate level model.

Boardstation ${ }^{\top M}$ - A MG product developed by the ALD group for layout of PCBs. This is a CAD tool.

BQR - Beta Quality Release.

Bread board - A prototype of a hardware device constructed to debug the design. CAE tools, particularly simulation tools, were developed to replace this time consuming process.

Build - The process of compiling, linking, binding, etc. the hundreds of programs and subroutines that comprise Mentor Graphics software products prior to test and eventual shipment to customers.

Burn out - As in "Gerry is burned out". Analogy of an electronic component than has had an overload and needs repair or replacement. Refers to someone who has had a high level of work or stress and has lost enthusiasm.

C++ - (Pronounced, "See plus plus") An object oriented programing language adopted by Mentor Graphics in 1987 for use in future software products. C++ is actually a preprocessor for a $\mathrm{C}$ compiler. ( $\mathrm{C}$ is a language developed by Bell labs in the $1970 \mathrm{~s}$. The Unix operating system is written in C. C itself is not an object oriented language). Prior to the adoption of $\mathrm{C}++$ nearly all Mentor Graphics software was written in Apollo Pascal (also not an object oriented language). 
CAD (Computer Aided Design) - That portion of engineering design which addresses physical layout. In electronic CAD this refers to IC layout (micro) and BCB layout (macro). CAD is downstream in the design process from CAE.

CAE (Computer Aided Engineering) - The furthest up stream stage of design automation. This term was coined by Mentor Graphics to differentiate themselves from the already established CAD/CAM companies. CAE includes schematic capture and design analysis.

CADI (California Automated Design Incorporated) - A company in San Jose acquired by Mentor in 1983. CADI had CAD products which complemented Mentor Graphics CAE tools and the acquisition was intended to broaden the market that Mentor Graphics was selling to. CADI became the ALD division when Mentor Graphics divisionalized in 1984.

CAP - (Computer Aided Publishing). - That segment of the Design Automation industry concerned with document management and production. Desktop publishing is the low cost, low integration end of this industry. Context Corporation was Mentor Graphics' entry into CAP, an industry which could grow to be several times the size of the electronic CAE industry where Mentor Graphics competes. Context did not see themselves as competing in desktop publishing business, a lower end market segment. Context was reabsorbed by Mentor Graphics in 1987. Mentor Graphics effectively exited this market in 1991.

Capturestation ${ }^{\mathrm{TM}}$. A MG product developed by the $\mathrm{DAD}$ group for entry of schematic diagrams by engineers early in the design cycle. This is the CAE tool furthest upstream in the design cycle.

CASE (Computer Aided Software Engineering) - An industry segment within Design Automation which remains immature. The idea of CASE is to automate more of the software development process to increase the productivity of software engineers. Companies like Apollo, Mentor Graphics and Context saw this as a future opportunity for growth that could evolve out of tools that they are developing to support production of their software products. In April 1988, Mentor Graphics purchased TekCASE, a division of Tektronix that sells several CASE products. Mentor Graphics exited this market in 1991.

CAT (Computer Aided Test) - Computers have been used to test ICs and boards for nearly a decade (before the development of CEA). This industry segment is lead by HewlettPackard, GenRad and others because it is driven by the hardware involved in the testing process. In the mid 1980s Mentor Graphics developed a product (HVS) that could do some of these functions and they almost merged with IMS in 1987 to broadened their product offering into this segment. Mentor Graphics no longer competes in this market.

CE (Compute Engine ${ }^{T M}$ - Hardware product designed and sold by Mentor Graphics to accelerate compute intensive tasks. The $\mathrm{CE}$ is a pipelined parallel processor 6 CPUs) that can have from 20MB to 192MB of RAM memory. The concept behind CE was "global acceleration", having hardware designed to accelerate tasks across the spectrum of engineering design. In contrast, nearly all accelerators sold today are "point" accelerators and speed up only one task out of many that comprise engineering design. The CE had mixed success in achieving its design goal of 
global acceleration. Mentor Graphics acquired Synergy Data Works in July 1984 for the purpose of developing the CE. CE first shipped in Q4 1986 and the CSD division that built it was disbanded shortly thereafter. The late completion of the $\mathrm{CE}$ and its lack of market success caused Mentor Graphics to return to their early vision of concentrating on software and avoiding hardware.

CELLstation ${ }^{\mathrm{TM}}$ - A MG product for layout of integrated circuits built up from standard cells. This is a CAD tool.

Change Control ${ }^{\mathrm{TM}}$ - A Context product that allows multiple users to work on a single document simultaneously and retains the ability to recreate any of the past versions of the document. A major idea in this approach is "inclusion by reference".

CHIPstation ${ }^{\top M}$ - A MG product developed by the ALD group for physical layout of full custom integrated circuit designs after they have been verified by simulation and rule checking. This is a CAD tool.

CHIPgraph ${ }^{T M}$ - A MG product developed by the ALD group, it is the graphics editor for IC layout. Along with Remedi ${ }^{\mathrm{TM}}$, it is a subunit of CHIPstation ${ }^{\mathrm{TM}}$.

Code chill (freeze) - the partial (and final) fixing of the computer code for a specific release to allow those working downstream a stable base upon which to do their tasks (integration, test, etc).

Compiler - A computer program that converts a high level computer language "source code" (e.g., FORTRAN) into machine language "object code" so it can be executed by the hardware.

CONTEXT - A company spun off Mentor in 1986 to pursue the Computer Aided Publishing market (see CAP). CONTEXT products grew out of the documentation products (DOC and PIC ED) in the original Mentor Graphics electronic CAE tools. Mentor Graphics exercised their option to "reacquire" CONTEXT in the spring of 1988.

CSD (Computer Systems Division) - When Mentor Graphics originally divisionalized (1984) three product divisions were created, DAD, ALD and CSD. CSD had responsibility for hardware products at Mentor Graphics and produced the Compute Engine, HML and HVS. CSD was located in Beaverton and was disbanded after release of the CE at the end of 1986.

DA (Design Automation) - The industry which contain $\mathrm{CAE}, \mathrm{CAD}, \mathrm{CAP}, \mathrm{CASE}, \mathrm{EPAD}$, CAT. In 1989 Mentor Graphics was the third largest software supplier to this industry behind IBM and Intergraph.

DAC (Design Automation Conference) - An annual conference, the largest in the Design Automation industry. Major product announcements and some releases are timed around this event, usually in June.

DAD (Design Analysis Division) - Mentor Graphics original product division. This group developed tools for schematic capture, and various analytic tools (rule checking and simulation) to test designs. The Falcon project resided within DAD until the summer of 1987 when the APD division was formed to focus exclusively on 
Falcon. DAD was located in Beaverton and was the largest product division until it was divided in August 1990.

DAISY (DAISY SYSTEMS) - Mentor Graphics primary competitor during the company's early days (1981-1985). Daisy was one of "little three" (with Mentor and Valid) who started the CAE industry. Daisy originally sold their own proprietary hardware platform to run their software. They fell on hard times in 1984 after they announced they would port their software to the DEC platform and then failed to produce the product for over a year. Orders were sparse during that year as customers waited for the new software. Many people at Mentor feel Daisy got what they deserved for selling "vaporware".

DN 550 or 660 - Older Apollo workstations made obsolete by the DN 3000.

DN 3000 - Apollo workstation introduced in spring 1986. This machine dramatically limited the chances of success for the Compute Engine

DN 4000 - Apollo workstation introduced in June, 1987 and discontinued about 16 months later when it became obsolete with the introduction of the DN 10000. A classic example of the rapid pace of change in workstation technology.

DN 10000 Apollo workstation introduced in September, 1988. Previously Apollo used the Motorolla $68020 \mathrm{CPU}$ in its workstations. The 10000 is the first multiprocessor box which can have 1 to 4 cpus and these are Apollo designed RISC chips.

DOMAIN ${ }^{\mathrm{TM}}$ - Apollo proprietary token passing ring system which gives them extensive networking capabilities. The Apollo network runs at 12 megabits per second and is the technological leader in networking by a substantial margin. Apollo competitors, such as SUN Microsystems and DEC, use the Ethernet system to link the nodes of their networks.

DRAFTEE - Term that came into use to refer to those people who were relocated within the company because of the pressures of the 8.0 release. The term conveys some sense of how little "in control of our own destiny" some employees felt during the 8.0 project. Prior to then, references to large numbers of employees sometimes used the phrase "the troops", a term which they did not appreciate as it made them feel like part of a faceless mass.

DSEE (pronounced "DIZZY") - A software development environment produced by Apollo which was considered to be an early prototype of a CASE tool. Mentor Graphics was the most intensive user of this environment and brought most of their products "under Dizzy" in 1987.

Dwell - A term used to refer to the length of time between shipment of a new software release and the actual installation of the software by customers. Various people at $M G$ give different estimates of the size of this phenomena (and it fluctuates greatly from release to release) but 3 to 6 months is a commonly mentioned value. When dwell is long compared to inter-release periods, customers can become confused and field support is made much more difficult because several different generations of each product are being used, each with different support requirements. 
ELP (Entry Level Product ${ }^{\mathrm{TM}}$ ) - A MG product for schematic capture that was designed to run on the IBM PC and thereby give Mentor Graphics a toe hold in the low end of the business ( ${ }^{\text {C }} \mathrm{CAE}$ for under $\$ 10,000^{\prime}$ ). This is a CAE tool that was put on the "back burner" (no more enhancements) in 1987. Mentor Graphics did not believe that their market would migrate to the PCs (lower performance boxes than workstations) and this product implied a loss of focus.

EPAD (Electronic Packaging And Design) - A segment of the design automation industry, defined by Mentor Graphics, that is downstream of physical layout (CAD). It addresses the placing of boards and other components (including cables) into a physical cabinet, and the analysis of thermodynamic and vibration related properties. Mentor Graphics formed a new division to develop products for this market in 1985 and product introduction was in first quarter 1988. Some felt that this was a step toward Mentor Graphics entering the mechanical design automation market, one that is much bigger that electronic design automation.

FAE (Field Application Engineer) - A engineer who visits customers to help them with problems. They often assist in sales work, training, demos, etc.

Falcon - Mentor Graphics second generation software product had several names during its evolution. It began as a "Port project" and was quickly named "V2". It became "Falcon" during 1987 with the formation of the APD division. Later Falcon became ${ }^{4} 8.0^{n}$.

"Finger pointing" - In the ideal role model, people at MGC took responsibility for mistakes. They said, "I'm sorry, it won't happen again." This was commonly observed at MUG and other scenes. "Finger pointing" refers to placing the blame on others or circumstances beyond one's control. It has a negative connotation. It is representative of a more external locus of control.

Float - As in "to float the software". Allowing applications software to be executed from more than one node on a ring. See Node Locked.

FUD - "Fear Uncertainty Doubt", a market situation created by poor competitors who deliver poor products, over promise capabilities, etc. This condition makes selling more difficult for Mentor Graphics.

"Garbage collection" - Memory de-allocation. A function in which software 'cleans up after itself by releasing RAM so that the Operating System can assign it to other functions or applications.

Gate - A component at the basic level of electronic design.

Gate Level Models - The most primitive level of simulation for electronic circuits. Large designs cannot now be simulated on workstations at the gate level as the run times would take months or even years. Therefore a hierarchy of models has evolved to simulate larger pieces of a design at a more abstract levels. See also BLM. Specialized hardware accelerators exist which simulate designs at the gate level.

Global Acceleration - A technical and marketing strategy behind the Compute Engine. Contrasted to "point accelerators" which are designed to increase the processing speed of a very specialized set of tasks (usually done with hardware). 
HDL - Hardware Description Language. A specialized, high level language for simulation.

HML ${ }^{\mathrm{TM}}$ (Hardware Modeling Library) - A hardware product developed by Mentor Graphics that allows users to plug in an actual device (such as a Motorolla $86020 \mathrm{CPU}$ ) and use the responses from that device in the software simulation of systems. The other alternative would be to construct a software model of the device to include in the simulation, a very lengthy process for complicated devices.

"Hundreds \& hundreds of thousands of dollars, year after year after year..." - Phrase used by the sales force to describe the type of customers they are looking for. "People who will buy hundreds \& hundreds ...". This phrase is sometimes chanted by small groups of MGC sales people in a joking atmosphere. It is sometimes chanted by small groups of MG sales people with great seriousness.

HVS $^{\mathrm{M}}$ (Hardware Verification System) - A hardware product developed by Mentor Graphics that allows the use of an Apollo node to do some of the functions of a logic analyzer. Support of this product was given over to IMS in early 1987 as Mentor sought to minimize its efforts in the hardware business.

IC (Integrated Cincuit - "Chip") - An electric circuit which is etched in silicon. Mentor Graphics original product line was conceived to aid engineers designing ICs. This device is at the heart of the "computer revolution".

IMS. A hardware company located in Beaverton that builds test equipment. IMS has taken over future product enhancements of MGC's VHS. Mentor approached IMS to acquire the company in the fall of 1987 and was seen as a good path to enter the test equipment market. The stock market crash in October 1987 was a major obstacle to the acquisition and while some talks continued after the crash the possibility of a deal was history by Q1 1988.

Inclusion by Reference - A strategy for managing updates to documents which reduces storage requirements and simplifies management of the current version while preserving access to previous versions. The strategy is to maintain a list of pointers that, for each version, "reference" what document parts reside where on the network. This strategy is in contrast to keeping multiple copies of a document which complicates making certain that each person has the most current version of a document.

Installed Base - The total number of still active seats (nodes) a manufacturer has sold. (Called "saturation" in the marketing lingo of some industries.) see run rate.

Instantiate - T'echnical term used in Mentor Graphics initial product release. Later referred to as an example of the company's overly technical "we know best" attitude that sometimes put an added burden on customers. A simpler term was used by Mentor Graphics' competitors to refer to this.

Iron (as in "IBM is selling iron") - Computer hardware.

"Low hanging fruit" - Easily captured benefits. For example, the culture teams created in 1991 identified and went after the "low hanging fruit" to achieve a quick impact. 
"Maggs Casual" - Reference to the dress of Brain Maggs, VP of North American Sales. It includes a crew shirt and dress slacks.

Mixer-Term used to refer to a large scale social gathering at Mentor Graphics. Mentor averaged more than one mixer per month during the interviews in 1987-88. Usually there is some theme (Halloween, recent product release, end of summer, new building). Food and often music are provided and frequently there is some organized activity (balloon toss, volleyball, gambling, skiing, dancing). Mixers usually begin at $3: 00$ or $4: 00 \mathrm{pm}$ and run until all the people leave.

MOC (Mentor Operating Committee) - A group of about 15 vice presidents and managers who met every Monday during the interviewing process to conduct the companies business. In this forum they exchanged information and made some of the decisions facing the company such as the release schedule, inventory management, new directions to take in service or training, etc. The MOC has been discontinued.

MUG - Mentor Graphics Users Group which has an annual meeting in Beaverton in March. Customers both compliment and criticize Mentor Graphics during this three day event. Mentor provides their vision of where products are going (in terms of features, technology, etc) and there is a substantial amount of face to face contact between users and Mentor employees.

Net - The set of linkages (logical or physical connections) between elements in a circuit are referred to collectively as a "net". Hence the term "netlister" for a software program that extracts the list of links from a design, and the product "NETED" which is an editor to modify such a list.

Network - A group of workstations that are electronically linked. This allows sharing of data files, resources, electronic mail, etc. Individual workstations are referred to as "nodes". Networking workstations was an important concept in Mentor Graphics early positioning as they were selling to customers who had always run applications in a mainframe environment. The Apollo network at Mentor Graphics Beaverton had about 350 nodes in 1988.

Network Licensing - Issue that became important in 1988 with advances in network technology. Prior to that time each Apollo workstation had the copies of various MGC software that a person using that node could run. It became technically feasible for a company to purchase fewer copies of those products and share them across the network. This was perceived as a major threat to the pricing and sales aspects of MGC's business plan.

"No-Brainer" - A term used to refer to an extremely simple decision, one that anyone should get right and/or very little time should be spent on it. Question -- ${ }^{n}$ would you prefer to be given five dollars or ten dollars?" Answer - "That's a no-brainer", meaning even without a brain a person could make the correct decision.

Node - An individual workstation, also called a "seat" by the salesforce. The term comes from the idea that each workstation is a node on a network. Engineers prefer the term "node" while the sales force more often uses the term "seat".

Node locked - Software that is modified so it can run only on a specific piece of hardware. 
Non-linear (as in "Frank will go non-linear when he hears that.") - A term which indicates the individual has exceeded some limit, caused by unusual input, and is likely to display unusual, extreme or unpredictable behavior. The term comes from the fact that most electrical components are designed to operate under specific conditions and within this range they behave linearly. Outside the range they will fail in unpredictable ways - they go non-linear.

OOD (Object Oriented Design, also "OOP" for "Programming") - The use of object oriented languages and techniques in software design. $\mathrm{C}++$ is an objected oriented language adopted by Mentor Graphics for all future applications development. "The entire industry took a right turn into OOP in 1986." In previous design paradigms, data and the code that worked on that data were isolated into separate files and different memory locations. (For example the "structured design" used in FORTRAN and COBOL.) OOP brings the data and code back together into an "object". This has tremendous implications as multiprocessor hardware emerges. Then parallel processors can simultaneously execute different objects allowing dramatic performance increases.

OEM (Original Equipment Manufacturer) - An agreement by which one company buys a product and resells it bundled into its own products. Mentor Graphics has an OEM agreement with Apollo (who makes the workstations in Mentor Graphic's "systems").

Off load (as in, "my job is to off load Gerry") - When someone has "too much on their plate" (i.e.,, they are over committed) one solution is to have some one else "off load" that person and reduce the workload of the over committed person. An alternative solution is "burn out".

Plate (as in "Marv has a lot on his plate with EPAD") - A term referring to the size of the commitment an individual has taken on. Managers negotiate with their employees about deadlines and try to balance the ability of the individual with "how much is on her (his) plate".

PCB - Printed Circuit Board ("boards"). Macroscopic embodiment of an electronic circuit. (In contrast to an IC, the microscopic circuit device.) Boards are typically green plastic with embeded metal lines to provide electrical connections between the hundreds of components mounted on the boards.

Partnership - Concept of a long term "win-win" relationship with another. Employees at Mentor Graphics often spoke of the company's goal of forming partnerships with customers. The MGC statement of values included "partnerships with our employees" but few interviewees applied the term to relationships with others within Mentor Graphics. The WADA simulation was intended to convey a richer understanding of partnership and its use with others inside the company.

Platform - Another name for a workstation though usually this term refers to a class of workstation such as the "Apollo platform" or the "SUN platform". Platforms are defined by software compatibility so the Apollo DN 3000 and the DN 4000, and the 660 (different models that run the same operating system) are one "platform". Mentor Graphics sold its systems on only one platform, Apollo form its beginning until 1991 when then began to offer the product on SUN. 
POs - Purchase Orders. Final step in a salesperson getting an order, first step in shipping the order.

Port (as in "should Mentor port their software to another platform?") - The process of rewriting a software product to be compatible with different hardware and its operating system. In 1986 Mentor estimated its products represented 3 million lines of code and therefore porting is a major undertaking. With the introduction of the Compute Engine, Mentor Graphics had to port those products that were to run on the CE and this took about one year. In January 1990 Mentor Graphics announced it would port its products to the SUN platform. As of January 1991 this had not happened.

PR8 - Problem Reports. A detected problem with software or hardware. Mentor Graphics developed an elaborate system of recording, addressing and resolving with individual customers problems which the latter reported. Precise statistics on average wait, time to resolution, etc. are tracked.

PUP Program - In mid-1987 Apollo announced that with their next major operating system release (10.0 in mid-1988) some early model Apollo nodes would become obsolete. Mentor had sold several hundred of those nodes years ago. Under the Mentor Graphics sales contract the customer owns the workstation and Mentor Graphics had no contractual responsibility to those customers. However as a good business partner Mentor Graphics announced the PUP program in which customers with those older nodes could trade them in and get a large discount on newer hardware (DN 3000s) and Mentor Graphics would waive the usual charges for software changes. This was a very popular program with customers.

Pushing Back - To question a decision or procedure, to use an "open door" policy aggressively, to convey to another consequences of their actions. During the video tape sessions employees repeatedly referred to the importance of "pushing back" and this led to its incorporation into the WADA simulation. From a perspective of exploring balance in a social system, pushing back is an important negative feedback.

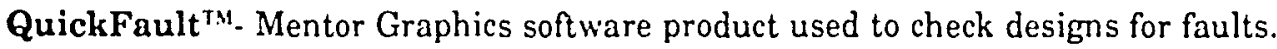

QuickSim ${ }^{\mathrm{TM}}$ - Mentor Graphics software product used for simulating the digital part of electrical designs.

QuickTime ${ }^{\Upsilon M}$. Mentor Graphics software product used for simulating the digital part of electrical designs to check for timing errors.

Release - The updated version of software products. Also the process of preparing and shipping that software. For example Mentor Graphics shipped release 5.3 in April of 1987 and shipped their next release, release 6.0, in September 1987. Mentor Graphics had 2 to 5 releases per year in the years prior to 1987 . There were some customer complaints about this. In 1989 Mentor Graphics undertook a goal of scheduling only 2 releases per year.

Not all products "participate" in each release. For example CHIPgraph may not have enhancements in 5.3 because major work is underway for 6.0 (pronounced "six dot oh" or rarely "six point oh"; never "six point zero"). Newer products or those with major competition are evolving faster and are pushing for more frequent releases. 
Run rate - The number of seats (nodes) a manufacturer has sold in the current time period (usually the current year). (In some industries this is referred to as "penetration" or marginal sales) Run rate and "installed base" can refer to specific applications (such as Chipstation) as well as to nodes. (A node can have multiple applications and could be counted in the run rate of some applications but not others.) see Installed base.

Sales funnel- A technique used by the sales force to structure leads, prospects, and POs so as to focus sales resources and forecast revenues.

Seat - (see Node, Workstation).

Service Bureau - A class of businesses that design boards on a contract basis. They usually do overflow work or very difficult boards.

SGML - Standard Generalized Markup Language. A standard for electronic documents. The DOD has stated that all military contracts must meet SGML by 1990 . Context software products now meet this standard.

Silicon compiler - A technology which held the hope of allowing IC design without the need for experienced VLSI gurus. In 1984-85 it seemed that these hardware products could be designed to accept a specification and produce a "correct" design (one that passes rule checking algorithms). For some applications silicon compilers are used but in general it is considered an immature technology and there are serious doubts how far it will develop. In 1987 none of the leading silicon compiler companies was making money. Mentor Graphics had OEM arrangements with Seattle Silicon and Silicon Compiler Systems.

Specifications (specs) - a document created by engineers that describes a product in sufficient detail that it can be the basis for the work of others on the same project.

Standard Unix - A term used to describe the version (see Unix) that a supplier offers. "Standard Unix" has been labeled an oxymoron by people at Mentor Graphics because the various versions of Unix are somewhat incompatible.

Synergy Data Works - A start up hardware company made up mostly of people who left Floating Point Systems when their ideas for a new computer were not supported by management there. The company included a few from Intel who wanted to build a micro-super computer. Synergy was acquired by Mentor Graphics to build the Compute Engine in July 1984. (see Compute Engine)

Systems Supplier - Mentor Graphics considered itself a "systems" supplier. This means that they provided hardware, software and service as a productivity package to their customers. (See also "unbundled" software.) There are larger margins in selling hardware than software and in the mid 1980s most of Mentor Graphics competitors manufactured hardware as well as software. Mentor Graphics has a OEM agreement with Apollo and gets some mark up on the workstation but this is below what hardware manufacturers make. One person said that with the acquisition of IMS in Q4 1987 Mentor would finally become a "complete company" by adding CAT to their product line, thereby becoming a full systems supplier. See IMS. 
"Tall, thin engineer" - A term used to refer to an ideal engineer, a 'renaissance man' capable of accomplishing difficult tasks under deadline pressure.

TCPIC - A format (actually a protocol) used in communication to a VAX.

Unbundled - Mentor Graphics originally sold only "systems" which included hardware, software and post-sales support. In part this was because customers would pay more for hardware than they would for software. Customers wanted to buy "unbundled" software only from MGC and buy the hardware directly from Apollo. Mentor Graphics resisted this pressure until 1990 when they announced the move to a second hardware platform (SUN) and also offered customers "software only".

UNIX - Operating system developed by Bell Labs in the early 1970s and given to many universities. (Thus several versions exist today, Berkeley UNIX, MIT UNIX, etc.) UNIX is becoming the standard operating system for workstations.

Upgrade - The process of adding functionality to a computer. People also "upgrade" their skills or knowledge".

VALID (VALID LOGIC SYSTEMS) - The smallest of "little three" (with Mentor and Daisy) who started the CAE industry. Valid merged with Telesis in 1987. They bought Calma, a subsidiary of GE in April 1988. Calma has a large installed base ( $>4000$ nodes) of older CAD software. Calma had lost money for years.

"Work around" - Often a problem with the computer code causes it not to perform as intended. (Or the client ases the software in an unforseen manner thereby expecting it to do a task for which it was not designed.) These problems may occur only under certain conditions and therefore not be detected during product testing. As the Company becomes aware of these problems, usually through customer feedback, they determine and publish an interim solution - a work around - to be used until the next software update. (Indefinitely when the product does not evolve to accomplish the task the customer wants it to do.)

Workstation - A class of computers more powerful than PCs but less powerful than most mainframe computers and designed to be dedicated to a sirigle user. Prices range from about $\$ 10,000$ to $\$ 100,000$ in 1987. Mentor Graphics software products are designed to run on workstations and this was a major early differentiator for the company. Prior to Mentor Graphics and its CAE competitors CAD software ran in a mainframe environment. 


\section{APPENDIX C}

ETHNOGRAPHY OF MENTOR GRAPHICS 


\section{ETHN OGRAPHY OF M ENTOR GRAPHICS}

...there is a knife moving here. ...an intellectual scalpel so swift and sharp you sometimes don't see it moving. You get the illusion that all those parts are just there and are being named as they exist. But they can be named quite differently and organized quite differently ... It is important to see the knife for what it is and not to be fooled into thinking that motorcycles or anything else are the way they are just because the knife happened to cut it up that way.

- Robert Pirsig

\section{OVERVIEW}

\section{Purpose of the Ethnography}

This appendix describes Mentor Graphics. It begins with a narrative from a business perspective (products, strategy, markets, earnings) and then presents a description of the Company from the perspective of a visitor to the Company (events, stories, interpretations). The latter is structured, in part, using the Ethnoscience categories described below. This context illuminates the discussion of the frames of reference which guided the development of the simulation class and the questionnaire items that measure attitudes in those frames of reference. In Systems Science a great emphasis is placed on "context" though the methods for how to address this are not explicit. This appendix is one attempt to define the context for the design and use of the questionnaire and simulation.

This ethnographic context is the basis for claims that the attitudes targeted for change in the simulation are linked to important issues in the daily life of those in the organization. It addresses the concern about the "centrality of the dependent variables" mentioned in the literature review the need for some assessment of the centrality within subjects' cognition of the measured attitude change. This claim goes beyond the observation that the leaders of the organization believed the issues addressed by the simulation are important to organizational performance. The claim is outside of the quantitative, hypotheses-testing facets of this study, though the ethnographic context supports a claim for face validity for the measurement instrument that is stronger than if such an extensive grounding in the organizational reality had not taken place. 
The ethnographic context was also provided the raw material for designing the cultural roles and the simulation model. It allowed them to be consistent with many existing attitudes observed in the Mentor Graphics' culture yet display contrast along those attitudes targeted for change. This careful design of the roles was a subtle aspect of simulation design. Because it was constant across all treatment groups - all groups experienced the Beta cultural role as the suggested ideal - it is not addressed by the hypotheses of the study. Future simulation designers will be able to judge for themselves the importance of this approach because the ethnographic context is included.

Finally, it became apparent during the interviews that the culture of Mentor Graphics supports an extremely high level of productivity among employees. As mentioned in Chapter 1 , there is a need for field data about organizational cultures to further our understanding of this emerging research field. The MGC culture may be of interest to those who study organizational culture and productivity as the content of this culture makes it an interesting case study.

\section{Timeframe of the study}

Figure 48 presents the timeline of the study. What is described throughout this study as the "Mentor Graphics culture" is based primarily on the data gathered in the interviews during 1987 and 1988. The culture of Mentor Graphics did evolve during the course of the study and some of those changes are discussed in the final section labeled "MGC culture drift". One indication that the issues defined in the fall of 1987 for the simulation were indeed deep, meaningful, cultural issues is that these issues were still important to the organization in 1990 when the simulation classes were finally conducted and remain important two years later when the Company leased the simulation for use in their training classes. Another indicator was the finding that length of employment at MGC was a significant predictor of attitude change, employees with longer employment tending to have greater attitude change toward the ideals presented in the simulation role. 


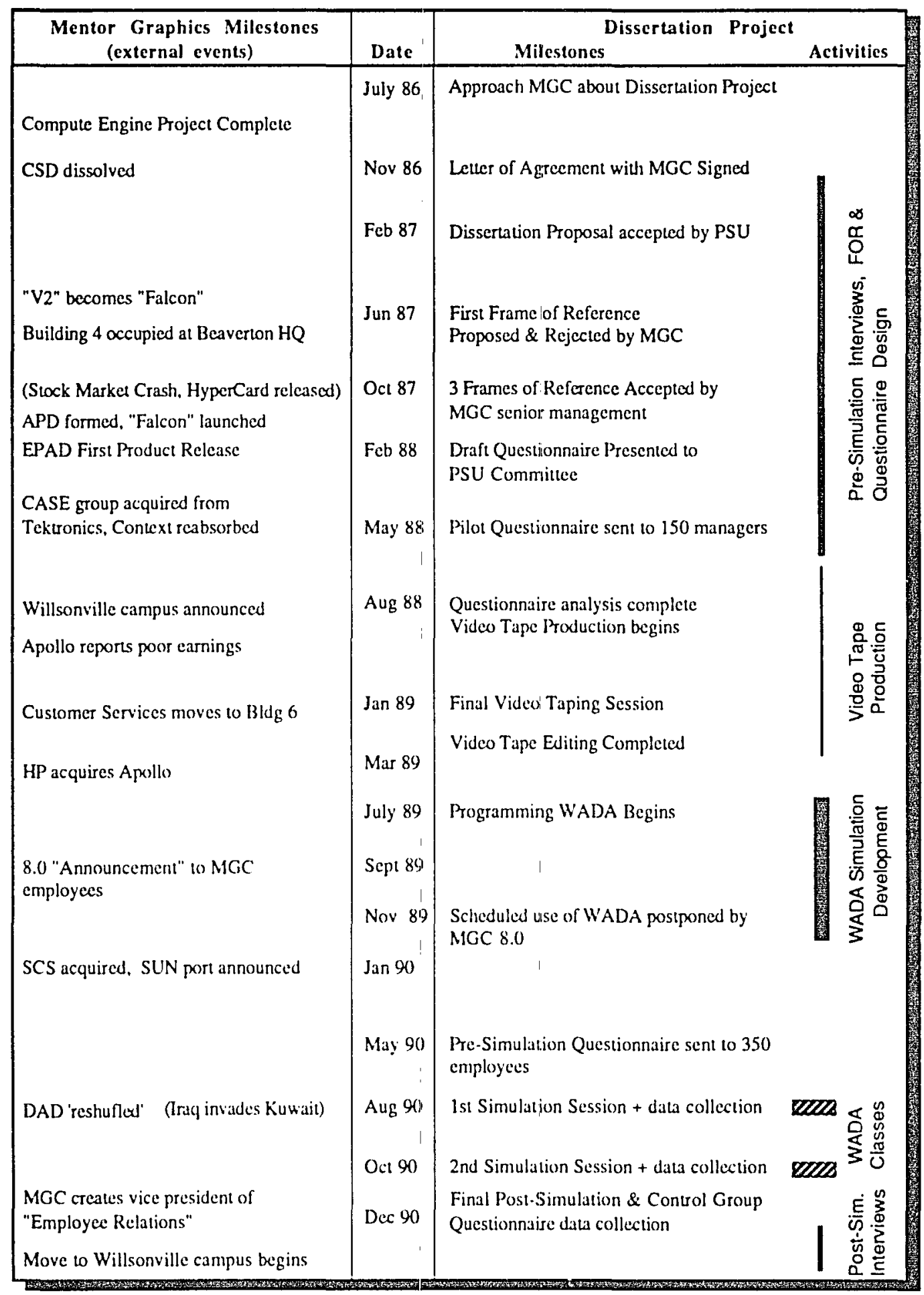

Figure 48. Project milestones and activities. 


\section{ENTOR GRAPHICS - BUSINESS PROFILE}

\section{Founding and Product}

Mentor Graphics Corporation was founded in April 1981. All of the nine early employees had worked at Tektronics. They arranged $\$ 1$ million in venture capital to support the company until the first product shipment in August 1982. Figure 49 below displays the history of Mentor Graphics sales, earnings and employee growth as well as major acquisitions during the 1980 s.

Mentor Graphics "designs, manufactures, markets and services electronic design automation (EDA) systems. By automating various stages in the design process, the Mentor Graphics IDEA Series ${ }^{\mathrm{TM}}$ of Engineering Workstations enables engineers and technicians to design and analyze (computer-aided engineering) place and route (computer-aided design), test (computer-aided test) and document (computer-aided publishing) complex integrated circuits and electronic systems." (1986 Annual Report)

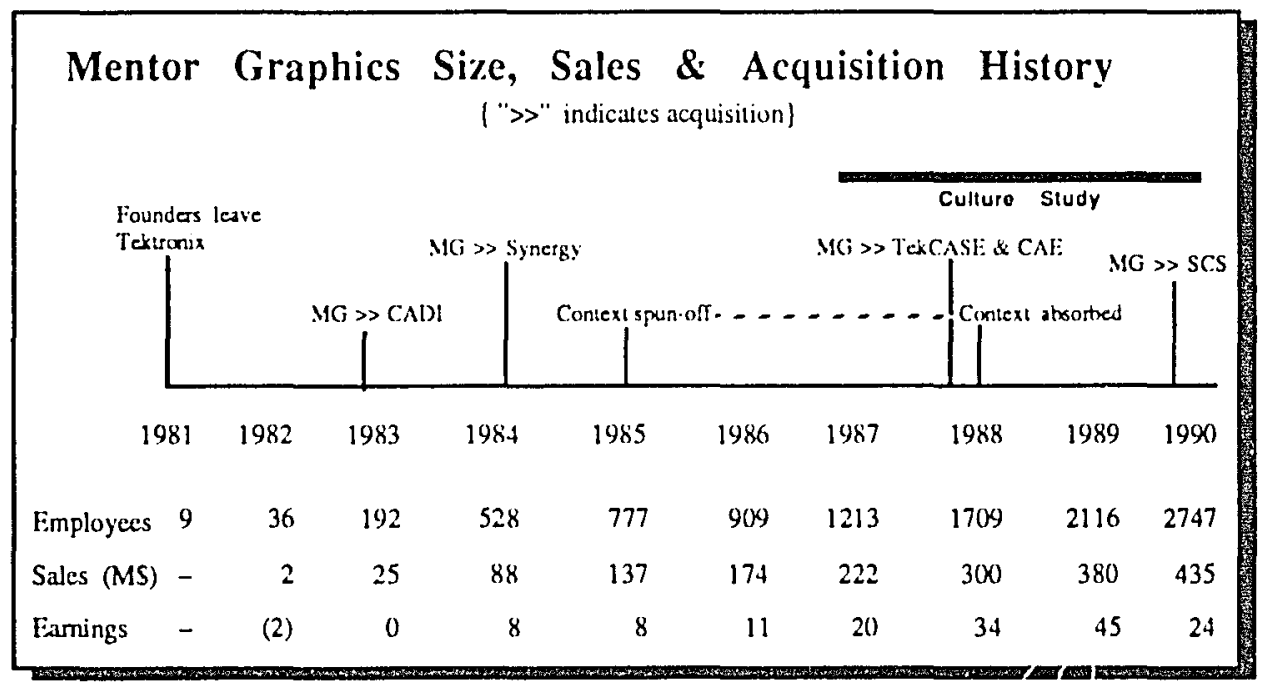

Figure 49. MGC Acquisition, Employment and Sales History.

\section{Initial Vision / Strategy (the First Generation)}

Several facets of the founders early vision became the major tenants of the MGC strategy throughout the 1980s. Several of these differentiated 
MGC from other engineering software vendors and the tremendous success of Mentor Graphics at that time can be attributed in a large part to the fit of this strategy with the environment the company faced.

A major change in the computer market occurred with the introduction of the workstation in the early the 1980s. Prior to that, the established computer design industry was based on mainframe computers. A major thrust of Mentor Graphics marketing was the claim that greater productivity followed from giving engineers greater control over their design environment through the use of workstations - faster turn around, less dependence on computer specialists, and moving from batch processing to real time processing were among the advantages cited. This change also had the financial advantage of allowing software firms to sell many more copies of their products than in the past, a market change similar to that seen for word processors and spreadsheets with the introduction of personal computers.

Software Focus. Mentor Graphics decided not to make their own proprietary hardware, unlike their major competitors Valid Logic Systems and Daisy Systems. MGC's founders did not want to build the hardware for their systems because they believed that customers would prefer to own "general purpose workstations". 'General purpose computers' is a term that is contrasted to "proprietary hardware", hardware which is designed and marketed for a narrow set of applications. (Usually there is relatively little software available for proprietary hardware machines because they tend to have a small installed base and third party vendors are unwilling to port their software to these platforms.) The founders of MGC believed that a general purpose workstation would be easier to sell. They also believed it was easier to differentiate software products. Consider the comment by one MGC vice president, "IBM is selling 'iron" and note the commodity status given to hardware manufacture.

Instead they chose to establish an OEM relationship to buy the hardware, add software and sell the packaged system. In the early $1980 \mathrm{~s}$ the OEM strategy was a disadvantage for MGC because margins were higher on hardware than on software. This left competitors with more profit to reinvest in the rapid growth and product evolution. However the 
MGC vision proved correct in the mid-1980s when customers became more reluctant to buy proprietary hardware. Daisy "nearly died" when they attempted to port their software to another hardware platform. They had been neck and neck with MGC for several years but they were unable to recover market share lost during the port and they faded as a competitor after 1986.

The MGC strategy was to offer their products only bundled with Apollo hardware. They stayed with this strategy for nearly a decade despite considerable pressure from customers, hardware vendors and analysts in the financial community who believed that offering to sell software only or moving onto more than one hardware platform would give MGC more market share and greater appeal. Alternate hardware candidates included Sun, Dec, IBM, HP, Apple and Next. Tektronix and Intellicorp are examples of companies with strategies of providing engineering software on multiple platforms and neither was successful. The leaders at MGC believed that the company would lose focus and product quality would diminish if more effort were devoted to maintaining the software on more than one platform. "You only ride one horse at a time" senior managers would say, "and you must be careful when you change horses."

Product Quality. High quality product and high quality service were things that Mentor Graphics always considered critical to success. Three examples indicate the importance of quality.

The founders held a deep belief that Tektronics (a hardware company) would never compete successfully against them because, "Tek never understood software support". Mentor Graphics established an 800 number and a modem number for customers who had problems. Service goals measured in hours how long customers waited before they received a response.

MGC expanded to overseas markets early, (10 overseas offices by 1984,20 by 1986) but almost always made the extra investment in time and money to create a subsidiary rather than going the route of having a distributor represent them. ('This strategy was in contrast with that of Daisy and Intellicorp, both of whom did poorly overseas). The reason given for this was that the quality of product support and customer service would not be consistently high enough with a distributor (who was less dedicated than an employee). 
MGC was always Apollo's biggest customer but found the quality control on their workstations did not meet Mentor Graphics' high standards. Mentor Graphics developed an inhouse testing system that tested every workstation that they shipped to reduce hardware problems for their customers. This was much more expensive than shipping computers from Apollo manufacturing in Massachusetts directly to MGC customers. Employees in MGC's operations group joked that they were in fact Apollo's Quality Assurance department.

Numerous stories exist about the MGC sales force not selling a product which was available when they believed it was of insufficient quality. One commented that early after its introduction, the Mentor PCB product (Boardstation) was inferior to the competitors' product. "Yeah, I passed up a quarter-million dollar order because I didn't feel I could sell Boardstation in good faith". The Compute Engine also failed to earn the support of the sales force.

One employee related the following story, "how Mentor Graphics found quality", addressing "quality" within the narrow domain of product testing. The engineers had pronounced an early release (circa 4.0,1984) ready to ship but the quality control group (then small) disagreed. After a confrontation the decision was made to ship it - there was considerable market pressure because competitors were shipping improved products. A few weeks later, as customers began to use the new software, the problem reports and complaints overwhelmed customer support. There was a lot of pain. The quality control group was expanded and new procedures were established that made product testing more detailed and more comprehensive. The quality group decided when the product was ready to ship in the future.

Marketing Approach. MGC early adopted a "major accounts", sales approach which focused on building long term relationships with large customers. (These were described as "Partnerships", a concept that will be discussed in greater detail below.) One marketing manager spent six months at a customer's site to better understand their needs for design tools. On another occasion, the Texas division of a customer had adopted MGC software a year ahead of the San Jose division. A MGC engineer who had worked with the Texas division flew to San Jose to present a class and work with them for a week as they came up to speed on MGC's software. 
During breaks in meetings of the sales staff, someone would frequently ask, "What kind of customers do we want?", and the joking response was a chorus of "Those who spend hundreds and hundreds of thousands of dollars, year after year after year." This popular phase indicates both the size and long term relationship that Mentor Graphics sought in their customers.

Another central theme of the early Mentor Graphic's vision was to provide a broad spectrum of integrated products. These included schematic capture, design and parts database, simulation, layout (for IC and boards) and documentation. Most of the company's competitors were focused on one or a few of these market segments (for example, selling only simulation or layout products). This difference in market strategy made comparisons of market share or revenues difficult to interpret. Mentor Graphics sold customers on the idea that this integration of products provided enhanced productivity.

\section{Mentor Graphics' Apollo "marriage"}

Once the decision was made not to make hardware, the selection of the hardware platform became a critical decision. Several employees commented that this was the most critical decision made in the company during its first few years. The Mentor Graphics Story a booklet written by Rich Brewer of MGC's Public Relations department, emphasizes that this decision was delegated to to the engineer with the most technical expertise. This is a good example of an ideal - that many decisions in the company are delegated - that Mentor Graphics has often presented in public forums (e.g., 1985 annual report). It is consistent with the central theme of the "small company atmosphere", giving employees control over their own destiny. There were many instances where the Company has failed to live up to this ideal, but the first and most critical decision in the early years was delegated.

The relationship between Apollo Computer and Mentor Graphics was crucial to the success of both. Apollo was also a start-up in 1981 and Mentor Graphics selected them as their hardware vendor before Apollo had shipped a single computer. The decision proved to be an excellent one as 
A.pollo's networking operating system (Domain ${ }^{\mathrm{TM}}$ ) was technologically superior to all other workstations during the 1980s.

Throughout this period the public face of the relationship between Mentor Graphics and Apollo was one that indicated commitment and teamwork. This was certainly a true picture but an incomplete one. Many employees referred to the relationship as a "marriage" - stormy, stressful, disappointment filled, yet surviving because of commitment and shared benefits (market success). Among other things, Mentor Graphics was unhappy with the poor public relations of Apollo, the low quality of product testing, and poor hardware support.

The fact that it took until 1990 for MGC to announce that they had selected SUN Microsystems as their second platform can be seen as evidence of the commitment to Apollo. During the last half of the decade the biggest strategic issue facing MGC was when (if ?) they should select a second hardware platform, and which one. There was considerable pressure from customers, other workstation manufacturers and voices with MGC to port their products onto another platform. Mentor Graphics' management resisted this for years. Finally, when Apollo reported low earnings in the second half of 1988 they reluctantly decided it was time to change.

The years of delaying this decision can also be given a "cultural interpretation" consistent with the belief that major events shape the culture. Mentor Graphics' management had seen their then biggest competitor (Daisy) "almost die" in porting products onto a second platform (DEC). MGC had also started a port project in response to customer pressure. Within a few months the managers of Mentor Graphics' port project recognized the enormity of that undertaking and this confirmed that the Daisy experience was not only due to mistakes. (The MGC project was refocused as "V2", discussed below. It was not announced publicly, a key ingredient in Daisy's problems.) Stories of Daisy's problems and those of other companies that failed in bringing a new product to market (e.g., Apples "Apple III" and "Lisa") were told again and again by MGC senior managers in the mid 1980s. Beliefs about the danger of porting led them to 
postpone this decision for too long and MGC was able to postpone, but not avoid, the problem of porting their software.

The decision to abandon the strategy of a direct port of the MGC software involved an example of "Pushing back", a cultural phase described in more detail below. At the time there was considerable pressure to complete this project. When IBM announced their "RT" workstation many customers believed that they would sweep aside the competition (Apollo, SUN) like they had in the PC market. Those customers did not want to buy Apollo computers because they feared making the wrong technological choice for their engineering functions. MGC could not sell their software unless customers would but Apollo computers. People involved in the port project questioned, and ultimately reversed, the decision to port MGC software products. (IBMs workstation turned out to be a non-event because the product was never technologically competitive and that was a requirement for workstations, unlike the $\mathrm{PC}$ market.)

\section{Compute Engine}

The Compute Engine (CE) project ended a few months before the beginning of the interviews in Phase 1 . The project was discussed by many employees in the interviews and was the focus of the 1985 annual report.

In 1984 a group of engineers left Floating Point Systems to build a mirco-super computer. They formed Synergy Data Systems and approached Mentor Graphics, among others, to see if MGC would like to purchase the computer they intended to build. Mentor Graphics decided to acquire Synergy and get into the hardware business. MGC divisionalized to accommodate the new people, Synergy became CSD (Computer Systems Division) and the existing software group in Beaverton became the Design and Analysis Division (DAD).

Figure 50 was drawn during one interview as the project history was explained to the researcher. The interviewee labeled the Y-axis as "team spirit" and "feeling of self worth" for the CSD group. Another good label would be internal $(+)$ to external (-) locus of control, a cultural theme further discussed below. 


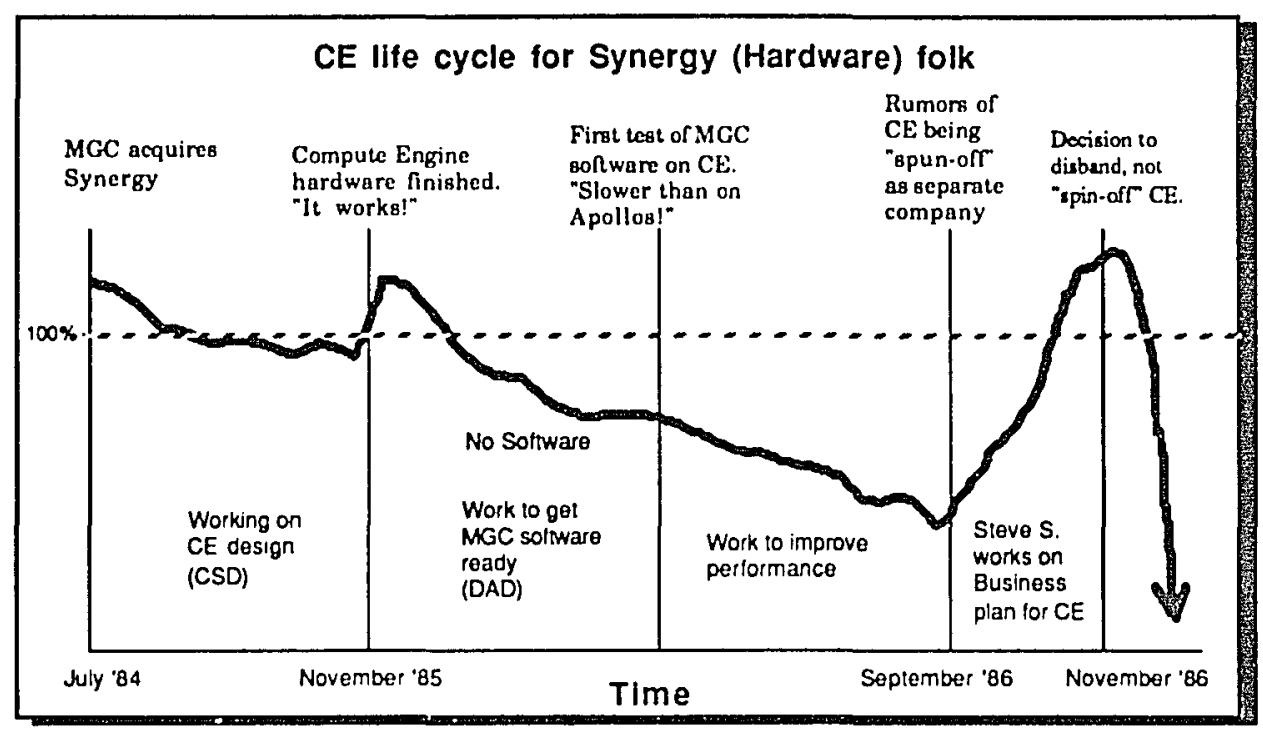

Figure 50, Life cycle of the Compute Engine project.

The Compute Engine (CE) was intended to greatly accelerate the compute-intensive processes of MGC software products. (Many simulations could take hours or even days to execute. Many others were not even attempted because of the extended compute time.) The hardware was designing using MGC software and was finished on schedule. However the software for the $\mathrm{CE}$ was being developed by another group $(\mathrm{DAD})$ and they had other projects that were given higher priority. When the software was initially completed execution speed was marginally faster for some tasks but actually slower for others. A lot of additional work went into rewriting the compilers and the products. Eventually "acceptable" performance was obtained but this was a year later than planned. About this time Apollo released their DN 3000 which was much faster than previous workstations. The market window during which the $\mathrm{CE}$ could have stared had closed.

The Compute Engine was MGC's biggest setback up to that time. They had missed an opening on a large market, and the decision to break up the CSD group (several were terminated, some left on their own, others were scattered around the Company) kept Mentor Graphics out of the hardware business. Several observations can be made linking the project and the culture. 
A considerable "We / They" mentality developed between the hardware (CSD) and software (DAD) engineers during the project. Although there had been differences prior to that time, nothing of this magnitude or duration had happened before. (Duration must be considered in the context that at the end of this two year project MGC was 5 years old.) One founder said, "It was like two different cultures, even though they were separated by only a few hundred feet (building 1 and building 3 ) they didn't communicate." Before this project, a strong shared vision had united members of the culture. The Synergy people came in with their own strong vision, but only very late in the project did other MGC groups get on board. It can be argued that size alone (this was the biggest MGC had ever been) led to the lack of shared vision, but the EPAD project which began in late 1986 and shipped in early 1988 was able to create a strong vision that was well integrated with other groups in the company.

The point remains that the shared vision was absent and the response of the organization could not overcome that condition. The project outcome did feed management's emphasis on "execute - get it done - $50 \%$ of winning is just showing up" which was certainly strengthened by this experience. In typical Mentor Graphics fashion, the company learned from this mistake. They took over hardware service, first for themselves and then for all their customers and this new, profitable business had a base in the parts warehouse developed for the CE. The MRP system established for parts and manufacturing enhanced the "continual improvement" and quality themes of the culture.

The "field" (sales force) did not push the CE as they were uncertain about its value to customers, which indicated their commitment to customers. The CE was repositioned as a "capacity machine" for doing very large designs and though some customers liked it, it did achieved a small fraction of its potential.

\section{"PUP" Program - Commitment to Customers}

Mentor Graphics carefully constructed their early contracts so that customers owned the hardware but only leased the software. This pushed the risk of hardware obsolescence - a constant factor in purchase decisions 
- onto the customer but allowed MGC considerable control over the software. In 1987 when Apollo announced that their next operating system release (10.0) would make early models obsolete, Mentor Graphics decided that their commitment to customers required them to create the "PUP" (Priority Upgrade Path) program to allow customers to swap obsolete workstations for new DN 3000s. There was a major downside risk for Mentor Graphics here because if all customers who qualified participated (most did) and if that involved them allocating money budgeted for new Mentor Graphics equipment to PUP (where there was no profit for MGC) then the Company could face several quarters of dramatically reduced sales.

Dave Moffenbeir, senior vice president said about the PUP program, "It was obvious SOMETHING had to be done to support those customers. It took about 20 miriutes to run the numbers..." Then a very large effort involving many employees over many months carried out PUP. PUP indicates both concern for customers and how quickly MGC could make a major decision when the "correct" response was largely determined by a corporate value, in this case, customer support.

At MUG in 1988, customers repeatedly expressed their thanks for PUP. In a large public meeting one asked, "What is Apollo doing with all those old nodes?", mistakenly thinking that Apollo was somehow involved (they were Apollo computers). Two Vice presidents from Mentor Graphics stood to point out, "Apollo doesn't have a PUP program. Mentor Graphics has a PUP program. If any of you would like to buy some large paper weights..."

\section{The 8.0 Project (the Second Generation)}

When Mentor Graphics decided to cancel the project to port their software to another platform, the realization that that task would be necessary someday did not die. Instead a more elaborate strategy emerged to solve the problem. In 1986, the with the Company's oldest products almost 5 years old, they could see better ways to write those products. The cleanest approach was to start over from square one and create a second generation of products. These could be written in a language that would 
make porting easier and the design could also make allowances for a future port. A great many things had been learned about these software products and the engineers were anxious to improve these products. The port project became "V2" the second version of MGC software.

Several differences made the second generation more difficult to create than the first. MGC now had hundreds of products and hundreds of customers. These placed constraints on new designs. The balance between new technology and compatibility with existing products and standards need to be decided on issue after issue. Different product groups wanted to innovate in order to make their products successful in the market while the existing MGC strategy of offering a well integrated product line suggested considerable constraints and detailed standards for all products to follow. The second generation was to be all things to all people, truly difficult criteria to satisfy (see Figure 51).

In interviews in early 1987 people asked the researcher if he had signed a confidentiality agreement before they talked about V2. It was expected that the project would be finished late in that year and there was concern about early leakage to the public. The project progressed slowly. The $\mathrm{C}++$ language was selected as the standard for all new MGC development. At series of at least a dozen different people were in change as the Company sought to keep the project moving forward. None of those who gave up the lead role were fired, all continued to work in different capacity within the organization.

In mid-1988 it was realized that V2 was not receiving the priority that it needed as one project within DAD. A new group was formed, the Advanced Products Division, (APD) and they were dedicated solely to what was now the "Falcon" project. There were concerns about the slow pace of the project, but the company was very successful without it and as one person explained, "with the first generation we needed to find water (we would die in 3 days without it), with the second generation we need to find food (we have 30 days)." 


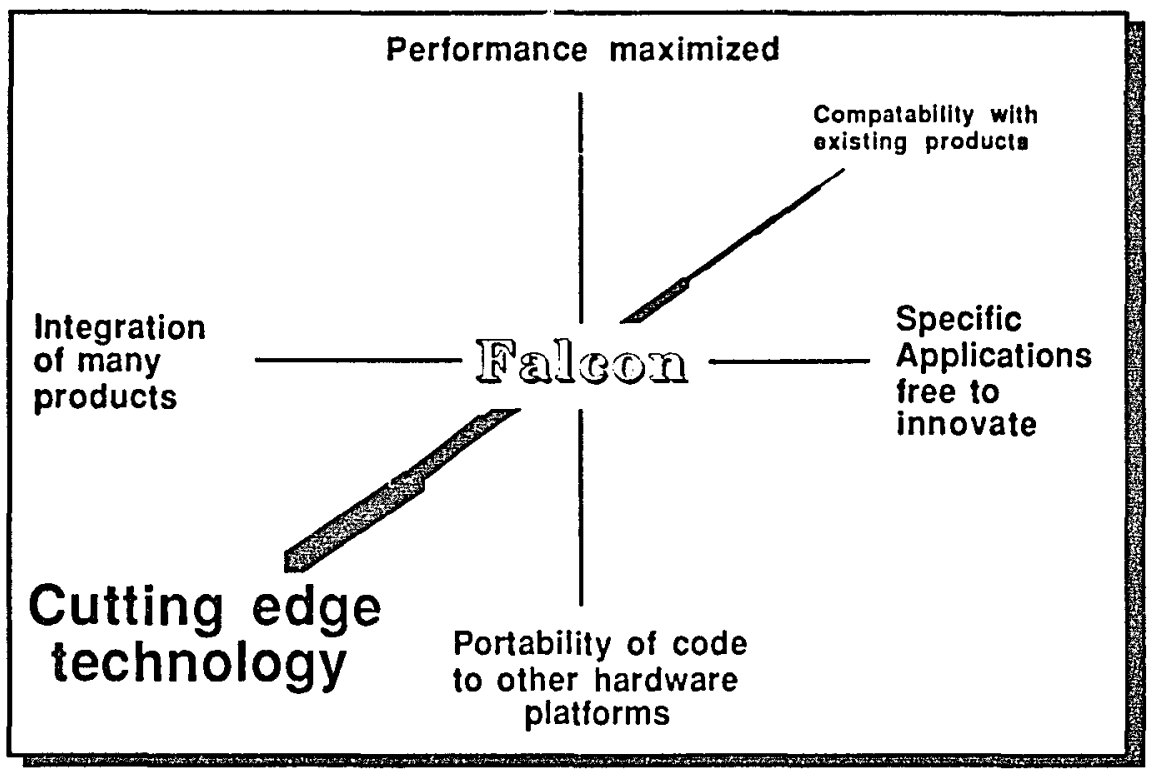

Figure 51. Competing values for the second generation products.

The problems with Apollo as the only platform (acquired in the spring of 1989 by HP, a direct MGC competitor in the EDA software market) placed significantly greater pressure on Mentor Graphics to get Falcon to market because of the expected greater ease of porting. In September of 1989 the "8.0" announcement was made internally. (The last major MGC software release had been 7.0 and so the next, expected in the first half of 1990 , would be 8.0). That announcement had the tone of "batten down the hatches" and bring the second generation to market. Vacations were cancelled, people were told they would be shifted to different jobs in the company (without the normal offering and applying for positions) and all corporate training classes would be cancelled for a year as the company focused on this challenge. (The WADA simulation classes had been scheduled for November of 1989 and the 8.0 announcement postponed its use until the following August.)

The tone of the 8.0 announcement was not characteristic of Mentor Graphics. One employee speaking in late 1989 commented about it, "A lot of MGC people sent resumes to Sequent (a nearby high tech firm) ...the culture and Tom's credibility really went down the toilet." Some managers acknowledged that the announcement did not come close to the ideal way of 
motivating people to accept a difficult challenge. In November, an "8.0 kickoff party was held. T-shirts, hats and bumper stickers proclaimed "I'm working 8.0 days at week at Mentor Graphics" as a huge organizational commitment was made to finish the next release. Lunches would be served in the cafeteria on Saturdays and Sundays.

One hope expressed by managers was that this huge commitment could "rekindle that early drive..." that the Company had in the first few years. But as the project wore on there was a lot of "finger pointing" as deadlines were missed and stress levels soared. During one interview in early 1990 a flyer describing 'Stress reduction classes' that would be offered soon at the Company was shown to the researcher. The footnote indicated "class size would be limited to 25 so sign up early". The interviewee waded the announcement and threw it against the wall. "That's a fucking joke! There are 25 hundred people in this company who need stress reduction."

Attempts were made to make the best of a stressful situation. It was suggested that senior managers make random visits to cheer on those with the heavy burden. This practice was halted however when the interpretation that "they are checking up on us" developed. In early 1990 Mentor Graphics announced publicly that SUN had been selected as the second hardware platform and products would be available "sooner than you think". It was spring of 1991 before major shipment of the 8.0 release was completed.

\section{ETHN OSCIEN CE PERSPECTIVE}

\section{Ethnoscience Categories (A Model of Knowledge)}

Spradley $(1979,1980)$ believes that cultural analysis should address each level in the Ethnoscience hierarchical category system shown in Figure 52. The basic unit of categorization in Ethnoscience is the Domain which Spradley defines as "Any symbolic category that includes other categories.... . Appendix B is a partial list of domains uncovered during the interviews of Mentor Graphics employees. 


\section{Culture the global collection of knowledge shared by a group, can be described as composed of}

Cultural Themes broad, tacit features of the cultural landscape which can be found in several

Cultural Scenes regions in the cultural map, which contain few to many

Domains the primary unit of analysis in Ethnoscience.

Figure 52. The Hierarchical Model of Knowledge in Ethnoscience.

Above the domain, the next level is the cultural scene. The concept of 'frame of reference', adopted in this study to guide the development of the simulation, is similar to a cultural scene in that it is a subsystem of a person's knowledge. As with cultural scenes, each person has many frames of reference within their entire knowledge base. Spradley and McCurdy (1972:26-27) use the analogy of a map to describe cultural scenes.

The various regions of a person's cognitive map are his cultural scenes. Just as a city map can have small sections included in larger ones, which are grouped together into the total area of the city, the cultural scenes that make up a person's cognitive map can vary in size. ...it is important not to confuse a cultural scene with a social situation. The former is the knowledge which actors employ in a social situation; the latter is the observable place, events, objects...

Scenes in the Mentor Graphics culture that were observed during the study are listed in Figure 53. The names given these are taken from the physical setting in which they were observed. It is important to remember the above comment and note that the focus was on how people interacted, what they perceived in the situation, and what things they considered important.

The next level in the Ethnoscience hierarchy is the Cultural Theme. Spradley's (1979:186) definition of cultural theme is "Any cognitive principle ... recurrent in a number of domains and serving as a relationship among 
subsystems of cultural meaning." Themes observed in the Mentor Graphics' culture are the subject of the next section. ${ }^{1}$

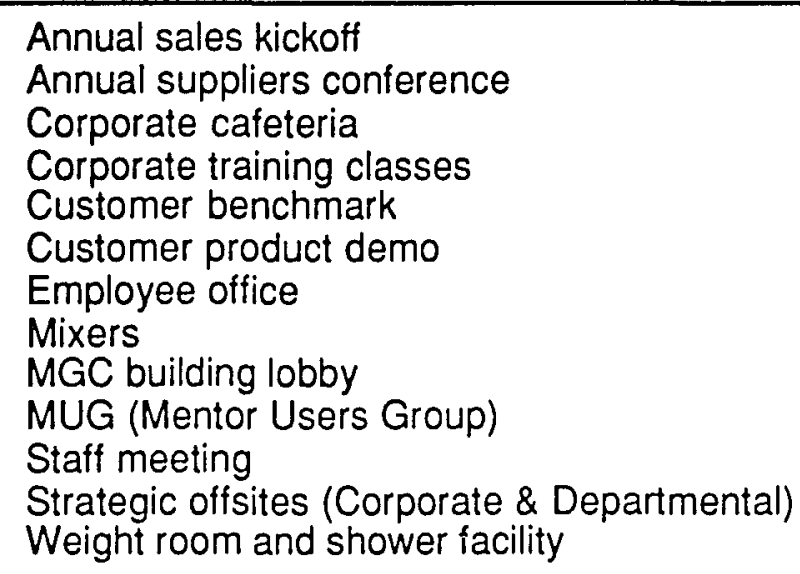

Figure 53. Cultural scenes observed at Mentor Graphics.

\section{THEMES OF THE MENTOR GRAPHICS CULTURE}

The following is a partial list of existing cultural themes at Mentor Graphics that were developed from the analysis of the interview notes.

\section{Where to Begin? - the High Quality of the Peonle}

The culture of $M G$ is complex. It has many facets. Any attempt to describe it can only be partially successful. And this researcher has been exposed to only a portion of that culture which further limits the claim "this is the culture". But putting aside such caveats what can be said?

An early and lasting impression is the consistently high caliber of people throughout the organization. One manager who had come to MG four months earlier offered his initial impressions of MG. His first comment was on the high quality of the people. "I've worked in many high tech organizations and they all have deadwood in them. But I haven't seen

1 It should be noted that the Ethnoscience approach and its model of cultural knowledge are not concerned with attitude statements, the basic unit of measurement used in social psychology. This is related to the Ethnoscience focus on description at the group level of analysis, its emphasis on qualitative description over quantitative measurement and the absence of the Social Psychologists' focus on measuring change. 
any in the four months I've been here. And that surprises me. I'm pretty certain they have some..., but I've already seen most of this organization and I haven't found any yet."

In another conversation a general manager described the near release of a new product for which he had responsibility. Over a year had gone into the design and development of this product. Was he a little nervous? Were there concerns about integration with other parts of the company? He said with a smile, "The rest of Mentor Graphics is so good I know that if I do my job well, then I will succeed. You don't have to worry about your project getting hung up somewhere because of under performance elsewhere in the company." High quality people, high quality processes, and high quality product are a consistent perception one has in learning about MG.

This quote relates that consistently high quality to a theme labeled "locus of control". People at MG expressed a feeling of being in "control of their own destiny" in the sense that their success or failure was dependent on their own efforts. This theme and a related anomaly was to become the focus of the simulation.

\section{"Unique" and Proud of It.}

The overwhelming impression that a visitor gets is that the atmosphere at MG is very positive. The atmosphere is intense. There is rapid change and change is seen as positive by members of the culture. The feeling about the company and the intensity of the activity has been described as 'we're in a race' with our competition. And races are exciting. Races have winners. The people at MG believe their company is and will continue to be a winner. They see themselves as contributing to that success.

Many people I met expressed pride in the "uniqueness of the Mentor Graphics culture". One of the things that was pointed out repeatedly in early visits is the company policy on smoking. Prior to the actual acceptance of an job offer, applicants are required to sign an agreement that they will not smoke in company offices (US offices only). This was mentioned by numerous people in early interviews and was presented as an example of the uniqueness of the company and of how the company is 
concerned about employees and their health. Several people saw this policy as closely related to the provision of exercise facilities in the company offices.

Many employees expressed pride in the culture. They pointed out the smoking policy, the availability of the weight room and shower facilities and the frequent "mixers" (never referred to as "parties' or "social functions") as examples of what made that culture both unique and positive.

One story that is told about the the early days was labeled the "first facilities" story. It recalls how in the very early days when there were nine employees they worked in a small two room rented office. The office had men and women's rest rooms but all nine employees were men and the women's went unused. They soon converted the women's rest room to a shower so that they could go running during work breaks. The story was told several times, always with the conclusion, "MG had exercise facilities from the very early days, this shows that the company cares about the employees".

Another theme which is perhaps more fundamental to the culture is reflected in this story. That theme is "Continual Change". It is clear that the people at MG question things and don't stand on ceremony. They seek new and improved approaches. This style is related to the core technology of the firm, software engineering.

\section{"Continual Change"}

The core technology of $M G$ is software engineering. A brief discussion of that technology makes apparent how a style of work associated with this technology is consistent with the cultural theme of change in the organization.

A decade or more ago, electrical engineers were predominantly hardware engineers. As computers became more complex, bigger teams worked on the design and the building of subunits of those computers. To ensure that the subunits could come together into a functioning system, the engineers developed techniques to plan out in detail exactly how each subunit would work. The engineers wrote a specification document which is like a contract and each major design group signs it to indicate that their portion of the design will perform to that specification. They don't want surprises when many man years of effort can go into each of the subunits before the system can be tested as a whole. 
Software engineers are a completely different breed than hardware engineers. Software engineering has far fewer constraints than other types of engineering. "It is outside of real physical world constraints". The only constraints are the compilers and those are also software. Whereas the hardware engineers devoted a great deal of attention to design procedure, the software folk have an approach that has been referred to as undisciplined, or maverick. They say "Lets try this", and work on it for a while. If it seems to be going nowhere, "they rip it out and start over again".

And this "try it and see, change things frequently" design philosophy of software engineers is consistent with the approach to change throughout the company. People question practices and procedures to see if they can find better ways of doing things. The statement, "this is how we did it in the past" carries little weight in discussions. On several occasions it seemed that people went out of their way to mix things up just to avoid any routinization.

It would be a misrepresentation to give the impression that there is little organization in the design of software at MG. The software MG creates is very sophisticated, millions of lines of code in dozens of applications that are highly integrated and optimized for the targeted performance criteria. Obviously they did not hack their way to such a product offering. In interviews engineers consistently responded "we do a great deal of planning and organizing here. We spend a lot of our time planning". But there is a willingness to change those plans, an openness to try a different approach that is in contrast to more traditional (hardware) engineering cultures.

The pervasiveness of this "willingness to change" theme was highlighted during the creation of the video discussed in the Methodology Chapter. Four of eleven employees made statements to the effect "we need to question procedures or policies and if there isn't a good reason for one of them, then we had better make it go away." They made these statements within the context of discussing their assigned topics of Vision, Coaching, etc. The topic of "Continual Change" had not been included in any of the eleven scripts. But it surfaced in more than a third of the video sessions!

This willingness to change is a recurrent, pattern at Mentor Graphics. I was told more than once that, "We literaliy don't know which building we will be working in next week." The rapid pace and constant change is seen as part of the "small company atmosphere" which the people at Mentor Graphics value. It is not seen as indecisive or waffling but rather responding to new opportunities. There is a widely shared belief that these local changes take place within a guiding context, an overall vision of what the company will accomplish. And there clearly are enduring themes in the culture which guide the company. 
The company vision is a central part of MG culture and a key to how they balance the "chaos" of rapid growth and "Continual Change" against the stability of "organization".

\section{Vision}

"Long Term Vision" was one phase that was mentioned again and again during interviews. The majority of those using this phrase said that MG did have a long term vision and that having such a vision is important for any company to succeed. A few people mentioned having previously worked in companies which had declined because in those cases the vision had been realized but no further elaborated vision emerged. "They just ran out."

A big part of Mentor Graphics' vision from 1982 to 1986 was "beat Daisy, become number one in Electronic Computer Aided Engineering". (People wore 'Beat Daisy' buttons to work.) Charts showing market share in CAE with "the little three" (Daisy, Valid and Mentor) were common in the Company's newsletter, presentations to financial analysts, etc. By late 1986 in was becoming clear that MG had emerged as the dominant leader in ECEA. Both Daisy and Valid had fallen aside. The vision had been achieved.

Early in 1987 company presentations began to show market share for "Design Automation" a more broadly defined industry of which ECAE was perhaps $10-20 \%$ of total revenues. IBM, Intergraph and Computervision were the competitors in this industry, companies with much greater resources and market power than the start-ups Mentor had previously considered its competitors. The Design Automation industry was described as having six subdivisions, (Computer Aided Publishing, Computer Aided Test, Computer Aided Design, Computer Aided Engineering, Computer Aided Software Engineering and Computer Aided Packaging).

When people at MG talked about the vision of the company many mentioned this "six box model". However in discussions with employees present in the early days, one learns that the vision then also included the type of relationships they wanted among employees of the company. The founders of MG consider it to be important that all employees have a good 
understanding of the vision of the company. In the video tape, Berkeley Merchant summarizes the management style of the company as, "give them a vision of where we are going, give them the tools to do the job and let them go." Over time the 'vision' seemed to focus more on revenues and "being a Fortune 500 company" and less on how people would by treated and how they would feel about working for Mentor Graphics.

The problems associated with the Compute Engine were sometimes explained as "a situation where we did not have a shared vision", indicating how $a$ its absence is used to explain disappointing performance.

The evolution of the Mentor Graphics' vision was the subject of an article by Gerry Langeler (1992) who was previously MGC president. That discussion suggests that part of the problem that led to layoffs in 1991 was that the vision evolved to where it no longer focused on "making products that people will buy". A different conclusion is reached by this researcher: the vision evolved to where it no longer included employees "being in control of their own destiny". When that was lost, the level of commitment and productivity dropped significantly. Unfortunately that occurred as the Company entered a lean economic environment. As growth stagnated and the long string of profits was followed by losses, downsizing and refocusing became an alternative.

\section{The "Mavflower" Culture}

One manager described Mentor Graphics as having a "Mayflower" culture. Like the pilgrims on the Mayflower, when they got to the new land they adopted goals and rewarded practices that were in contrast to the lands from which they came. The founders of MGC came from Tektronix and former Tek employees are the single biggest source of MG employees. Several important aspects of the Mentor Graphics' culture can be seen as being a reaction to experiences at Tek. Some of the contrasts between Tektronix and Mentor Graphics that were described during the interviews are outlined in Figure 54.

\section{Hard Work. Intensity and Commitment}

One of the values in the MG culture that can be seen as a response to experience at Tektronix is the high value on "execution", getting things 
done and done quickly. At Mentor Graphics achievement and high levels of output are valued. These are in contrast to "Tek" which is sometimes referred to as "the lazy $T$ ". Stories from former Tek employees describe good products that were cancelled on political grounds and poor products that languished in development for years consuming resources but never generating revenues. A rumor that was repeated several times in the interviews is that the CEO of Tektronix was once asked how many people worked at Tek and he responded, "about half of them". Mentor Graphics employees shake their heads after repeating this story.

\begin{tabular}{|c|c|}
\hline Tektronix & $\begin{array}{l}\text { Mentor Graphics } \\
\text { "Mayflower" }\end{array}$ \\
\hline Wants to be in the race. Top 2 or 3 . & Wants to WIN. "Finishing 2nd is worst than last" \\
\hline $\begin{array}{l}\text { When asked, "How many employees work at } \\
\text { Tek?" the CEO answered, "About half of } \\
\text { them." } \\
\text { Tek refered to as, "The lazy "T". }\end{array}$ & $\begin{array}{l}\text { Story of employee who moved his } 50 \text { gallon } \\
\text { fish tank to the office lobby, "...because Ill see } \\
\text { them more often here." } \\
\text { Story of Charie complaining "Every night } \\
\text { when I go home the parking lot lights are off..." } \\
\text { Timers had been set to turn them off as } 3 \text { am. }\end{array}$ \\
\hline $\begin{array}{l}\text { Minimal negatjve feedback. } \\
\text { "We're family" }\end{array}$ & $\begin{array}{l}\text { Goal for minimal employee tumover. } \\
\text { Realize need to face up to performance } \\
\text { problems. }\end{array}$ \\
\hline \multirow[t]{2}{*}{$\begin{array}{l}\text { Loser projects fed for years. } \\
\text { Slow response to markets. }\end{array}$} & $\begin{array}{l}\text { Port project killed after } 3 \text { months. } \\
\text { "Exccute". Get it done NOW. }\end{array}$ \\
\hline & $\begin{array}{l}\text { MGC beats Tek to their own employees with } \\
\text { offer leuer during CASE buyout. }\end{array}$ \\
\hline \multicolumn{2}{|l|}{ Cramped workspaces, many partitions. } \\
\hline Terminal room. & A workstation in each engineer's office. \\
\hline No showers for employees. & $\begin{array}{l}\text { Showers from day } 1 \text {. } \\
\text { Jacuzzi \& weight rooms in building } \# 1 \text {. }\end{array}$ \\
\hline Employee profit sharing. & No profit sharing. \\
\hline \multicolumn{2}{|c|}{$\begin{array}{r}\text { Pandware } \\
\text { Software }\end{array}$} \\
\hline $\begin{array}{l}\text { Planning emphasized, presentations had many slides. } \\
\text { Decision based on politics rather than best technical } \\
\text { information. }\end{array}$ & $\begin{array}{l}\text { "Vision" important. No planning staff. } \\
\text { "Plans are always wrong, its better to be able } \\
\text { to tum on a dime." }\end{array}$ \\
\hline Tek CAE software nuns on 7 hardware platforms. & is only on Apollo platform. \\
\hline
\end{tabular}

Figure 54, Contrasts between the MGC and Tektronix cultures. 
The founders of MG reacted strongly to this aspect of Tek's culture. They felt that Tek was satisfied with a second or third place finish, that they were happy as a good "also ran". The founders of MG wanted to have their company win. "Winning" (a theme discussed below) meant having the best people working hard, pushing themselves to their peak. Intensity and hard work are attributes of the MGC culture that are obvious to a visitor. Several of the founders stories communicate the value placed on hard work. One is about how in the very early days (still nine founders in the two room rented office), Charlie Sorgie complained that when he went home at night the lights in the parking lot were turned off. He really disliked going to his car every night in the dark. Couldn't Tom or Gerry talk to the landlord about this? Well they did. It seems the lights were on timers and were set to turn off at 3:00 am.

There are other stories about hard work and the intensity of the environment. One might question whether these were just stories or whether they truly reflect the work environment. Observations indicated that there were many occasions in which the culture was that intense.

One manager told me, "we hired one fellow from Tektronix. He had the technical background we were looking for but he just never understood the commitment here. He put in 6 or 7 hours a day and then spent time reading the paper... He lasted less than a month." (later in the same interview) "The productivity of our engineers here at MGC is about ten times the industry average." ... "The industry average is something like 200 lines of code per engineer per week. That includes design, code generation, testing and debug. We average something like 2000 lines per week." (This manager had years of experience at IBM and General Motors.)

One Friday afternoon an impromptu department "mixer" was called. Someone bought beer, pop and munchies and 20 people sat on picnic tables between 5 and $6 \mathrm{pm}$ and talked in small groups. Then they gradually drifted back to their offices to "get a start on the weekend".

One interviewee was one of the system administrators who keep the 300 Apollo computers (nodes) on the company network functioning. When a problem arises on one of the nodes it can potentially bring down the entire network. This would mean 300 
engineers looking for the system administrator. When a problem does occur the administrator typically has 2 to 3 minutes to fix it or isolate that node from the network. He successfully dealt with 3 or 4 node problems during the 40 minutes we talked. The system administrators carry walkie talkie radios ( 2 channels) so they can more easily work in teams in finding and solving problems. During our interview, there were 17 phone calls and as many radio conversations. We answered several $\mathrm{E}$ mail messages that arrived during our talk. We made two trips outside the office in response to the phone calls. These became impromptu discussions with engineers about scheduling software updates, and the movement and reconfiguration of nodes and disk drives. The pace of activity made me think of juggling.

Intense? Yes. But the impression was not one of stress or being overwhelmed. The system administrators had a huge smile on his face the entire time as he talked on the walkie talkie while simultaneously answering an E-mail message and offering apologies for the constant interruptions. He seemed on top of everything and enjoying the pace of his work. Once I was certain he reached for the phone BEFORE it rang.

\section{It's a Race!!}

One event I witnessed would make a good "Intensity" story. In March 1988 Tektronix announced they were selling their CAE business to Mentor Graphics. For $\$ 5$ million Mentor Graphics obtained certain technologies and offered a transition package for customers with Tektronix CAE systems to migrate to Mentor Graphics tools. The fate of the 200 plus people employed in the Tektronix CAE division was left open. Mentor Graphics indicated it would not simply hire those people, but would consider them in filling existing openings which at the time numbered about 100 .

The Human Resources department of Mentor Graphics is one group that is heavily affected by acquisitions. Employees in an acquired firm have different pay scales, benefits packages, stock options, all of which need to be converted to Mentor Graphics' systems. As it became clear that Mentor Graphics would not directly hire all of the people Tektronix would lay off, the work involved in this "acquisition" was magnified - there would be interviews and a selection process to determine which of the Tektronix employees would receive offer letters.

Tektronix management had not yet decided which people they would relocate in other groups and which would face termination. The Mentor Graphics HR department decided that they would accelerate their interview and job offer process in order to place offer letters in the hands of Tektronix employees BEFORE 
Tektronix could reach their own people with a decision on whether they would be offered other positions to stay in the company. When representatives of Mentor Graphics HR group contacted their Tektronix counterparts for information to begin the interview process the Tektronix people said that the time schedule that Mentor Graphics was proposing was totally unrealistic.

In eight long days (including the weekend) the HR staff arranged and participated in interviews with over 100 people, assisting managers in evaluating those interviewed, prepared offer letters and called each person selected to let them know that Mentor Graphics wanted them as employees. The offer letters were delivered on a Wednesday and included a clause that if no response were received by Friday, the offer was void. Tektronix had not made their relocation decisions by Friday and Mentor Graphics succeeded in beating Tektronix to Tek's own people, capturing what they considered the cream of the people in Tektronix's CAE division.

This story portrays the intensity of activity at Mentor Graphics but it is not repeated there. It simply does not stand out as a big accomplishment in a company where people regularly push themselves in order to win. People "have a lot on their plate" and there is considerable pressure to meet deadlines. Work weeks of 60 or 70 hours are commonly sustained for long periods when conditions merit. One engineer moved his 50 gallon tropical fish tank from home to the lobby of his work building because "he can see his fish more often with them heren. People talk about "burn out" as a major problem.

One perspective on this intensity is that the rapid growth and technological change makes doing business like being in a race. People choose to be in a race. If you want to win the race, you have to push yourself and strive for your best, otherwise others will pass you by. Several people commented that Mentor Graphics was less intense during the interviews than, say, three years earlier. "The parking lot used to always be full at 6 pm. Now its mostly empty by $5: 30 .^{n}$

Eacilities Examples (Cultural Definition of "Cost" and "Competitive")

A difference in the cultural definitions of "cost" can be seen in how MGC and Tektronix allocated money for facilities. A common approach (one used at Tek) is to place a cluster of computers in a special "computer 
room" where many employees have access to the equipment. Because engineers spend only part of their time using a computer, and substantial amounts of time in meetings, phone conversations, etc., this hardware clustering approach allows time sharing of equipment and a smaller total number can be purchased. The cluster approach therefore reduces costs and the logic is that the company is therefore more competitive (i.e., Tean and mean'). Measures of 'computer utilization' indicate that this is a more 'cost effective' strategy then giving each person a computer which will sit idle a greater portion of the time. The workstations used at MGC can cost over $\$ 40,000$ each and there are hundreds in the Company.

Several practices at Mentor Graphics seem to be contrary to the achievement of this "least cost" business model. For example, from very early in the organization's life, the goal was for each engineer to have his or her own workstation. Senior managers at Mentor Graphics often made comments like, "If an engineer has a good idea, I don't want him to have to walk down the hall (to the computer room) before he can work on it. People and their ideas are the core of our business. If we cut corners to save a few dollars, we reduce our competitive edge and lose big dollars in the future." Here the model linking "cost" and "competitive position" make different assumptions about the "best" expenditures. The Mentor Graphics model suggests a greater valuation of employees. (A contrary view is that because MGC sold computers, they needed to show their customers a model where each engineer had a dedicated computer to support larger sales volume. The researcher discounted this view, as MGC management seemed sincere in their comments.)

Mentor also has a policy of providing each of its professional employees (or according to some, each of its 'engineers', a subset of 'professionals') with their own office - one with a door. In contrast at Tek, there is wide spread use of cubicles and partitions. Private offices represent a much higher "cost". Again MGC managers state that it is important that people have the ability to close the door when they so choose.

The availability of showers, weight room and sauna are another difference in facility expenditures between $M G$ and Tektronix. The first building MGC built had these because "it made a statement about the kind 
of people we wanted to hire and how they were to be treated." In contrast Tektronix resisted employee requests for shower facilities in the 1970s, 30 years after the company founding.

One conclusion consistent with these observations is that Mentor Graphics management placed a high value on employees and believed that a larger investment in them would be a good business decision. "High value" here is in comparison to Tektronics which had a reputation in the 1970 s as being a very good place to work, a place where employees were treated "like family".

\section{Winning}

The first value in the Mentor Graphics Corporate Values Statement is "Win, Ethically". The creation and ranking of the four values was a task to which the top 15 people in the company devoted a two day seminar. In addition, Dick Anderson, the project sponsor, indicated his feeling that "winning" is the core of Mentor Graphics culture. On a more empirical note, informants consistently pointed to achievement and 'making a contribution' in Q-sort exercises as primary to the Mentor Graphics culture. There can be little question that winning is a major aspect of the style of Mentor Graphics.

The term "Winning" implies accomplishing a challenging task, doing your best at a job, achieving recognition on some measure (e.g., Mentor Graphics was number one in worldwide sales of EDA systems in 1988 with 57\% of the market). Emplcyees often distinguished Mentor Graphics from Tektronix by pointing out that Mentor wants to win and puts in the extra effort required to do so. In contrast, Tek does just enough to keep in the race. The theme of "intensity" is closely related to the winning theme, though the definition of winning includes success and accomplishment. Intensity alone is not enough to be a winner at Mentor Graphics.

One general manager summed up the company's culture by questioning one of the value statements, "Challenge our employees" (Figure 57, discussed below). He said "challenge yourself is a more accurate statement of the culture. Set a high mark on the wall and go out and achieve it." In the cultural video, Brian Keirnan discusses how giving 
employees the freedom to succeed or fail causes them to set goals for themselves "far in excess of what any organization could place on them." Another described Winning as "reaching for the stars, like in the song, The Impossible Dream." In each case freedom, high standards, and accomplishment are included in the definition of Winning.

Another manager considered one goal for the Company was to be known for "being the best people managing company. We don't want to measure our success only with earnings. Mentor uses 'Winning' like other companies use the term 'excellence'."

Another said, "We want to develop self confidence like is found in the dynasties of the Lakers or Yankees. We want to earn an aura so that others say, 'golly, they are impressive'."

Winning also was related to leadership at Mentor Graphics by numerous employees. "Leaders are people who dream the dreams, and who communicate the dream. Others come to hear. We want dreamers, risk takers, skilled craftsman (dreamer hat first)."

\section{Locus of Control}

"The feeling of being in control of one's destiny" was often mentioned as important at Mentor Graphics. People repeatedly expressed confidence in their ability to accomplish difficult tasks and in the Company in overcoming large obstacles to succeed. For example on the day of the 1987 stock market crash one vice president said, "lets ignore those people (wall street) and just focus on running our business well. They'll come to their senses eventually." When the researcher asked one manager about the change from competing against Daisy to competing against IBM he said, "We can run rings around IBM." During a class on the new $\mathrm{C}++$ computer language it was pointed out that Bell Labs hadn't finished this language yet, leaving the status of some operations unknown "...but that's O.K. We can rewrite the compilers as necessary."

Several managers mentioned the need to give control to people in order

for them to be winners. The video tape segment on 'Coaching' presents the belief that managers often tend to "over control" thereby reducing the 
perception of control of the coached person. The advice in the tape is to resist the tendency to over control, give employees freedom and choice.

The belief that it is necessary to give employees freedom and a sense of self control was emphasized when a vice president mentioned watching a documentary on the death penalty to make a point about the importance of having control over one's destiny. He stated that "the death penalty is more humane than life without hope" (i.e., death is preferred to a life sentence, a situation of little control over one's destiny). At first this statement seemed extreme. But actually it is not too far from "give me liberty or give me death", an enduring value statement of one of the Country's founding fathers. It is indicative of a strong value of self determination.

The 1985 annual report described the Compute Engine development project. A quote from an engineer states, "my manager didn't stand over me while I designed the CPU." Another states, "Management supported my idea and gave me the responsibility." These are statements similar to those made during the interviews. Job opening announcements often state that employees are "driven from within, not from above". One interpretation that was offered of decision by Mentor Graphics to spin-off Context as a separate business was that this would give those employees the sense that they were making the decisions that determined their success or failure.

Researchers who have explored the 'locus of control' concept contrast those who have an 'external' and those with an 'internal' locus of control. The latter feel their actions influence their environment (in control of one's destiny) while those with an external orientation perceive that their fate is driven by forces beyond their control. Leftcourt (1982:80-81) concludes his review of the research noting that "Internals have been found to be more perceptive to and ready to learn about their surroundings. They are more inquisitive, they are more curious and efficient processors of information than are externals". He offers the logical explanation that "... a disbelief in the contingency between one's efforts and outcomes should preclude achievement striving. ... characteristics, essential to any prolonged achievement effort, will occur only among individuals who believe that they can, through their own efforts, accomplish desired goals. Individuals 
must entertain some hope that their efforts can be effective before they can make the sacrifices that are prerequisites for achievement." There is therefore some evidence to support the MGC belief that giving employees "a sense of control over their destiny" may lead to improved performance. The emphasis on a model where an internal locus of control produces greater employee productivity is certainly consistent with the theme of the Organizational Development literature. But companies such as Tektronix were considered leaders on this dimension. Perhaps Mentor Graphics represents a more extreme example of the application of that model.

\section{Eun}

Although there is considerable pressure to "execute" and get things done at Mentor Graphics there is also an emphasis on fun. Gary Larson's Far Side cartoons were in copious supply on doors, desks and windows throughout the company. A number of posters - like the one portraying specific MGC engineers as members of the Star Trek crew - were created by MGC employees from time to time to recognize some event. These were scattered along the halls of the Beaverton buildings. Mixers regularly involved costumes, special t-shirts, or sport - such as an egg toss, water pistol duels, volleyball competition and dance contests. In 1986 Tom Bruggere called a meeting of employees where the agenda was, "We seem to be having less fun. What can we do about this?"

\section{Bureaucracy, Growth and the "Small Company Atmosphere"}

There was considerable concern expressed during the interviews that Mentor Graphics' was becoming more bureaucratic as it grew. Many mentioned that the company was losing its "small company atmosphere". The definition of bureaucracy and the likelihood of becoming more bureaucratic are the central focus of the Quality Growth frame of reference in the simulation.

Most interviewees who addresses this topic seemed to be resigned to the belief that the company would become "more bureaucratic" as it grew larger. This resignation seemed an anomaly given the substantial evidence for the theme "we are in control of our destiny", which was so often 
expressed. People frequently expressed confidence in controlling such issues as technology change, competitive threats (such as IBM, HP), and global economic forces (i.e., stock market crash, exchange rates, protectionism). These were issues where it seemed that Mentor Graphics was a small cork in a big ocean. Yet they projected feelings of being able to maintain control over such situations. In contrast, they expressed the feeling that they had little control over changes which, if they occurred, would be entirely within the company - a change in their culture. (A contrasting view is that they understood these other issues well while they had little awareness of their own culture, making the latter seem less controllable. The intent of the simulation was to establish in internal locus of control for the domain of "organizational growth and culture change".)

The terms "bureaucracy", "big organization" and (to a lesser extent) "political organization" were used interchangeably by some of the people. These terms almost always carried a negative connotation, especially when used concerning Mentor Graphics. People at Mentor Graphics expressed the attitudes in Figure 55 about bureaucracy.

Bureaucracies were less desirable places to work

Organizations become more bureaucratic as they become larger

Bureaucracies were a result of increases in size / age in organizations

People in bureaucracies have little access to the leaders of the organization

People in bureaucracies have little control over their own success or failure

(they feel like "just another cog in a machine")

People in bureaucracies can have little or no impact on the organization

Bureaucracies are impersonal

Bureaucracies were inflexible and slow to change

Bureaucracies have rules and procedures which constrain people

Bureaucracies are organizations that tend to take few risks

People in Bureaucracies take few risks

In Bureaucracies, relationships were often "very political" in nature

People in bureaucracies tend not to know what is going on in other parts of the organization

Figure 55. Attributes of 'bureaucracy'.

In contrast, Mentor Graphics was described as having a "small company atmosphere" or a "start-up mentality" or an "entrepreneurial culture". These terms were used in a very positive manner and were contrasted with "bureaucracy" or "big organization". Companies with a 
small company atmosphere, and Mentor Graphics in particular, were described as having the characteristics in Figure 56.

SCAs are more fun, more exciting places to work than bureaucracies

In a company with a SCA there is rapid continuous change

People at Mentor Graphics have access to the leaders

In a organization with a SCA individuais can have an impact on the organization

Taking risks is part of a SCA

The feeling of 'I can make a difference' is a very important attribute of a SCA

In a SCA people have a vision of where the company is going and how they fit in

In a SCA, people know others throughout the organization

Figure 56. Attributes of a 'small company atmosphere' (SCA).

\section{"Pushing Back"}

The strongest request made by senior management for a change they would like the simulation to produce was to encourage more "pushing back". President Gerry Langeler comments in the video tape that "If you don't push back, we die", a statement indicating the importance to the Company of individuals making their voices heard and working to change things. In another segment of the video John Stedman notes, "how we did things doesn't hold weight around here and I hope it never does". Brian Kiernan also emphasizes the need to "question our systems and processes". A number of the stories that were told in the interviews were about examples where someone had pushed back. One employee told the researcher the measure of success for this study of the culture would be if it "tells us what's wrong $A N D$ how to fix it". This request for feedback and corrective measures exemplifies the value and expectation of pushing back. (The WADA simulation is the answer to his request.)

Pushing back was institutionalized in the annual MUG. Each day's presentations and workshops ended with all 200 to 300 customers seated in an auditorium. Perhaps 150 Mentor Graphics' employees stood around the room perimeter. There was an hour of 'open microphone' when customers asked questions and MGC employees who had responsibility on that topic came forward to explain, offer solutions and make commitments for improvements. Some of these were very painful confrontations. Customers complained bitterly about changes, omissions, and unmet promises. 
Employees did not indulge in 'finger pointing' or attempts to shift the blame. They said, "I'm sorry about that. I screwed up. Here is the work around which is the best we can offer."

These statements and stories were in contrast to responses on the questionnaires in 1990. There employees reported that pushing back was not a rewarded behavior (Figure 40). On one questionnaire an employee wrote in the margin, "pushing back is interpreted as trouble making".

\section{Risk Taking}

When asked what ideals the simulation should portray, the president immediately responded with "Risk Taking" and "Pushing Back". The "small company atmosphere" frame of reference designed for the simulation therefore includes these. Mentor Graphics' leaders encouraged risk taking with several activities. Stories about the "early days" refer to the first office as a "bookie joint" and describe many risks the founders took. Quitting their jobs to start a company, betting their success on Apollo who had yet to build a computer, demos at their first Design Automation Conference with software that was still crashing, are all portrayed as risks that paid off.

"New risks, new gains" was the sales force theme for 1988 and the Company's 7th birthday featured casino gambling in the cafeteria. Employees were issued play money and the payoff rules had been slightly modified so that, on average, the house had a net paid out. A year later, when an employee had an opportunity to take a risk (fly to meet with a customer) but did not act on it, the president wrote an article in the company newspaper stating that not going was a mistake. He pleaded that the company needs people to take risks.

\section{ADDITIONAL MGC CULTURE OBSERVATIONS}

\section{MGC Culture Supporting Activities}

Managers at Mentor Graphics engage in a number of activities to support the culture. In the fall of 1985, the MOC conducted a two day offsite that focused on the type of culture they wanted for the company. Figure 
57 displays the "Statement of Values" that was created during those discussions. In mid-1986 four posters and a 6 page employee booklet were createed to help convey those values to employees. Each employee received the booklet and copies of the posters appear in all company buildings.

The company newspaper,Voices, is published monthly and mailed to employees homes. It contains numerous articles written by company leaders. CEO Tom Bruggere writes a column for each issue that appears on the front page. These contributions to Voices help portray the culture and the ideals that are considered important. They provide an interpretation of events that helps define the reality of being a Mentor Graphics' employee. At the Company's 1987 birthday, "The Mentor Graphics Story", a 28 page booklet describing the company's early days, was distributed to all employees. It contains many vignettes about how problems were solved, the type of work and its quality, and relationships among people in the company's early days.

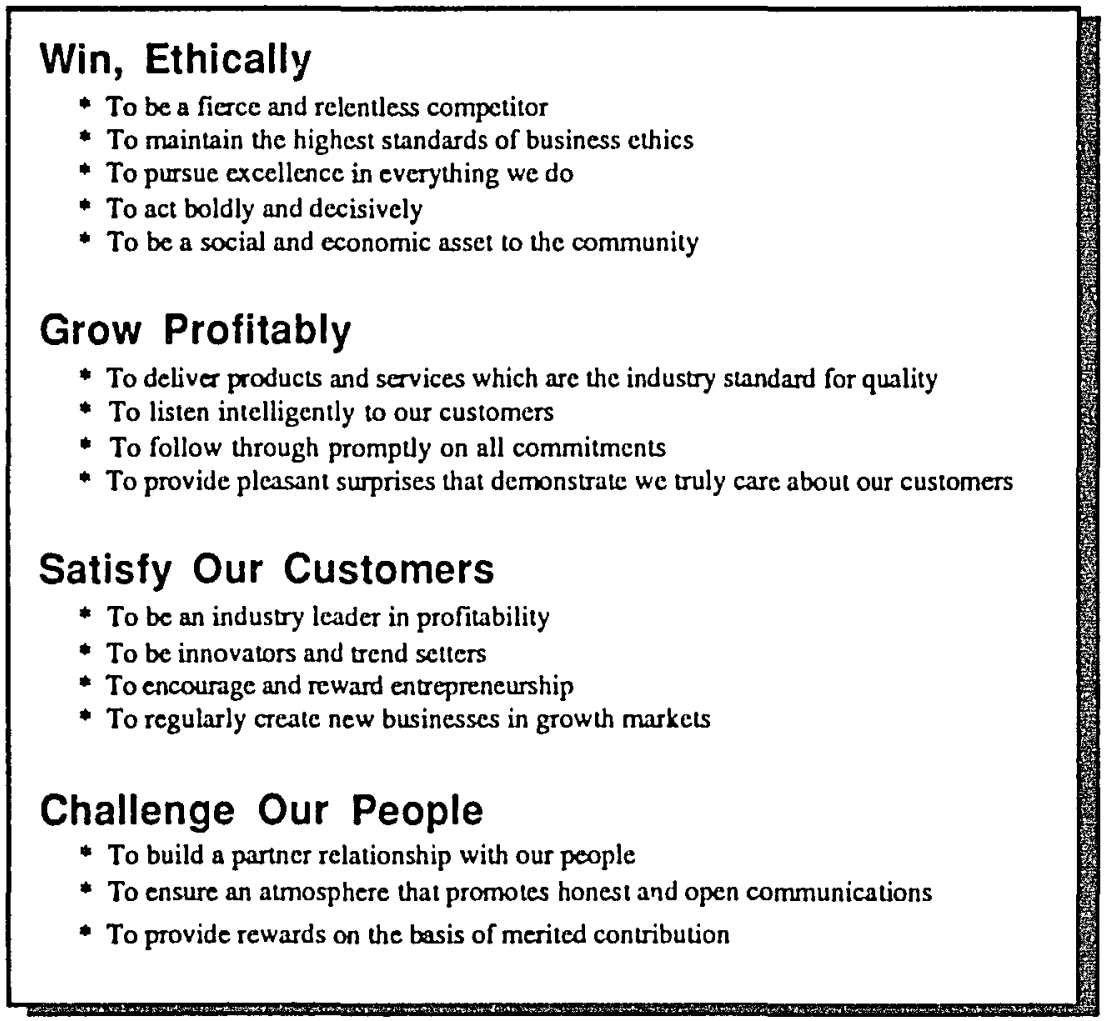

Figure 57. Mentor Graphics Statement of Values. 
Senior managers hold end-of-quarter meetings with all employees in the cafeteria. Similar meetings were held for any special events, such as an acquisition, new product shipment, etc. These meetings allowed employees to hear about events first hand and have an opportunity to ask questions about events that affected their company. During 1987 people began to comment more frequently that "the company is getting to big for all of us to fit in the cafeteria. How can we hold these meetings?" Later it was decided to hold two meetings and invite half of the employees to each. Still, there was a recognition that the means of conveying the culture to employees that worked well when there was 200 employees did not have the same effectiveness as the company approached 2000 employees.

\section{Anomalies Obseryed}

Numerous potential topics for the simulation emerged as the interviews progressed. Someone suggested that difference between the San Jose and Beaverton offices should be explored. A visit to San Jose uncovered no dramatic differences and the travel costs associated with more detailed analysis were a deterrent. Another obvious topic was the contrast between hardware and software engineers. This was described as a significant source of problems in the Compute Engine project. However the small numbers of hardware engineers after the break-up of the Compute Engine team made this topic less attractive to the Client. Providing an interpretation of the CE project in order to 'heal past wounds' and suggest changes in 'Project Management' that would avoid similar problems in the future was briefly considered.

Hiring practices were an obvious topic as a significant amount of managers time went here. In addition, when a company is growing that fast - the company doubled in size every 15 months - hiring decisions can be the single biggest determinant of the culture. However the feedback was that "the hiring process is working well, leave it alone". The definitions of Risk and Risk Taking, for individuals and the organization was another topic, one that was included as a small component in the final frames of reference.

The researcher's previous work in the utility industry sensitized him to cultural biases that hid strategic problems surrounding market 
saturation and changes in growth patterns. These issues were an obvious choice for the simulation topic but MGC cultural beliefs that the market had no foreseeable boundaries were very strong and topics of strategic interest may have encountered problems with client confidentiality.

The "hard work, long hours" topic was the most obvious choice but the researcher did not believe he could suggest greater efforts here and a research requirement for the simulation topic was to demonstrate change.

\section{Simulation Topics Selected}

In the fall of 1987 three topics were selected and approved by the Client's senior management. These are described in Figure 58. The essence of the "Quality Growth" frame of reference is to suggest a model in which employees realize that many choices that they make effect the culture of the organization. Therefore they can be "in control of our own (cultural) destiny".

\begin{tabular}{|c|c|c|}
\hline $\begin{array}{c}\text { Issue } \\
\text { Descritption }\end{array}$ & $\begin{array}{l}\text { Frame of } \\
\text { Reference }\end{array}$ & $\begin{array}{c}\text { Suggested } \\
\text { Changes }\end{array}$ \\
\hline $\begin{array}{l}\text { Trend toward bureaucracy, loss } \\
\text { of "small company atmosphere". } \\
\text { No one with Opportunity or } \\
\text { Responsibility to work against } \\
\text { adverse culture change. Passive } \\
\text { acceptance of change - External } \\
\text { Locus of Control. }\end{array}$ & $\begin{array}{l}\text { Quality } \\
\text { Growth }\end{array}$ & $\begin{array}{l}\text { Each individual has Opportunity \& } \\
\text { Responsibility to work against } \\
\text { adverse culture change. Instill } \\
\text { Internal Locus of Control - active } \\
\text { resistance to bureaucratic change. } \\
\text { Sum of efforts is sufficient to avoid } \\
\text { Bureaucarcy. }\end{array}$ \\
\hline $\begin{array}{l}\text { Job responsibilities growing } \\
\text { faster than people. Need for } \\
\text { more individuals to excell, } \\
\text { Definition of "Winning" unclear } \\
\text { in evolving market. }\end{array}$ & $\begin{array}{l}\text { Coaching } \\
\text { Winners }\end{array}$ & $\begin{array}{l}\text { Define "Winning" as high } \\
\text { achievement with Internal Locus of } \\
\text { Control. Spread coaching knowledge } \\
\text { of best MGC managers. Distinguish } \\
\text { manager vs leader roles. }\end{array}$ \\
\hline $\begin{array}{l}\text { Growing number \& diversity of } \\
\text { employees causes feeling of } \\
\text { alienation, loss of identity. } \\
\text { Vision becoming fragmented. } \\
\text { Need to give feedback without } \\
\text { conflict. }\end{array}$ & $\begin{array}{l}\text { Building } \\
\text { Partnerships }\end{array}$ & $\begin{array}{l}\text { Knowing others, understanding } \\
\text { plurality of visions at MGC reduces } \\
\text { alienation. Building partnerships with } \\
\text { others at MGC speeds flow of } \\
\text { information and creation of meaning, } \\
\text { supports vision and feedback. }\end{array}$ \\
\hline
\end{tabular}

Figure 58. Frames of Reference defined during the interviews. 
The "Coaching Winners" frame of reference was a response to numerous statements and requests made during the interviews that there was a need for more coaching of employees. People admitted that few in the company new how to coach or even had a clear understanding of what that meant. In a meeting of sales managers in May of 1987 a manager commented that there was a need for a discussion to help managers do a better job coaching. The session presenter offered an impromptu 10 minute presentation on coaching. While this was considered helpful, the feedback was that this was directed at "what to do when you have problem or marginal performers. We need help in coaching our star or near star people - people who are clearly not a problem, but who could really excel with coaching.

While it seemed that there was a need for more and better coaching, it was not initially obvious what would be the content of such a frame of reference for the simulation. One manager was referred to as a person who had expertise in coaching. Several interviews with that manager a a few others were the source for most of the "Coaching Winners" frame of reference. The basic model is that "the key to coaching is to give employees the feeling that they are in control of their own success or failure". One important step to achieve this feeling of control is to give employees complete control over their schedule. The belief is that, "The most demotivating thing for an employee is when a schedule they believe is unrealistic is delivered top down". This coaching model is consistent with the "internal locus of control" cultural theme. Yet is was not widely known in the Company. The goal in creating the "Coaching Winners" frame of reference was to diffuse the coaching model used by a few managers to others in the organization.

\section{MGC cultural drift from 1988 to 1990}

At the time the proposal was written, it was not anticipated that the culture of the organization selected for the study would undergo significant change during the course of the research. Within three months after the first interview, several employees had asked the researcher if he thought the culture of the Company was changing. The concern they expressed 
was about more "bureaucracy", which they indicated was inevitable. Utimately, this concern became the focus of the simulation.

The research project lasted considerably longer than originally expected and the researcher had the opportunity to observe the organization over a period of several years (Figure 48). A number of observations support the conclusion that the organization became more "bureaucratic" during the research project and that employees felt much less "in control of their own destiny" during Phase 4 of the project when the simulation was used and questionnaire data collected, than during the initial interviewing. (Figure 41 in Chapter V presents a small amount of questionnaire data supporting this claim. Measurement of such changes was not the focus of the questionnaire.) The events and interpretations of Mentor Graphics' 8.0 project was the biggest factor contributing to these changes. However there were also forces at work that had a more gradual influence.

An early and striking change the researcher noted regarded the posters created by employees that had been observed in the halls of the Beaverton buildings. (More than a dozen of these were photographed in 1987 as part of creating the ethnographic record.) A fellow graduate student was invited to visit MGC in the spring of 1988 to be given an informal tour by the researcher and to meet with a few employees. During this tour none of the posters could be found in the halls and only a small number were seen inside employees' offices. In asking about this change, the researcher was told, "...probably the corporate art committee had them removed...". This explanation was followed with the comment linked to most unwelcome changes, "...part of becoming a larger company...". The posters represented a very personal statement of involvement and commitment by employees. Their removal reduced the feeling of "small company atrnosphere" where individuals were identified for their contribution.

It was about this time that MGC moved into building 4 on the Beaverton campus. This building was taller and had an exterior that distinguished it from the other three buildings on the campus. All of the company's senior management were to have offices on the top floor. Several people commented about this move to "executive row", and how they 
expected they would have less access to senior management after the move. The company president acknowledged that there would be a "greater need for managers to go out to other buildings" with this change but the reality was that that effort was not made. Employees in some departments volunteered during interviews that they had not seen the half dozen most senior managers in those departments for years. This observation was always expressed with disappointment. Several of the top mangers never did relocate to "executive row" preferring to have their offices near the employees they managed. (When the Company moved to its Willsonville headquarters in 1990 the senior managers were again disbursed in the separate buildings where their departments were located.)

Prior to 1986 the company had thrown a "Pittock party" each August at a local landmark and this was a gala event. The economic and earnings outlook was poor in the summer of 1986, the company instituted a hiring freeze and even considered layoffs. It was announced that there would be no Pittock party that year, and there were none in future years. Many employees mentioned the loss of the Pittock party as a sign of a diminished small company atmosphere during the interviews. In 1988 there was no Company birthday party, an event that had always previously warranted a mixer. In 1991, a party was thrown at the Willsonville campus but few people showed up and one report indicated that senior management was unhappy with that outcome.

Many employees took the opportunity of the interview to express unhappiness about various changes in the Company to the researcher. (Though through 1988 all expressed overall satisfaction with their jobs.) Some commented on the loss of intensity as measured by the parking lot emptying early. Others said the company had "fewer mixers" and "Tom and Gerry don't mix" at those functions. (Unfortunately the researcher did not keep a detailed list of all mixers to provide an 'objective' measure of these reported changes.) The content of senior managers discussion of "vision" focused more on financial measures and less on how people would feel about working for the Company. "Becoming a Fortune 500 company" and "A billion dollars in revenue" were used to describe the vision of the 
future. But dozens of employees commented to the researcher, "Gerry hasn't said WHY he wants MG to become a billion dollar company."

At one mixer several employees suggested that the researcher get a job as "Mentor Graphics' ombudsman" after the study ended. At the time that comment was interpreted only as employees having fun with the student researcher. After all, with the Company's "open door policy" and senior management stressing the need to "push back" and "take risks", what need would there be for an ombudsman? But perhaps there were undercurrents of change that were becoming stronger.

Another indicator of gradual culture change is reflected in the effort to convey the culture to new employees. One employee who joined the company in 1986 proudly stated that during her first week of employment, one of the four founders had talked with her for 90 minutes, carefully explaining the vision of the company and the type of company they were trying to create. Other early employees made similar statements. In 1988 a 'new employee folder' had been created but the person teaching the new employee orientation class confided that, while he was comfortable explaining the benefits and related programs, "... when I get to the subject of vision and culture I really feel lost. I show them the corporate values statement, but don't have much more to offer." The founders rarely met with new employees.

Leaders in some other companies have continued to make greater efforts to contact new employees. The CEO of Sequent Computer, for example, participates in every new employee orientation class. It can be concluded that significantly greater effort to share the vision and culture is possible. It simply was not a priority at MGC after 1987.

During the video tape production and simulation programing (Phase 3 ) the researcher was employed teaching in Corvallis. Simulation topic selection and frame of reference design had been completed and data from the pilot questionnaire confirmed these as valid targets for change. There was considerably less contact with the members of the culture during this time. As the simulation neared completion and classes were scheduled for November of 1989, there was again more contact. This coincided with the "8.0 announcement" and the changes associated with that project. The 
researcher was surprised to find a number of people unhappy with the organization and the related changes. Prior to this time many had expressed "we need to change $\mathrm{x} . .$. " but there was always a positive outlook, an internal locus of control associated with those comments. Now some people were genuinely unhappy.

Several expressed that they or others they knew were sending out resumes because of how they were being treated. One employee said, "I'm just at the bottom of the pile, I can't do much to change things". This was a long way from "in control of my own destiny" and struck the researcher as being very out of character for anyone at Mentor Graphics. Comments written on the questionnaires in 1990 reflected these changes. Although only about $20 \%$ of the questionnaires contained any written remarks, nearly all of these were negative. For example one engineer wrote, "I feel upper management doesn't have the slightest idea of what's going on in engineering for 8.0. This is the most stressful, intense, unfeeling place I've ever worked. All that bullshit in the Annual Report caused me to lose all faith in Tom and Gerry's credibility. THIS IS NOT FUN."

A number of post-simulation interviews were conducted to collect feedback from participants. One vice president acknowledged that Mentor Graphics “...fell into trap of managing, instead of leading, during the last two years" ( 8.0 project). Another vice president summarized "the one important lesson" from the simulation, "We stopped investing in culture and people in about 1986 and have been coasting since then. The simulation shows that you can only coast so long and then there is pain."

The culture of Mentor Graphics always valued "long hours". This was a key to winning in their world view, and one point where they wanted to distinguish themselves from Tektronix. When people choose to put in long hours they can still feel "in control of their destiny" despite a considerable workload. It seems that during the 8.0 project the leaders forgot the importance of individual choice and focused only on "long hours".

When employees were told they must cancel vacations, work weekends, forgo all training, accept job transfers without the individual's input, the Company does indicate "long hours" are important. But without choice for the individuals involved, "people are driven from above and not 
from within", the opposite of what the Company claimed it wanted. Individuals who were transferred during the 8.0 project were referred to as "draftees" a term that conveys the absence of an internal locus of control and a mindset of "just following orders ...just doing what I'm told" which is the antithesis of what this researcher identified as the core of the MGC culture, "challenge yourself". The attitudes and interpretations that developed were not supportive of the exceptional individual contributions that management wanted and the Company needed.

\section{Climate During Simulation Delivery}

There were high levels of stress among some of the people at Mentor Graphics during the 8.0 project which began about a year before the August simulation classes. Some engineering groups had considerable stress while other groups were relatively unaffected. The project had missed some deadlines that the Company had set for itself. In January a senior manager in engineering with more than 5 years at the Company was fired "for cultural reasons". "Although he achieved results, he didn't create the culture we wanted." In late July three high level engineering managers were given new jobs with no management responsibilities. One immediately resigned. Coincidentally, Iraq invaded Kuwait the week before the first simulation class.

The welcome letter sent to all participants in the August session of classes indicated that there was a need to "revisit our values now that we're 10 years old". One of the Organization's stated purposes for the classes was to gather input from the participants about the culture. A lot of opinions were expressed during those six classes and that feedback was presented to senior management during the following week. Soon after that it was announced that senior management would conduct a series of "focus groups" with employees to learn more about problems and formulate responses. The welcome letter letter for the October session included a statement that one purpose was to determine, "What's wrong and how do we fix it?" Shortly after the final Control group post-simulation questionnaires were received, a new position of vice president of Employee Relations was created that reported to the CEO. 


\section{References of Special Interest}

During the project, the researcher became aware of several written works that were helpful in understanding the culture of Mentor Graphics. The first of these was The Soul of a New Machine (Kidder 1981). A general manager recommended this in an early interview suggesting that it would be useful for understanding the Mentor Graphics culture. Later when this recommendation was mentioned to another manager, the response was different. "The intensity is similar, but the Kidder story describes a situation where there was a lack of trust, people were motivated by fear. That is not an accurate description of MGC. This is a much more open, supportive environment."

A second book is The Macintosh Way (Kawisaki 1990). The author provides an entertaining look at the culture of Apple Computer in its early days. The ideals that he argues are so important are very similar to those at MGC. Odyssey (Sculley 1987) also provides some understanding of the Apple culture though a greater volume of reading is required for comparatively little insight. The "Third Wave" corporation model (p 92-100) is a succinct statement of the general transition in management philosophy now underway in U.S. businesses. Mentor Graphics is on the leading edge of that transition.

Block (1987) describes "bureaucratic" and "entrepreneurial" cycles in organizations and this contrast is similar to the "bureaucracy" and "small company atmosphere" frames of reference portrayed in the WADA simulation.

Finally, the descriptions of "manager" and "leader" offered by Zalesnik (1977) were extremely useful in defining the model of coaching in the "Coaching Winners" frame of reference. 
APPENDIX D

FINAL QUESTIONNAIRE 
To: $\quad$ Selected employees of Mentor Graphics

From: Tom Bruggere

Subject: $\quad$ Attached Corporate Culture Questionnaire 0000000000000000000000000000000000000000000000000

As many of you are aware, Terry Schumacher has been studying Mentor Graphics to collect data for his dissertation on corporate culture. We agreed to this study not only to assist Terry, but because we feel the final output will benefit Mentor Graphics as well.

Terry has completed more than 200 interviews and has developed the attached questionnaire from the opinions expressed in those interviews. As part of our commitment to him, we agreed that our people would complete that questionnaire.

The quality of his final product and its potential value to the company is dependent on a high response rate. I have completed a copy of the questionnaire and can tell you that it was a painless task. I would ask that you also take the time to complete and return the questionnaire. Thank you. 
To:

From:

Terry Schumacher

Subject:

Attached Corporate Culture Questionnaire

The attached questionnaire was created from a large number of interviews with people at Mentor Graphics. There are many different viewpoints that together make up the Mentor Graphics culture. In order for me to learn how widespread within the company some of these viewpoints are, I am asking each of you to complete the questionnaire and return it to $\mathrm{me}$ in the attached stamped envelope.

Some of you may have completed an earlier questionnaire for me. I am happy to report that there was an $86 \%$ response rate. This is an outstanding rate in the questionnaire business. I regret that I have been unable to share with you the results of that questionnaire. The concem is that feedback about my findings would influence future responses. As I near the end of this study I look forward to making the conclusions available to each of you. If you would like to make any additional comments about Mentor Graphics I remain interested in taking with you. My home phone is 775-9378. My schedule is very flexible.

In completing this questionnaire, please consider:

Your responses will be kept completely confidential. The questionnaires and data are my property and not that of Mentor Graphics. Others at Mentor Graphics will not have access to the completed questionnaires. No one other than myself will see the raw data. Any reporting of the findings will be done in such a manner that it is not possible to identify an individual.

My analysis will be weakened by a low retum rate of the questionnaire or by partially completed questionnaires. Please complete the entire questionnaire by circling one answer for each item.

Please read each item carefully. Some items are different from others by only one or a few words.

There are no right or wrong answers for any of these questions. The "correct" answer is the one that most accurately represents your honest opinion on each issue.

The questionnaires are numbered so that I can send a follow-up reminder notice to anyone who does not return the questionnaire by the response deadline.

Please mail the completed questionnaire to me in the attached, stamped envelope. PLEASE MAIL THEM BY MAY 30th. I will send out a reminder notice to those people who have not returned their questionnaires by that time. Thank you. 
Select the one response for cach item that best matches your personal opinion about that item.

Strongly agree Moderatly agree

Slightly agree

Neither agree nor disagree

\section{Slightly disagre \\ Moderatly disagree \\ Strongly disagree}

1. I prefer working in an organization with a 'small company atmosphere' to working in a bureaucratic organization.

2. Mentor Graphics rewards managers who do a good job of coaching.

3. The biggest reward that a manager can give to employees is the sense of achievement that comes with completing a challenging task.

4. Mentor Graphics rewards individuals who take risks.

5. For the most part, upper management at Mentor Graphics does not use the "management by walking around" philosophy.

6. There are considerable opportunities for each employee to reduce or inhibit bureaucracy at Mentor Graphics.

7. It is necessary to have a feeling that one has control over the success or failure of a task in order to fecl like a winner in completing that task.

8. Mentor Graphics is the best company I've ever worked for.

9. A 'small company atmosphere' is nearly the opposite of a bureaucratic culure.

10. When individuals make the effort to express their opinions it helps the company avoid bureaucratic growth.

11. At Mentor Graphics the top management is available and willing to listen to the ideas of any of the employees.

12. One of the characteristics of bureaucratic companies is that employees in those companies take very few risks.

13. Mentor Graphics has been struggling during the past year as it works to produce the 8.0 project.

14. Managers at Mentor Graphics need to do a better job of communicating to employees how the employee's tasks fit into a vision of the company's future.

15. At Mentor Graphics there is a long term vision of where the company is going.
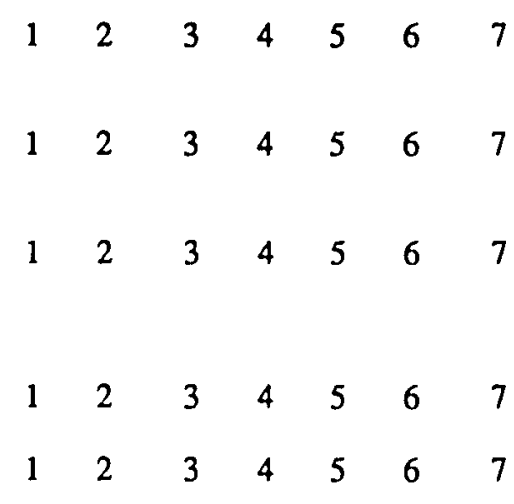

$\begin{array}{lllllll}1 & 2 & 3 & 4 & 5 & 6 & 7\end{array}$

$\begin{array}{lllllll}1 & 2 & 3 & 4 & 5 & 6 & 7\end{array}$

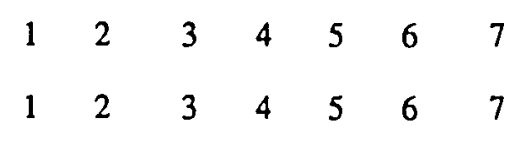

$\begin{array}{lllllll}1 & 2 & 3 & 4 & 5 & 6 & 7\end{array}$

$\begin{array}{lllllll}1 & 2 & 3 & 4 & 5 & 6 & 7\end{array}$

$\begin{array}{lllllll}1 & 2 & 3 & 4 & 5 & 6 & 7\end{array}$

$\begin{array}{lllllll}1 & 2 & 3 & 4 & 5 & 6 & 7\end{array}$

$\begin{array}{lllllll}1 & 2 & 3 & 4 & 5 & 6 & 7\end{array}$

$\begin{array}{lllllll}1 & 2 & 3 & 4 & 5 & 6 & 7\end{array}$ 
Select the one response for each item that best matches your personal opinion about that item.

Strongly agree

Moderatly agree

Slightly agree

Neither agree nor disagre

\section{Slightly disagre \\ Moderatly disagree \\ Strongly disagree}

16. A person gets the feeling of being a winner from achieving a goal which challenged that person's abilities.

17. The actions of managers at Mentor Graphics are frequently driven by short term pressures, such as the next release or next quarter's financial performance, to the detriment of longer term corporate goals.

18. The key to coaching is to give employees a sense that they are in control of their own destiny.

19. I often hear employees talk about some of Mentor Graphic's recent success stories.

20. As a company grows larger, building partnerships with others in the company becomes more important.

21. It is possible for Mentor Graphics to become very large while still retaining its 'small company atmosphere'.

22. The essence of a partnership is for each person to respect the viewpoint of the other.

23. Mentor Graphics under-invests in training for employees.

24. There is a greater effort put into building partnerships at Mentor Graphics today than there was two years ago.

25. Most of the people at Mentor Graphics expect that their job responsibilities will change completely over the next 2 to 3 years.

26. A major factor that can lead to an organization becoming bureaucratic is when the people don't bother to work against the growth of bureaucracy.

27. The shared vision in the department where I work is as strong or stronger now than it was two years ago.

28. In the future we will need to do a better job of forming partnerships at Mentor Graphics.

29. In order to give employees the feeling of being in control, the employees should be the only ones to change their schedule.

7

$\begin{array}{lllllll}1 & 2 & 3 & 4 & 5 & 6 & 7\end{array}$

$\begin{array}{lllllll}1 & 2 & 3 & 4 & 5 & 6 & 7\end{array}$

$\begin{array}{lllllll}1 & 2 & 3 & 4 & 5 & 6 & 7\end{array}$

$\begin{array}{lllllll}1 & 2 & 3 & 4 & 5 & 6 & 7\end{array}$ 
Select the one response for each item that best matches your personal opinion about that item.

Strongly agree

Moderatly agree

Slightly agree

Neither agree nor disagree

Slightly disagree

Moderatly disagree

Strongly disagree

30. As Mentor Graphics becomes a larger organization it will become more bureaucratic.

31. Mentor Graphics is willing to make costly investments in the short term in order to win in the long term.

32. I believe that I am expected to "push back" and make my opinion known when I hold a strong opinion that is in contrast to that of the majority.

33. People in a bureaucracy often have the feeling that they are just another 'cog in the machine'.

34. To maintain our the small company atmosphere at Mentor Graphics, we will need to put greater efforts into 'pushing back' in the future.

35. As organizations grow it is inevitable that they become more bureaucratic.

36. People feel like they have less control over their destiny when they have no vision of how they fit into the future.

37. Being a coach is one role that managers at Mentor Graphics are expected to play.

38. There are many times that form or appearance is given a higher priority than function at Mentor Graphics.

39. Managers have a special responsibility in achieving quality growth through their coaching of other employees.

40. We sometimes show litule patience with customers who are not as technically sophisticated as we are.

41. Without a great deal of diversity in the company Mentor Graphics would be unable to accommodate growth.

42. Learning what is going on in other parts of Mentor Graphics has litule to do with achieving quality growth in the company.

43. It is very expensive to go to the market with a new product and then have to do major rework to get it right.

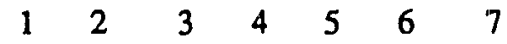

$\begin{array}{lllllll}1 & 2 & 3 & 4 & 5 & 6 & 7\end{array}$

$\begin{array}{lllllll}1 & 2 & 3 & 4 & 5 & 6 & 7\end{array}$

$\begin{array}{lllllll}1 & 2 & 3 & 4 & 5 & 6 & 7\end{array}$

$\begin{array}{lllllll}1 & 2 & 3 & 4 & 5 & 6 & 7\end{array}$

$\begin{array}{lllllll}1 & 2 & 3 & 4 & 5 & 6 & 7\end{array}$

$\begin{array}{lllllll}1 & 2 & 3 & 4 & 5 & 6 & 7\end{array}$

$\begin{array}{lllllll}1 & 2 & 3 & 4 & 5 & 6 & 7\end{array}$

$\begin{array}{lllllll}1 & 2 & 3 & 4 & 5 & 6 & 7\end{array}$

$\begin{array}{lllllll}1 & 2 & 3 & 4 & 5 & 6 & 7\end{array}$

$\begin{array}{lllllll}1 & 2 & 3 & 4 & 5 & 6 & 7\end{array}$

$\begin{array}{lllllll}1 & 2 & 3 & 4 & 5 & 6 & 7\end{array}$

$\begin{array}{lllllll}1 & 2 & 3 & 4 & 5 & 6 & 7\end{array}$

$\begin{array}{lllllll}1 & 2 & 3 & 4 & 5 & 6 & 7\end{array}$ 
Select the one response for each item that best matches your personal opinion about that item.

Slightly disagree

\section{Strongly disagree}

Moderatly disagree

44. The primary benefit of a partnership is enhanced communication.

45. There is liule that most individuals can do to prevent the spread of bureaucracy as an organization becomes large.

46. At Mentor Graphics, decisions are usually pushed down to the lowest appropriate level.

47. When managers at Mentor Graphics coach employees it helps the company to achieve quality growth.

48. Mentor Graphics employees have a responsibility to prevent bureaucracy in the company.

49. People at Mentor Graphics work more closely as a team than do people in most other high tech companies.

50. When the growth in an organization has sufficient quality, the organization will not become a bureaucracy.

51. An important goal of coaching is to help the coached person feel like a winner.

52. The term "partnership" is sometimes used at Mentor Graphics to refer to relationships between people within the company.

53. If the business environment were poor, Mentor Graphics would do almost anything rather than lay off people.

54. Each of the departments in Mentor Graphics makes a vital contribution to the companies success.

55. An organization fecls more bureaucratic to individuals who do not have a vision of how they fit into the future of that organization.

56. It is important to take the time to build partnerships with others at Mentor Graphics.

57. There are opportunities for each individual at Mentor Graphics to make certain that the growth in the company is quality growth.
Strongly agree
atly agree

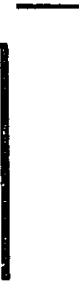

$\begin{array}{lllllll}1 & 2 & 3 & 4 & 5 & 6 & 7\end{array}$

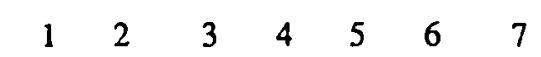

$\begin{array}{lllllll}1 & 2 & 3 & 4 & 5 & 6 & 7\end{array}$

$\begin{array}{lllllll}1 & 2 & 3 & 4 & 5 & 6 & 7\end{array}$

$\begin{array}{lllllll}1 & 2 & 3 & 4 & 5 & 6 & 7\end{array}$

$\begin{array}{lllllll}1 & 2 & 3 & 4 & 5 & 6 & 7\end{array}$

$\begin{array}{lllllll}1 & 2 & 3 & 4 & 5 & 6 & 7\end{array}$

$\begin{array}{lllllll}1 & 2 & 3 & 4 & 5 & 6 & 7\end{array}$

$\begin{array}{lllllll}1 & 2 & 3 & 4 & 5 & 6 & 7\end{array}$

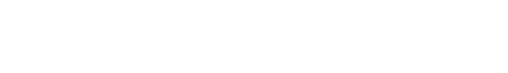

$\begin{array}{lllllll}1 & 2 & 3 & 4 & 5 & 6 & 7\end{array}$

$\begin{array}{lllllll}1 & 2 & 3 & 4 & 5 & 6 & 7\end{array}$

$\begin{array}{lllllll}1 & 2 & 3 & 4 & 5 & 6 & 7\end{array}$

$\begin{array}{lllllll}1 & 2 & 3 & 4 & 5 & 6 & 7\end{array}$

$\begin{array}{lllllll}1 & 2 & 3 & 4 & 5 & 6 & 7\end{array}$ 
Select the one response for each item that best matches your personal opinion about that item.

Strongly agree

Moderatly agree

Slightly agree

Neither agree nor disagre

Slightly disagree

Moderatly disagree

\section{Strongly disagree}

58. Each employee at Mentor Graphics has opportunities to 'push back'.

59. Helping others at Mentor Graphics see how they are having an impact on the company will reduce the feeling of being in a bureaucracy.

60. Employees who feel they have control of their own destiny are more likely to be top performers.

61. People at Mentor Graphics are rewarded for making the effort to see the viewpoint of others.

62. When an organization becomes burcaucratic, this almost always implies the loss of the small company atmosphere.

63. If each person at Mentor Graphics takes more risks, the company will feel less like a burcaucracy.

64. Employees at Mentor Graphics can help reduce bureaucracy by forming partnerships with people in other parts of the company.

65. Each member in a partnership has a responsibility to express their viewpoint to the other members of the partnership.

66. When an employee is forced to accept a schedule that is not of their choice, they feel less in control of their own destiny.

67. During the last 2 years, Mentor Graphics has lost some of its small company atmosphere.

68. At Mentor Graphics, people who 'push back' are rewarded.

69. One important part of coaching is to convey to the coached person how their tasks fit into the vision of the company's future.

70. When a person is successful in 'pushing back' and can see the impact they have had on the organization, they feel more in control of their own destiny.

71. As Mentor Graphics becomes a larger organization it will lose its small company atmosphere.

$\begin{array}{lllllll}1 & 2 & 3 & 4 & 5 & 6 & 7 \\ 1 & 2 & 3 & 4 & 5 & 6 & 7 \\ 1 & 2 & 3 & 4 & 5 & 6 & 7 \\ 1 & 2 & 3 & 4 & 5 & 6 & 7 \\ 1 & 2 & 3 & 4 & 5 & 6 & 7 \\ 1 & 2 & 3 & 4 & 5 & 6 & 7 \\ 1 & 2 & 3 & 4 & 5 & 6 & 7\end{array}$

$\begin{array}{lllllll}1 & 2 & 3 & 4 & 5 & 6 & 7 \\ 1 & 2 & 3 & 4 & 5 & 6 & 7 \\ 1 & 2 & 3 & 4 & 5 & 6 & 7 \\ 1 & 2 & 3 & 4 & 5 & 6 & 7 \\ 1 & 2 & 3 & 4 & 5 & 6 & 7\end{array}$

$\begin{array}{lllllll}1 & 2 & 3 & 4 & 5 & 6 & 7\end{array}$

$\begin{array}{lllllll}1 & 2 & 3 & 4 & 5 & 6 & 7\end{array}$ 
Select the one response for each item that best matches your personal opinion about that item.
Strongly agree

Moderatly agree

Slightly agree

Neither agree nor disagree

Slightly disagree

Moderatly disagree

\section{Strongly disagree}

72. When managers communicate the vision of the company to employees, they help prevent bureaucracy.

73. There is a need for individuals at Mentor Graphics to take more risks if we wish to ensure that the company's growth will be quality growth.

74. On average, the people in the group where I work feel more in control of their own destiny today than they did two years ago. 75. For Mentor Graphics to continue to be a leader in Design Automation we need to improve our performance in coaching our people.

76. The successful completion of 8.0 is THE big challenge facing Mentor Graphics today.

77. Compared to other companies of similar age and size, Mentor Graphics has a minimal amount of bureaucracy.

78. When employees push back they can inhibit the growth of bureaucracy at Mentor Graphics

79. When I am reading an interesting story or novel, I imagine how I would feel if the events in the story were happening to me.

80. I really get involved with the feclings of the characters in a novel.

81. I am usually objective when I watch a movic or play, and I don't often get completely caught up in it.

82. After seeing a play or movie, I have felt as though I were one of the characters.

83. I daydream and fantasize, with some regularity, about things that might happen to me.

84. Becoming extremely involved in a good book or movie is somewhat rare for me.

85. When I watch a good movie, I can very easily put myself in the place of a leading character
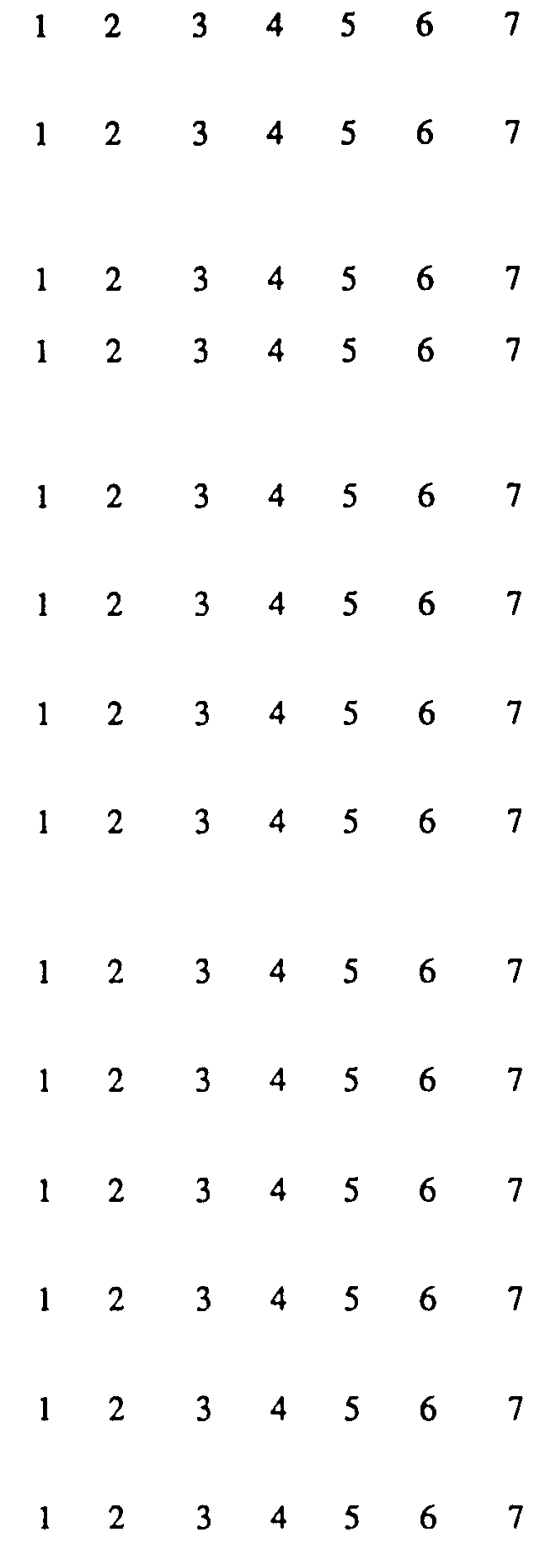
Select the one response for each item that best matches your personal opinion about that item.

\section{Slightly disagree \\ Moderatly disagree \\ Strongly disagree}

86. Before criticizing somebody, I try to imagine how I would feel if $I$ were in their place.

87. If I'm sure I'm right about something, I don't waste much time listening to other peoples arguments.

88. I sometimes try to understand my friends better by imagining how things look from their perspective.

80. I believe that there are two sides to every question and try to look at them both.

90. I sometimes find it difficult to see things from the "other guy's" point of view.

91. I try to look at everybody's side of a disagreement before I make a decision.

92. When I'm upset at someone, I usually try to "put myself in his shoes" for a while.

93. Today the biggest threat to the success of Mentor Graphics is the problems that result from growth within the company.

94. There is friction between various groups at Mentor Graphics that substantially reduces our corporate effectiveness.

\section{$\begin{array}{lllllll}1 & 2 & 3 & 4 & 5 & 6 & 7\end{array}$}

$\begin{array}{lllllll}1 & 2 & 3 & 4 & 5 & 6 & 7\end{array}$

$\begin{array}{lllllll}1 & 2 & 3 & 4 & 5 & 6 & 7\end{array}$

$\begin{array}{lllllll}1 & 2 & 3 & 4 & 5 & 6 & 7\end{array}$

$\begin{array}{lllllll}1 & 2 & 3 & 4 & 5 & 6 & 7\end{array}$

$\begin{array}{lllllll}1 & 2 & 3 & 4 & 5 & 6 & 7\end{array}$

$\begin{array}{lllllll}1 & 2 & 3 & 4 & 5 & 6 & 7\end{array}$

$\begin{array}{lllllll}1 & 2 & 3 & 4 & 5 & 6 & 7\end{array}$

$\begin{array}{lllllll}1 & 2 & 3 & 4 & 5 & 6 & 7\end{array}$ 
All questionnaire responses will be kept confidential. Any reporting of the findings will be done in such a manner that it will not be possible to identify an individual.

\section{Demographic information}

1. Sex female male

2. Education:

Never attended college

less than 2 years of college completed

$\geq 2$ but $<4$ years of college completed $\geq 4$ but $<6$ years of college completed more than 6 years of college completed

3. Length of time employed by Mentor Graphics

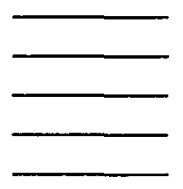

4. Comments: 


\section{APPENDIX E}

\section{WINNING AT DESIGN AUTOMATION RULEBOOK}




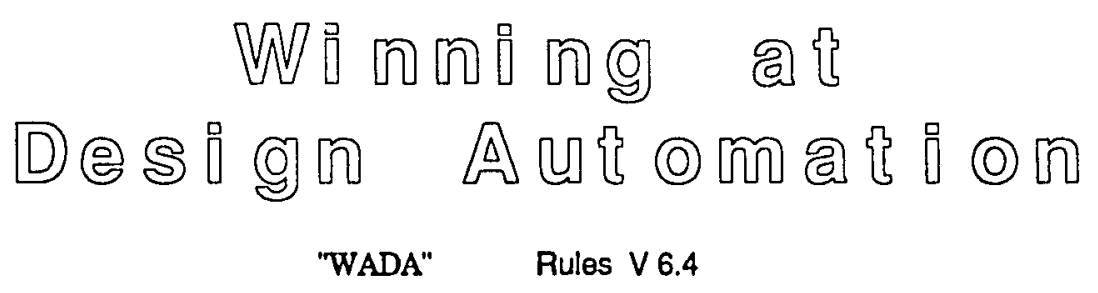

\section{Overview}

"Winning at Design Automation" (WADA) is a business simulation that runs on a Macintosh computer. Players receive two primary resources, Time and Personal Energy, and make choices on how to spend these resources to complete tasks. Some tasks require players to have a third resource, Information, and players spend some of their Time and Personal Energy to acquire Information. Players earn points for completing tasks and the goal to is accumulate the most points over the 60 to 90 minutes it takes to play the simulation.

"Winning at Design Automation" is designed so that players can focus their attention on some of the internal dynamics of organizational life during rapid growth in a high technology company. One facet of rapid growth that is of interest to many people in such a company is the effect of growth on the culture of their organization. "Winning at Design Automation" allows players to develop a broader understanding of the relationship between culture and growth by presenting players with two perspectives on culture. These perspectives are developed by playing the simulation twice and adopting a different role for each play.

In addition to the rules that describe the operation of the game, players are given a description of a culture and adopt that culture as a role to be played. In the initial game, players are "visiting" the Alpha company. They experience the growth in the Alpha company and become aware of how persons in the Alpha culture would respond to those events. The typical game experience is to play the Alpha culture for eight quarters, then discuss the results and observations. In the discussion at the end of the Alpha play of the game, players share their insights and opinions. They often describe differences and similarities between the Alpha culture and the culture of the company in which they work. Those contrasts provide further insights for the players.

In the second play of the game, players "visit" the Beta company and adopt the role presented in the description of the Beta culture. The Beta company is also experiencing rapid growth and players develop an understanding of how the managers of Beta respond to those events. After the game players compare their experiences from each culture.

The Alpha and Beta cultures are purely fictitious and the perspective that players develop is not how good or bad these cultures may seem, but rather an appreciation for those aspects of these cultures which have bearing on the cultures of their organization. 


\section{Playing 'Winning at Design Automation"}

\section{Setting and Goals:}

Each player is a manager of one department in Alpha, a hypothetical company. Groups of four managers play as a team who operate a division of that company. Each team is in competition with the other teams. To win, your team must grow your company to be the world leader in design automation. This is done by accumulating the most task completion points.

Each player makes decisions at his own workstation (Macintosh). Points are awarded to each player/department but it is the total points of each team that will be compared against other teams at the end of the game.

\section{Role:}

You are asked to consider the experience you will have as a manager in the Alpha company as an opportunity to see what it would be like to leave the company where you work for a short while and work in the Alpha company. You may consider this like traveling to a foreign country to see how the natives live. Although there are likely to be some similarities between Alpha and your company there will be differences as well and it is important for you to adopt the role of being an "Alphan" to fully appreciate both the similarities and differences. To help you understand the work life in the Alpha company, a description of the Alpha culture has been prepared for you.

After you have read the rules of the game, your team will be given some time to examine the description of the Alpha culture and to discuss it among yourselves. A detailed understanding of the Alpha culture will be of benefit to you while playing the game. (see "Get Info" card description on page 12.)

The culture descriptions give some guidance to players for making resource allocations. When the players adopt the cultural role for the short time they are "visiting" Alpha, it builds a common frame of reference which is the basis for discussion in the post-simulation discussion. Players then can better define how their organization is similar to and different from the fictitious Alpha and Beta cultures. You are encouraged to note your observations about the cultural roles in the space provided at the back of this booklet.

\section{3. "Blenders"'}

Players on a team work independently, each making decisions for their departments on their Macintosh. Teams may confer at the end of every few quarters in "blenders", a short time set aside for social interaction. Typically blenders last about 5 minutes. Often players will use this time to exchange what they have learned in order to help others on their team. The game facilitator will give you specific instructions about the frequency and time limits of your blenders. 


\section{Departments:}

Each manager plays one of the 4 departments that make up a company. The departments are;

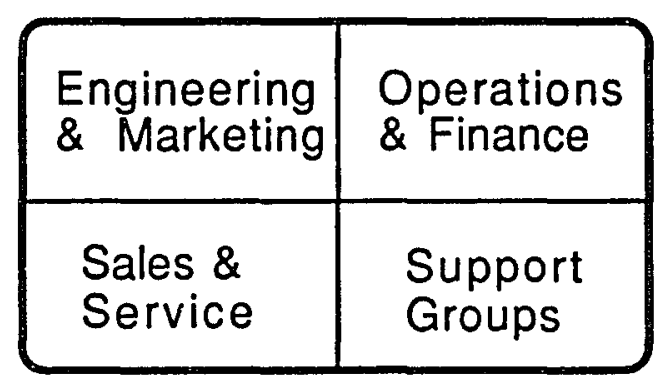

Figure 1. The four departments.

(Note: Support Groups includes Legal, Human Resources, Public Relations, and Facilities functions.)

Before the simulation begins, the four managers on each team will meet to decide on a team name and to determine which manager will play which department. There is no requirement of specific technical knowledge to play any of the departments. Players often select a department with a function that is different than their normal job.

\section{Resources:}

Time

The primary resource that managers receive each quarter is their time, and the time of their individual contributors. (These are considered as a single resource in the game, the distinction between a manager as an individual and representing a department being somewhat blurred.) Nearly all of the tasks and activities of managers in the Alpha company require some amount of time resource.

The amount of Time resource available is primarily dependent on the size of the organization (more people provide more Time resource). The productivity of the people is also a factor.

Personal Energy:

In addition to Time each person/department has some Personal Energy available as a resource. Some tasks require Personal Energy while for others it may be optional. Personal Energy can be converted into Time on the RESOURCES AVARABLE card. (see Figure 2, p 5)

The amount of Personal Energy available in a department, like the Time resource, is largely dependent on the size of the organization. The quality of work life in the organization is another factor determining the amount of Personal Energy. If, for example, the level of Vision were to fall below some critical value, or if there was insufficient Coaching of employees, the amount of Personal Energy available would be reduced. 
Information (see also "Get Info" on page 12)

At the beginning of each quarter managers have only a few of the "bits" of information they need to complete tasks. A major activity of managers in the Alpha company is to get the information necessary to accomplish the tasks they select for their departments.

In the Alpha company, managers need 3 types of information in order to complete Current Tasks. These are named according to how they are acquired:

People information that is obtained through "personal networking"

Culture information that is more easily obtained by having an "awareness of the company culture", and

Rules information that requires knowledge of the "rules \& procedures" of the organization.

Resources which are unused at the end of a quarter are carried over to the Resources Available for the following quarter.

\section{Sequence of Play...}

\section{a. ...between quarters}

Play is in rounds which can be thought of as quarters of a year. During a quarter, each manager works on his Macintosh to make the decisions for his department to earn task completion points. Managers move back and forth between cards until they have finished their allocation of resources. They then move to the next quarter where they receive additional resources and new tasks to accomplish.

Because there is data to be passed between departments at the beginning of each quarter, it is necessary for all of the managers on a team to finish their decisions for a given quarter before any of them can begin the following quarter. The game software manages this by waiting for the data of the other departments before allowing a manger to begin the next quarter.

\section{b. ...Card layout and movement}

The RESOURCES AVAILABLE card is shown in figure 2 which shows the general layout on each of the cards in WADA. Every card shows its name at the top of the card and there is also a DISCUSSION button which describes the working of that card. Buttons that take the player to each of the 9 major cards are shown at the bottom of the card. The function unique to each card is in the middle of the card. (The functions of the RESOURCES AVAILABLE card include; the display of resources, the function to allow conversion of Personal Energy into Time resources and the indicator of the current quarter of play). 


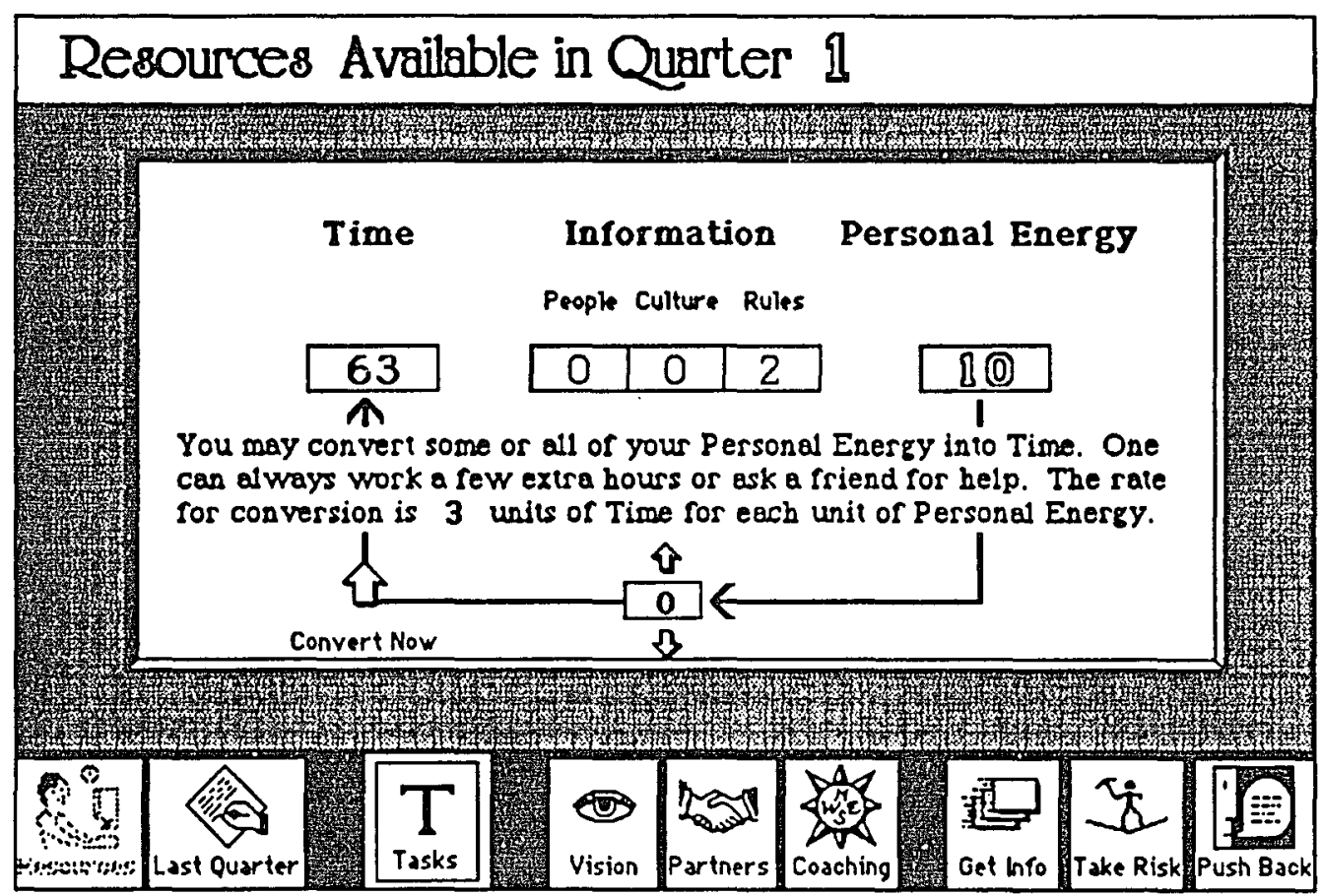

Figure 2. The Resources Available Card.

\section{c. $\quad$...within quarters}

While each manager selects which cards to visit and the order to visit them, the organization of the game implies a general pattern (indicated by the arrangement of buttons left to right across the bottom of the cards).

A. Managers begin each quarter at the RESOURCES AVAILABLE card. They usually then visit the LAST QUARTER card to get messages and results from the previous quarter. (Except for the occasional use of the conversion function, players make no decisions on these cards.)

B. Managers next visit the TASKS card to examine opportunities to earn task completion points this quarter. This card is where the majority of resource decisions are made. (See figure 3, p 7.) After selecting Current Tasks (discussed in next section) the department's information requirements are known.

C. Players then move to the cards of the development tasks (VISION, PARTNERS, COACHING) where they assign the resource amounts for these tasks. (These assignments then appear on the TASKS card.)

They also visit the remaining group of cards (GET INFO, TAKE RISKS, PUSH BACK) to consider these options. Players may return to the TASKS card to check their resource balance several times before being satisfied with their resource decisions.

D. Players advance to the next quarter from the TASKS card. 
d. Two Task Types...

There is a distinction between two general classes of tasks in "Winning at Design Automation". Both are displayed on the TASKS card.

Current Tasks earn players "task completion points". The Current Tasks that a manager may select from in a given quarter are listed on the top part of the TASKS card. Next to each task there is a specific cost for the completion of that task. (See figure 3.) Tasks that remain selected at the end of a quarter cause the associated resources to be used and points to be awarded during the update between quarters.

Current Tasks are one time opportunities to earn points. A new set of Current Tasks is present at the start of the next quarter. These tasks are independent of one another.

Players make provisional resource allocations to Current Tasks by clicking the corresponding button for each task to "on" (dark center). All of the resource allocations made on the TASKS card are reversible until you move to the next quarter. You may move to other cards and then return to change your selection of Current Tasks on the TASKS card. To change your provisional resouice allocation, simply click the button again and it will toggle to "off". (In contrast resource decisions which are made on the GET INFO, TAKE RISKS, and PUSH BACK cards are irreversible.)

Development Tasks (VISION, PARTNERS, COACHING) do not earn task completion points. Instead, the resources allocated to these tasks should be thought of as an investment in the people of the company that can have a "payoff" in the future by the way in which they change the internal conditions of the company.

Unlike the Current Tasks, the Development Tasks have continuity through time. Each is modeled as what engineers refer to as a 'level' variable. A physical analogy is the amount of gas in the tank of a car. If you add to it -- here investing resources -- the level is higher. If you drive around -- here the passage of time -- the level declines. Graphs which indicate the levels of these variables are available on each Development Task card. There are feedbacks among the Development Tasks. These are discussed in section III below and in the descriptions of the Alpha and Beta cultures.

The resource allocations for Development Tasks are also displayed on the TASKS card. Unlike Current Tasks, players set the amount of resource to spend on particular development tasks each quarter. That amount is set on the respective cards where other information is available. These resource settings are reversible until players move to the next quarter. 


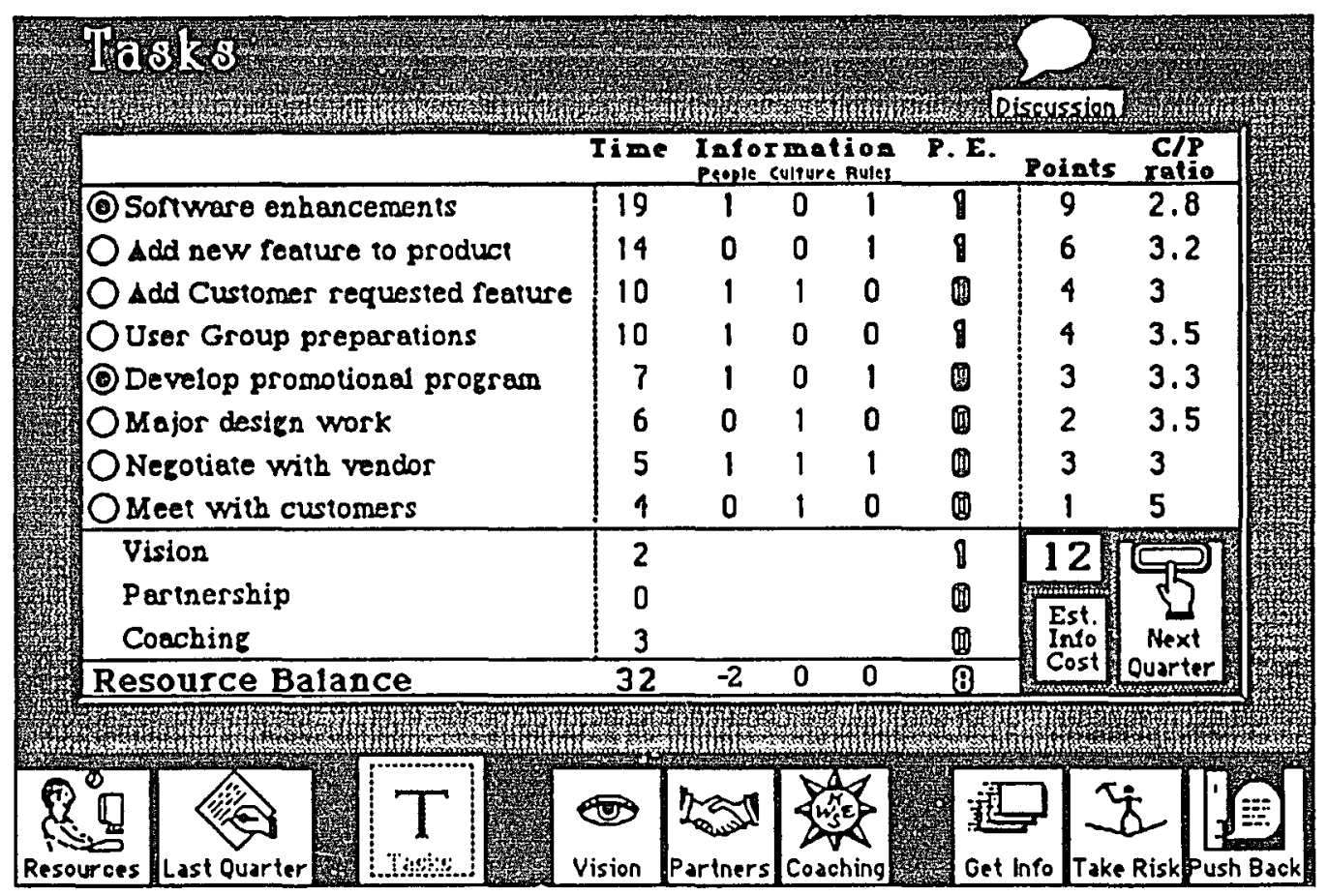

Figure 3. The TASKS card.

Discussion of figure 3. Two Current Tasks have been selected for a total of 12 points. Note the information balance for "People" information has become negative with these selections. This must be corrected -- either from the GET INFO card or by selecting Tasks with lower information requirements -- before moving to the next quarter of play.

The right most column ( $\mathrm{C} / \mathrm{P}$ ratio) presents an estimate of the resource cost of each Current Task divided by the number of points for that task. Each Time unit is given a weight of 1 as is each unit of information. Person Energy units are given the weight of 3 (the conversion ratio). This provides one obvious decision rule for players -- select those tasks with the lowest ratio. Often players are trying to conserve one type of resource and so this rule is frequently violated. Additionally, players may find that the assignment of ' 1 ' for each unit of information may not represent their success rate on the Get Info card.

\section{Resource Displays}

There are three cards on which resource information is displayed and two different displays. How player actions influence these displays is summarized here.

"Resources Available" are displayed on the RESOURCES card. (See figure 2)

The "Resource Balance" is displayed near the bottom of the TASKS card and is replicated on the GET INFO card. 
When no tasks are selected on the TASKS card, the "Resource Balance" near the bottom of that card will be exactly those showing on the RESOURCES AVAILABLE card. A display of the same information is found on the GET INFO card. As tasks are selected on the TASKS card, the "Resource Balance" is updated by subtracting the resources of all selected tasks from the "Resources Available" on the RESOURCES AVAILABLE card. This allows players to see the information they need and the remaining resources when various combinations of tasks have been selected.

Decisions on the TAKE RISKS, GET INFO, and PUSH BACK cards are immediate and irreversible. As managers commit resources from these cards, resource adjustments are made to the "Resources Available" display on the RESOURCES AVAILABLE card. Those decisions then 'trickle down' to the Resource Balance on the TASKS and GET INFO cards.

Said another way, resource actions which are NOT reversible (those on the TAKE RISKS, GET INFO, and PUSH BACK cards) are subtracted from the Available Resources.

Reversible actions (Current and Development Tasks) are not subtracted from Available Resources but instead are used to calculate the Resource Balance.

\section{Resource Display}

\begin{tabular}{l|l|l|}
\multicolumn{1}{c|}{ Resources Available } & \multicolumn{1}{c|}{ Resource Balance } \\
\cline { 2 - 3 } $\begin{array}{l}\text { where } \\
\text { displayed }\end{array}$ & \multicolumn{1}{|c|}{ Resources card } & \multicolumn{1}{c|}{$\begin{array}{l}\text { Tasks card } \\
\text { Get Info card }\end{array}$} \\
\cline { 2 - 3 } & $\begin{array}{l}\text { Indicates the resources available } \\
\text { at the start of a quarter, less those } \\
\text { resources spent ( from Take Risk, } \\
\text { Get Info or Push Back cards ) }\end{array}$ & $\begin{array}{l}\text { Indicates resources remaining } \\
\text { when selections on the Tasks } \\
\text { card are subtracted from the } \\
\text { Resources Available }\end{array}$ \\
$\begin{array}{l}\text { player } \\
\text { actions } \\
\text { affecting } \\
\text { this } \\
\text { display }\end{array}$ & $\begin{array}{l}\text { All decisions on the Take Risk, } \\
\text { Get Info or Push Back cards; } \\
\text { Moving to the next quarter }\end{array}$ & $\begin{array}{l}\text { Selection of Current Tasks on the } \\
\text { Tasks card and assignments from } \\
\text { the Development Tasks cards. } \\
\text { ( decisions on the Take Risk, } \\
\text { Get Info or Push Back cards flow } \\
\text { through from the Resources } \\
\text { Available display ) }\end{array}$ \\
\hline
\end{tabular}

Figure 4. Resource Displays 


\section{Description of specific Cards:}

\section{The Development Tasks:}

These tasks are grouped because they share several features:

1) The effect of resources allocated to th: zse tasks occurs over a long time frame. As in real life, this makes it difficult to determine the impact. Players get some understanding of the effect of the Development Tasks from the Culture descriptions and can also observe the behavior on the graphs to refine their understanding. Players do develop an appreciation of the impact of the development tasks over the 8 quarters that thicy visit the Alpha and Beta cultures.

2) They earn no task completion points and managers have flexibility is setting the amount of resources for each Development Task. This is in contrast to Current Tasks which do eam points and where the resources required for each Current Task are fixed by the simulation.

3) There is a gradual decline in each indicator, (i.e., the level of vision) through time. This implies a need to continue to invest resources to maintain the existing level.

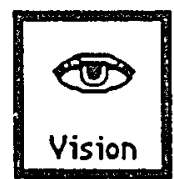

The vision in your company can be seen as composed of layers. On a regular basis, the senior managers provide statements of the vision for the entire company. Within that broad vision statement, each department can develop its own vision which is more detailed and focused (a second layer).

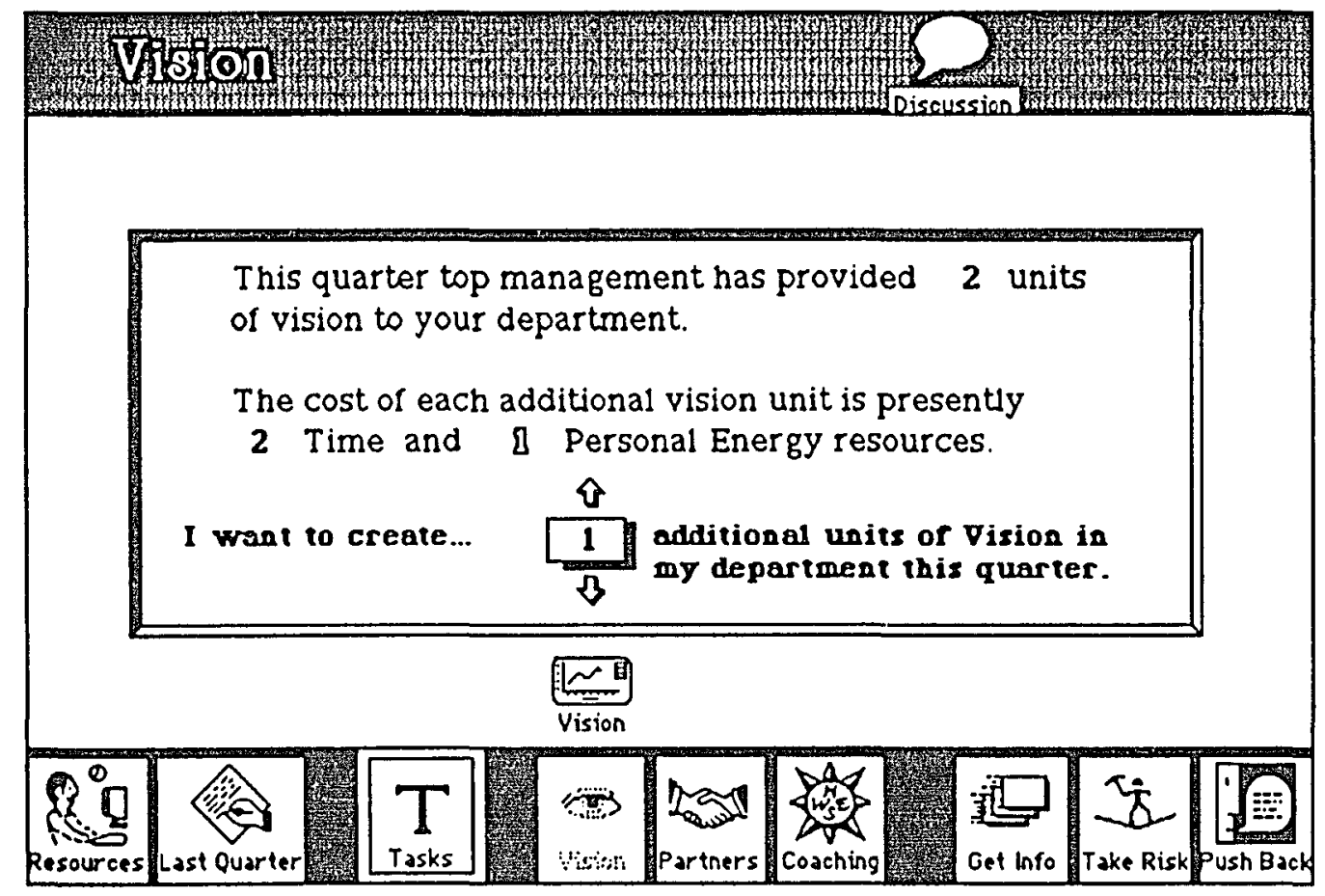

Figure 5. The Vision card. 
The vision of the company is very important to its being a top achiever. If the level of vision falls below critical levels, employees feel less connected to what is going on in the company. They are less able to see how their tasks impact the success of the company and their motivation level may decline. Without an awareness of the vision, the company feels more bureaucratic to employees.

Under ordinary conditions, the level of vision gradually declines through time as people and tasks tumover and as the external environment of the company changes and different challenges arise. To maintain the vision level, additional resources must be expended to rebuild and renew it. The level of vision for a department can be reduced suddenly when there is major change that requires updating or revising the vision such as in a major acquisition, a change in the technology which effects product positioning, or when new strategic alliances are formed.

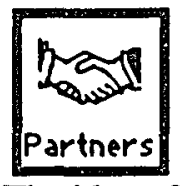

The idea of a Partnership stems from the early days of the Alpha company. The founders believed that it was important to play to win in the long term. They put extra effort into learning about the needs of individual customers so that in the future, new product features would fit those needs. This took a larger investment up front than was necessary to "close the sale", but they wanted a relationship where the customers would also invest time to improve the Alpha product. They called this long term, "win-win" relationship "Partnership". The founders of Alpha carried this same idea of Partnership to the relationship they wanted to develop with their employees.

This game addresses little in the arena of external customer relationships and so here the Partnership concept is only concerned with relationships between the departments on a team. Similar to Pushing Back (discussed below), the resources you allocate to building Partnerships must be directed at a specific department. As Partnerships are built in the real world, each side becomes more aware of the vision of the other Partner. Thus in WADA, if the level of vision in either department is too low, the further building of that Partnership can be inhibited and additional resources allocated to building that relationship would have a diminished effect.

The advantage in having a Partnership relation with the other departments is better communication and improved chances for success in Pushing Back. The long term nature of Partnerships implies a gradual building and decay relative to other aspects of the game. 


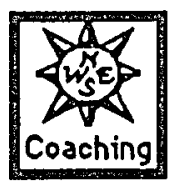

Coaching your employees can lead to improved performance. Employees who receive appropriate amounts of coaching are more productive and this can have the effect of increasing the Time and Personal Energy available in a department.

Coaching includes several activities. Part of coaching involves conveying to the coached individual the Vision of the future and showing them how their tasks contribute to the accomplishment of that vision. If the level of vision is very low, the effectiveness of resources spent coaching may be reduced. Other aspects of coaching include being a role model and removing obstacles that impede an employee's progress. The limited opportunities for coaching in each quarter apply to these coaching activities.

In WADA, coaching includes giving employees rewards for their achievements and telling stories about past achievements as a separate function. Together giving rewards and story telling are labeled "positive feedback" and this is discussed in more detail in the descriptions of the Alpha and Beta cultures. The limits on coaching resources do not apply to the giving of positive feedback. The only limit is that of available resources.

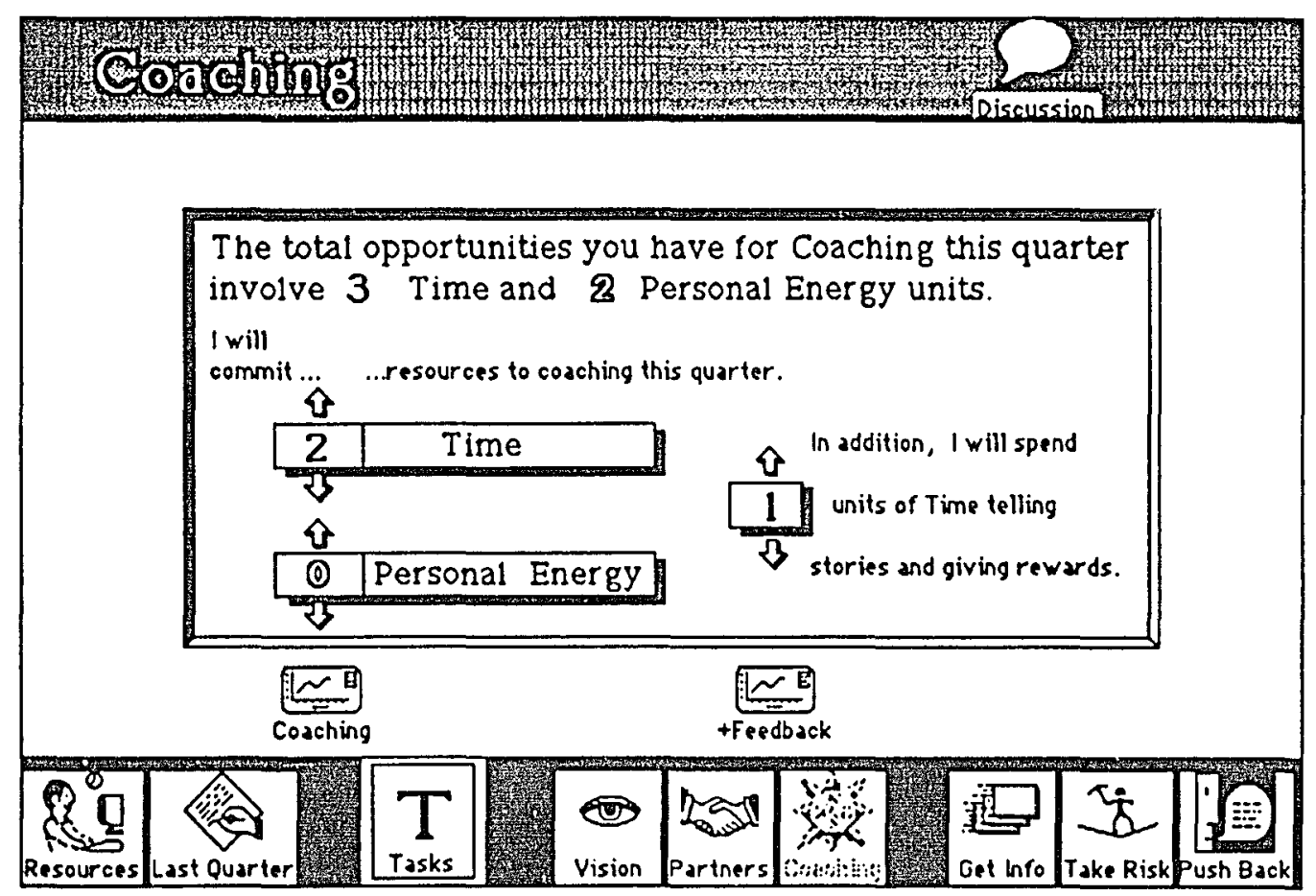

Figure 6. The Coaching card. 


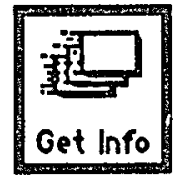

(See also Information under Resources on page 4).

There is a different approach to acquiring each of the 3 types of information needed to complete tasks. These are described in detail on the cards for the specific information types. Those cards also display the present cost of each type of information. The general pattern is for players to spend Time and provide some input such as answering a question or identification of an image. For example, players are asked questions about the culture (Alpha or Beta) when they seek culture information. (see figure 7) Correct answers yield information at a lower cost than incorrect answers.

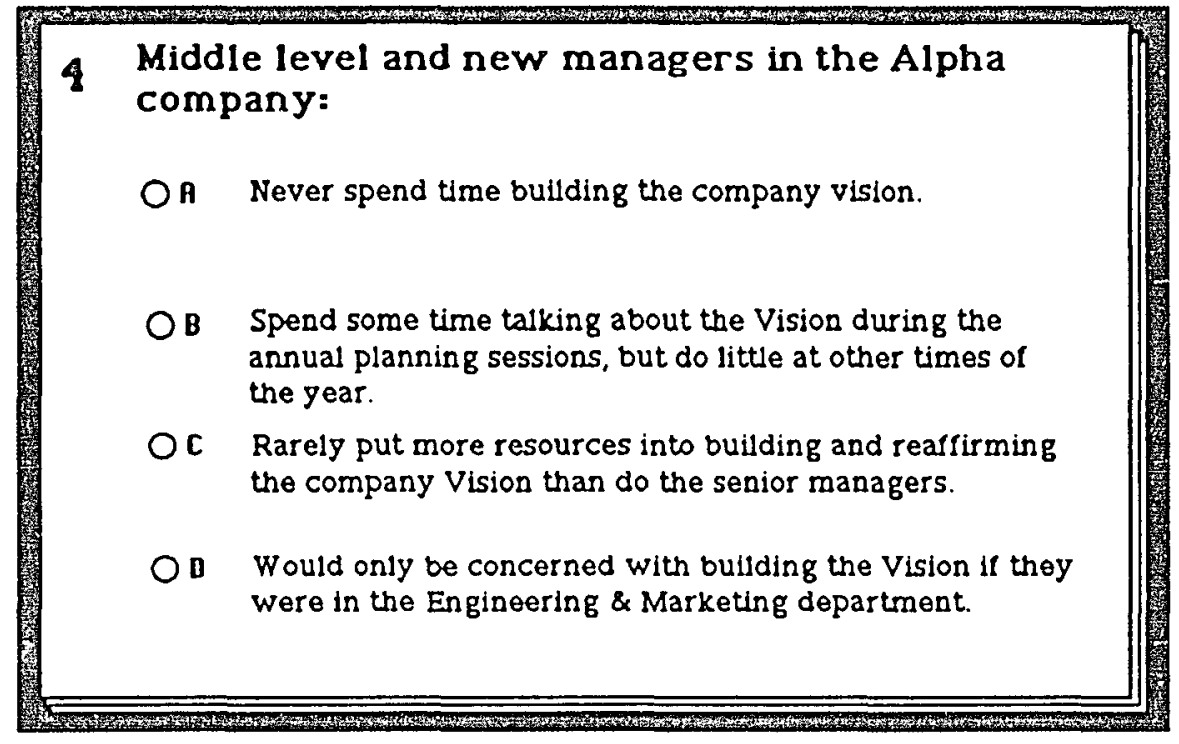

Figure 7. Example question in Get Info

The cost of getting information can change through time as various parameters of the simulation change. The cost does NOT change during a single quarter of play.

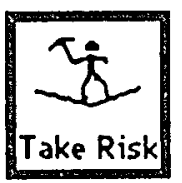

Opportunities to take risks can occur frequently in a firm with rapid growth. Commitment of resources to a risk opportunity is irreversible and immediate (when you click a button). Players usually find out their success or failure in taking a risk at the start of the next quarter.

Both the probability of success and the payoff associated with a risk opportunity are unknown and vary through time and from one specific risk opportunity to another. One factor that effects the probability of success is the level of vision in the department. If the vision falls below some minimal value, the ability of the managers to foresee 
interdependencies is reduced. This could lead them to invest resources in some risk opportunity in which the likelihood of success is very low. As the strength of the vision in a department grows the awareness of the manager is increased and, on average, the chances of success are improved. Beyond some optimal level of vision, there is no change in the likelihood of success and committing further resources to building the vision has no effect on risk taking payoffs.<smiles>Brc1ccccc1</smiles>

Pushing back involves going out of one's way to state an opinion (and when appropriate, to continue to fight for a position against opposition); to question a rule or process with the intent of finding a better way to do something; or to work to change things in the organization. Pushing back must be directed at what it is you want to change. (Otherwise it would simply be "complaining" which is of no value in either the Alpha or Beta company.)

There are a limited number of opportunities to Push Back each quarter and in the game this is represented by a limit on the total resources you can commit to Pushing Back. You direct those resources at one or more of the other departments in your company, the idea being that you are Pushing Back on something regarding that department.

Like taking risks, the success of Pushing Back involves chance. If you invest resources in Pushing Back, there may be no payoff (your efforts may be ignored or inappropriate). If there is a payoff, that benefit goes to the department at whom the Pushing Back was directed. The manager who invests in the Pushing Back receives no benefit. Any benefit from Pushing Back would appear in the next quarter.
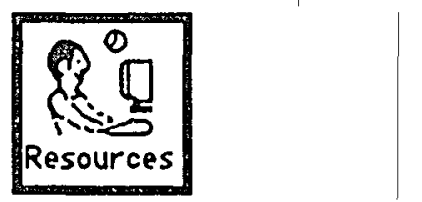

The RESOURCES card displays the resources a manager has at the start of each quarter. It is updated by any irreversible decisions during the quarter made on the GET INFO, TAKE RISK or PUSH BACK cards. It also contains a function to convert Personal Energy resources into Time. (See figure 2.)

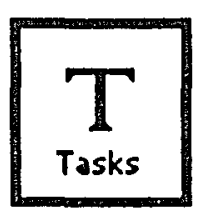

Figure 3 shows the: TASKS card and the distinction between Current Tasks and Development Tasks was discussed above. The TASKS card is the center for resource expenditures. As in the real world, there is never enough time to do all of the tasks. Here players set resource expenditures which are finalized when they move to the next quarter. The Resource Balance indicator near the bottom of the card displays the information requirements associated with various combinations of tasks. The sof tware will not allow a 
player to advance to the next quarter if this indicator has a negative balance for any of the resources.

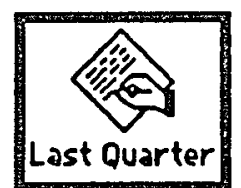

The LAST QUARTER card presents messages about various aspects of the simulation and the performance of the departments. Clicking on the message window expands it to make it easier to read long messages. Clicking again toggles the window to its initial size.

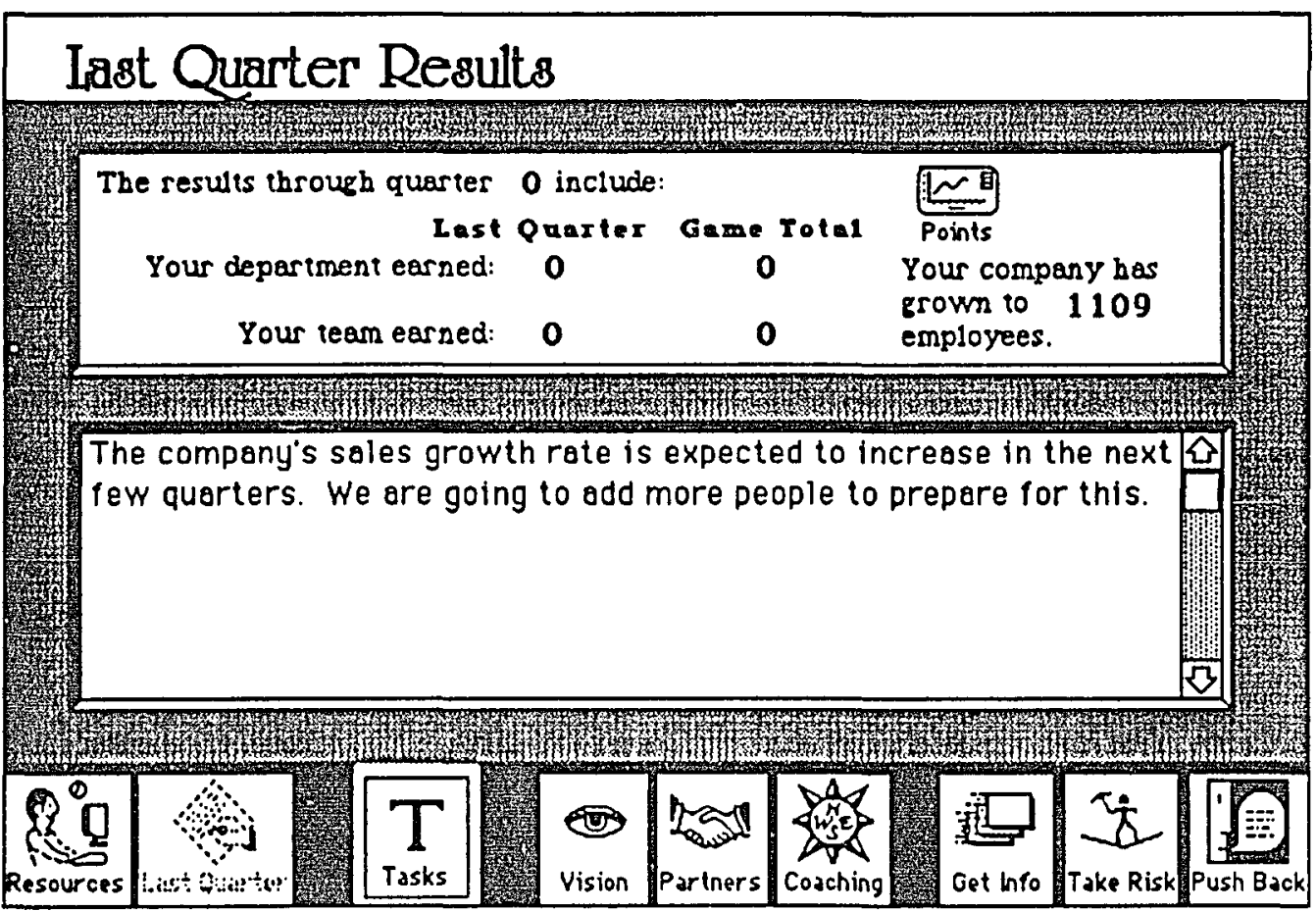

Figure 8. The Last Quarter card.

\section{Note on HyperCard ${ }^{T M}$ Environment:}

"Winning at Design Automation" (WADA) runs in the HyperCard ${ }^{\mathrm{TM}}$ programing environment. HyperCard ${ }^{\mathrm{TM}}$ is structured around the idea of a collection of electronic card images or "Cards". Each screen image the user sees on the Macintosh ${ }^{\mathrm{TM}}$ is a different card. The WADA game has about 25 cards which players may visit during the course of the game. The player navigates from card to card by clicking on "buttons", icons than are named to indicate the card to which they move the player.

Players move among the various WADA cards as they collect information and make decisions. Players may choose to visit a particular card many times. Other cards they may 
choose not to visit. Each card addresses a specific function. Section three of these rules is a description of each major card in WADA.

As the simulation software begins to execute, there is a short sequence of instruction cards that spell out what information players need about buttons, data input and related HyperCard ${ }^{\mathrm{TM}}$ issues. All player inputs are made using the mouse. There is no keyboard input required and none of the printouts or player calculations often found in business simulations. The software manages the bookkeeping effort and frees players to focus on facets of culture and rapid growth in an organization.

\section{Note on data used in the simulation:}

WADA is designed to allow facilitators to customize it for their particular organization. Examples include the choices of which "Information" tasks to incorporate and what data will be used. Also the text descriptions of "Current Tasks", "Risks", etc., can be modified by the facilitator. Players should be aware that these text descriptions are carried as labels to provide more realism but they are independent of the model that produces behavior in the simulation. Selecting one task over another should be made on the basis of; resource costs, points awarded, probability of success (if known) and other attributes that relate to the simulation model rather than the label associated with the tasks.

The simulation facilitators may provide you with goals or constraints they wish players to adhere to during the simulation that are in addition to those in these rules.

HyperCard and Macintosh are trademarks of the Apple Computer Company. 
APPENDIX F

\section{FACILITATOR'S GUIDEBOOK}




\section{"Welcome to the Class"}

We want you to experience several different cultures of high tech firms and we will discuss these and how these are similar to and different from our Mentor Graphics Culture. We have wanted to offer a class to generate some discussion of our culture for awhile. We found a game which addresses a number of cultural issues in a rapidly growing high tech firm. Experiencing the cultures of these other companies will provide a useful perspective for us to think about our MG culture. And this "trying on" different cultures is in keeping with one value of our MG culture, a tolerance of diversity.

As described in the rules, you are asked to play a "role" which is described as the "Alpha Culture". We will all be playing the same role as we learn what it is like to work in the Alpha company. We want each of you to imagine that you have left MG and are on a temporary assignment (for the next hour) in the Alpha company. You want to fit in and do as well as you can there and so learning the Alpha culture is important. This is an important part of the experience we want you to have today. We think you will best be able to perceive the similarities and differences between the Alpha culture and our MG culture if you really pretend to be an "Alphan".

When I ask people about our culture they say "I don't know how to describe it." Playing these roles will give us a model we can talk about.

It is also to your advantage while playing the simulation to know the description of the Alpha culture. Previous players have said "I wish I had studied the Alpha description better.", once they get into the game.

After you have played the game for 8 quarters, then we are going to stop and discuss how you perceived being in the Alpha culture. There is space to make notes to yourself in your rules booklet and I encourage you to make notes about anything that may be useful in our discussion after we visit the Alpha culture.

\section{Directions to plavers before Alpha Play (Players at the round tables)}

Have each person introduce themselves.

\section{Form teams.}

(With an odd number of players:

-..- play yourself or ask the technical support person (Bill) to make a foursome.

-... have 2 people play on one MAC (5 on that team)

.... ask a person to return the next day BUT

(watch the changing groups, person should return to same group)

Make-up a team name.

Decide which person will play which department.

Put team names on one flip chart (to list points each quarter)

Have Individuals read the description of the Alpha culture.

PASS OUT ALPHA HATS WHILE THEY READ CULTURE DESCRIPTION. 
Tell them they must leave culture descriptions on round tables (They may not take them to their MACs)

They should make notes of similarities / differences with MG culture in back of book

(While they read, record which person is at which machine)

Have each Team discuss the Alpha culture.

They should summarize the culture on their flip chart. Each team then presents their summary to the entire group.

(3-4 minutes for each team)

\section{HAVE PLAYERS PUT ON ALPHA HATS IF THEY HAVE NOT}

\section{SUGGEST TO PLAYERS}

THAT THEY STOP FOR A MOMENT \& IMAGINE being in THE ALPHA CULTURE (See directions on last page of Alpha description) "close your eyes and imagine you are visiting Alpha \& you want to fit into this culture during your short visit."

\section{Walk through first quarter of Play. Let them make decisions on} their Macs while following vou on overhead.

LIST OF "DON'TS" (on flip chart) DON'T TURN OFF MACs !!

DON'T MOVE MAC (previously one MAC became unplugged)

Don't Touch keyboard.

Walk them through the 1st auarter on the overhead.

Ask them to follow you and make decisions WHEN YOU ASK THEM. Point out that EVERYONE finds the game complex the first quarter. By the third quarter, everyone is comfortable.

Point out to them features:

show hot point of finger at tip

show expanding message box on Last Quarter card.

(ENCOURAGE THEM TO USE IT !!)

describe Last Quarter points vs total (includes risk)

show larger buttons for arrow icons (hold down option \& control keys)

show Push Back sequence (resources THEN department)

show graph buttons on Development tasks

(ENCOURAGE THEM TO USE THESE !!)

mention that there are some hints in 'discussions'

DISCUSS "immediate resource commitment" on: Get Info, Risk, Push, convert vs "allocation" on Tasks, Vision, Partners, Coaching

show each type of information and cost of information

maze -- distance calculated only when pointer is on button. pointer "hot point"

On tasks card: $\mathrm{C} / \mathrm{P}$ is estimate that is calculated before game. info costs can change.

End of Quarter update: occasional network problem, call if needed. 
After the walk through on the overhead

Express the need to keep meving to get through 8-10 quarters of play. The interactions between culture and growth they are to observe occur over several quarters. No one decision can kill you. Prod slow players.

Tell them about previous experiences:

"A problem I had:" after selecting tasks and leaving some resource to get information, I find it required much more resource to get all the needed information.

Solution: When resources go negative, select tasks lower on the list (or eliminate tasks) to reduce the information requirement.

Mention the Emphasis on playing the Alpha role. example: risk decisions: ask yourself, "what would an Alphan do?", NOT "what would I do." You can record discrepancies in the space provided at the back of rules booklet.

Suggest they record thoughts / comments in rules booklet during the between quarter update.

\section{During the Alpha plav:}

EACH Quarter, record the team points for each team on the

flipchart and call attention to these.

'Blenders' at the end of $Q 2$ and Q5 -. 5 min each.

players return to their round tables.

Suggest players help each other to figure this out.

Ask how the Alpha culture is/is not like MG.

Have them write comments in back of rules book.

\section{Keep teams moving}

Give slow teams shorter blenders.

Indicate they won't win if they play fewer quarters.

"no one decision is critical", "observing patterns over several quarters is the key"

Play a few quarters (at least 4) on the overhead machine to have behavior to show during the Beta walk through. Play the ALPHA strategy to you can show how you slipped into bureaucracy.

\section{At the end of Alpha play}

Use expanding message window to reset game.

Players to answer multiple choice questions about Alpha before debriefing discussion.

\section{Debrief after Alpha play} (After plavers answer the multiple choice questions at the end of Q8)

What were your feelings about being at the Alpha company? 
Did you follow the Alphan role?

What parts of the culture were similar to MG? How similar?

what parts different?

How well was Alpha company doing? (Was the business successful?)

Did the Alpha company change as it grew?

How would you describe those changes?

Did your feelings about being in the company change?

How important is the vision in the Alpha company?

How important is Pushing Back in the Alpha company?

Did anyone take any risks? Were you successful?

Did anyone do any Pushing Back? Were you successful?

\section{Set up before Beta:}

Hide Alpha list of teams \& scores.

COLLECT ALPHA HATS

COLLECT ALL ALPHA CULTURE DESCRIPTIONS

MAKE New list of teams to show Quality points.

As with Alpha (see above) GIVE OUT BETA HATS

Individuals read Beta culture ( $15 \mathrm{~min}$ ), then discuss with team members. Mark flipcharts \& discuss.

Brief discussion: How similar is Beta to MG culture (esp. video).

\section{Dinections to plavers before Beta Plav}

The Beta culture is more difficult to play as the change in rules gives you two simultaneous goals. A tendency for Beta to take longer so keep moving.

Don't fall behind in quality. It is difficult to recover. With rapid growth it is easy to misjudge the needed increase in development tasks for the next quarter. Note that the Beta company just had a major acquisition and this has diluted their development task strategy -- see the graph history.

Also I noticed that it required an adjustment in my thinking to the "smaller" company (the same size you started with Alpha). In Q8 you may have earned 40 points. Now in Q1 10 points seems very small, but it may not have been much different than what you did in Alpha Q1.

People have said they use the graphs more in the Beta game. This is one key to success in keeping the small company atmosphere. Also they tend to set the development tasks first, then do current tasks with the remaining resources. Many did the opposite when playing Alpha. The Setting of the development tasks is more precise in the Beta culture.

The Beta rule change may suggest two strategies,

1) play Beta correctly and try to keep the small company atmosphere, $O R, 2)$ take the penalty \& go for the maximum points.

Because of the class goal of seeing others cultures, I hope you will chose \#1. 
It is possible that with skillful play you can match your Alpha score without losing the small company atmosphere in Beta. I don't think you will all do that but I would expect at least one of you to do so.

Walk through notes:

show the quality graph and Alpha history

show several example development task graphs (vision, positive feedback) and show Alpha history. note difference between Alpha \& Beta cultural viewpoints as shown in graphs.

note that the Beta view is not part of the Alpha culture \& Alphans never see these perspectives.

\section{Debrief after Beta}

How did it feel to be a Betan?

Did you follow the Betan Role? (Was it difficult?)

What similarities / differences with MG?

How well was the Beta company doing (in a business sense)?

In which company would you prefer to work?

In which company did you make the most points? Why?

In which company would you prefer to work?

Which company is closer to MG? (today?) (in the company ideals?)

As you play Alpha many of us don't try the Beta strategy because we have cultural blinders. The Aphans don't SEE the possibility of Beta success. How can we avoid this in our life? Practice one of our primary culture values: Push Back on our ideas, systems, our beliefs \& assumptions and check them. This underscores the need to take risks and try new approaches to see if they will work.

What steps can YQU take to keep the small company atmosphere at Mentor Graphics. (Write in comments section of rules then discuss)

NOTE ON DISCUSSION WITH OTHERS AFTER TODAYS SESSION

COLLECT ALL HATS

COLLECT ALL RULES BOOKLETS

COLLECT ALL CULTURE DESCRIPTIONS

I am asking other managers to play this simulation. I am certain that some of you will have the opportunity to talk to them about it. The two cultures in the game were designed to show some contrasts and I think it is important that one plays both cultures to get the full benefit the game experience can offer. So I ask that in any conversations you have with friends BEFORE that person plays the game, that you refrain from telling them any of the details about Alpha and Beta. 


\section{APPENDIX G}

WADA SIMULATION MODEL 
Figure 59 displays the basic model that drives the WINNING AT DESIGN AUTOMATION simulation. Growth in the size of the simulated company, indicated by "number of employees" to participants, is driven by the passage of time. As size increases so does the amount of time resource available to do tasks (because more employees contribute more time). Size determines the X-axis of the Quality Graph (Figures 17 and 28).

\section{The "Quality Growth" Choice}

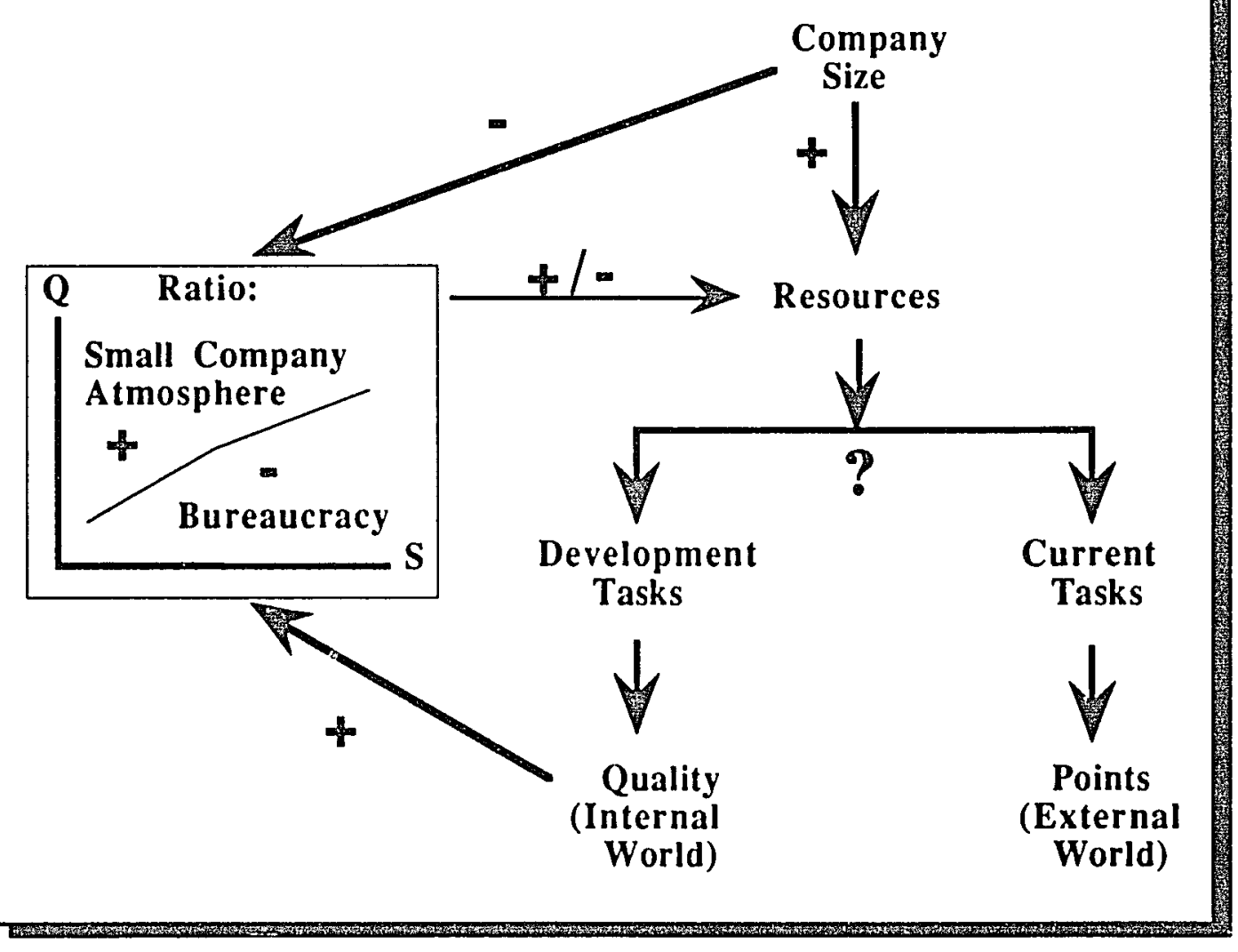

Figure 59. The basic model of the WADA simulation.

The basic choice participants face is the allocation their time between Current Tasks which earn the points measuring their success against other teams (and represent success on traditional business indicators, e.g., 
market share, earnings, product innovation, customer service) and the Development Tasks (e.g., Vision, Coaching, Partnerships, Rewards) which earn no points but determine the "Quality" for their department.

The quality measure determines the Y-axis on the Quality Graph. The ratio of Quality and Size determines the culture in their simulated department. When that ratio is high there is a "small company atmosphere ${ }^{n}$ and employees have considerable energy, enthusiasm and commitment that act to increase the time resources available. When the ratio is low, there is a "bureaucratic culture" and employees have little energy, enthusiasm or commitment. Bureaucratic drag acts to reduce the time resources available.

The goal for players is to find the balance between Current Task and Development Task investment that optimizes total points over the eight simulated quarters they play. In any given quarter, the optimal strategy is to invest only in Current Tasks - all resources earn points. However over a period of several quarters this is never the optimal strategy because with no investment in the Development Tasks, quality declines and reduces resources available. Because of delays in the impact of low quality, the balance participants seek represents the tension between short term and long term investment. Competitive pressures focus the organization on the short term, while a longer term perspective is optimal in WADA, consistent with the beliefs of the founders of Mentor Graphics. 